\title{
Near Vertical Incidence Skywave
}

\author{
Interaction of Antenna
}

and Propagation Mechanism

Ben A. Witvliet

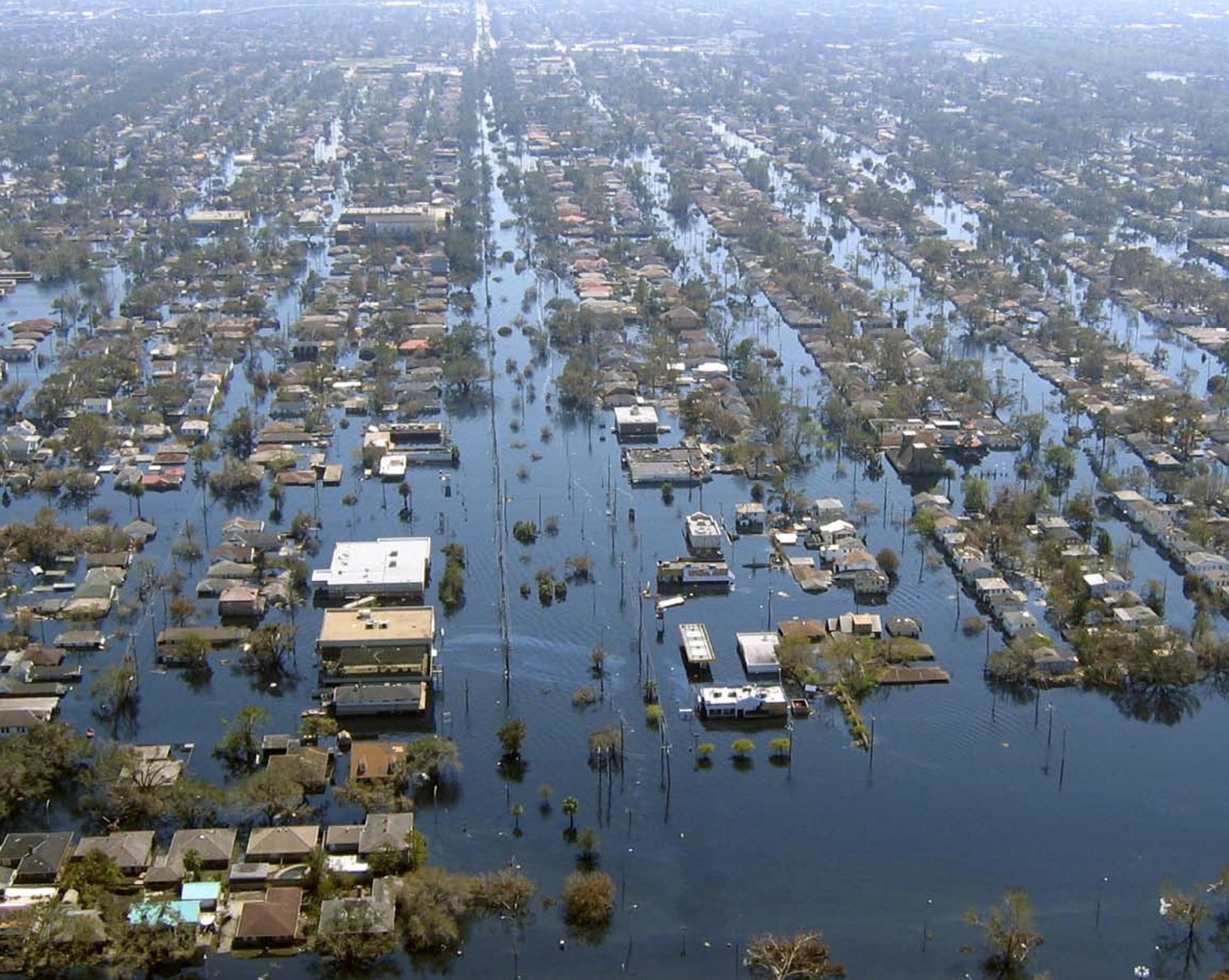




\title{
Near Vertical Incidence Skywave
}

\author{
Interaction of Antenna
}

and Propagation Mechanism

\author{
Ben A. Witvliet
}




\section{Composition of the graduation committee:}

\section{Chairman \& Secretary:}

prof. dr. P. M. G. Apers University of Twente, The Netherlands

\section{Supervisor:}

prof. dr. ir. C. H. Slump University of Twente, The Netherlands

\section{Co-supervisors:}

dr. ir. M. J. Bentum

University of Twente, The Netherlands

dr. ir. R. Schiphorst

University of Twente, The Netherlands

\section{Referee:}

dr. H. K. Leonhard

Radiocommunications Agency Netherlands

\section{Members:}

prof. dr. ir. F. B. J. Leferink prof. dr. ir. F. E. van Vliet prof. dr. ir. A. B. Smolders dr. ing. I. E. Lager
University of Twente, The Netherlands University of Twente, The Netherlands Eindhoven University of Technology, The Netherlands Delft University of Technology, The Netherlands

The research presented in this thesis was carried out at the Telecommunication Engineering group and the Signals and Systems group, Faculty of Electrical Engineering, Mathematics and Computer Science, University of Twente, P. O. Box 217, 7500 AE Enschede, the Netherlands.

ISBN: $978-90-365-3938-8$

DOI: $\quad 10.3990 / 1.9789036539388$

URL: http://dx.doi.org/10.3990/1.9789036539388

Copyright @ 2015 by Ben A. Witvliet.

All rights reserved. No part of this publication may be reproduced by print, photocopy or any other means without the written permission of the copyright owner.

Printed by Gildeprint, Enschede, The Netherlands. 


\title{
NEAR VERTICAL INCIDENCE SKYWAVE
}

\author{
INTERACTION OF ANTENNA \\ AND PROPAGATION MECHANISM
}

\section{PROEFSCHRIFT}

ter verkrijging van

de graad van doctor aan de Universiteit Twente, op gezag van de Rector Magnificus, prof. dr. H. Brinksma, volgens besluit van het College voor Promoties in het openbaar te verdedigen op woensdag 2 december 2015 om 14:45 uur

door

Benjamin Axel Witvliet

geboren op 6 december 1961

te Biak, Nederlands Nieuw Guinea 
Dit proefschrift is goedgekeurd door:

De promotor:

prof. dr. ir. C. H. Slump

De assistent-promotoren:

dr. ir. M. J. Bentum

dr. ir. R. Schiphorst 


\section{Samenvatting}

In gebieden waar geen telecommunicatie-infrastructuur is, of wanneer die infrastructuur door een natuurramp is verwoest, kan Near Vertical Incidence Skywave (NVIS) propagatie voor een verbinding met de buitenwereld zorgen.

Om gebruik te maken van NVIS moeten de radiogolven recht omhoog worden gezonden, waar, op een hoogte tussen 80 en $350 \mathrm{~km}$, de ionosfeer deze golven terugbuigt naar de aarde. Vanwege het frequentieafhankelijke karakter van de propagatie moet bij de keuze van de werkfrequentie rekening worden gehouden met parameters van de ionosfeer. Typische werkfrequenties liggen tussen 3 en $10 \mathrm{MHz}$.

Door de grote reflectiehoogte wordt een aaneengesloten gebied van tenminste $400 \times 400$ $\mathrm{km}$ rondom de zender bestreken. Aangezien de radiogolven onder een steile hoek naar beneden komen, vindt geen afscherming plaats door grote objecten zoals gebouwen of bergruggen plaats.

Aangezien NVIS niet afhankelijk is van een netwerk of netwerkoperator is snelle uitrol mogelijk. Bovendien zijn de antennes en radioapparatuur relatief eenvoudig te maken en onderhouden, zelfs in landen met een lager technologisch niveau. Deze aspecten maken NVIS radiocommunicatie bij uitstek geschikt voor communicatie na natuurrampen en voor onderwijs en medische zorg op afstand in arme en/of afgelegen gebieden.

Onderzoek naar de inzet van NVIS propagatie voor point-to-point verbindingen of omroep heeft verspreid over tientallen jaren plaatsgevonden en bestrijkt een groot aantal onderwerpen. In dit proefschrift worden blinde vlekken in dat onderzoeksgebied geïdentificeerd en bestudeerd, om zo bestaand onderzoek aan te vullen en te verbinden. Daarbij ligt de focus op antennes en propagatie.

De volgende onderzoeksvragen werden geformuleerd:

1. Hoe functioneert het NVIS propagatiemechanisme, en welke parameters van dit mechanisme zijn van belang voor de optimalisatie van NVIS telecommunicatiesystemen?

2. Hoe kunnen we de NVIS antenne optimaliseren zodat (a) het sterkste signaal wordt geproduceerd in het verzorgingsgebied, en (b) zodat de grootste signaal-ruisverhouding wordt gerealiseerd bij ontvangst van signalen uit dat verzorgingsgebied?

3. Hoe groot is de interactie tussen NVIS antenne en NVIS propagatiemechanisme?

De nadruk van het onderzoek ligt op empirische verificatie van de effectiviteit van antennes en van propagatieverschijnselen en een aantal nieuwe meetmethoden is ontwikkeld om dit 
mogelijk te maken. De metingen zijn uitgevoerd in Nederland $\left(52^{\circ} \mathrm{N}, 6^{\circ} \mathrm{O}\right)$, en worden geacht representatief te zijn voor het gebied tussen 40 en 60 graden Noorderbreedte.

Onderzoek met betrekking tot het NVIS propagatiemechanisme laat zien dat elevatiehoek, polarisatie, fading en ruis de belangrijkste parameters zijn bij de optimalisatie van NVIS telecommunicatiesystemen. De relatie tussen elevatiehoek en afstand is bepaald als functie van de werkfrequentie en het zonnevlekkengetal, en door meting bevestigd. Door middel van metingen is aangetoond dat NVIS al vanaf korte afstanden ( $20 \mathrm{~km}$ op $7 \mathrm{MHz}$ ) dominant is ten opzichte van de grondgolf. De metingen laten ook zien dat NVIS efficiënt is: één 100 Watt zender bestrijkt een gebied van $400 \times 400 \mathrm{~km}$ met 35 tot $55 \mathrm{~dB}$ signaal-ruisverhouding. In de nachturen is propagatie waargenomen over een afstand van $110 \mathrm{~km}$, op een frequentie boven de kritische frequentie van de ionosfeer, met een fluctuerend karakter dat veel weg heeft van verstrooiing (scattering) en niet lijkt op grondgolfpropagatie.

Het belang van de propagatie van karakteristieke golven in de ionosfeer is aangetoond door middel van metingen en laat bijna perfect circulaire polarisatie van de neergaande golven zien, met een grote ( $>25 \mathrm{~dB}$ ) scheiding tussen beide karakteristieke golven. Een antenne met slechts $0,5 \times 0,5 \lambda$ footprint is ontworpen, waarmee de beide karakteristieke golven gescheiden kunnen worden ontvangen. Toepassing hiervan voor diversiteitsontvangst (diversity) resulteert in 8 tot $10 \mathrm{~dB}$ reductie van het benodigd zendvermogen.

Onderzoek laat zien dat de optimalisatie van zend- en ontvangstantenne een verschillende benadering vraagt en verschillende optima oplevert. Optimalisatie van de ontvangstantenne vergt kennis van de propagatie van elektromagnetische omgevingsruis, waarbij zowel de polarisatie als de verdeling over de ruimtehoeken van belang is. Eerste experimenten laten zien dat de verdeling over de ruimtehoeken niet uniform is. Een nieuwe methode om de effectiviteit van meetantennes voor omgevingsruis te bepalen wordt beschreven.

Voor het op locatie vergelijken van NVIS antennes is een nieuwe meetmethode ontwikkeld die gebruik maakt van NVIS propagatie. Met deze methode is de optimale hoogte van een horizontale dipool als zendantenne bepaald. Die ligt tussen 0,18 en 0,22 $\lambda$ voor de meeste grondsoorten. Het optimum van de ontvangstantenne ligt rond $0.16 \lambda$, maar die is minder kritisch. In tegenstelling tot wat vaak aangenomen wordt presteren laag opgestelde dipolen slecht: een dipoolantenne op 0,02 $\lambda$ hoogte is 11 to $12 \mathrm{~dB}$ minder effectief dan het optimum bij zenden, en 2 tot $6 \mathrm{~dB}$ minder effectief bij ontvangst. Zo'n lage dipoolantenne is echter nog altijd $12 \mathrm{~dB}$ effectiever dan een sprietantenne op een auto.

Interactie tussen de NVIS antenne en het NVIS propagatiemechanisme is aangetoond. Naar verwachting geeft optimalisatie waarbij antenne en propagatiemechanisme als een hybride systeem worden beschouwd betere resultaten dan wanneer de antenne alleen wordt geoptimaliseerd. 
- vii - 
- viii - 


\section{Summary}

In areas where no telecommunication infrastructure exists, or when that infrastructure is destroyed by a natural disaster, Near Vertical Incidence Skywave (NVIS) radio wave propagation may provide a lifeline to the outside world.

To exploit NVIS propagation, radio waves are transmitted straight up, where, at heights between 80 and $350 \mathrm{~km}$, the ionosphere will bend these waves back towards the earth. The frequency dependent character of the radiowave propagation requires that operating frequencies are chosen considering ionospheric parameters. Typical frequencies are between 3 and $10 \mathrm{MHz}$.

Due to the great reflection height a large continuous area around the transmitter, exceeding $400 \times 400 \mathrm{~km}$, will be covered. As the downward waves arrive at steep angles, large objects such as buildings and mountain ridges cannot block the NVIS radio path. The independence of a network operator enables quick deployment, and the antennas and radio equipment are relatively easily to build and maintain, even in countries with a lower technological standard. These aspects make NVIS radio communication especially suited for disaster relief operations and tele-education and tele-medicine in poor and/or remote regions.

Research into the use of NVIS propagation for point-to-point links and broadcasting is spread over several decades and encompasses a large number of subjects. In this thesis, specific blank spots in the NVIS research field are identified and targeted, to augment and connect existing research, with a focus on antennas and propagation.

The following research questions were formulated:

1. How does the NVIS propagation mechanism function, and what parameters of this mechanism are important for NVIS telecommunication system optimization?

2. How can we optimize the NVIS antenna to (a) produce the strongest signal across the coverage area, and (b) to realize the greatest signal-to-noise ratio (SNR) on reception of signals from that coverage area?

3. How important is the interaction between NVIS antenna and propagation mechanism?

Emphasis of the research is on empirical verification of antenna performance and propagation phenomena, and several novel measurement methods are introduced for this purpose. The measurements are performed in The Netherlands $\left(52^{\circ} \mathrm{N}, 6^{\circ} \mathrm{E}\right)$, and are considered representative for mid-latitudes in the Northern hemisphere.

Investigations into the NVIS propagation mechanism shows that elevation angles, polarization, fading and noise are the most important parameters to consider in NVIS 
telecommunication system optimization. The relationship between elevation angle and coverage distance is established as a function of frequency and sunspot number, and confirmed by measurement. The dominance of NVIS over groundwave propagation is shown to start at short distances (20 km at $7 \mathrm{MHz}$ ). Measurements show that NVIS propagation is efficient: one 100 Watt transmitter will cover a $400 \times 400 \mathrm{~km}$ area with 35 to $55 \mathrm{~dB}$ SNR. Nighttime propagation over $110 \mathrm{~km}$ distance is observed above the critical frequency of the ionosphere, showing signal fluctuation similar to scattering and unlike ground wave propagation.

The importance of characteristic wave propagation in NVIS has been demonstrated by measurement, showing nearly perfectly circular polarization of downward waves and high isolation $(>25 \mathrm{~dB}$ ) between both characteristic waves. An antenna with only $0.5 \times 0.5 \lambda$ footprint is designed that provides separate reception of both characteristic waves. When applied for characteristic wave diversity reception, a reduction of 8 to $11 \mathrm{~dB}$ of the necessary transmit power can be realized.

Investigations show that NVIS transmit and receive antenna optimizations require a different approach, and result in different optima. Receive antenna optimization requires knowledge of the propagation of electromagnetic ambient noise (radio noise), considering both angular distribution and polarization. Initial experiments indicate that the angular distribution is not uniform. A novel method to evaluate the performance of radio noise measurement antennas is described.

For in-situ antenna performance comparison a new method is designed using live NVIS propagation. With this method, the optimum transmit antenna height of a horizontal dipole used as transmit antenna is determined, ranging from 0.18 to $0.22 \lambda$ for most soil types. For a receive antenna this is around $0.16 \lambda$, but that optimum is less pronounced. Contrary to popular believe, low dipole antennas are poor performers: a dipole antenna at a height of $0.02 \lambda$ is 11 to $12 \mathrm{~dB}$ less effective than the optimum on transmission and 2 to $6 \mathrm{~dB}$ less effective on reception. However, such a low dipole antenna will still outperform a car whip antenna by $12 \mathrm{~dB}$.

Significant interaction between the NVIS antenna and the NVIS propagation mechanism is shown, and optimization considering antenna and propagation as a hybrid system is likely to yield better results than isolated optimization of the antenna alone. 
- xi - 
- xii - 


\section{Contents}

Samenvatting

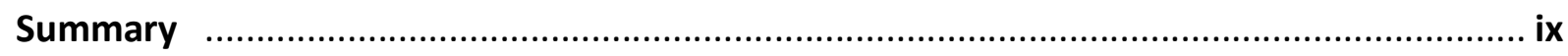

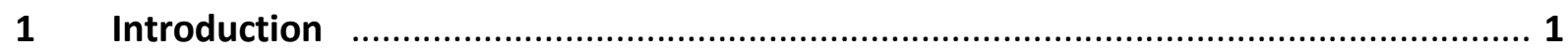

1.1 A telecommunication dependent society …................................................... 3

1.1.1 When the lines go down ............................................................... 3

1.1.2 Areas without telecommunication infrastructure ............................. 5

1.2 Near Vertical Incidence Skywave (NVIS) ....................................................... 5

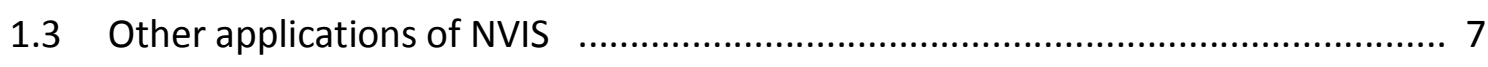

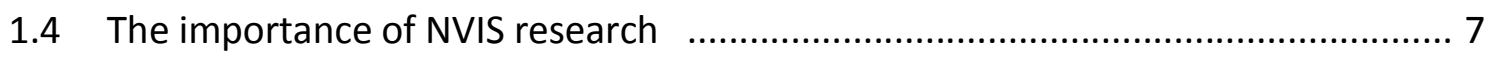

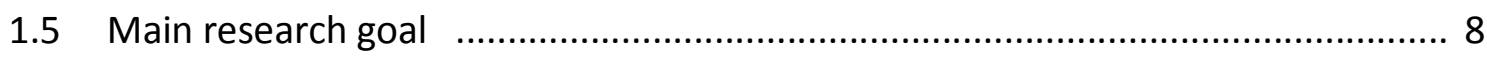

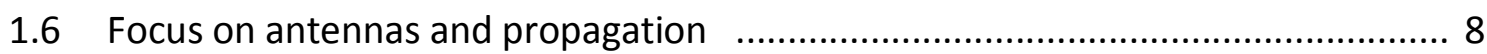

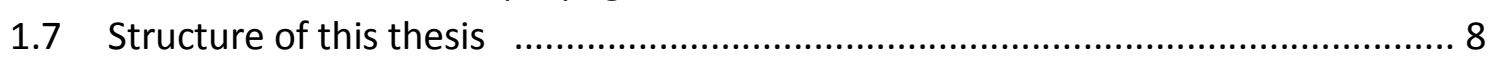

2 Communication via Near Vertical Incidence Skywave propagation: an overview ........ 11

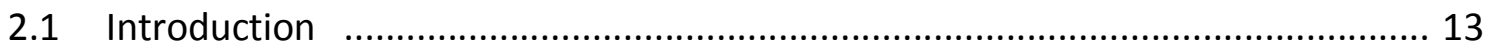

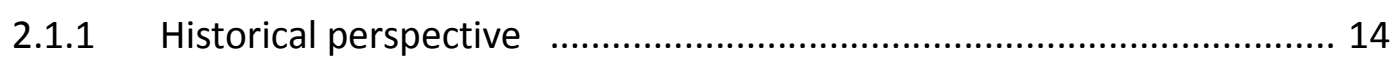

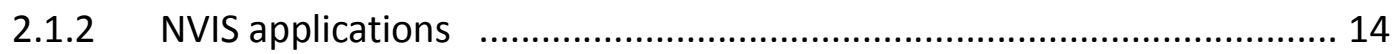

2.1.3 NVIS communication system optimization ..................................... 15

2.2 Near Vertical Incidence Skywave propagation ................................................. 16

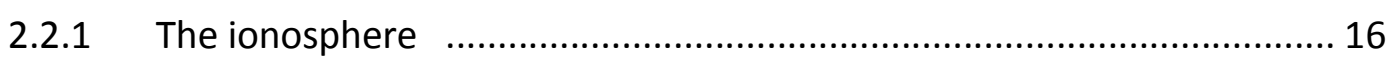

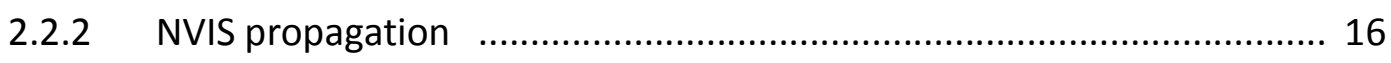

2.2.3 Characteristic wave propagation .................................................... 17

2.2.4 Diurnal variation and solar cycle ...................................................... 18

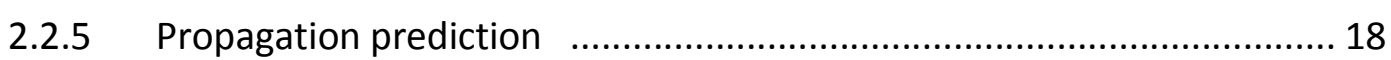

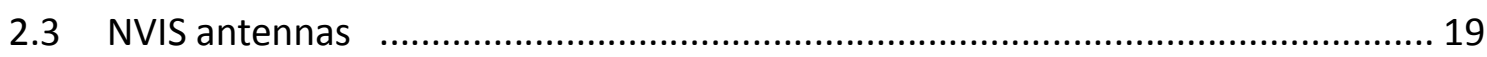

2.3.1 NVIS antennas for fixed installations .............................................. 19

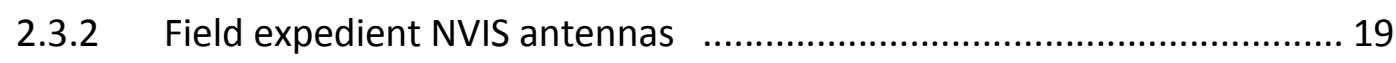

2.3.3 NVIS antennas for mobile use ......................................................... 20

2.3.4 Antennas for NVIS reception ......................................................... 21

2.3.5 In-situ NVIS antenna measurement ............................................. 21

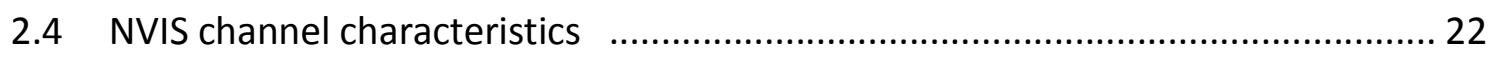

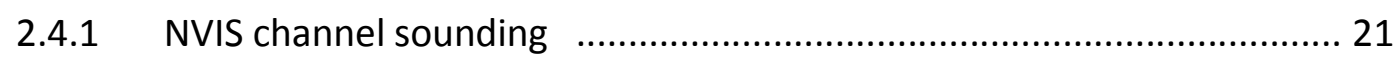

2.4.1.1 Fading ................................................................................ 22

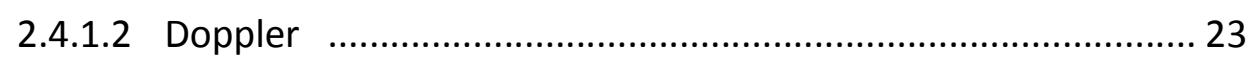

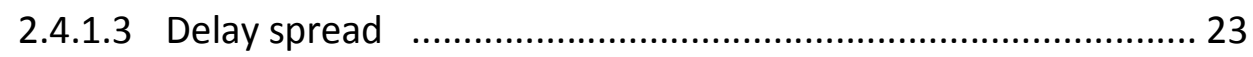

2.4.2 NVIS channel modelling ............................................................... 24 
2.4.3 NVIS channel characteristics as function of the latitude .................... 25

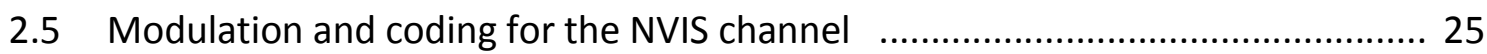

2.5.1 Data transmission protocols for NVIS communications ........................ 25

2.5.2 Experimental modulation and coding ................................................ 26

2.5.3 Diversity and MIMO ….............................................................. 27

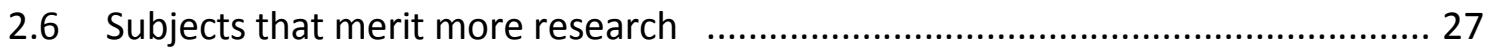

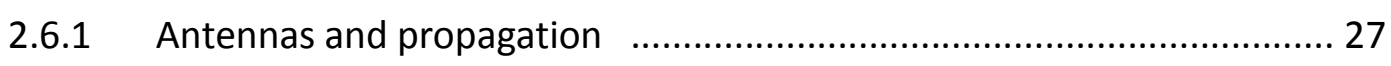

2.6.2 Channel, modulation and coding ....................................................... 28

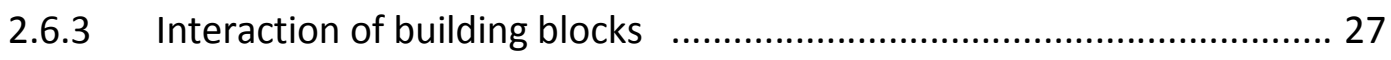

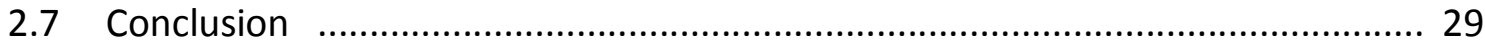

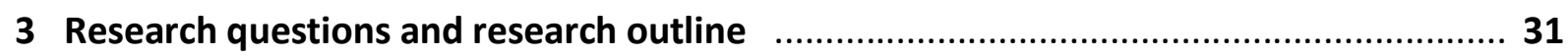

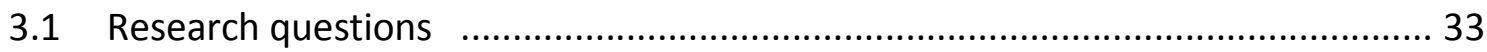

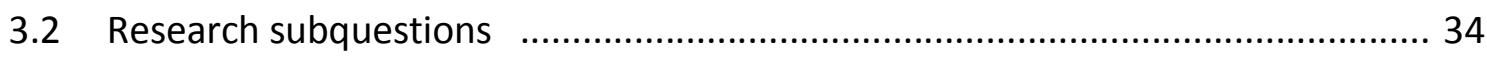

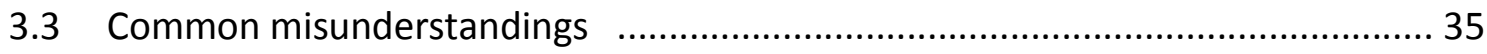

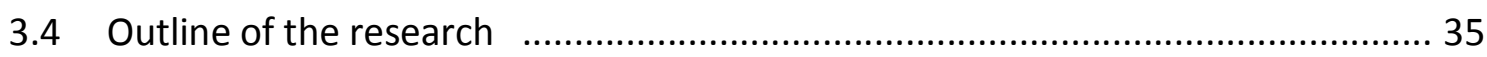

3.4.1 Elevation angle versus distance ...................................................... 35

3.4.2 Antenna performance versus height above ground ........................... 36

3.4.3 The role of radio wave polarization ................................................... 36

4 Near Vertical Incidence Skywave propagation: elevation angles and optimum antenna height for horizontal dipole antennas ....... 37

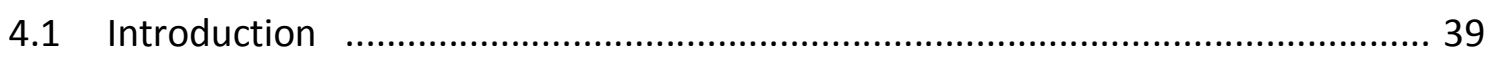

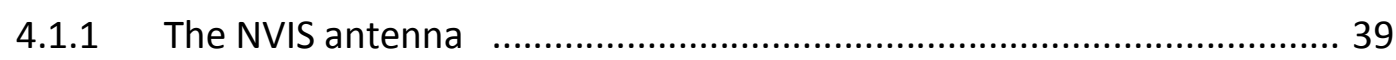

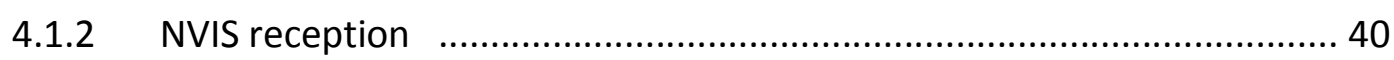

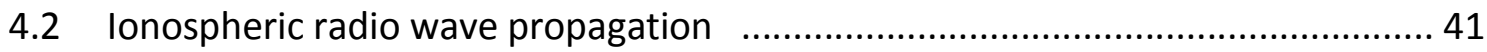

4.2.1 Frequency dependency of ionospheric propagation .......................... 43

4.2.2 Variability of ionospheric propagation ................................................ 44

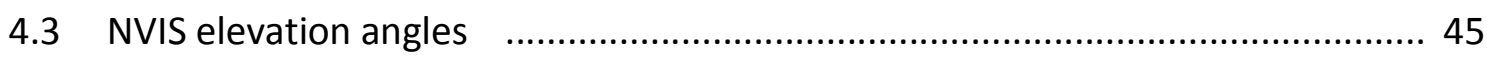

4.3.1 NVIS elevation angle simulations ................................................ 45

4.3.2 NVIS elevation angle measurements ............................................. 48

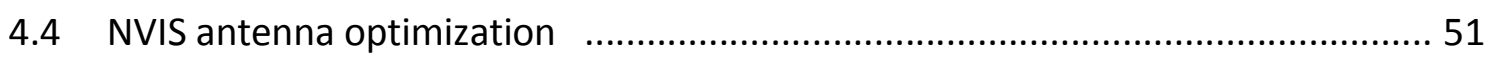

4.4.1 Influence of antenna height .......................................................... 53

4.4.2 Simulated optimum NVIS transmit antenna height ........................... 54

4.4.3 Simulated optimum NVIS receive antenna height ............................. 57

4.5 Comparison of HF antenna performance in the presence of fading .................... 59

4.5.1 Proposed new evaluation method ..................................................... 59

4.5.2 Practical realization of the proposed method ....................................... 61

4.5.3 Empirical verification of the optimum NVIS transmit antenna height .. 63

4.5.4 Empirical verification of the optimum NVIS receive antenna height ....67 67 
4.6 Analysis and discussion

4.6.1 Sensitivity to frequency and coverage area size ................................ 70

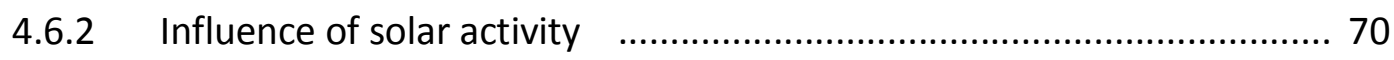

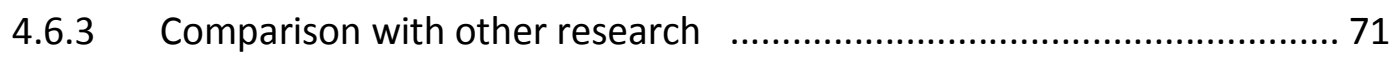

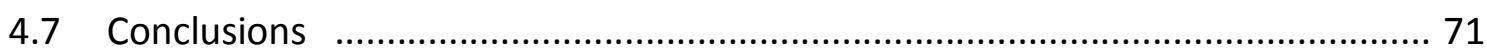

5 A novel method for the evaluation of polarization and hemisphere coverage of

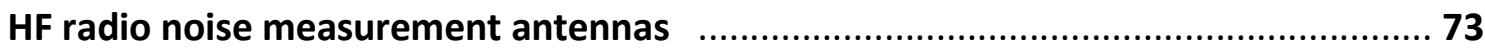

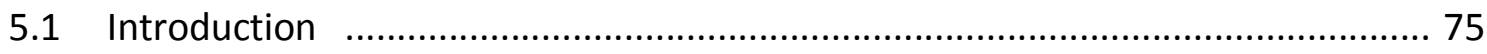

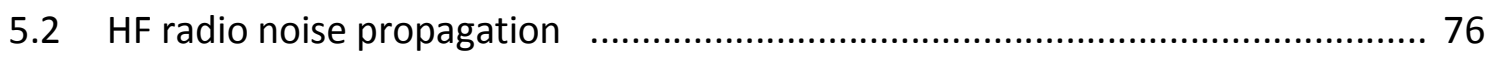

5.3 A novel method for the comparison of radio noise measurement antennas ..... 79

5.3.1 Short Lossless Vertical Monopole ...................................................... 79

5.3.2 Horizontal full-wave loop antenna ..................................................... 79

5.3.3 Inverted Vee half-wave dipole antenna ................................................ 82

5.4 Un-polarized omni-directional radio noise measurement antenna .................... 82

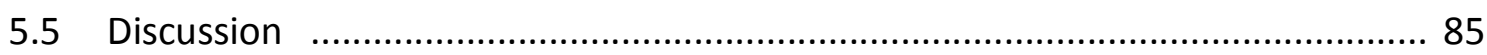

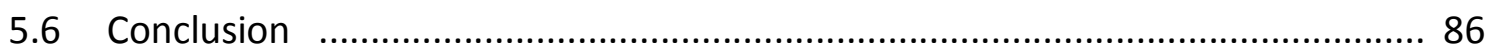

6 The importance of circular polarization for diversity reception and MIMO in

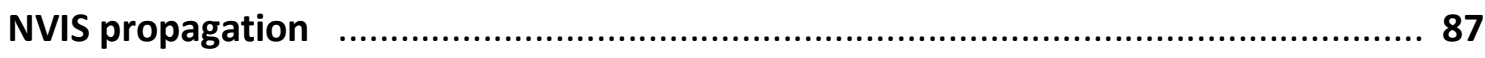

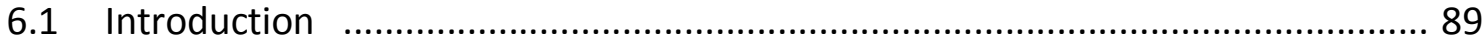

6.1.1 Circular polarization in NVIS propagation …..................................... 90

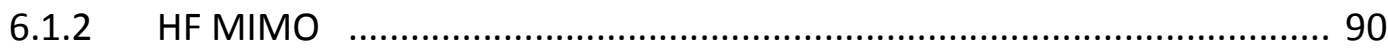

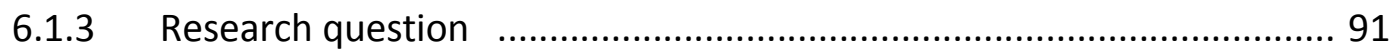

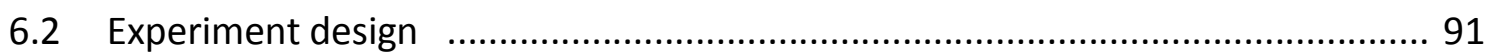

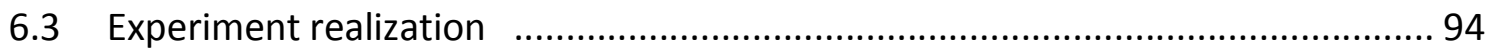

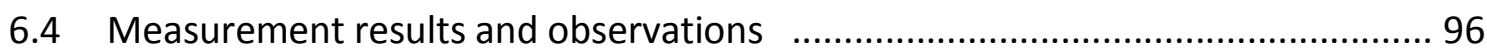

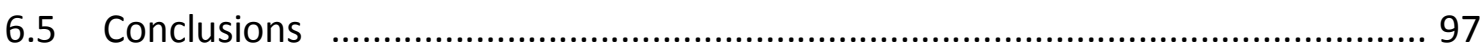

7 Measuring the Isolation of Circularly Polarized Characteristic Waves in

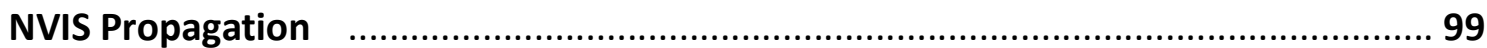

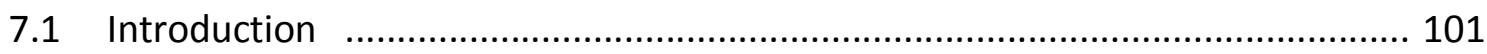

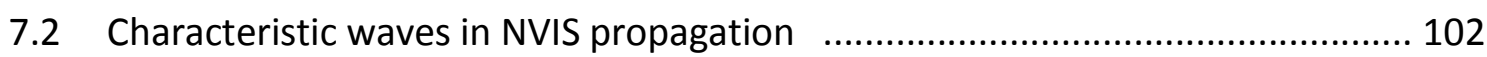

7.2.1 The 'Happy Hour' propagation interval ........................................... 103

7.3 Measuring NVIS characteristic waves isolation ........................................... 106

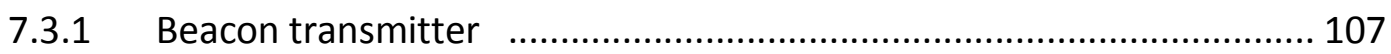

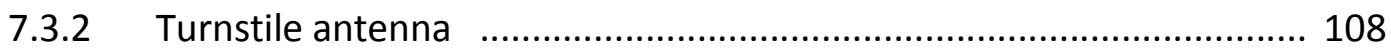

7.3.2.1 Balance transformers and feed lines ................................. 110

7.3.2.2 Phasing network ............................................................ 110

7.3.2.3 Antenna cross-polarization ................................................. 111

7.3.3 Measurement receiver ….............................................................. 112 


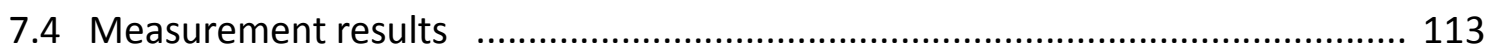

7.4.1 Signal identification and signal-to-noise ratio ....................................... 113

7.4.2 Dual circular polarization measurements ............................................ 114

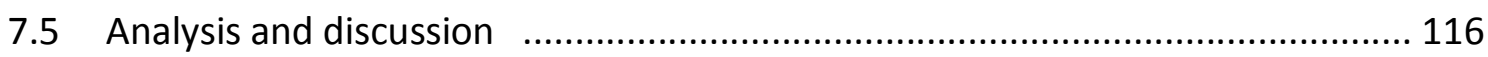

7.5.1 Morning Happy Hour observations ................................................. 116

7.5.2 Evening Happy Hour observations ….......................................... 118

7.5.3 Interpretation of measured characteristic wave isolation .................. 119

7.5.4 Nighttime propagation observations ............................................ 121

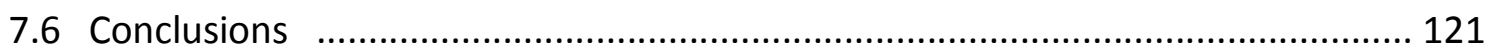

8 Characteristic Wave Diversity in Near Vertical Incidence Skywave propagation .... 123

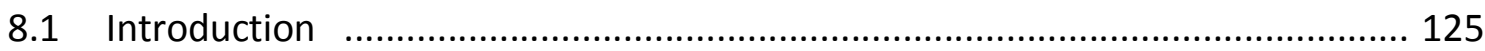

8.2 Circular polarized characteristic waves in Near Vertical Incidence Skywave .... 126

8.3 Characteristic wave diversity experiment ..................................................... 127

8.4 Reduction of the required fading margin ..................................................... 130

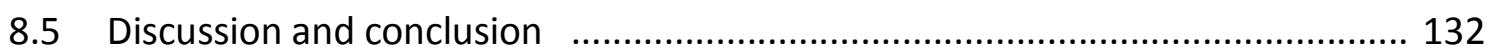

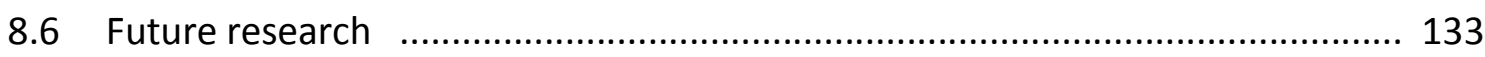

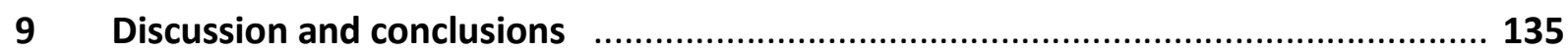

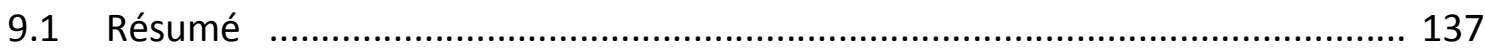

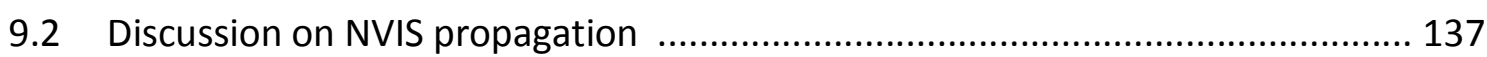

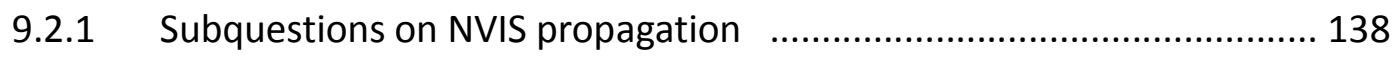

9.2.2 Research question on NVIS propagation ......................................... 140

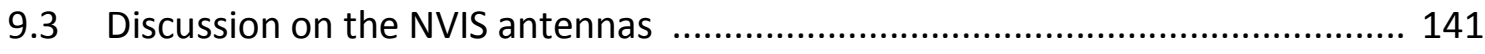

9.3.1 Subquestions on NVIS antennas f.................................................. 142

9.3.2 Research question on NVIS antennas .......................................... 143

9.4 Discussion on the interaction of antenna and propagation ............................. 143

9.4.1 Subquestions on the interaction of antenna and propagation ........... 144

9.4.2 Research question the interaction of antenna and propagation ........ 145

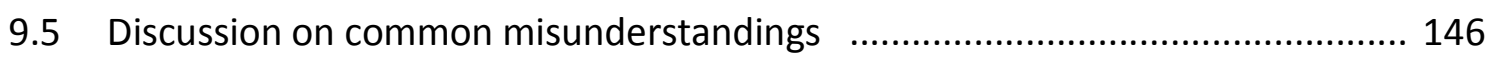

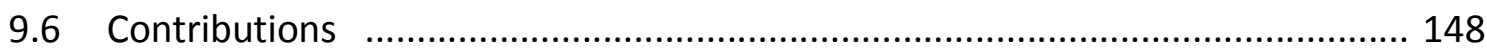

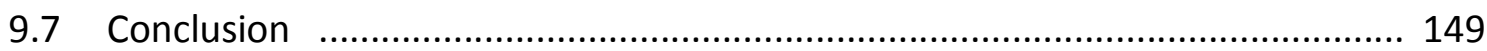

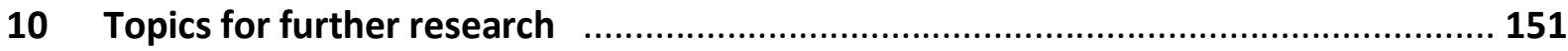

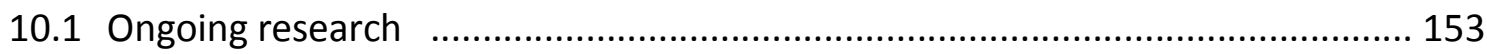

10.1.1 Path isolation experiments with dual circular polarization ............... 153

10.1.2 Nighttime above-the-MUF propagation measurements ................... 153

10.1.3 Improved circular polarization measurement equipment ................. 154

10.1.4 Planned NVIS experiments in the Netherlands and Spain ................. 155 


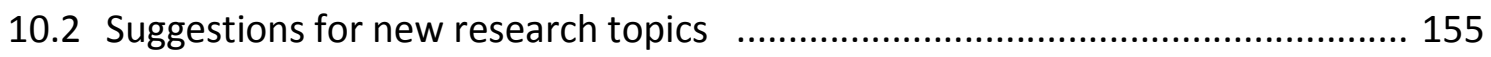

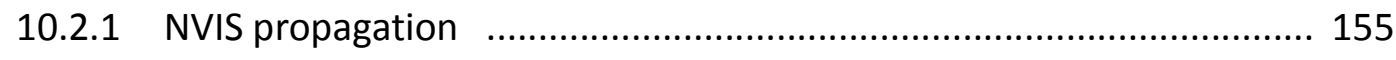

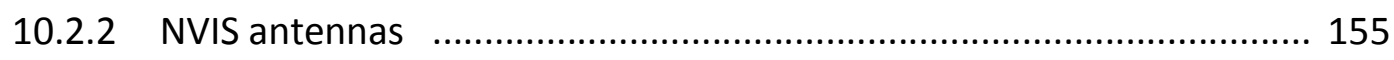

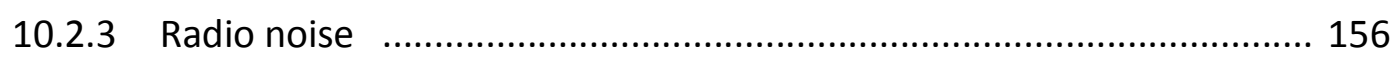

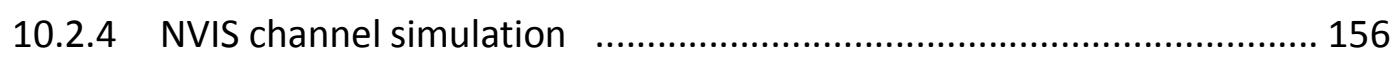

10.2.5 Modulation systems for NVIS channels ....................................... 156

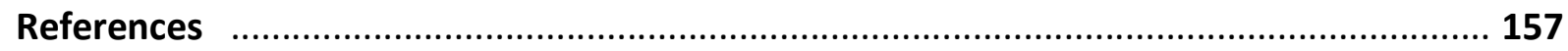

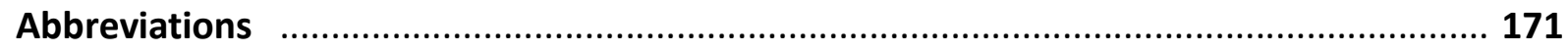

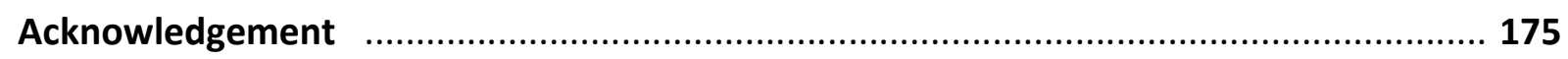

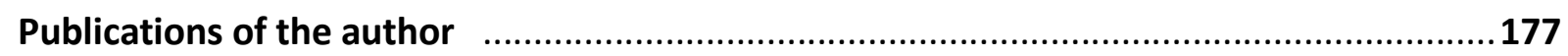

Author biography 
- xviii - 


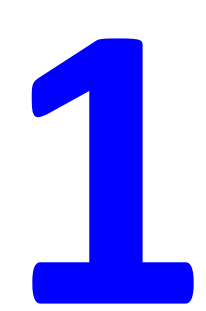

\section{Introduction}


Interaction of Antenna and Propagation Mechanism 


\subsection{A TELECOMMUNICATION DEPENDENT SOCIETY}

Our modern global society depends on telecommunication: communication beyond our physical horizon (the distance that can be reached with our own voice and gestures). Any process that needs up-to-date information from beyond that horizon, or depends on coordination with other processes occurring beyond that horizon, requires telecommunication. Near real-time telecommunication has become critical to most of our social, political and economic processes. Therefore we have created large, dense and technically advanced regional and national telecommunication networks, with long distance interconnections to virtually achieve global coverage.

\subsubsection{When the lines go down}

Generally we do not realize how fragile that connection to the society beyond our physical horizon is. When the telecommunication systems break down, such as is the case in large scale natural disasters, the impact on society is devastating [Comfort, 2006]. Without telecommunication, calls for medical help or practical assistance can no longer be conveyed, coherent status information from the stricken area cannot be gathered, and coordinated action in the disaster areas has become next to impossible [Miller, 2006, pp. 192-200]. The reports of a police officer in New Orleans after the flooding that followed hurricane Katrina, describing the disorientation and the total lack of coordination in the absence of telecommunication systems, provide a vivid impression of this phenomenon [Sims, 2007].

While the general image of our modern telecommunication systems is one of high reliability and redundancy, this is contradicted by the experience during large natural disasters. Reportedly, in such large scale natural disasters, the electrical power infrastructure and all telecommunication networks are disabled. At the same time roads are damaged and blocked by debris, making the installation of ad-hoc telecommunication systems difficult at best. [Kwasinski, 2006, 2009, 2011a, 2011b].

These disasters strike highly developed and undeveloped countries alike. We have seen a large number of examples in the last 10 years: the Indian Ocean tsunami in 2004, the flooding of New Orleans after hurricane Katrina in 2005 (see the cover of this thesis), the Haiti earthquake of 2010, the Japan Tohoku earthquake, tsunami and nuclear disaster in 2011 [Kobayashi, 2014] and the tropical cyclone Haiyan in the Philippines in 2013. For such large scale disasters, a telecommunication 'Plan B' should be prepared well ahead [Bodson, 1992], as will be discussed in Section 1.4. The use of HF (High Frequency: $3-30 \mathrm{MHz}$ ) ionospheric radio systems is one of the possible solutions [ITU Emergency Telecommunications Handbook, 2005, pp. 82-83], which has been reported to be effective in several occasions [Bodson, 1992; Tang, 2006; Lefeuvre, 2014]. Other alternatives often suggested are satellite telephone systems and (future) high altitude platforms. 


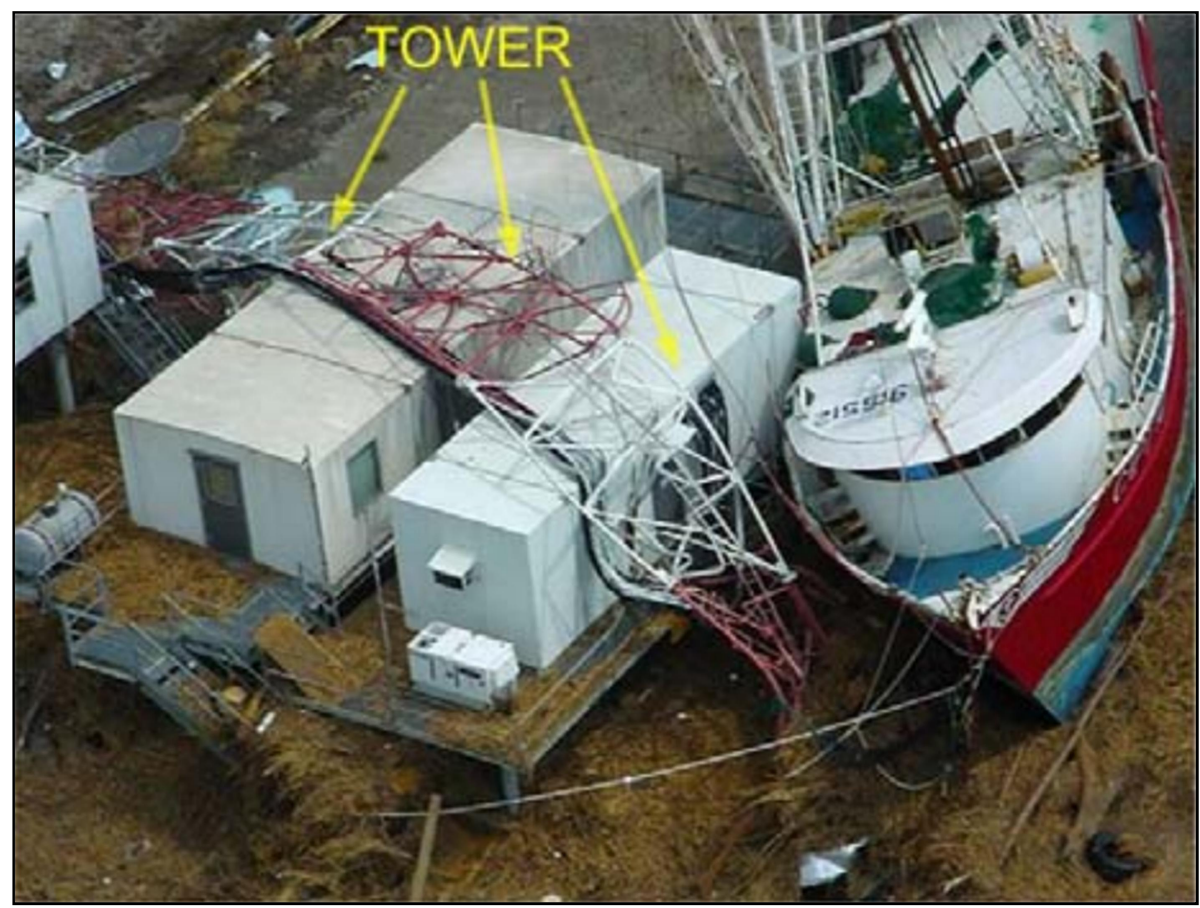

Figure 1.1 Aerial view of destroyed cellphone infrastructure in Venice, Louisiana, USA, after hurricane Katrina. Picture taken from [Kwasinski, 2006].

The narrowband HF radio communication systems cannot compete with the bandwidth offered by satellites. However, during the flooding of New Orleans in 2005, satellite telephones were unable to connect principal officials in the disaster area for a variety of reasons, ranging from expired subscriptions and unprogrammed equipment to antenna pointing problems and caller overload [Davis, 2006]. Also, when press coverage of the disaster started, so much satellite capacity was consumed by video news reports that the satellite telephones did not function anymore [McSwain, 2010]. Having stated this it must remain clear that - when equipment is available and the capacity distribution issue is solved satellite communications is of great value in disaster relief.

NVIS radio communication systems, on the other hand, can be deployed and used immediately, without the need of an intermediate operator. This is a critical success factor in the chaotic situation shortly after a large scale disaster strikes. Furthermore the associated antennas and radio equipment are comparably low-tech and are more easily built and maintained, even in countries with lower technological standard. This is also important in developing countries, in tele-medicine and tele-education as well as in in disaster relief efforts. Both NVIS and satellite communications should be seen as complementing each other, rather than competing, with a deployment preference depending on the local situation. 


\subsubsection{Areas without telecommunication infrastructure}

In the Western World we take our fast, reliable, comprehensive telecommunication infrastructure for granted. This makes us forget that in vast areas of the world no telecommunication infrastructure exists at all. And that there are other areas where some infrastructure is present, but its functioning is intermittent and unreliable.

In 1984, the UN Independent Commission for World-Wide Telecommunication Development [Maitland, 1984] signaled that large areas of the world were not connected to the Global Community at all, and that the lack of telecommunication infrastructure seriously hindered education and economic growth. Thirty years ago, in large parts of the African continent switched telephone networks were never established or, when established, very unreliable due to insufficient means to maintain them. Positive improvements were reported 20 years later [Milward-Oliver, 2005]. A rapid roll-out of modern wireless telecommunication networks is seen, integrating cellular phones, television and internet in daily life. However, this development is mainly in the bigger cities and the more prosperous regions of Africa. Observing the entire continent, only $0.1 \%$ of the people have cellular telephone coverage and the percentage of the total area covered is small [Buys, 2009], as depicted in Figure 1.2.

Also outside the African continent many regions remain where the population is too poor, the population density too low, or the terrain too forbidding for the installation of modern terrestrial telecommunication networks. In these areas stand-alone solutions for teleeducation and tele-medicine are sought that are very similar to the systems that can be deployed for first responders in disaster relief efforts [Martinez, 2004; Bandias, 2005]. Before the introduction of cellular telephone, private companies, banks, hospitals and governmental organizations in Africa depended completely on $\mathrm{HF}$ ionospheric radio for their telecommunication. In the Australian rural communities, HF radio has been used for the 'School in the Air', an educational system where the tutor visits the remote home by radio [Bandias, 2005].

\subsection{NEAR VERTICAL INCIDENCE SKYWAVE (NVIS)}

The coverage of a disaster area for first response relief efforts (Section 1.1.1) and the establishment of a simple but effective radio network for telecommunication in an area without infrastructure (Section 1.1.2) show great similarities. In both situations the network is to be as simple and as effective as possible. You want to establish contact immediately, without much alignment and testing, and especially without first having to establish cell towers or repeaters in the disaster area or in the remote terrain. The disaster area size and its topology dictate the choice of the communication system and associated propagation mechanism. When the area is limited in size and line-of-sight communication can be established from elevated structures or mountain slopes, VHF (Very High Frequency: 30-300 $\mathrm{MHz}$ ) or UHF (Ultra High Frequency: $300 \mathrm{MHz}-3 \mathrm{GHz}$ ) repeaters will work fine. 


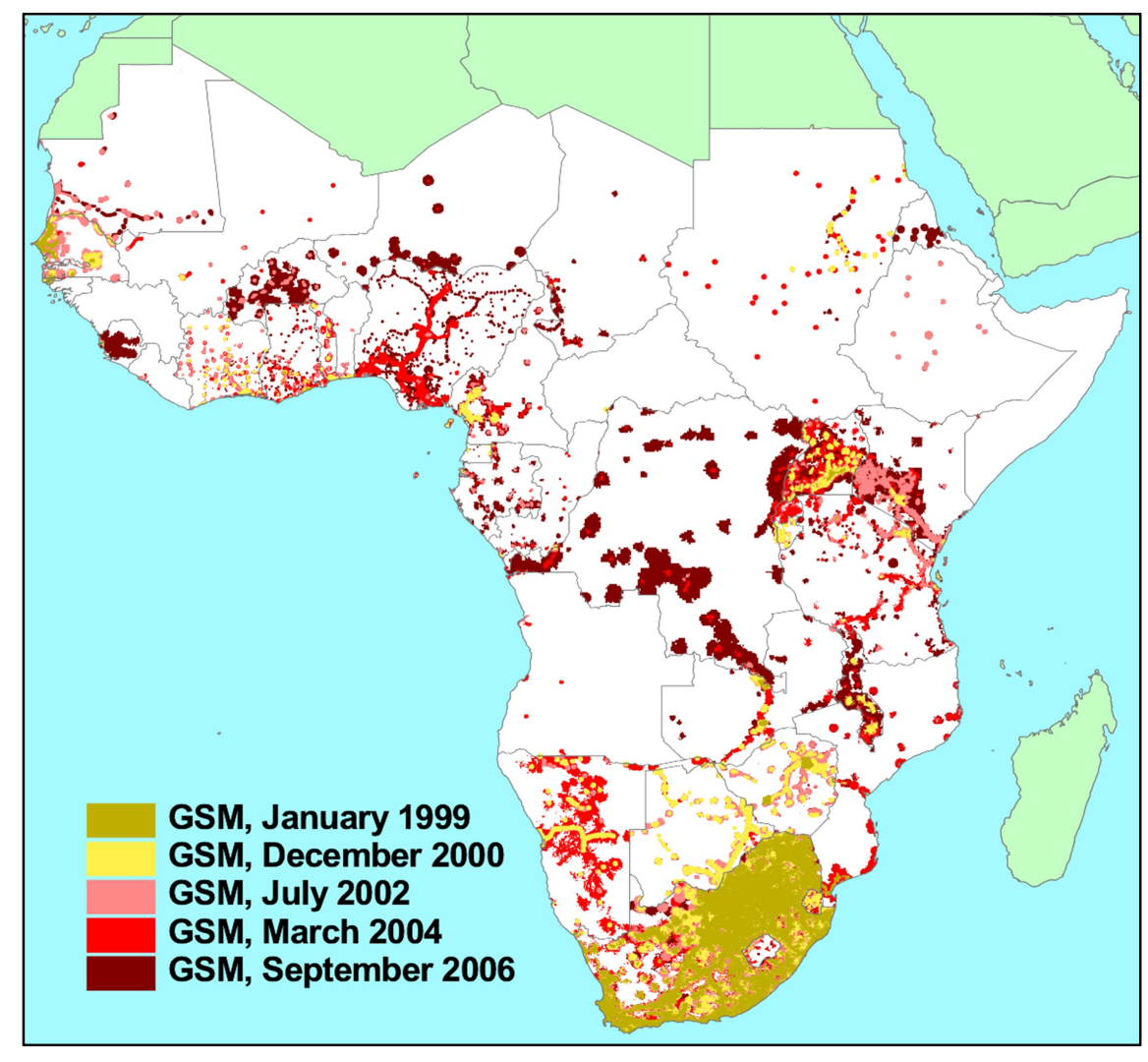

approx.

$7000 \mathrm{~km}$

approx. $7500 \mathrm{~km}$

Figure 1.2 Cellphone coverage in sub-Saharan Africa. Picture taken from [Buys, 2009]. Map dimensions added by the author to give an impression of the distances involved.

When the area is large, e.g. $400 \times 400 \mathrm{~km}$ as was the case with hurricane Katrina, line-of-sight communication systems will require placement of repeater installations within the disaster area itself, which may be impossible due to road and building damage [Kobayashi, 2014] and the lack of electrical power [Kwasinski, 2006]. If that is the case, ionospheric radio wave propagation offers an excellent alternative. When the frequency is properly chosen - typically between 3 and $10 \mathrm{MHz}$, as will be discussed in Chapter 4 - radio waves sent up towards the ionosphere at high angles are reflected at heights between 80 and $350 \mathrm{~km}$ to land in a large area around the transmitter. The antenna has to concentrate the transmit power upwards at high elevation angles, typically $70-90^{\circ}$, which explains the name of the propagation mechanism: Near Vertical Incidence Skywave (NVIS).

These steep angles bring another advantage in mountainous terrain or in between debris from collapsed buildings and structures: where line-of-sight systems suffer from shadowing effects from nearby mountain ridges or large objects, NVIS will provide coverage even in deep canyons [Johnson, 2007]. It will also work in the heavy rainfall typical after a tropical cyclone: rain attenuation at these frequencies is negligible. 
A single 100 Watt emergency communications base station with a simple dipole antenna will produce 35-55 dB signal-to-noise ratio (SNR), as will be shown in Chapter 9. This SNR does not gradually decay when the distance from the transmitter becomes greater, but is more or less constant over the entire $400 \times 400 \mathrm{~km}$ coverage area. Bitrates of up to $5.5 \mathrm{kbps}$ can be realized in a $2.4 \mathrm{kHz}$ bandwidth, provided the SNR is at least $17 \mathrm{~dB}$. This is sufficient for the transfer of text messages, weather charts, maps and medium-resolution photographs. Error resilient coding, handshaking and retransmission can provide an error-free data transfer and instantaneous confirmation of reception, properties that are important for emergency communications. Even a portable 1 Watt transmitter will produce sufficient SNR to maintain such a link. With proper frequency planning and used by skilled people, digital ionospheric radio can be as reliable as satellite communication [Goodman, 2005]. When used for teleeducation and tele-medicine in poor areas, the absence of the subscription fee and toll associated with satellite communication is important.

\subsection{OTHER APPLICATIONS OF NVIS}

NVIS communications also have a long history of military applications. Modern military telecommunications have moved towards high bandwidth solutions such as tropospheric scattering and satellite communications. Still NVIS has remained important as one of the communication alternatives, and its effectiveness has been improved by modulation systems optimized for the ionospheric channel, Automatic Link Establishment (ALE) and network protocols. HF radiocommunication - using both NVIS and long range ionospheric propagation - is still regarded as the communication backbone of Navy ships, and it is also used for telemetry on long distance commercial flights.

Medium wave and shortwave broadcasting in the tropical zone often exploits NVIS propagation. Also sea-going sailing yachts often employ digital modems and HF radio transceivers to send and receive radio mail and to obtain weather maps. When sailing closer to the shore $(<200 \mathrm{~km})$, the propagation mechanism involved is NVIS.

\subsection{THE IMPORTANCE OF NVIS RESEARCH}

NVIS propagation has been used for radio communication for a long time. Still the amount of scientific information on NVIS antennas and on the NVIS propagation mechanism is limited. Consequently, several opportunities to improve NVIS radio communication systems by scientific research remain, e.g. in the domain of antenna optimization, fading reduction and channel capacity enhancement. Also the empirical verification of theory is important; to find the blind spots in our present knowledge and to correct commonly accepted myths.

For emergency communications this research may be of life-saving importance. It is likely that in disaster relief the radio link will show asymmetry in sophistication. The field stations will probably be small, lightweight, with improvised antennas and operating on battery power. 
The radio stations that play a central role in the communication, e.g. the Emergency Communications Centers (ECC), will probably be located where electrical power is available, and will have more means to optimize their performance, which is necessary to compensate for suboptimal performance of the more improvised field stations. This optimization does not necessarily require large investments. More important is the knowledge of the propagation channel and the required adaptation of the equipment to it.

\subsection{MAIN RESEARCH GOAL}

Our main goal is therefore to improve NVIS telecommunication systems for disaster relief communication, tele-education and tele-medicine in terms of improved link reliability for the same amount of transmit power and for the same data transfer speed.

That goal is not pursued by designing an improved NVIS telecommunication system of specific characteristics, but rather by acquiring and transfering new knowledge on NVIS antennas, the NVIS propagation mechanism and the interaction between them both. Application of this knowledge is to improve NVIS telecommunication without high costs or great technological complexity, making application possible also in developing countries.

\subsection{FOCUS ON ANTENNAS AND PROPAGATION}

The focus of this research is on antennas and propagation, since most improvement to NVIS telecommunication systems can be achieved in this domain. As the ITU Handbook on Emergency Telecommunications [2005, p.99] states: "Time, effort and money invested in the antenna system generally will provide more improvement to communications than an equal investment to any other part of the station". To be precise: most improvement can be achieved by adapting the antenna properties to the propagation mechanism. In fact, the radio wave propagation in the ionosphere is altered by the antenna as well, in a way similar to the way that wave modes in a waveguide depend on the wave launcher. For best results, antenna and propagation have to be considered as a hybrid system. Research in this area will contribute most to improved NVIS performance, expressed in terms of achieved throughput versus required transmit power.

\subsection{STRUCTURE OF THIS THESIS}

The thesis is structured as a portfolio: the Chapters 4 to 8 contain the unaltered text of peerreviewed conference or journal publications. The overview article in Chapter 2 is in preparation for publication. The original summary and details of the publications are given on the title page of each chapter. Chapters 1, 3, 9 and 10 complement these publications with an introduction to the research, a description of the research question and a discussion of the research outcome. The outline is as follows: 
After the introduction in this chapter, Chapter 2 provides and overview of the most important aspects of an NVIS telecommunication system, identifies the subsystems that play a role in it, and discusses the interaction between these subsystems. The annotation in this chapter creates an extensive library of NVIS documents, details of which can be found at the end of the thesis. This library is recommended as a starting point for investigators starting research in the NVIS domain.

Chapter 3 identifies the research questions in the domain of antennas and propagation that will be addressed in this thesis.

In Chapter 4 the relationship between elevation angles and distance in NVIS propagation is derived from simulations and verified with measurements. Using these elevation angles, the optimum height-above-ground is sought for horizontal dipole antennas, which is then verified by measurement.

Chapter 5 describes a method to compare antennas used for the measurement of radio background noise, and their neutrality with respect to the spatial angle and wave polarization of the incoming noise.

Chapter 6 discusses the importance of characteristic waves for diversity and MIMO (Multiple Input Multiple Output) and shows by measurement that for mid-latitude NVIS these waves show left-hand and right-hand circular polarization. In Chapter 7 this experiment is repeated, but with significantly reduced measurement uncertainty and improved resolution.

Chapter 8 proposes a diversity reception system in which the polarization of the antennas is adapted to the polarization of the characteristic waves. The reduction of the multipath fading is examined using measurement data of the experiment described in Chapter 7.

In Chapter 9, the research results presented in previous chapters are used to answer the research questions that were formulated in Chapter 3, and topics for further research are suggested in Chapter 10. 
Interaction of Antenna and Propagation Mechanism 


\title{
2
}

\section{Radio communication via Near Vertical Incidence Skywave propagation: an overview}

\begin{abstract}
Near Vertical Incidence Skywave (NVIS) propagation can be used for radio communication in a large area $(400$ by $400 \mathrm{~km})$ without any man-made infrastructure. It is therefore especially suited for disaster relief communication, communication in developing regions and applications where independence of local infrastructure is desired, such as military applications. NVIS communication uses frequencies between approximately 3 and $10 \mathrm{MHz}$. In this chapter a comprehensive overview of NVIS research is given, covering propagation, antennas, diversity, modulation and coding. Both the bigger picture and the important details are given, as well as the relation between them.
\end{abstract}

Manuscript to be published: B. A. Witvliet, R. M Alsina-Pagès, M. J. Bentum, C. H. Slump , R. Schiphorst, "Radio Communication via Near Vertical Incidence Skywave Propagation - An Overview".

Sections 2.4 and 2.5 written by R. M. Alsina-Pagès, other sections written by B. A. Witvliet. 
Interaction of Antenna and Propagation Mechanism 


\subsection{INTRODUCTION}

Recently, interest in radio communication via Near Vertical Incidence Skywave (NVIS) propagation has revived, not in the least because of its role in emergency communications in large natural disasters that took place in the last decade [Straw, 2005; Lindquist, 2005; Ewald, 2006].

The NVIS propagation mechanism enables communication in a large area without the need of a network infrastructure, satellites or repeaters. This independence of local infrastructure is essential for disaster relief communications, when the infrastructure is destroyed by a large scale natural disaster, or in remote regions where this infrastructure is lacking. In military communications, where independence of local infrastructure is equally important, communications via NVIS propagation have always remained important next to troposcatter and satellite links.

For NVIS propagation, electromagnetic waves are sent nearly vertically towards the ionosphere, the ionized upper part of the Earth's atmosphere. With appropriate frequency selection, these waves are reflected back to Earth [Fiedler, 1996], as shown in Figure 2.1. The great reflection height of 80 to $350 \mathrm{~km}$ results in a large footprint, and due to the steep radiation angles large objects such as mountain slopes or high buildings cannot block the radio path [Austin, 1988]. Typical frequencies are between 3 and $10 \mathrm{MHz}$. NVIS propagation may be used to cover an area with a $200 \mathrm{~km}$ radius using low power and simple antennas [Fiedler, 1996]. The term 'Near Vertical Incidence Skywave' was first mentioned by Ruefenach and Austin in [Ruefenach, 1966], although others claim that Perlman [1970] named the propagation mechanism. The latter used the term 'Nearly Vertical Incidence Skywave'.

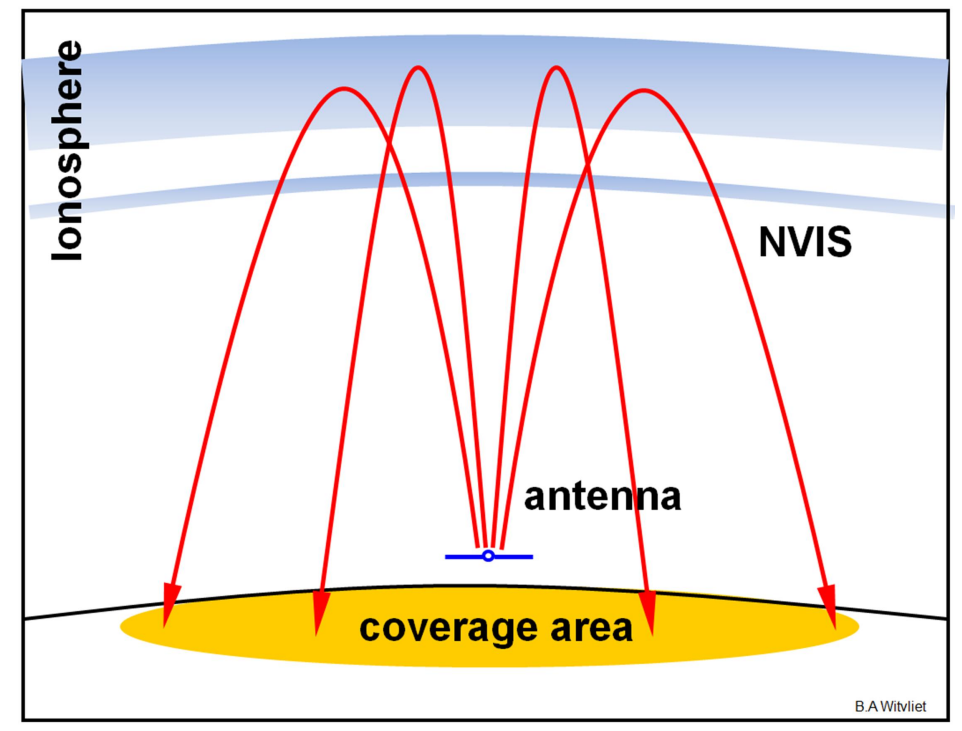

Figure 2.1 In Near Vertical Incidence Skywave (NVIS) communications, electromagnetic waves are sent nearly vertically towards the ionosphere. 


\subsubsection{Historical perspective}

The first documented scientific research into NVIS propagation was performed by Appleton and Bartlett [1924], to prove the existence of the 'Heavyside layer' by fringe measurements over $100 \mathrm{~km}$. In another experiment Appleton and Builder [1933] analyzes the difference in time delay between pulses transmitted over a distance of $5 \mathrm{~km}$, arriving both via groundwave and skywave. These experiments were performed to verify theories on radio wave propagation in the ionosphere. Vertical sounding has been used extensively for research into properties of the ionosphere since then, with increasing precision and sophistication.

Most ionospheric radio propagation research between 1930 and 1950 aimed at improving long distance telecommunications using 'short waves' (wavelength $<100 \mathrm{~m}$ ). Ionospheric radio communication proved very effective, and a world-wide public radiotelephony network was formed [Heising, 1940]. These HF radio links were gradually phased out when satellite transponders [Maunsell, 1964] and transatlantic cables with sufficient band width for telephony [Mervin, 1955] became available from 1956 onward.

NVIS (short distance) propagation was rediscovered in World War II as an essential means to establish communications in large war zones such as the D-Day invasion in Normandy [Fiedler, 1996, pp. 122-124; Austin, 2000], and a substantial volume of army sponsored research on NVIS field communications has been published since, especially between 1966 and 1973 [Ray, 1966; Hagn, 1973].

Modern radio and signal processing hardware enable new modulation and coding solutions, and automatic link establishment. The use of HF MIMO (Multiple Input Multiple Output) to increase channel capacity was first proposed by Strangeways [2006]. Research into the improvement of landmobile and airmobile NVIS antennas is also from the last two decades [Austin, 2002; Cummings, 2005].

\subsubsection{NVIS applications}

In the aftermath of a large scale natural disaster, often all telecommunication networks are disabled, the electrical supply is disrupted and the roads are blocked with debris or flooded [Kwasinski, 2006; Mikami,2012]. While our society is dependent on communication, total disruption of communication can have devastating results in the aftermath of a major disaster [Bodson, 1992; Sims, 2007]. NVIS communications have proven an excellent alternative for first responders in several recent large natural disasters, such as the Indian Ocean Tsunami of 2004 and the flooding of New Orleans after Hurricane Katrina in 2005 [Comfort, 2006a].

In humanitarian projects, NVIS propagation can provide low cost communications in poor and remote regions. A lot of progress has been made in connecting the developing world 
[Maitland, 1984; Ayeni, 2005], but areas remain where the telecommunication infrastructure is nonexistent, unreliable or inaccessible due to lack of financial means. In such regions voice and data traffic for tele-medicine and tele-education is often realized using NVIS propagation [Linden, 2004]. NVIS communications have always remained important as one of the alternatives in military communications. However, recent technological improvements have increased interest. Modulation systems are developed that are optimized for the ionospheric channel to increase data throughput, and modern Automatic Link Establishment (ALE) protocols [LeMasson, 2012] enable integration of NVIS links in heterogeneous communications networks.

While coverage in scientific media is scarce, medium wave and shortwave broadcasting in the tropical zone exploits NVIS propagation to cover the area around the transmitter in a power efficient way [Adorian, 1952]. Sea-going sailing yachts often also employ digital modems and $\mathrm{HF}$ radio transceivers to forward text messages and obtain weather maps.

\subsubsection{NVIS communication system optimization}

For disaster relief efforts, first responders in the field will have to work with battery power and improvised antennas. Similar power and antenna limitations may apply to humanitarian projects and military field operations. Optimizing the entire NVIS communication system may substantially (10-30 dB) reduce the required link budget. The block diagram of a MIMO NVIS communication system is given in Figure 2.2. It can be reduced to a diversity system by omitting the second modulator, transmitter and transmit antenna, or to a SISO (Single Input Single Output) system by omitting the entire second transmit-receive chain.

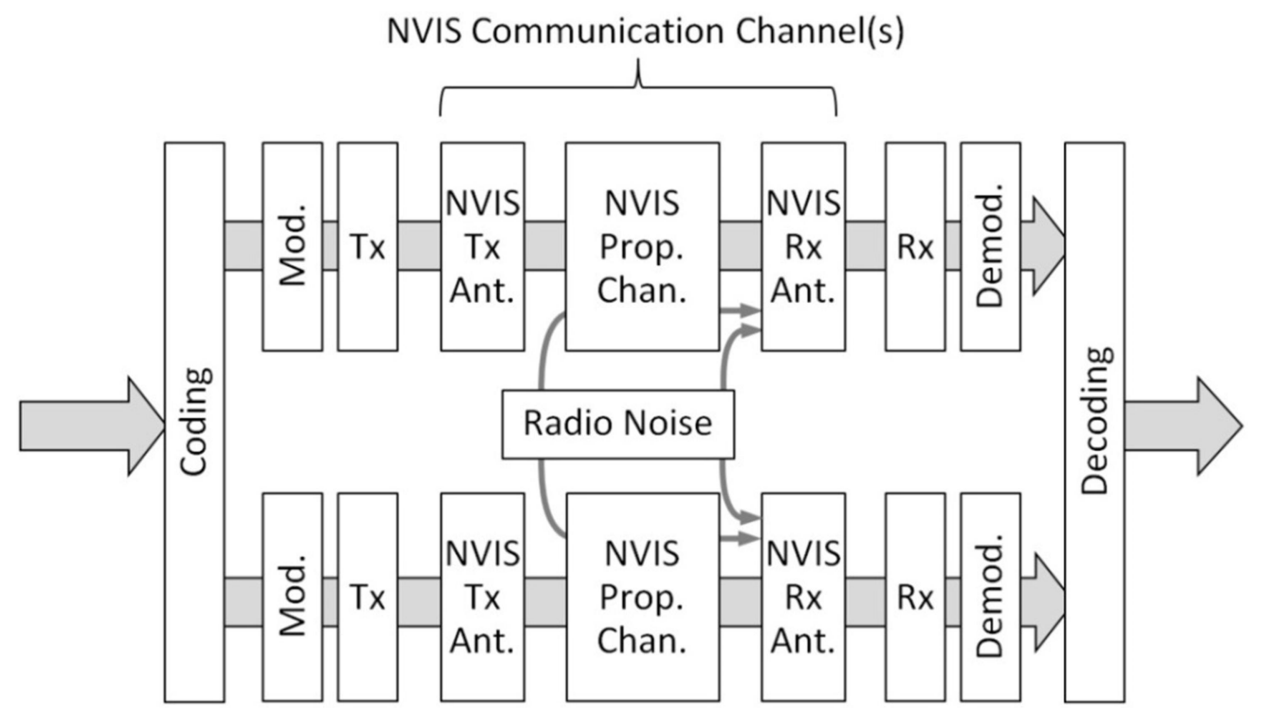

Figure 2.2 Block diagram of an NVIS communication system. Two propagation channels are shown, as in diversity and MIMO (Multiple Input, Multiple Output) systems. 'Mod.' and 'Demod.' stand for modulator and demodulator; 'Tx', 'Rx' and 'Ant.' stand for transmitter, receiver and antenna. 
System optimization requires research on antenna parameters, propagation mechanism, diversity, channel parameters, modulation techniques and coding. While specialization is needed for in-depth research in one of these fields, their interaction is substantial and needs to be considered when studying one single aspect. For example, the chosen antenna pattern and polarization influences channel fading and time dispersion, resulting in different coding and modulation optima. This chapter provides an overview of research relevant to NVIS communication systems, discussing the building blocks and the relations between. It also identifies niche subjects within the NVIS research field, where additional research will connect and augment other research and improve the overall knowledge of NVIS propagation and related systems.

This chapter is structured as follows: An overview of research on NVIS propagation and antennas in Sections 2.2 and 2.3. NVIS channel characterization and associated modulation and coding techniques are discussed in Sections 2.4 and 2.5. A discussion on subjects that merit more research and some concluding remarks can be found in Section 2.6.

\subsection{NEAR VERTICAL INCIDENCE SKYWAVE PROPAGATION}

\subsubsection{The ionosphere}

The radiation of the sun ionizes gasses in the upper part of the Earth' atmosphere: the ionosphere. Several ionospheric layers (regions) can be identified, each layer having its particular composition and being ionized by specific wavelengths in the solar radiation. In the F-layer atomic oxygen absorbs extreme UV radiation, with a peak at $175 \mathrm{~km}$ height. In the E-layer, between 90 and $150 \mathrm{~km}$ height, $\mathrm{O}_{2}$ absorbs soft $\mathrm{X}$-ray and UV radiation. The Dlayer, between approximately 60 and $90 \mathrm{~km}$ height, is caused by photo-ionization of NO molecules by Lyman-alpha radiation [Davies, 1990]. The D-layer, responsible for high attenuation at the lower HF frequencies, disappears almost completely at night. By daylight, the F-layer is split into a lower F1-layer and a higher F2-layer.

\subsubsection{NVIS propagation}

Electromagnetic waves entering the ionosphere may be refracted back to Earth, depending on the operating frequency. A wave traveling vertically will be reflected by one of the layers when its operating frequency is lower than the critical frequency of that layer. Radio waves with a frequency above the critical frequency will pass through the layer at vertical incidence, but will be reflected at lower elevation angles [Martyn, 1935], resulting in coverage starting at a certain distance of the transmitter, and a circular zone around the transmitter without coverage, as shown in Figure 2.3: the 'skip zone'. To realize a coverage area directly around the transmitter without such a skip zone, the operating frequency must remain below the critical frequency of the layer used. Both the E- and F2-layer can be used for NVIS links. 


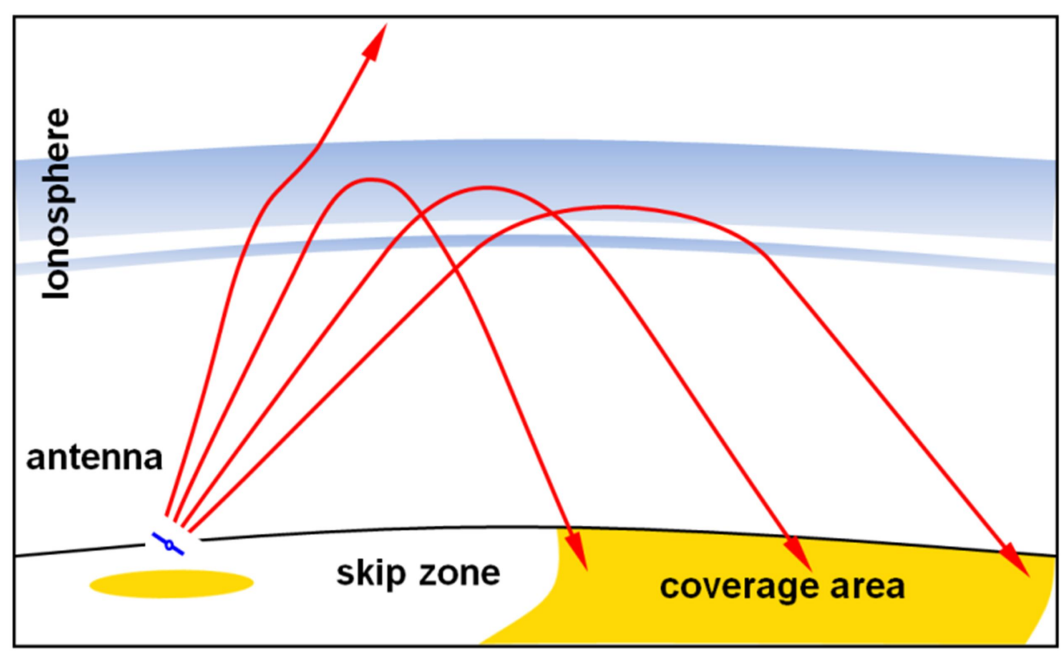

Figure 2.3 Transmission above the critical frequency of the ionosphere results in a 'skip zone'.

Absorption being lower at higher frequencies, F2-layer NVIS links will be more energy efficient [Davies, 1990]. Due to the large reflection height and relatively short distances covered, elevation angles are high.

\subsubsection{Characteristic wave propagation}

Experiments of Appleton [1933] show double reflections at the F1- and F2-layer, as we can see in Figure 2.4. Appleton mathematically proved that electromagnetic waves entering the ionosphere, under the influence of the Earth magnetic field, are split into two elliptically polarized characteristic waves with opposite rotation sense, the ordinary and extraordinary wave [Davies, p.82]. Appleton's magneto-ionic theory extended previous work of Maxwell and Thomson [Maxwell, 1873, pp. 404-408], who explained the polarization rotation found experimentally by Faraday in 1845 [Faraday, 1845; Knudsen, 1976]. This magneto-ionic propagation is treated in [Ratcliffe, 1962; Budden, 1985; Davies, 1990] and [Rawer, 1993]. The critical frequency of the ionospheric layer is different for each of these waves. Consequently, their path through the ionosphere is different, as can be shown with raytracing techniques [Reilly, 1991, 2000]. The waves suffer different attenuation and show different channel characteristics, such as delay and fading patterns. The polarization of the characteristic waves, as seen when entering or leaving the ionosphere, depends on the propagation of the waves with respect to the magnetic field. In the Northern hemisphere the polarization of the ordinary wave is right-hand circular (IEEE definition) on the upward and left-hand circular on the downward path. For mid-latitude (between $30^{\circ}$ and $60^{\circ}$ ) locations NVIS propagation at frequencies above $5 \mathrm{MHz}$, the polarization of the characteristic waves is nearly circular. Closer to the magnetic equator the polarization approaches linear (horizontal), at the magnetic poles it is circular [Davies, 1990, pp. 77-83]. 


\section{$\underbrace{G \quad F_{1}^{\prime} F_{1}^{\prime \prime} F_{2}^{\prime}} \quad G^{G} F_{1}^{\prime} F_{1}^{\prime \prime} F_{2}^{\prime} F_{2}^{\prime \prime}$

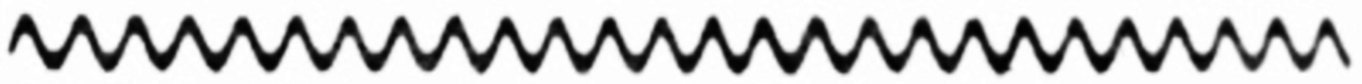

Figure 2.4 NVIS measurements by Appleton [1933]. Upper trace: pulses are received first via ground wave (G), then twice via the F1-layer (F1' and F1") and twice via the F2-layer (F2' and F2"). Lower trace: a 1115 Hz sine wave serving as time reference.

\subsubsection{Diurnal variation and solar cycle}

The solar energy absorbed in the ionosphere changes with the slant of the incoming sun rays, and the ionization of the ionosphere shows a diurnal variation [Chapman, 1939]. The critical frequency follows this pattern with a maximum near mid-day and a minimum early in the morning. Also, on mid-latitudes, the ionization changes with the seasons. On top of that, the radiation of the Sun varies over time following its sidereal rotation and its 11-year sunspot cycle [Davies, 1990, p. 130-136]. To maintain NVIS propagation, a frequency has to be selected that remains below the critical frequency of the F2 layer for a larger part of the day, to change to a lower frequency at night. Research on NVIS performance can be found in [Wagner, 1995] for high latitudes, and in [Farmer, 1996] for the specific case of low solar flux indices.

\subsubsection{Propagation prediction}

To gain insight in the actual NVIS propagation, measurements from the nearest ionosonde may be used. Observing the diurnal variation of the critical frequency of the F2-layer for the ordinary wave (foF2) and extraordinary wave ( $f x F 2$ ) over one or two sidereal days will give an indication of the propagation to be expected in the next few days. Johnson [2007] compares measured 24-hour NVIS link availability with VOACAP [Perkiomaki, 2003-2014] propagation predictions on several frequencies between 3 and $9 \mathrm{MHz}$ during a solar minimum. Walden [2009] reports that several propagation prediction models neglect the effect of the extraordinary wave. In [ITU-R Rec. P.434, 1995] basic recommendations and formula for ionospheric propagation prediction are provided, and in [Zolesi, 2014] a state-ofthe-art ionospheric prediction model is described. The combination of ionospheric parameters measured in real time and modeling ray-tracing software using the International Reference Ionosphere (IRI) model [Wilkinson, 2004; Bilitza, 2011] makes improved shortterm propagation predictions possible. 


\subsection{NVIS ANTENNAS}

As the ITU Handbook on Emergency Telecommunications [2005] states: "Time, effort and money invested in the antenna system will generally provide more improvement to communications than an equal investment to any other part of the station." Realizing this, several investigators published results on NVIS antenna optimization. Important parameters for NVIS antenna optimization are antenna radiation pattern, polarization and bandwidth. As only high elevation angles contribute to NVIS propagation [Black, 1995], optimizing the antenna radiation pattern for these elevation angles will increase the effectively transmitted power and improve the signal-to-interference ratio at reception. This section is organized in paragraphs on fixed, field expedient, mobile and receive antennas, each application imposing specific limitations to the antenna optimization. A paragraph on in-situ NVIS antenna measurement is added. The section concludes with the influence of the antenna characteristics on channel parameters.

\subsubsection{NVIS antennas for fixed installations}

With some variation between different sources [Fiedler, 1996; Hervas, 2013] practical NVIS operating frequencies range from approximately 3 to $10 \mathrm{MHz}$, corresponding with wavelengths of 30 to 100 meters. Therefore, if this entire frequency range is to be covered with high directivity and high efficiency, NVIS antennas will be large. In fixed installations this is acceptable: the effort in mechanical engineering is balanced by a large reduction in required transmitter power, as well as an improvement immunity to interference on reception. NVIS antennas suitable for fixed installations are the Delta antenna [Cones, 1950], the vertical Rhombic Antenna [Johnson, 1993, pp. 11.7-11.16], and the Log-Periodic Conical Spiral Antenna [Dietrich, 1969]. These antennas are often used in ionosonde installations because of their frequency independent behavior. The Conical Spiral Antenna provides circular polarization with high (30 dB) cross-polarization [Dietrich, 1969], its polarization sense being determined by the winding direction. In fixed military installations a vertically oriented Log-Periodic Dipole Antenna is also used [Fiedler, 1996, p. 48]. A derivative, the Log-Period Zig-Zag antenna, is described in [Witte, 2008]. Broadside arrays of multiple dipole antennas [Jones, 2014] may also be considered, but their frequency coverage is generally limited to one octave. The Conical Spiral antenna and the vertically Log-Periodic Dipole Antenna (LPDA) can be seen in Figure 2.5.

\subsubsection{Field expedient NVIS antennas}

For field expedient use, the antennas described above are not practical: their transport is cumbersome and installation time-consuming, in some terrain even impossible. However, simple and light wire antennas can provide good NVIS performance, alongside with the desired flexibility. 

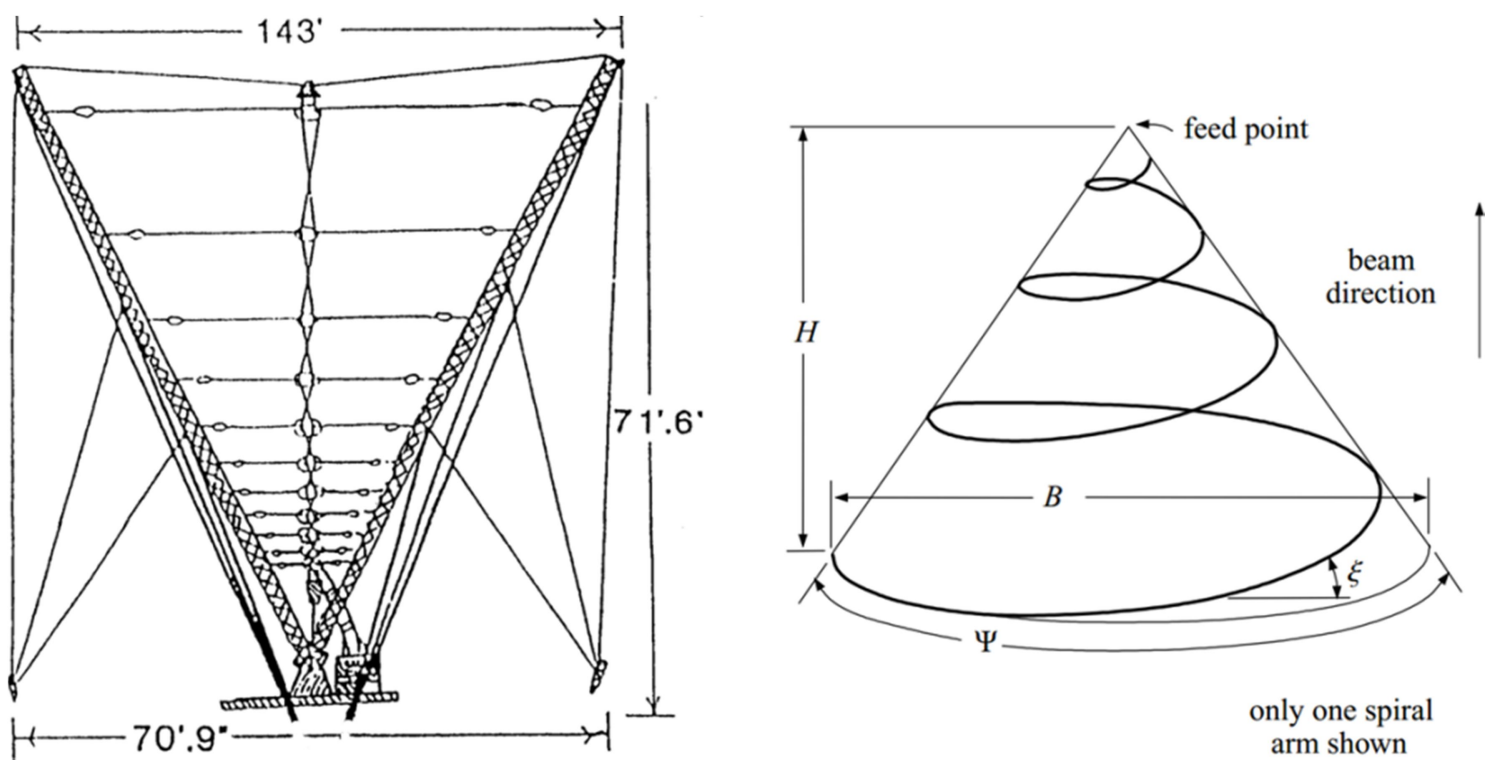

Figure 2.5 Two fixed NVIS antennas: (a) Vertical LPDA (b) Conical Spiral antenna. Figures taken from [Fiedler, 1996] and [Dietrich, 1969].

E.g. a simple wire dipole antenna may exhibit an antenna gain of approximately $6 \mathrm{dBi}$ at high angles, ground reflection included [Barker, 1971], and such an antenna may be suspended in between trees or from lightweight extendable fiberglass or aluminum masts. Extensive simulations and in-situ antenna pattern measurements on simple wire dipoles, Inverted L [Fiedler, 1996, p.50] and end-fed slanted wire antennas have been performed in [Barker, 1971]. This research included the influence of vegetation, antenna height and Earth magnetic field in California, USA and Thailand.

\subsubsection{NVIS antennas for mobile use}

Vertical whip antennas on vehicles perform badly in NVIS communications, as their radiation pattern shows a pronounced minimum at high elevation angles. Measured antenna gain at these angles range from $-35 \mathrm{dBi}$ at $4 \mathrm{MHz}$ to $-10 \mathrm{dBi}$ at $8 \mathrm{MHz}$ [Hagn, 1970]. Tilting the whip over the vehicle will only decrease the performance [Austin, 2002], and tilting the whip away from the vehicle is generally not possible when on the move. In [Austin, 1988] a carmounted vertical half loop antenna is described, using capacitance loading to achieve an NVIS antenna gain between $-12 \mathrm{dBi}$ and $-10 \mathrm{dBi}$ from $3 \mathrm{MHz}$ to $8 \mathrm{MHz}$. This antenna is depicted in Figure 2.6. Similar NVIS loop antennas have been designed for transport aircraft [Cummings, 2005], helicopter [Richie, 2003] or ships. Due to its increased size, the shipboard loop in [Vlasic, 2008] achieves an NVIS antenna gain between $-1 \mathrm{dBi}$ and $+4 \mathrm{dBi}$ between 2 $\mathrm{MHz}$ and $7 \mathrm{MHz}$. In helicopters, the antenna design has to consider modulation of the signal by the rotating rotors [Polycarpou, 2000; Richie, 2003]. 

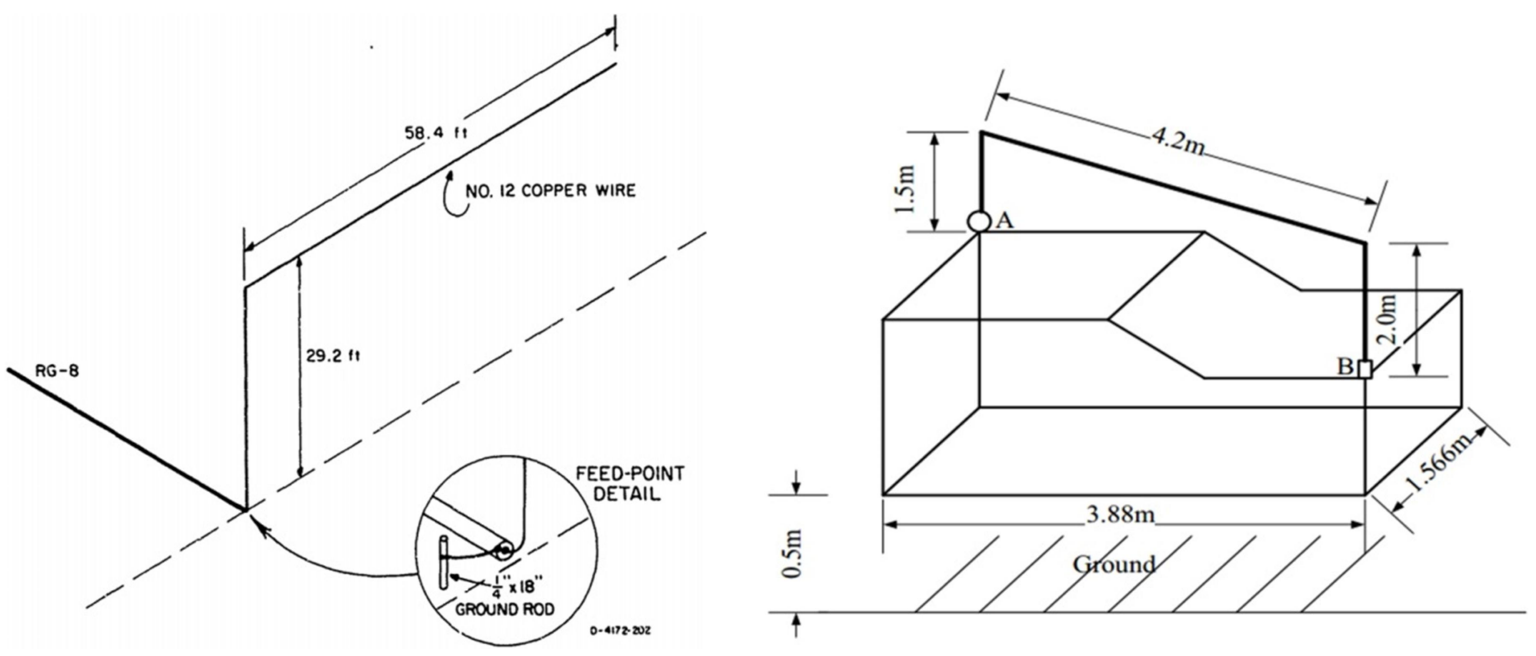

Figure 2.6 A field-expedient Inverted L antenna, and a mobile car loop NVIS antenna. Figures taken from [Barker, 1971] and [Austin, 2002].

\subsubsection{Antennas for NVIS reception}

Most of the publications presume that the same antenna is used for both transmission and reception. This is not necessarily the best solution. For reception the average directivity over the NVIS elevation angles is more important than the antenna gain [Kraus, 1988, pp. 766767], as the reception is limited by the ambient electromagnetic noise [ITU-R Rec. P.372, 2013] unless receiver sensitivity is very poor. Discrimination of the receive antenna radiation pattern between the NVIS elevation angles and angles at which most interference and ambient noise arrives [Coleman, 2002] may significantly improve reception. While unsuitable for transmission, very compact active antennas may provide maximum - ambient noise limited - sensitivity [Ellingson, 2005]. Directive arrays may be composed of such active antenna elements and angular filtering can be used to improve the signal-to-noise ratio on reception [Warrington, 2000].

\subsubsection{In-situ NVIS antenna measurement}

Due to their size, NVIS antennas cannot be measured in anechoic chambers. This is also true for the smaller mobile antennas, as their supporting platform is an integral part of the radiating structure. Also the influence of the ground underneath the antenna installation is not negligible and has to be included in the measurement. However, in-situ measurement of antenna pattern and relative gain for antenna evaluation can be achieved using a small transmitter transported by helicopter [Breakall, 1994], airplane [Jenkins, 1995], tethered balloon [Austin, 1988] or remote controlled octocopter drone [Krause, 2013]. Comparison of antenna performance using real-live NVIS transmitters is impossible due to fast and deep fading imposed by ionospheric propagation, unless specialized techniques are used. In [Hagn, 1973] an ionosonde is used to compare the antenna gain of two antennas at zenith angle using pulsed measurements. 


\subsection{NVIS CHANNEL CHARACTERISTICS}

In this section NVIS channel sounding and modelling, is described, including their variation with latitude. Several tests have been conducted in the United Kingdom by Burgess [1999] and Tooby [1999, 2000] to characterize the NVIS channel. Their results can be used to simulate the influence of the channel, enabling performance testing of modulation and coding systems without on-air measurements [ITU-R Rec. F.520, 1992]. Channel simulation also enables comparison of modulation and coding systems in identical circumstances, which is not possible with real propagation.

\subsubsection{NVIS channel sounding}

In order to design the modulation and coding system that minimizes BER with maximum throughput for any ionospheric link, a sounding system covering the entire HF band should be designed, as was done in [Villella, 2008] and improved in [Ads, 2012], in this case for a long distance link. Real data from Lowell ionosondes [Lowell, 2014] has been used by Hervás [2013] to characterize the NVIS channel. This data can be used to trade-off BER and bit rate when designing modulation and coding techniques for an HF NVIS channel. Other research uses Digital Radio Mondiale (DRM) broadcast signals [Digital Radio Mondiale, 2009] and the background radio noise measurements to calculate the parameters with which the best channel availability is obtained [Losada, 2009; Gil, 2012]. Previous research of [Tooby, 1999] used a chirp sounder to measure the signal-to-noise ratio (SNR) and the multipath characteristics between 1998 and 1999.

\subsubsection{Fading}

lonospheric propagation is prone to signal fading. Figure 2.7 shows the slowly changing ionospheric reflectivity and absorption on a timescale of tens of minutes, with superimposed fast and deep multipath fading, caused by interference between signals arriving via ionospheric paths of different length [ITU-R Rep. 266, 1990]. Multipath fading causes fast and deep (down to $-30 \mathrm{~dB}$ ) fades on a timescale of seconds. For the design of communication systems, knowing the mean value of the received signal is insufficient; the fading has a pronounced effect on the data reception. An extra signal margin has to be reserved for the fading minima, the 'fading margin', and the coding has to be adapted to allow for temporary symbol loss. McNicol [1949] discusses fading on vertical incidence signals between 2 and $6 \mathrm{MHz}$.

Fading time series are usually studied and modeled using stochastic processes [Brennan, 1961; Essex, 1968]. It is assumed that the fading generated by the composition of multiple ionospheric echoes can be represented as the sum of the specularly reflected signals plus a random component; this is modelled by the Nakagami-Rice distribution [Nakagami, 1960]. 


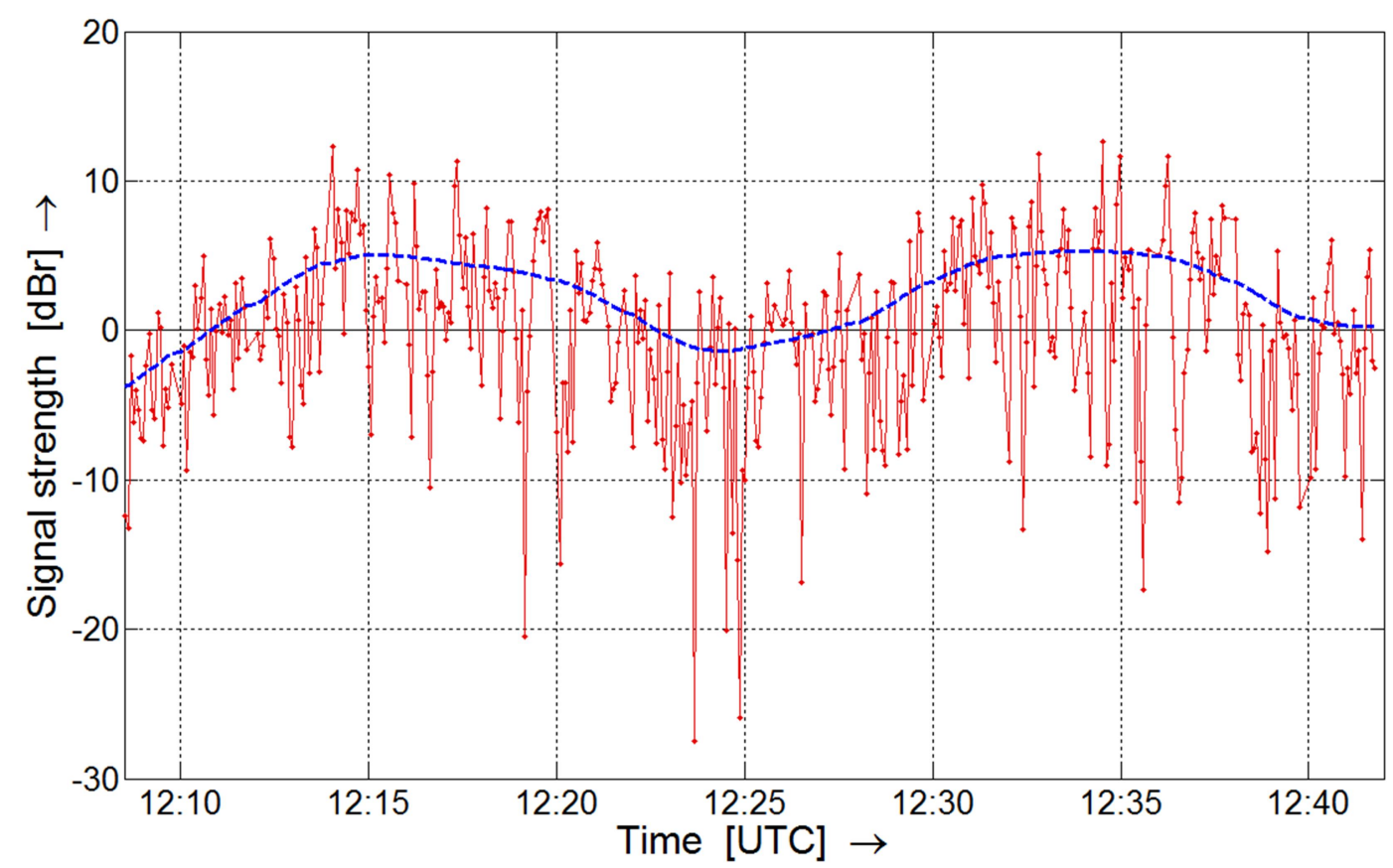

Figure 2.7 NVIS measurement, showing fast deep multipath fading (in red) superimposed on slower flat fading (blue line).

If the random component dominates, this probability function approaches a Rayleigh distribution [Papoulis, 2002]. According to [Davies, 1990, p. 237], short observations of ionospheric fading will reveal Rayleigh fading, while longer observations will show a lognormal signal distribution.

\subsubsection{Doppler}

Not only the depth of the fading is important, but also its period of appearance. Fading rates are often expressed in terms of the autocorrelation of the times series. This autocorrelation measures the speed with which a point in the time series is decoupled from its neighbors. This time difference measured by autocorrelation is usually called 'coherence time' [Proakis, 1995]. The coherence time has a direct relationship with the Doppler spread of the channel [Proakis, 1995]. Channels with large Doppler spread have signal components that change in phase over time, and since the fading depends on whether the addition of components is constructive or destructive; these channels have short coherence time. The coherence time has to be taken into account when choosing the symbol time of the modulation, to assure that the channel has an approximately constant response during the symbol.

\subsubsection{Delay spread}

The delay spread is a measure of the multipath of a communications channel [Proakis, 1995]. In general, it is interpreted as the time difference between the arrival of the earliest multipath component and the arrival of the latest multipath component. It is usually obtained by measuring the power delay profile of the channel [ITU-R Rec. P.1407, 2013]. 


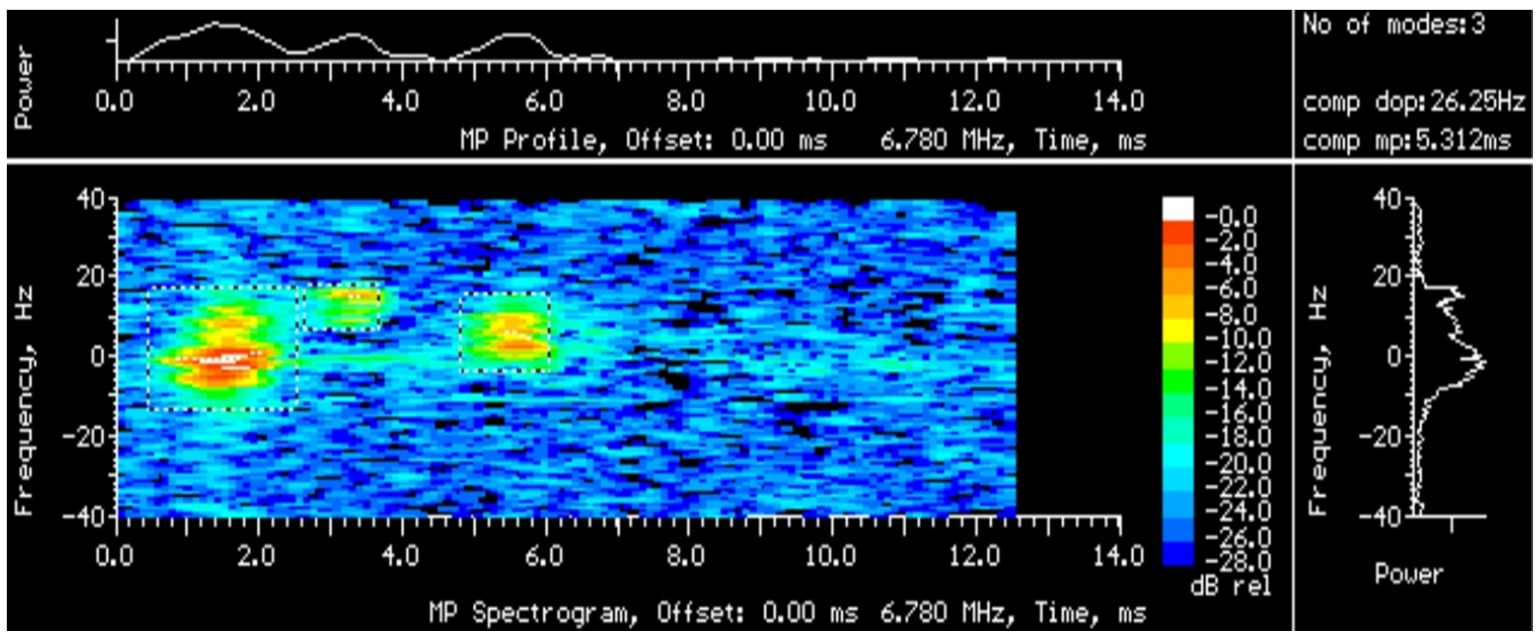

Figure 2.8 Doppler and multipath on a high latitude path in Scandinavia, measured with DAMSON (Doppler And Multipath SOunding Network). Figure adapted from [Cannon, 2005].

Figure 2.8 shows both Doppler and Delay Spread of a high latitude path in Scandinavia [Cannon, 2005]. The delay spread may cause Inter Symbol Interference (ISI). When designing a modulation system, the symbol duration must be long enough - usually 10 times the delay spread - so that an ISI-free channel is obtained. Equalization may be used to reduce the delay spread and multicarrier modulation may be used to increase the per carrier symbol duration. Delay spread in the time domain is linked to 'coherence bandwidth' in the frequency domain, which is a statistical measure of the range of frequencies over which all spectral components are transferred with approximately equal gain and linear phase [Sklar, 1997].

\subsubsection{NVIS channel modelling}

For many years, the standard ionospheric channel model for performance evaluation of $\mathrm{HF}$ communication systems has been the model of Watterson [1970], which is also described in ITU-R Rec. F.1487 [2000]. While this model properly simulates the signal perturbations such as Doppler and delay spread occurring in an interval of a few seconds, it does not represent the variations of the channel quality, especially the SNR, occurring in longer intervals in the range of a few seconds to 10 minutes. In [Furman, 2009], both long-haul and NVIS measurements are performed to model these SNR variations to improve the Watterson model. Some work can be found in the study and modelling of the channel characteristics using polarization diversity techniques to improve the system performance [Hervas, 2013]. Finally, measurements on broadcast signals in the medium wave (MW) band are used to improve the prediction accuracy for network planning in that band [Gil, 2012, 2013], providing the first channel model for NVIS propagation in the medium wave band [Guerra, 2013]; a tapped delay line (TDL) model based on field measurements. 


\subsubsection{NVIS channel characteristics as function of latitude}

Several propagation reference books [Budden, 1985; Davies, 1990; Hunsucker, 2002] describe the physical phenomena in the ionosphere. In terms of HF communications, ionosphere studies can be divided into three zones: i) polar zone (high-latitude), ii) midlatitudes and iii) equatorial zone (low latitude) [Magdaleno, 2011], each of them with its own particularities.

Wagner [1995] is one of the first to characterize the high-latitude ionospheric NVIS channel. Warrington [2006] and Jodalen [2000], amongst others, also provide a thorough channel analysis for high latitudes NVIS paths. Jodalen [2000] presents the results from a propagation experiment over two NVIS paths in northern Scandinavia using DAMSON (Doppler and Multipath Sounding Network), with the aim of correlating the results for both paths. Warrington [2006] presents direction finding measurements in Norway and Sweden, to better understand the directional characteristics of HF signals reflected in the high-latitude ionosphere. Lossman [2011], who performed tests in the Baltic region, concluded that transmission frequencies greater than $5 \mathrm{MHz}$ show higher effective data speeds due to the fact that man-made noise is mostly concentrated between 2 and $5 \mathrm{MHz}$. In [Tooby, 1999], the quality of mid-latitude NVIS channels is measured, evaluating SNR and multipath data. Hervás [2013] performs mid-latitude NVIS channel soundings using a Lowell Digisonde [Lowell, 2014], obtaining information about Doppler and multipath delay spread, and the best possible transmission frequency as function of the hour of the day. And finally, in [Cannon, 2000] several channel evaluation tests were conducted to compare data from the equatorial region of the ionosphere with the auroral propagation using DAMSON, reaching the conclusion that wideband HF broadcast can achieve high availability when operating on fixed frequencies.

\subsection{MODULATION AND CODING FOR THE NVIS CHANNEL}

\subsubsection{Data transmission protocols for NVIS communications}

Several ITU recommendations contain information on fading and its impact on modulation systems [ITU-R Rep. 266, 1990], on delay spread evaluation [ITU-R Rec .P.1407, 2013] and on modem testing [ITU-R Rec. F.1487, 2000]. These documents are informative only, leaving the implementation to the designer.

Hoult [2000] presents a study to achieve $16 \mathrm{kbps}$ data transmission speed in a standard 3 $\mathrm{kHz}$ wide HF channel, a significant improvement compared with the 2.4 or $4.8 \mathrm{kbps}$ that were available at the time. It uses an NVIS channel instead of a VHF link, as the latter could not be used due to the accidented terrain. Several other studies were done to improve the effective bitrate of modems used in one-hop ionospheric propagation and NVIS propagation 
[Jodalen, 2001]. Johnson [2006] details modem characteristics for a wireless mesh network for NVIS propagation, long-haul skywave and surface wave signals.

Some of the HF Data Transmission Protocols, e.g. STANAG 4415 [NATO, 1999], MIL-STD 188110C [US Department of Defense, 2011], and DRM [Digital Radio Mondial, 2009], are designed to work for both NVIS and oblique incidence propagation, and are designed for faster data transfer in the presence of fading, multipath and noise. In [Jodalen, 2001], the link availability of two modems - one compatible with STANAG 4415 and 4285 [NATO, 1989], the second with STANAG 4285 only - has been compared with Morse code and voice. [Davies, 2003] contains measurements of throughput in relation to SNR for STANAG 4538 [NATO, 2009] over several distances, to test the effects of low SNR and variable channel conditions, taking into account advanced waveforms, coding and protocols.

In [Johnson, 2006] two alternative channel access technologies are tested and compared in wireless mesh networks: Automatic Link Establishment (ALE) and fixed-frequency MAC protocols. In [Furman, 2012] we find measurements on a MIL-STD-188-110C link over distances of up to $160 \mathrm{~km}$, providing the users with bit rates up to $9.6 \mathrm{kbps}$ in 6 to $9 \mathrm{kHz} \mathrm{RF}$ bandwidth. The standard gives the option of selecting bandwidth and modulation, including wideband modulation with up to $24 \mathrm{kHz}$ bandwidth with elaborate coding and interleaving. It must be noted, however, that standard $\mathrm{HF}$ channels are only $3 \mathrm{kHz}$ wide.

Commercial HF radio modem protocols such as PacTor-IV claim to achieve $5.5 \mathrm{kbps}$ in 2.4 $\mathrm{kHz}$ RF bandwidth at $17 \mathrm{~dB}$ SNR and to maintain link down to $-20 \mathrm{~dB}$ SNR [SCS, 2011]. PacTor has proven successful in disaster relief communication and is used for radio mail and weather map transfer for sea sailing yachts, but scientific comparison with other protocols is not available.

DRM, an international radio broadcasting standard launched in 2003, solves fading and phase distortion problems associated with shortwave AM broadcasting and offers both increased audio quality and bandwidth [Losada, 2009]. Spreading the information in frequency and time over multiple carriers in OFDM modulation makes the system less prone to selective fading. Coding and interleaving are implemented and their parameters can be selected to match the expected propagation channel. An option for NVIS is included. In the thesis of Losada [2009] a study of the application of DRM is detailed, evaluating the statistical performance of the modem in different environments (e.g. Doppler, delay, interference).

\subsubsection{Experimental modulation and coding}

The following research was done outside the previously cited standards. Hoult [2000] describes the design of a $16 \mathrm{kpbs}$ modem in $3 \mathrm{kHz}$ wide NVIS channel and shows results in both laboratory and field tests. In [Antoniou, 2006] there is a study of several modulation techniques tested over a distance of $160 \mathrm{~km}$ on frequencies between $2.8 \mathrm{MHz}$ and $9.4 \mathrm{MHz}$, 
presenting measured SER (Symbol Error Rate) values for data rates from 4.3 to 20.6 kbps. More details of this work can be found in [Christofi, 2001]. Finally, [Lamy-Bergot, 2013] presents over the air transmissions using an HF modem operating over multiple noncontiguous $3 \mathrm{kHz}$ channels spread over a $200 \mathrm{kHz}$ wide subband.

\subsubsection{Diversity and MIMO}

Reducing fading and increasing throughput on an HF channel can also be realized using diversity techniques. Frequency diversity, time diversity, antenna (spatial and polarization) diversity and adaptive beamforming to suppress unwanted multipath were invented in the early 1930's, and implemented in intercontinental HF radio communication systems applied [Beverage, 1931; Friis, 1937].

At the technological level of that era this required great ingenuity and considerable expenses. Today's RF and signal processing hardware allows compact and cheap realization of elaborate diversity systems, promising substantial reductions in required link budget. Diversity system may be integrated in the coding software on both ends of the link (MIMO), in selection software after the demodulator (selection diversity), or in the baseband of the receiver (e.g. optimum ratio combining).

\subsection{SUBJECTS THAT MERIT MORE RESEARCH}

A large amount of research has already been done on individual components of the NVIS radio communication system, but several subjects remain that merit investigation, to augment or interconnect the existing research. The following niche subjects were identified that merit further investigation:

\subsubsection{Antennas and Propagation}

Empirical research into the nature of polarization fading, together with measures to reduce this fading. Documented observations of the presence of multipath fading in a single hop single layer NVIS propagation path - with polarization fading excluded - would trigger new research on the nature of fading mechanisms within a single layer.

For the optimization of NVIS antennas, generally only the antenna gain at zenith angles is considered. A new approach is needed for such optimizations. Depending on the antenna type, the desired coverage area size and the ionospheric reflection height, the radiation in a range of spatial angles must be considered. For that purpose, graphs relating elevation angle to great circle distance provided McNamara [1991] and Davies [1996, Fig. 12.2] could be improved and extended to show the dependency on the operating frequency and the sunspot number. Using this new antenna optimization approach, comparison of a large number of NVIS antenna types, preferably for several coverage area sizes, and accompanied 
by their optimum dimensions and optimum installation heights would be valuable as reference material for NVIS antenna selection.

Also the difference between antenna performance on transmission and reception is not covered yet and merits a closer look, as HF reception is limited by ambient electromagnetic noise. Verification of theoretical and simulated antenna optimizations under live NVIS propagation conditions are desirable to validate these optimizations. Due to the signal fading that is typical for ionospheric propagation, and as the fading patterns may differ for each antenna, antenna comparison requires a new approach. Also experiments with antennas with polarization matched to the characteristic waves in the ionosphere could bring new insights and applications. Compact antennas to produce adapted or adaptive polarization would be an asset for such experiments.

\subsubsection{Channel, modulation and coding}

Channel simulators for F2-layer and E-layer NVIS links for several bandwidths are helpful to compare and improve modulation and coding protocols. These models should incorporate selectable and combined ordinary and extraordinary wave channels for the design of diversity and MIMO systems. Moreover, if the real NVIS channel response could be recorded for several minutes and reproduced, this would provide an invaluable tool for the comparison of protocols in realistic yet repeatable circumstances.

Comparison of a large number of standardized HF communication protocols - including PacTor - over identical NVIS channels would allow comparison and analysis of the effectiveness of different solutions in modulation, coding, and adaptive behavior.

Open (non-proprietary) modulation, coding and data transfer protocols are needed, to encourage further development by third parties. Protocols designed to achieve consistent high data rates in the complex NVIS channels are much sought after, but high data rates are not always priority. Research into low speed protocols achieving highly reliable data transfer with acknowledgment at very low SNR are invaluable to transfer lifesaving information when circumstances (antenna situation, battery power, propagation) are unfavorable. Diversity and MIMO systems may increase data throughput at low SNR values.

\subsubsection{Interaction of building blocks}

Research into the interaction of building blocks such as antenna, propagation, modulation system and coding, may prevent that isolated optimization of single blocks leads to a suboptimal overall design. 


\subsection{CONCLUSION}

An overview is given of the main building blocks of an NVIS radio communication system. The text provides references to a large cross section of the existing literature on NVIS radio communication systems, to provide new researchers in this field with an initial NVIS library. However, it does not pretend to be exhaustive. Investigators are encouraged to contribute to NVIS research, especially in the areas indicated in the previous Section, to contribute to increased reliability of NVIS emergency communication systems. 
Interaction of Antenna and Propagation Mechanism 
Research questions and research outline 
Interaction of Antenna and Propagation Mechanism 


\subsection{RESEARCH QUESTIONS}

As was explained in Section 1.5 and 1.6, our main goal is to improve NVIS telecommunication systems by acquiring and transferring new knowledge on NVIS antennas, the NVIS propagation mechanism and the interaction between them both. In Chapter 2 we identified several NVIS research subjects that were not covered sufficiently yet, of which several in the domain of antennas and propagation, our research focus. The following research questions were formulated:

\section{Question 1:}

How does the NVIS propagation mechanism function, and what parameters of this mechanism are important for NVIS telecommunication system optimization?

Motivation:

Understanding the mechanism that underlies NVIS propagation is crucial for any attempt to improve the interface between the antenna and the propagation mechanism. Empirical data is very important in propagation research, and this is especially the case in propagation in complex media such as the ionosphere, producing better insight in optimization goals and possible solutions.

\section{Question 2:}

How can we optimize the NVIS antenna to (a) produce the strongest signal across the coverage area, and (b) to realize the greatest signal-to-noise ratio on reception of signals from that coverage area?

\section{Motivation:}

The answers of research question 1 may be used as input to optimize NVIS transmit and receive antennas. While NVIS antenna optimizations have been studied before, the differentiation between transmit and receive properties has not received sufficient attention, and other aspects of matching the antenna to the propagation mechanism may have been missed.

\section{Question 3:}

How important is the interaction between NVIS antenna and NVIS propagation mechanism?

\section{Motivation:}

If interaction between the antenna and the propagation mechanism is negligible, the problem formulated in research question 2 simplifies to a simple antenna optimization. If this interaction is not negligible, a more holistic approach is needed, and the antenna and propagation mechanism must be considered to be a hybrid system. 


\subsection{RESEARCH SUBQUESTIONS}

For each of the research questions, several subquestions can be formulated, which will help to direct the research.

\section{Subquestions concerning NVIS propagation are:}

Subquestion 1-1: Is it possible to realize a continuous coverage area of $400 \times 400 \mathrm{~km}$ using a single 100 Watt HF base station?

Subquestion 1-2: What frequencies should be used and what propagation mechanism is involved?

Subquestion 1-3: What is the role of ground wave propagation?

Subquestion 1-4: How much power is needed to realize coverage in the entire coverage area for at least $16 \mathrm{kbps}$ throughput in a $3 \mathrm{kHz}$ bandwidth?

Subquestion 1-5: What is the influence of multipath fading on the required link budget?

Subquestion 1-6: What is the relationship between the elevation angle of the transmitted waves and the distance covered? What other parameters are involved in this relationship?

Subquestion 1-7: What role does antenna polarization play in NVIS propagation?

\section{Subquestions concerning NVIS antenna systems are:}

Subquestion 2-1: Which antenna parameters must be considered for optimization?

Subquestion 2-2: Is there a difference between transmit and receive antenna optimization?

Subquestion 2-3: What role does electromagnetic background noise (radio noise) play in reception?

Subquestion 2-4: How does the angular distribution of radio noise influence antenna optimization?

\section{Subquestions concerning the interaction of antenna and propagation mechanism are:}

Subquestion 3-1: In what ways do the transmit antenna properties change the propagation of the radio waves in the ionosphere?

Subquestion 3-2: In what ways must the receive antenna be adapted to the ionospheric radio wave propagation?

Subquestion 3-3: Must we consider the combination of antenna and propagation mechanism as a hybrid system with a mutual dependency?

Subquestion 3-4: How can knowledge of the NVIS antenna-propagation interaction be used to reduce the necessary transmit power or to increase the data throughput? 


\subsection{COMMON MISUNDERSTANDINGS}

When discussing NVIS antennas and propagation with professional NVIS users, knowledge about the propagation mechanism and the necessary antenna systems is often intermixed with commonly accepted misunderstandings (myths) that undermine optimal application of what is known. Therefore, in addition to these research questions, the credibility of the following assumptions are examined:

Assumption 1: The NVIS antenna must be suspended at low heights above ground to give best radiation towards the zenith and hence best NVIS performance.

Assumption 2: The signal-to-noise ratio (SNR) of an NVIS signal received on a dipole antenna suspended close to the ground is much better than the SNR on a dipole that is suspended higher above ground.

Assumption 3: Vertical whips on cars may be poor NVIS performers, but if you tilt the whip by 45 degrees, performance is restored.

Assumption 4: Short HF radio links - up to and maybe even beyond $100 \mathrm{~km}$ distance depend on ground wave propagation and require a vertically polarized antenna.

Assumption 5: At night, on frequencies where the ionosphere does no longer support NVIS, signals arrive via ground wave.

Assumption 6: Waves propagating through the ionosphere have linear polarization when launched from linearly polarized antennas.

\subsection{OUTLINE OF THE RESEARCH}

The research consists of three major parts, described below, each looking into a specific aspect or a few related aspects of NVIS antennas and propagation. Each part contains at least one set of field measurements, in which data is gathered using real ionospheric propagation for comparison with theory and simulations. The empirical content is considered important in this research, and much attention is given to measurement accuracy and experiment precision.

\subsubsection{Elevation angle versus distance}

In the first part of the thesis, described in Sections 4.1 to 4.3 , the path of the radio wave through the ionosphere is simulated using ray-tracing software to obtain the relationship between the elevation angle and the distance covered. To test these simulations against reality, the elevation angle of the signals of 85 transmitters arriving via NVIS propagation is measured on two frequencies, 3.5 MHz and $7 \mathrm{MHz}$, using an HF Radio Direction Finder. These measurements are used to obtain an empirical relationship between elevation angle and distance. From the measurement data, the distance at which NVIS propagation becomes dominant over ground wave can be derived. 


\subsubsection{Antenna performance versus height above ground}

The second part investigates the optimization of the antenna radiation pattern using the elevation angles found in Chapter 4. As most NVIS antennas are used at heights below one wavelength above the ground, the influence of ground absorption and reflection is included. A distinction is made between transmit antennas, where the antenna gain is of importance, and receive antennas, where the achieved SNR matters.

To enable optimization for the entire coverage area, 'NVIS Antenna Gain' and 'NVIS Directivity' are defined. The optimum height above ground for horizontal half-wave dipoles used as transmit and receive antenna are found for several soil types by simulation.

For verification, the performance of 5 antennas suspended at different heights is measured, using the signal of a beacon transmitter arriving via NVIS propagation. Both relative antenna gain and relative SNR are compared with theory. This research is described in Sections 4.4 to 4.6.

The apparent non-uniform distribution of ambient electromagnetic noise or 'radio noise' that was noted during this research triggered an investigation into the sensitivity of radio noise measurement antennas with respect to angle of arrival and polarization. This research is described in Chapter 5.

\subsubsection{The role of radio wave polarization}

The third part of the research investigates the role of radio wave polarization, and the influence of antenna polarization on reception. It is found that only left-hand and right-hand circularly polarized waves are observed in NVIS propagation at mid-latitudes.

In two experiments the isolation (absence of crosstalk) between the characteristic waves is measured. The experiments exploit the two special propagation intervals that occur daily, one in the morning just after the NVIS propagation has begun, and one in the evening just before the NVIS propagation ends. As theory and ionospheric ray-tracing simulations show, only one of the characteristic waves is received during this interval. This was predicted by Davies [1990], but not observed yet.

The first experiment - described in Chapter 6 - is performed to prove the general concept. The second experiment - described in Chapter 7 - uses an improved measurement system with better measurement accuracy and more polarization discrimination. These measurements are also used to evaluate the advantage of using antennas with polarizations matched to the characteristic waves in polarization diversity systems. This is described in Chapter 8.

Using these investigations, the research questions that were formulated in Chapter 3 are discussed in Chapter 9. New research questions triggered by these results are collected in Chapter 10. 


\title{
Near Vertical Incidence Skywave propagation: elevation angles and optimum antenna height for horizontal dipole antennas
}

\begin{abstract}
Near Vertical Incidence Skywave (NVIS) communication uses the ionosphere as a reflector to cover a continuous area with a radius of at least $150 \mathrm{~km}$ around the transmitter, on frequencies typically between 3 and $10 \mathrm{MHz}$. In developing countries - in areas lacking any other telecommunication infrastructure - it is used on a daily basis for voice and data communication. It may also be used in ad-hoc emergency (disaster) communication in other regions. This chapter proposes optimum heights above ground for horizontal dipole antennas for NVIS, based on simulations and empirical data. Firstly, the relationship between elevation angle and skip distance is obtained using ionospheric ray tracing. The high elevation angles found by simulation are confirmed by elevation angle measurements using a professional Radio Direction Finder. The measurements also show the dominance of NVIS over ground wave propagation starting at a short distance. For these elevation angles, the optimum receive and transmit antenna heights above ground are derived using antenna simulations. A distinction is made between optimum transmit signal strength and optimum received signal-tonoise ratio. These optima are verified experimentally, demonstrating a novel evaluation method that can be used in the presence of the fading typical for ionospheric propagation. For farmland soil ( $\sigma \approx 20 \mathrm{mS} / \mathrm{m}, \varepsilon_{\mathrm{r}} \approx 17$ ) the optimum height above ground for the transmit antenna is 0.18-0.22 $\lambda$. If the antenna is lowered to $0.02 \lambda$ a transmit signal loss of $12 \mathrm{~dB}$ occurs. This corresponds with the theory. The receive antenna height, however, while appearing uncritical in the simulations, showed a clear optimum at 0.16 $\lambda$ and a 2-7 dB SNR deterioration when lowered to $0.02 \lambda$.
\end{abstract}

Published as: B. A. Witvliet, E. van Maanen, G. J. Petersen, A. J. Westenberg, M. J. Bentum, C. H. Slump, R. Schiphorst, " Near Vertical Incidence Skywave Propagation: Elevation Angles and Optimum Antenna Height for Horizontal Dipole Antennas," in IEEE Antennas and Propagation Magazine, Vol. 57, No. 1, Feb. 2015. 
Interaction of Antenna and Propagation Mechanism 


\subsection{INTRODUCTION}

Near Vertical Incident Skywave (NVIS) radio wave propagation uses the ionosphere as a reflector, on frequencies ranging from approximately 3 to $10 \mathrm{MHz}$. While HF (High Frequency) radio communication has ceded its role in daily European and North American life to satellite communication and cellular networks, it still thrives in more challenging adhoc situations, such as disaster relief and military operations [Wallace, 1992]. NVIS propagation is also used on a daily basis in developing countries - in areas where telecommunication networks are unreliable or non-existent - providing essential telecommunication, such as voice and data communication between small business offices, healthcare facilities and even banking facilities. It may also be used on an ad-hoc basis in disaster relief communications, providing a quickly deployable alternative to destroyed telecommunication infrastructure in natural catastrophes such as the 1953 'Big Flood' in The Netherlands [Rollema, 2004; Gerritsen, 2005], the 2005 flooding of New Orleans and the 2011 tsunami in Japan. To cover an area around and directly adjacent to the transmitter, electromagnetic waves must be launched at steep angles, entering the ionosphere nearly perpendicularly, hence the prefix "Near Vertical Incidence". In the ionosphere, the electromagnetic waves are reflected back to earth after which they land in an umbrella-like fashion in the area around the transmitter, as is illustrated in Figure 4.1.

\subsubsection{The NVIS antenna}

The NVIS antenna - probably the most important element in the radio link - may consist of a simple wire structure and can be cheap and efficient, provided sufficient knowledge is available to engineer and install these antennas optimally. Optimizing the antenna radiation pattern for NVIS elevation angles promises significant improvement of the radio communication link.

The traditional vertical whip antennas on cars do not perform very well when using NVIS propagation due to the null in their antenna diagram at high elevation angles [Austin, 2002]. [Hagn, 1970] discuss measurements on whip antennas on military vehicles. For best NVIS performance they propose tilting the whips in a horizontal or slanted position when stationary. They arrive at effective antenna gain values between -17 and $-35 \mathrm{dBi}$ on frequencies from 4 to $8 \mathrm{MHz}$, which is still quite poor. For mobile NVIS applications loop antennas are better adapted, although, due to their small size, their instantaneous bandwidth and efficiency are limited at low frequencies. The optimization of the vertical radiation diagram of such antennas remains a challenge, due to the radiation of currents induced in the vehicle body [Austin, 1994]. On large helicopters, these currents may cause unwanted rotor modulation on specific frequencies [Richie, 2003]. On the other hand, these current can be used effectively by creating an NVIS slot antenna in the body of an airplane [Saakian, 2012]. A large shipboard loop is described in [Vlasic, 2008]. 


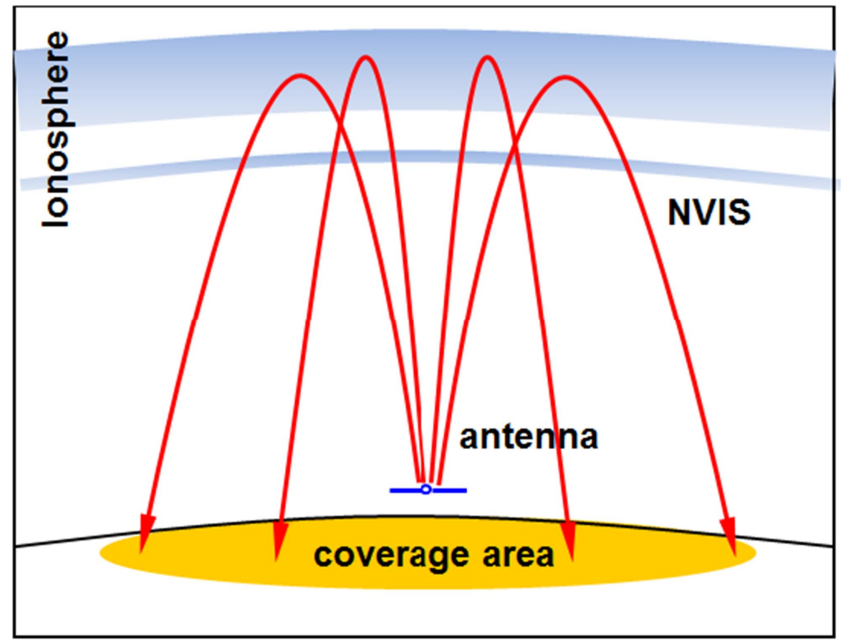

Figure 4.1 Near Vertical Incidence Skywave (NVIS): electromagnetic waves launched nearly vertically are reflected back to earth after which they land in an umbrella-like fashion in the area around the transmitter.

When more installation time is available, wire antennas such as dipoles may be used to provide better performance. Research into NVIS field antenna performance has been performed in the 60's and 70's by [Barker, 1971] in the USA and in the tropical rainforest of Thailand. A RF source towed by an airplane was used to compare the shape of the radiation pattern with simulations [Barker, 1973] and the relative antenna gain at the zenith was compared using an ionospheric sounder [Hagn, 1973]. [Austin, 1994] used a helium filled balloon ('blimp') for NVIS antenna measurements.

\subsubsection{NVIS reception}

NVIS receive antennas must be optimized for best signal-to-noise ratio (SNR) rather than for best antenna gain. Normally, at HF, external noise dominates over receiver noise, defining the reception threshold. Predicted levels for atmospheric, galactic and man-made noise can be found in ITU-R Rec. P.372-11 [2013]. As the levels of man-made noise are highly dependent on electric and electronic equipment quality, equipment density and geographical distribution, and while the latter have changed over time, new HF noise measurements campaigns using modern means [Van Maanen, 2006] are desirable. Interesting studies show the non-uniform azimuthal distribution of noise [Posa, 1972; Coleman, 2002], which is important for the understanding of HF receive antenna signal-tonoise performance.

The central topic of this chapter is the optimization of transmit and receive antennas for fixed or temporary base stations that use NVIS radio wave propagation. The research concentrates on horizontal half-wave dipole antennas for NVIS coverage of an area with 150 $\mathrm{km}$ radius, the area of a mid-sized European country or US state. 
The following contributions are made:

- The relationship between NVIS elevation angle and coverage distance is investigated using ionospheric ray tracing software.

- Elevation angle measurements are performed, involving 85 NVIS stations, proving the dominance of NVIS over ground wave starting at short distances and confirming the high elevation angles involved in NVIS propagation (70-90 $)$.

- NVIS Antenna Gain and NVIS Directivity are defined, to facilitate NVIS antenna comparison. Optimum antenna heights are proposed for different soil types, based on antenna simulations.

- A novel empirical evaluation method for NVIS antenna performance in the presence of HF fading is introduced and demonstrated. These measurements confirm the optimum transmit antenna heights found by simulation.

- However, the optimum height for the receive antenna (highest signal-to-noise ratio) does not conform to the simulated values.

This chapter is structured as follows: Section 4.2 provides a brief summary on ionospheric radio wave propagation. In Section 4.3, the relationship between NVIS elevation angle and skip distance is investigated using simulations, which is then verified by experiment.

Section 4.4 discusses the adaptation of NVIS antennas to the properties of NVIS propagation. The research then limits itself to horizontal half-wave dipole antennas above real (lossy) earth, and the influence of the antenna suspension height. A differentiation is made between transmit and receive performance.

Section 4.5 introduces a novel empirical method that allows evaluation of NVIS antenna performance in situ and using NVIS propagation. Practical implementation of this method is discussed, identifying possible pitfalls and describing practical enhancements that improve accuracy. This method is used to verify the optima that were found by simulation and the measurement results are discussed in paragraph 4.5 .3 (for the transmit case) and paragraph 4.5.4 (for reception).

Section 4.6 compares the optima found with other research and discusses the applicability of the results to other frequencies, other coverage area size and other sunspot numbers. The chapter concludes with a summary of the results and subjects that were identified for further research.

\subsection{IONOSPHERIC RADIO WAVE PROPAGATION}

To optimize the NVIS antenna we have to look into ionospheric radio wave propagation first. An extensive overview on the formation of the ionosphere and radio wave propagation through it can be found in [Budden, 1985; Davies, 1990; Solar Terrestrial Dispatch, 2001]. 
We will limit ourselves here to a brief summary of the subject, with a focus on the key parameters that are linked to NVIS antenna optimization. The ionosphere extends from an altitude of approximately $50 \mathrm{~km}$ upwards to several Earth radii [Davies, 1990]. The ionization is caused by UV, X-ray and Ly- $\alpha$ radiation from the sun, balanced by ion depletion due to recombination and diffusion. The resulting vertical ion density profile was first described by [Chapman, 1939]. The lower part of the profile, up to the level of maximum ionization, can be derived in real-time form virtual height measurements using an ionosonde [Stankov, 2012]. The virtual height of the ionosphere is measured by sending a pulsed radio wave vertically towards the ionosphere and receiving the reflection off the ionosphere. The delay of the signal is used to calculate the virtual height of the ionosphere, which is frequency dependent. An example ionogram is shown in Figure 4.2.

The ionospheric is birefringent: Appleton [1933] showed by experiment that under the influence of the earth' magnetic field the incoming electromagnetic wave is split into two characteristic waves at the base of the ionosphere.

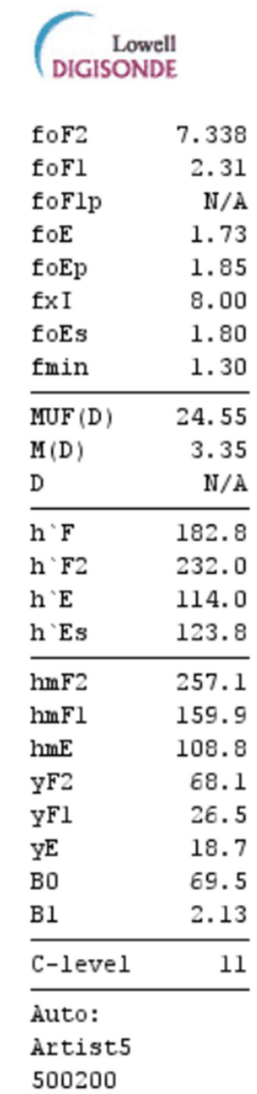

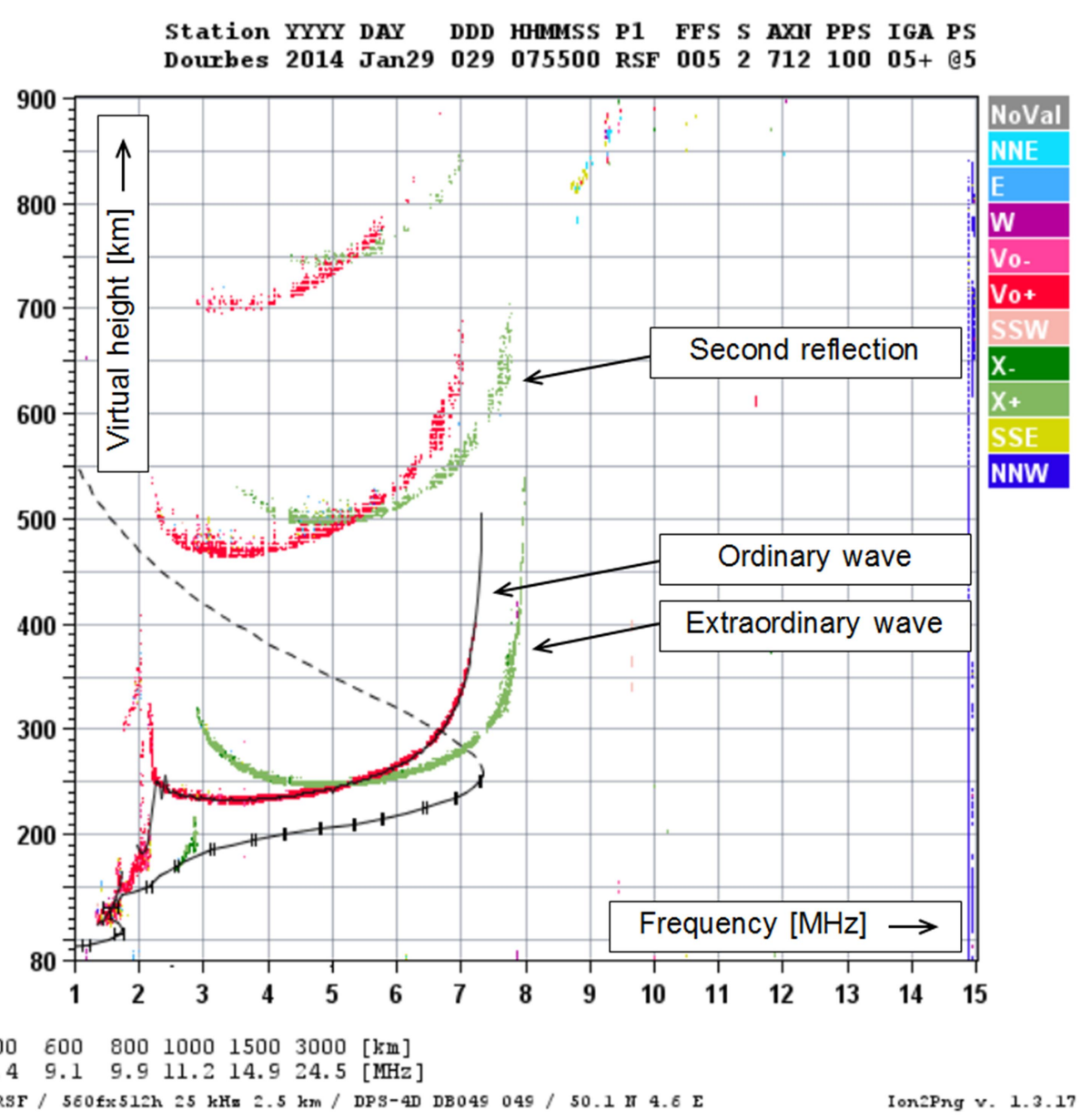

Figure 4.2 lonogram of Dourbes ionosonde (courtesy of Royal Observatory of Belgium) showing the virtual ionospheric height versus frequency on 29 Jan 2014, 0755 Coordinated Universal Time (UTC). Red trace represents the ordinary wave, green trace represents the extraordinary wave. Boxed texts are added by the authors. 
These characteristic waves, the ordinary and extraordinary wave, have circular polarization of opposite sense. They follow a different path through the ionosphere and experience different attenuation and show different behavior [Walden, 2009]. They therefore produce two slightly different traces in the ionogram of Figure 4.2, shown as green and red traces. Multiple reflections between the ionosphere and the ground may cause a secondary set of traces, but the main information is derived from the lower set of traces.

\subsubsection{Frequency dependency of ionospheric propagation}

Local maxima in the electron density profile at specific heights reflect radio waves, depending on the frequency used. These ionospheric regions are referred to as ionospheric 'layers'. Each layer has his own characteristics and is indicated with a letter (D, E and F) starting from the ground upwards. The reflection of radio waves against the E- and F-layer is responsible for most ionospheric radio wave propagation. The D-layer is present during daylight hours only and causes attenuation that is inversely proportional to the operating frequency [Webber, 1962]. The E-layer may contain local high density clouds, which are sparse both in occurrence and in localization, indicated as 'sporadic E' or 'Es'. During daytime the F-layer may split into two regions, a lower and less prominent F1-layer and a higher and denser F2-layer, to merge into one F-layer again at night. Diffuse irregularities in the topside ionosphere with high electron density are indicated as 'Spread- $F$ ' [Ossakow, 1981].

The highest frequency at which an electromagnetic wave will be reflected by an ionospheric layer when launched vertically, is called its 'plasma frequency' or 'critical frequency'. The critical frequency for the ordinary wave is indicated with ' $f o^{\prime}$ ' followed by the letter representing the layer, e.g. foE, foF1, foF2. Similarly, the critical frequency of the extraordinary wave is indicated with ' $f x$ ', e.g. fxF2. The critical frequency of the extraordinary wave is slightly higher than that of the ordinary wave, the difference being half the electron gyro frequency. The key parameters of the different layers are shown at the left side of the ionogram of Figure 4.2.

Only electromagnetic waves within a certain frequency range are reflected by the ionosphere. When the frequency is too low, the D-layer absorption may become prohibitive. When the frequency is above the critical frequency of the F-layer, radio waves that are launched vertically pass through the ionosphere and are lost in space. Waves that are launched at lower elevation angles travel a longer trajectory through the ionosphere and are be reflected back to earth still. The relationship between the elevation angle and the maximum frequency at which ionospheric radio wave propagation is supported was first formulated by Martyn [1935] and is known a as the 'Secant Law':

$$
\mathrm{MUF}=\mathrm{f}_{\mathrm{v}} \sec \theta
$$


where MUF is the (instantaneous) Maximum Usable Frequency, $\theta$ is the angle of incidence, and $f_{v}$ is the equivalent vertical frequency [Davies, 1990, pp. 157-158], the highest frequency that is reflected from the ionosphere when launched vertically. This formula, derived for a plane ionosphere, was later corrected for curved earth and ionosphere by [Smith, 1939]. The correction factor is small, between 1 and 1.1 typically. As can be seen from this formula, radio waves may be reflected back to earth at a considerable distance, while the area closer to the transmitter, requiring steeper elevation angles, is not covered. This creates an effect typical of ionospheric radio wave propagation, the so-called 'Skip Zone': no signals are received in a ring-shaped area around the transmitter, while outside that ring normal coverage occurs. This is illustrated in Figure 4.3.

In NVIS we want to cover a continuous area directly around the transmitter. To cover very short distances via the ionosphere we need to launch radio waves nearly vertically, therefore only frequencies below the critical frequency of the intended ionospheric layer can be used. At mid-latitudes in the northern hemisphere good NVIS frequencies typically range from 3 to $10 \mathrm{MHz}$.

\subsubsection{Variability of ionospheric propagation}

Ionospheric radio wave propagation is highly variable. The electron density profile of the ionosphere - and with it the ionospheric radio wave propagation - differs from location to location and varies with the earth magnetic field, the time of day and the season. On a longer timescale, it follows the 11-year solar activity cycle, the 'sunspot cycle', which is shown in Figure 4.4. Due to the complex and multivariate processes involved, ionospheric radio wave propagation can only be predicted statistically. But as will be shown in the Section 4.3, a set of specific characteristics for NVIS propagation can still be derived, and used for NVIS antenna design.

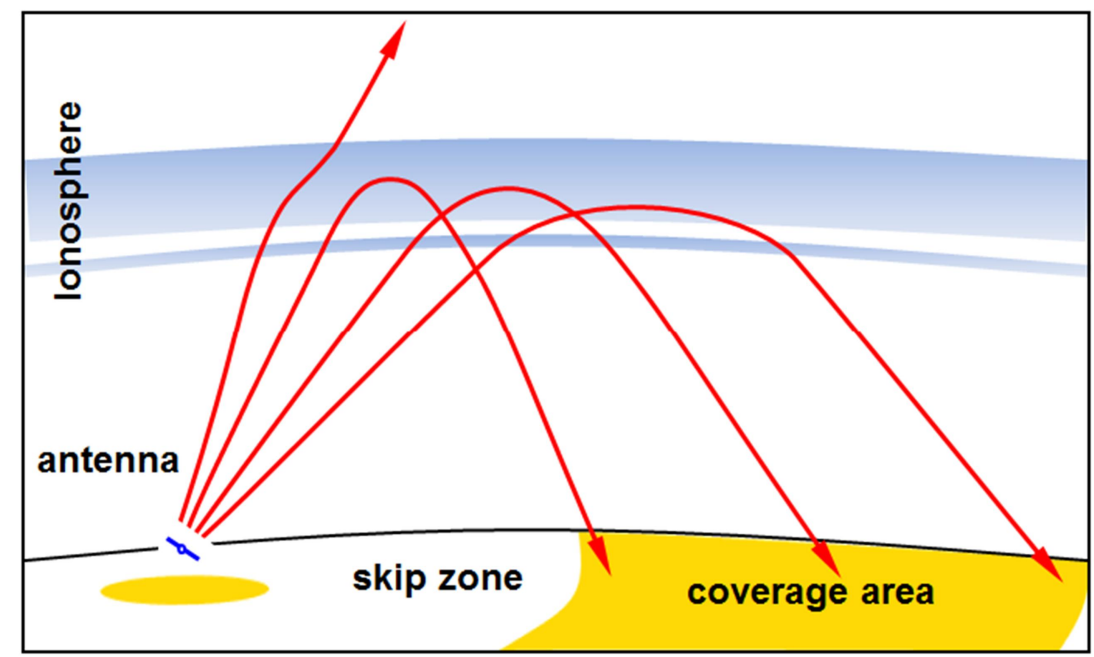

Figure 4.3 Ionospheric radio wave propagation above the critical frequency results in a skip zone. 


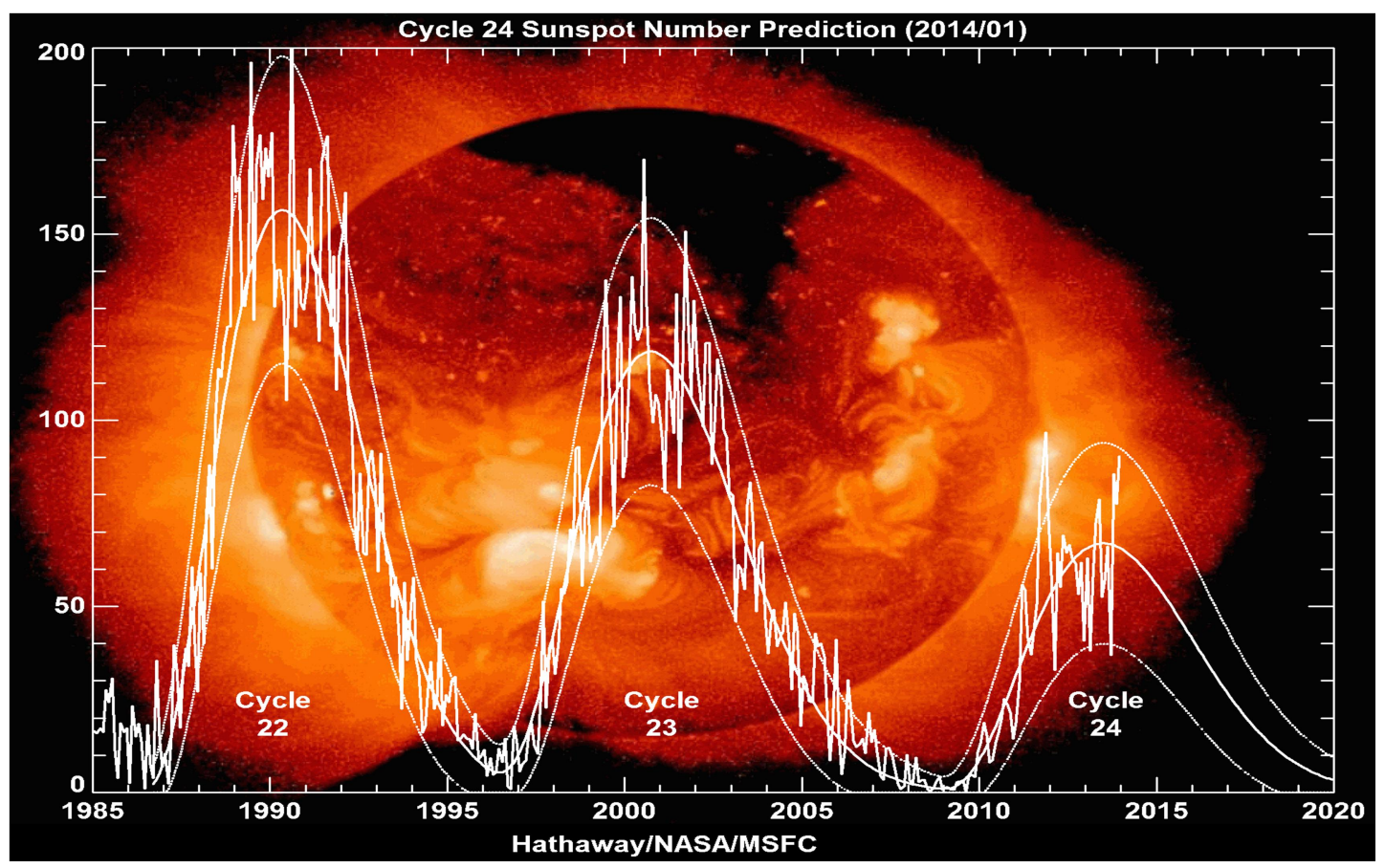

Figure 4.4 Variation of solar activity over three subsequent sunspot cycles. Vertical axis shows the Smoothed Sunspot Number (courtesy: Hathaway/NASA/MSFC).

Short term variability of ionospheric propagation - in terms of minutes, seconds and milliseconds - makes antenna comparison and hence the verification of a successful antenna optimization by experiment difficult. A solution to this problem will be proposed and demonstrated in Section 4.5. The influence of the long term variations on the experiments that are part of our research will be discussed in Section 4.6.

\subsection{NVIS ELEVATION ANGLES}

To cover very short distances via the ionosphere, radio waves must be launched nearly vertically. For continuous coverage of an area around the transmitter, radio waves must be launched from a certain angle upwards, depending on the size of the desired coverage area. Knowledge about these elevation angles is necessary for NVIS antenna optimization.

\subsubsection{NVIS elevation angle simulations.}

To establish the relationship between the radius of NVIS coverage area and the minimum elevation angle, a large number of simulations were performed using Proplab-Pro version 3, an ionospheric ray tracing program [Solar Terrestrial Dispatch, 2010]. As the virtual reflection height of the radio waves is frequency dependent, so is the elevation angle. Therefore several lines are shown in Figure 4.5, each representing the relationship between distance and elevation angle for a specified frequency. The line colors show the ionospheric layers involved. Red lines show the relationship between elevation angle and distance for frequencies below the critical frequency of the E-layer. The radio wave is reflected by the 
layer regardless of the elevation angle. There is a slight increase in reflection height with increasing frequency, but this does not influence the elevation angle significantly. The blue lines indicate that the frequency of the radio wave is above the critical frequency of the Elayer. Low elevation angles are still reflected by the layer, but higher angle pass through it. The waves that pass through are slightly refracted by that passage, after which they are reflected by the F-layer. The green lines show waves with sufficiently high frequency to pass through the E-layer unaltered, to reflect against the F-layer regardless of the elevation angle. At still higher frequencies the waves are only reflected when launched at low angles.

Two scenarios were examined. One represents a sunspot cycle minimum and is shown in Figure 4.5 , the other represents the maximum of a moderate sunspot cycle (similar to Cycle 23 in Figure 4.4) and is shown in Figure 4.6. The simulations use the International Reference lonosphere model version 2007 with the international electron density model of the Committee Consultative on Radiocommunication, the International Geomagnetic Reference Field magnetic field model, and the NeQuick topside model. IG Index (Effective Smoothed Sunspot Number) of 10 and $120, \mathrm{Ap}$ and $\mathrm{Kp}$ are set at 0 . Transmitter location was $52^{\circ} \mathrm{N}, 6^{\circ} \mathrm{E}$, simulation date was set to 10 November 2001, 10:30 UTC. Both simulations use the same date to accentuate the influence of the solar activity alone. Related signal strength levels and absorption are ignored, only the elevation angles for frequencies supported by the ionosphere are examined.

Figure 4.7 shows the ray paths for a fixed distance at increasing frequencies. The same color coding is used to indicate E-layer reflection (red), E-layer refraction followed by F-layer reflection (blue) and F-layer reflection (green). This figure illustrates that the elevation angle depends on the wanted skip distance, but also on the operating frequency. Single line graphs, thus ignoring the frequency dependency, for the relationship between elevation angle and skip distance can be found in [Davies, 1990, p. 139]. His curves for E-layer and Flayer reflections, correspond with our simulations, but only when frequencies near the MUF are assumed. Transmitter location is $52^{\circ} \mathrm{N}, 6^{\circ} \mathrm{E}$, with a path length of $150 \mathrm{~km}$, in the south direction. IG Index is 120, Ap and Kp are set at 0, simulation date is 10 Nov 2001 10:30 UTC.

These simulations illustrate that the NVIS elevation angle depends on coverage distance, but also on the operating frequency and the critical frequency of the $E$ and F-layers. Considering the variability of the ionosphere an all-inclusive relationship between elevation angle and distance cannot be given. However, when we choose an operating frequency that favors Flayer reflection and for a transmitter location at mid-latitudes we can still draw some important conclusions. To realize an NVIS coverage area with a radius of $150 \mathrm{~km}$ - the size of Switzerland or the State of Louisiana - elevation angles from $68^{\circ}$ to $90^{\circ}$ for low sunspots numbers or from $65^{\circ}$ to $90^{\circ}$ for high sunspot numbers seem to be a valid assumption. 


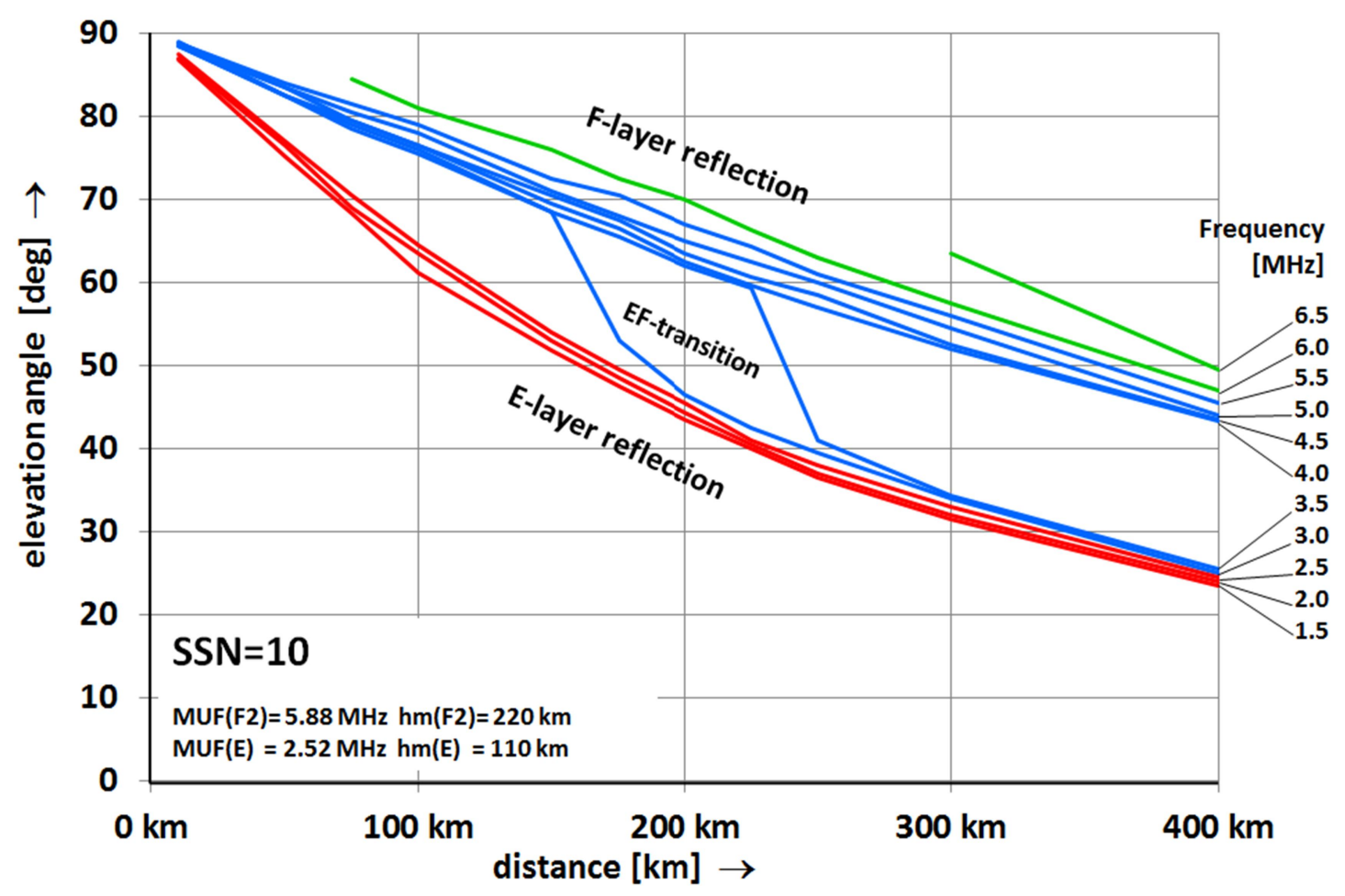

Figure 4.5 Relationship between elevation angle and distance for several frequencies. Simulations for the ordinary wave using Proplab Pro version 3 for IG Index (Effective Smoothed Sunspot Number) is 10, Ap and Kp are set at 0 . These values represent a solar cycle low.

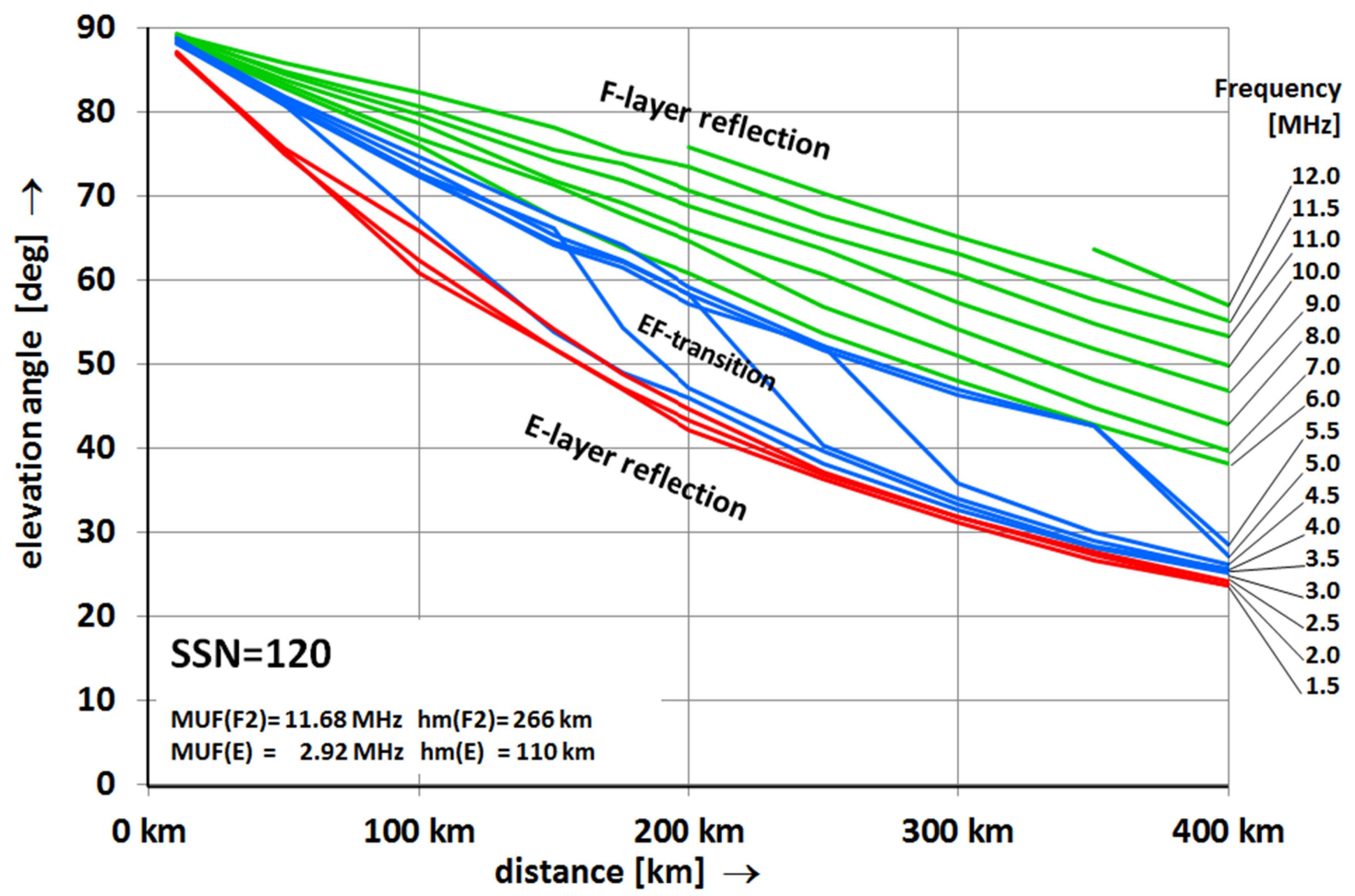

Figure 4.6 Relationship between elevation angle and distance for several frequencies. Simulations for the ordinary wave using Proplab-Pro version 3 for IG Index (Effective Smoothed Sunspot Number) is 120, Ap and Kp are set at 0 . These values represent a moderate solar cycle maximum. 


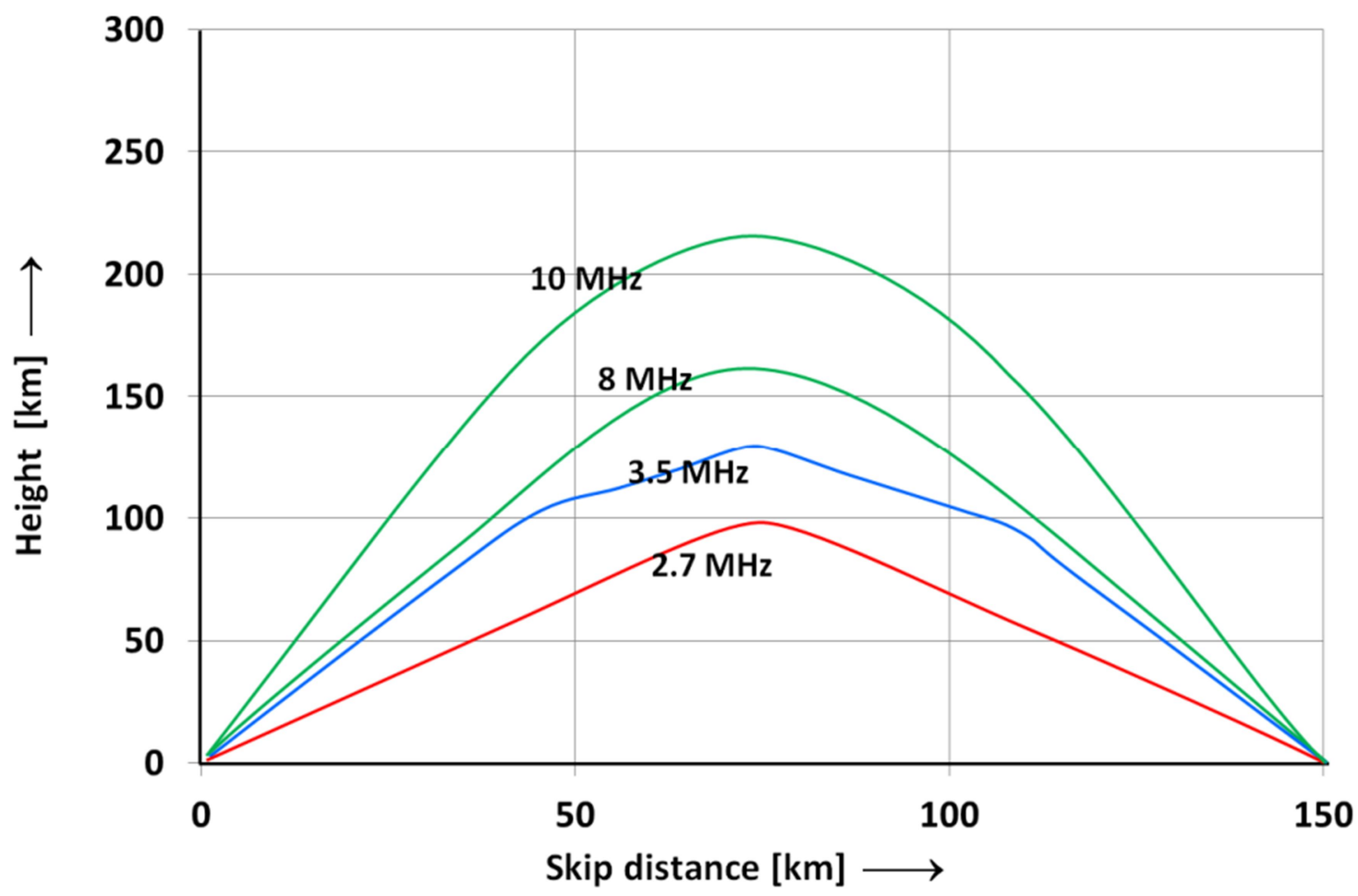

Figure 4.7 Ionospheric path and corresponding elevation angle for a fixed skip distance for 4 different frequencies. Proplab-Pro version 3 2-D ray tracing was used to create this example and only the ordinary wave paths are shown.

\subsubsection{NVIS elevation angle measurements}

To relate these theoretical findings with practical NVIS propagation properties, elevation angles of 85 NVIS stations were measured on $3.5 \mathrm{MHz}$ and $7 \mathrm{MHz}$, during a national amateur radio contest. The results were originally published by the authors in amateur radio magazines such as [Witvliet, 2005]. The measurements were performed on 10 and 11 November 2001, between 08:00 and 11:00 UTC. Over 300 measurements were made, recording azimuth angle and elevation angle. A professional Radio Direction Finder (RDF) type Rohde \& Schwarz (R\&S) DDFOxM was used, located $52.24^{\circ} \mathrm{N}, 5.08^{\circ} \mathrm{E}$. The RDF consists of 9 crossed loop antennas placed in a 50 meter circle, connected to digital receivers followed by correlators. The crossed loops antennas are fed using a phasing network to provide circular polarization with selectable direction of rotation. During the measurements, the polarization that yielded highest reliability, as indicated by the RDF, was selected. A picture of three of these crossed loop antennas is shown in Figure 4.8.

The NVIS stations were spread across the country, at distances ranging from 9 to $165 \mathrm{~km}$. Most NVIS stations used 100 Watts transmitters and single-wire, horizontally polarized antennas. Figure 4.9 shows the location of these NVIS stations as red dots on the map of The Netherlands. Thin red lines show the azimuth angle and distance from the RDF to each NVIS station. Equidistant circles with $50 \mathrm{~km}$ increments are superimposed in grey. 


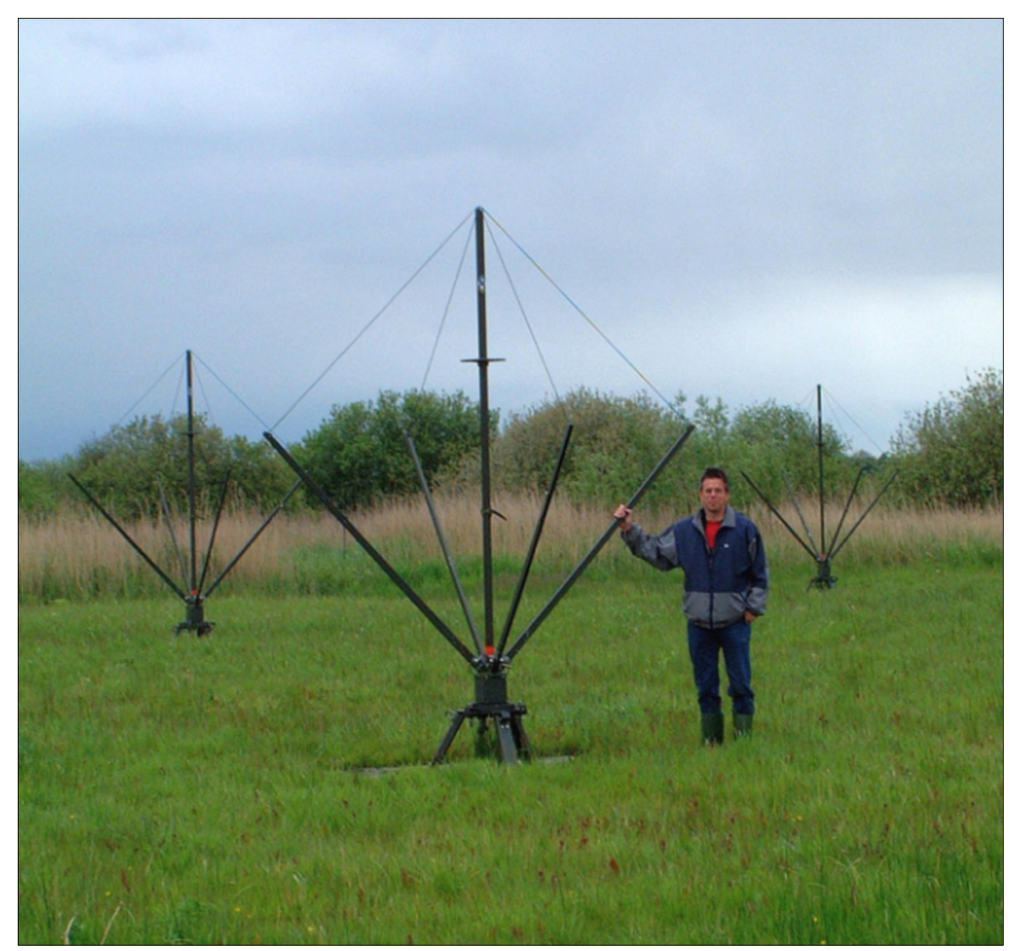

Figure 4.8 Three of the nine crossed loop antennas of the Rohde \& Schwarz DDF0xM Radio Direction Finder.

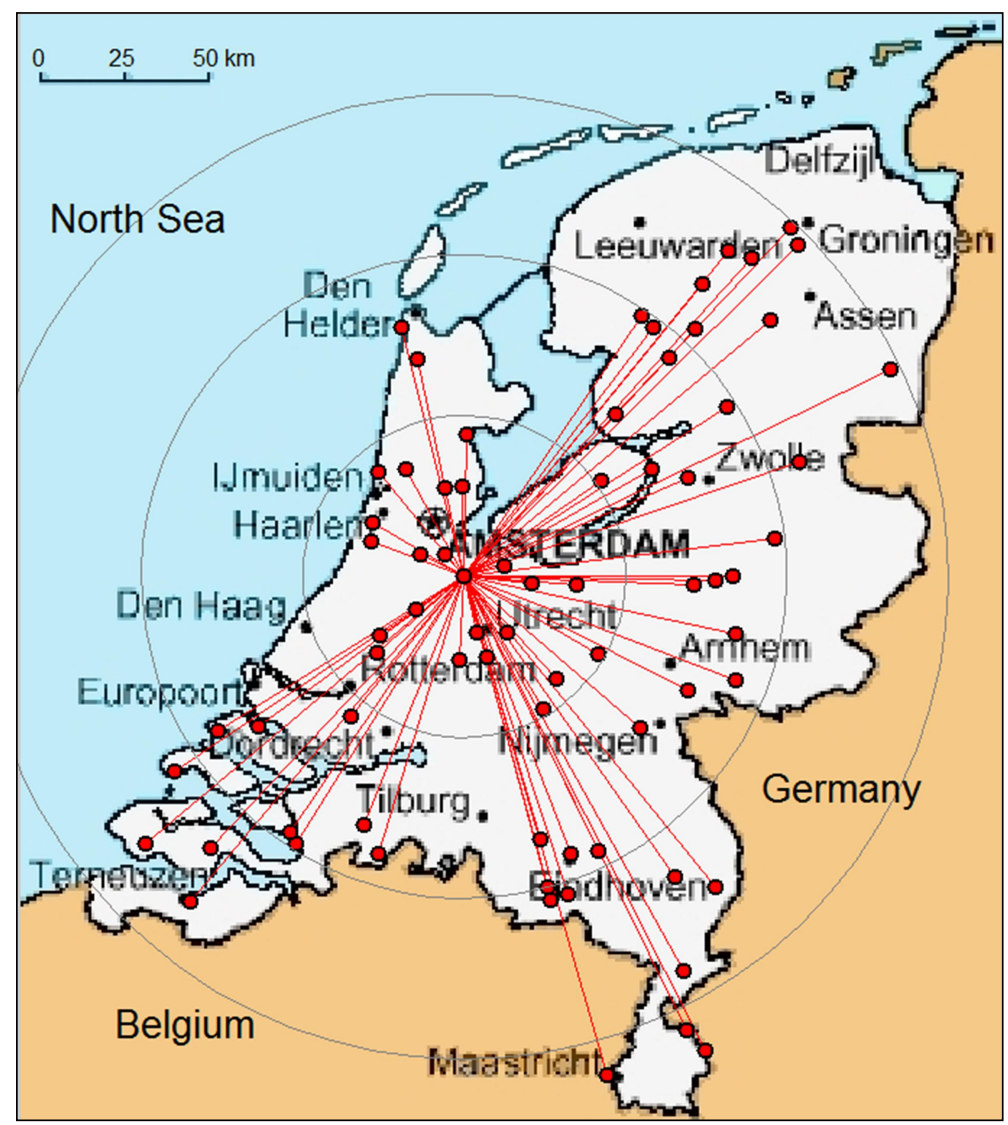

Figure 4.9 Azimuth and distance from the Radio Direction Finder to the 85 NVIS stations. 
For verification purposes, each radio station was identified by its call sign. Using the address information registered with the call sign, the measured azimuth angle was compared with the expected direction. Where this azimuth angle had a deviation greater than $15^{\circ}$, the station owner was contacted to verify the transmitter location. In most cases a temporary location was used which provided a good match with the measured azimuth and the correct distance was recorded for each measurement. Figures 4.10 and 4.11 show the distribution of the measured elevation angle for these 300 measurements on $3.5 \mathrm{MHz}$ and $7 \mathrm{MHz}$.

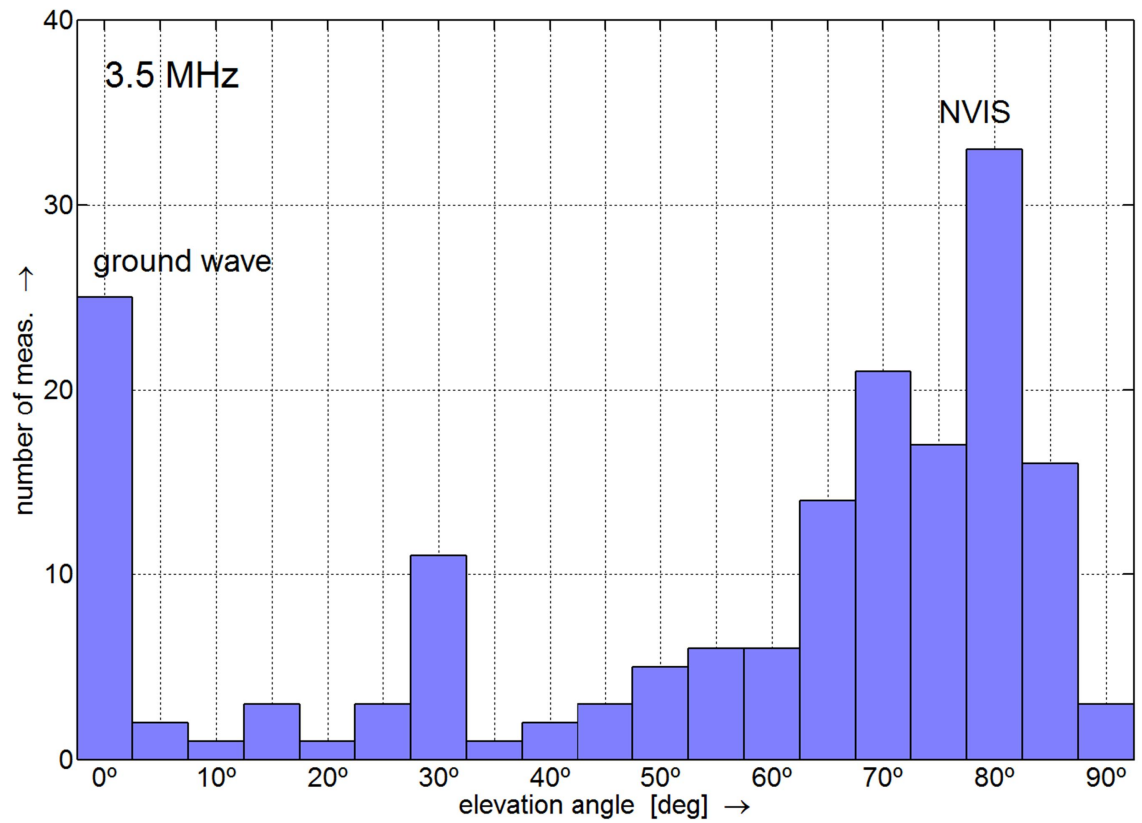

Figure 4.10 Histogram of measured elevation angles on $3.5 \mathrm{MHz}$.

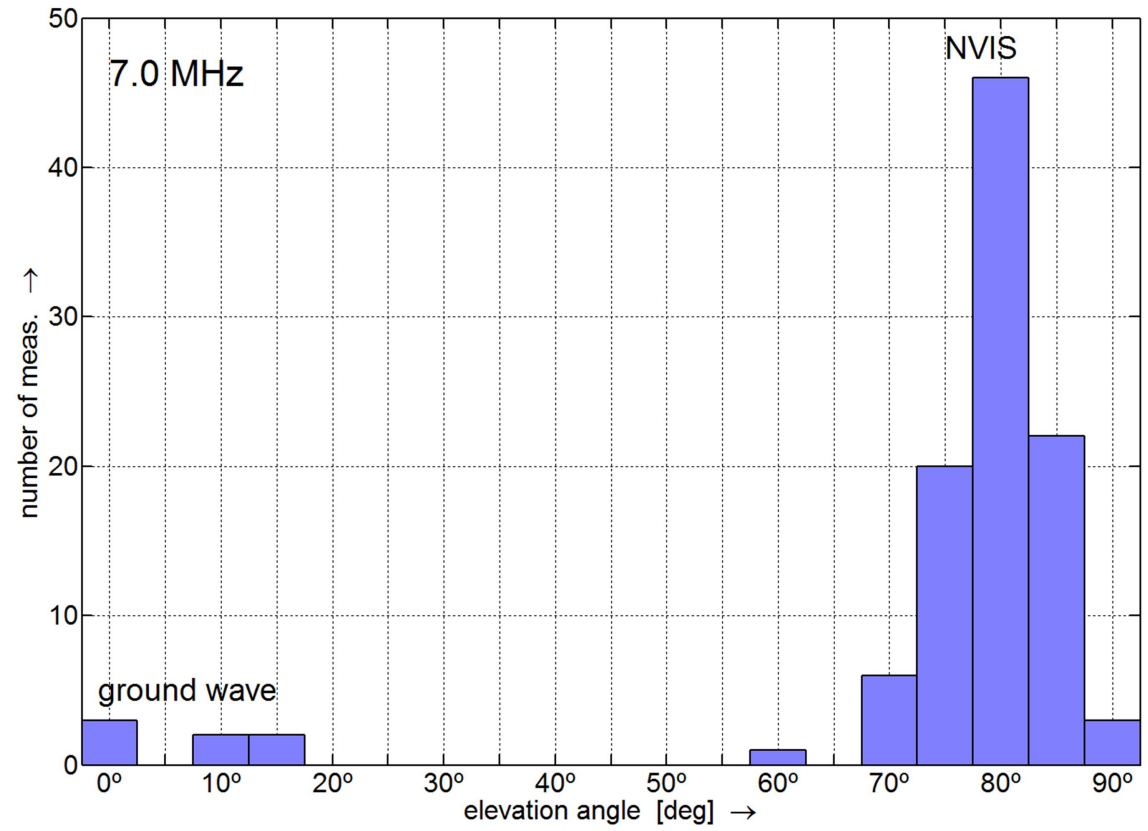

Figure 4.11 Histogram of measured elevation angles on $7.0 \mathrm{MHz}$. 
Measurements with approximately $0^{\circ}$ elevation angle indicate arrival via ground wave propagation, the elevation angles above $70^{\circ}$ are NVIS. The high proportion of high angle measurements shows the dominance of NVIS propagation over ground wave. This was very significant at $7.0 \mathrm{MHz}$, where even radio stations located just $20 \mathrm{~km}$ away could only be received via NVIS. According to theory, the ground wave reaches farther on lower frequencies, which explains the higher proportion of ground wave measurements on 3.5 $\mathrm{MHz}$. Figures 4.12 and 4.13 show the measured elevation angle as a function of the distance.

The measurements on $3.5 \mathrm{MHz}$ show a large spreading. This may have three reasons: (1) the accuracy of the RDF is lower on $3.5 \mathrm{MHz}$ as its physical dimensions are smaller compared with the wavelength, (2) and the RDF may to have more difficulty in resolving the mix of ground wave and skywave components at short distances, and (3) due to the lower frequency both E-layer and F-layer reflections may have occurred within the 3 hour measurement interval. As these graphs show, NVIS is dominant over ground wave propagation at distances greater than approximately $40 \mathrm{~km}$ on $3.5 \mathrm{MHz}$, and greater than 20 $\mathrm{km}$ on $7 \mathrm{MHz}$. The measured elevation angles for NVIS coverage from 0 to $165 \mathrm{~km}$ range from $65-90^{\circ}$ on $3.5 \mathrm{MHz}$, and from $70-90^{\circ}$ on $7 \mathrm{MHz}$.

\subsection{NVIS ANTENNA OPTIMIZATION}

The properties of the employed transmit and receive antennas must match the intended propagation mechanism and suppress unwanted propagation. For NVIS this means that an antenna must be selected with a vertical radiation pattern favoring the high elevation angles found through simulation and measurement in Section 4.3, while suppressing radiation at lower elevation angles. Matching the polarization of the transmit and receive antennas to the propagation mechanism is not considered in this chapter, but will be discussed in Chapter 6. A wide range of antennas types is available for short wave applications, each with its specific properties concerning radiation pattern, gain, efficiency, gain bandwidth, impedance bandwidth and polarization.

However, due to the long wavelength, antennas for the intended frequency range will be large. For mobile applications small loop antennas are popular. For ad-hoc field operations larger wire antennas strung between existing structures or portable masts provide higher antenna gain and more bandwidth. Also arrays can be formed of multiple identical antenna elements to produce an enhanced radiation pattern [Kraus, 1988, pp. 127-130]. Antenna arrays for transmission are large and require multiple supports, complex power splitting networks and phase lines. Receive antenna arrays, on the other hand, may be composed of a number of small, low weight active antennas with much simpler low power splitters and phasing harness. Such an antenna array can be deployed quickly for base stations in ad-hoc operations. Modern high-end HF radio transceivers supporting the use of separate transmit and receive antennas could be used in emergency base stations. 


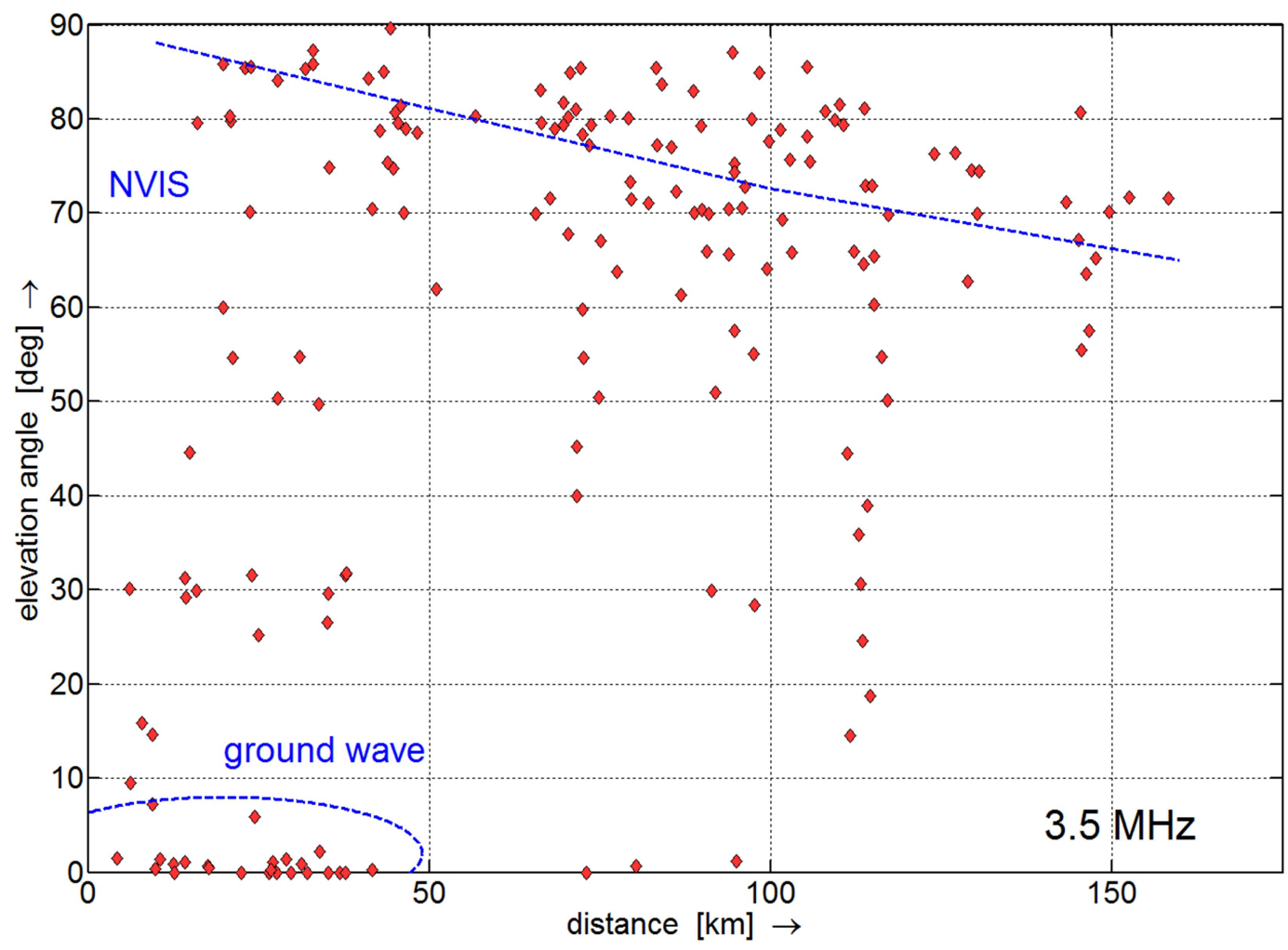

Figure 4.12 Measured elevation angle versus distance on $3.5 \mathrm{MHz}$. The blue dashed line shows the expected value, taken from Figure 4.5.

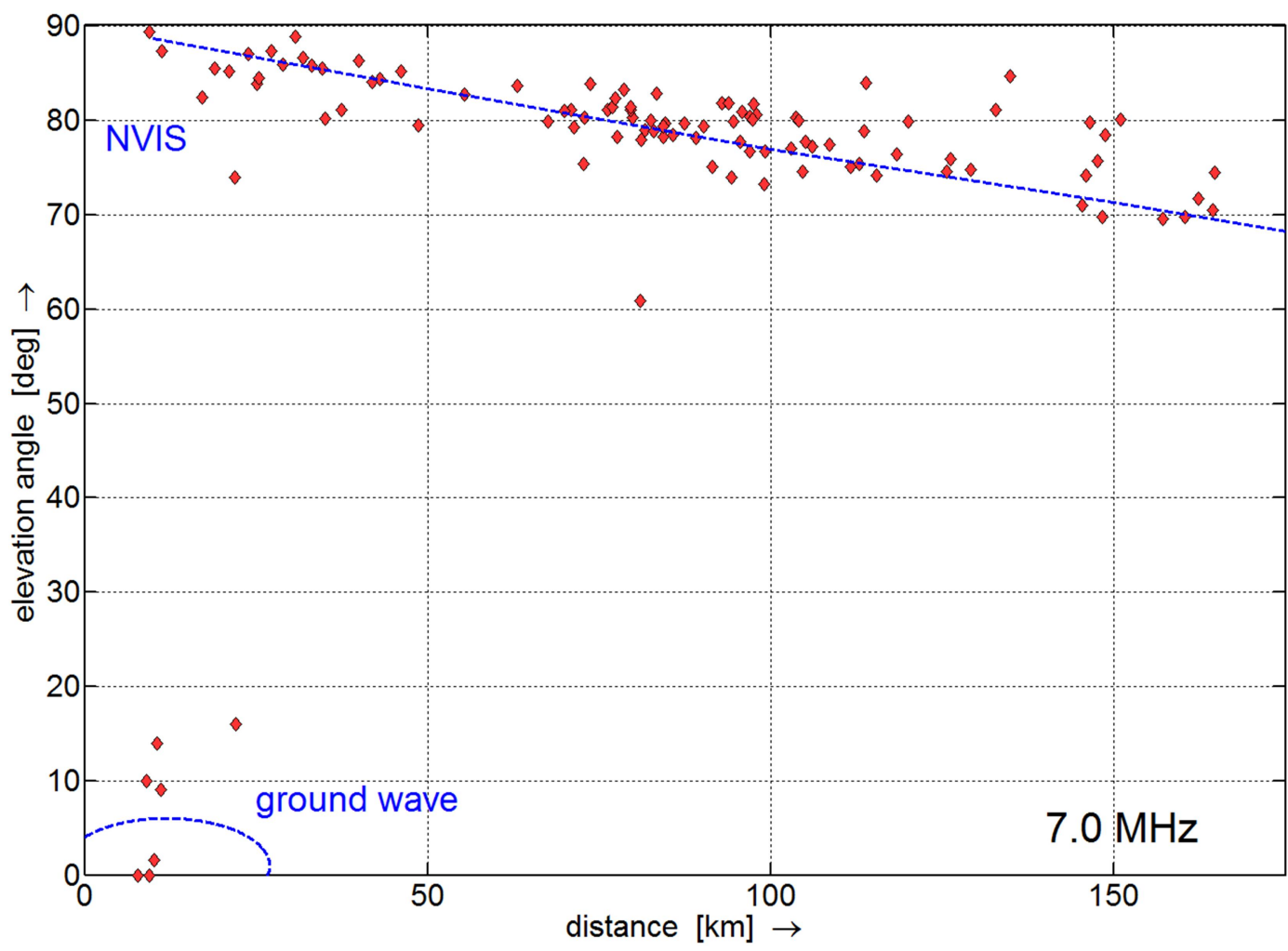

Figure 4.13 Measured elevation angle versus distance on 7.0 MHz. The blue dashed line shows the expected value, taken from Figure 4.5. 
At mid-latitudes, when a receive antenna with circular polarization is used, the selection of the ordinary or the extraordinary wave may reduce dispersion and fading. Simultaneous reception of left-hand and right-hand circular polarization can be used for diversity reception. Circular polarization for NVIS can be achieved with two perpendicular horizontally polarized (dipole) antennas fed with $90^{\circ}$ phase difference as will be shown in Section 6.3 and paragraph 7.3.2. As this antenna requires only one support, it may be practical even in temporary or ad-hoc installations.

\subsubsection{Influence of antenna height}

The NVIS propagation mechanism restricts the use of frequencies in the range of approximately 3 to $10 \mathrm{MHz}$, corresponding to wavelengths of 30 to 100 meters. NVIS antennas are therefore large and often basic antenna types realized as wire antennas strung at low heights - in terms of wavelengths - above ground. As a consequence, NVIS antenna designers must consider the influence of ground proximity: ground absorption, and beam forming due to ground reflection. To analyze the effect of antenna height on ground losses and ground reflection gain, a large number of half-wave wire dipoles was modeled at different heights and above different soil types on 5.39 MHz. Numerical Electromagnetics Code (NEC) 4.1 was used, a Method-of-Moments antenna simulation software created at Lawrence Livermore National Laboratories [Burke, 2004]. It includes a Sommerfeld-Norton ground model for realistic simulation of ground reflection and ground loss [Burke, 1981]. A wire radius of $1 \mathrm{~mm}$ was assumed. Both the wire radius and the ground proximity influence the resonant length of the antenna. Therefore, in each simulation, the antenna length was corrected to achieve resonance. A selection of the simulations is shown in Figure 4.14, illustrating the influence of increasing antenna height on the antenna gain and the vertical radiation pattern of horizontal half-wave dipoles above farmland soil ( $\sigma \approx 20 \mathrm{mS} / \mathrm{m}, \varepsilon_{\mathrm{r}} \approx 17$ ).

When the antenna is mounted at a very low height $(0.02 \lambda)$ the antenna gain is low. The antenna diagram shows considerable directivity, but a substantial portion of the transmit power is lost in the ground underneath the antenna. With increasing antenna height $(0.06 \lambda)$ the amount of beam forming due to ground reflection decreases slowly, and the antenna directivity decreases. However, the ground losses decrease much faster, so that the resulting antenna gain increases, until maximum antenna gain is realized around $0.2 \lambda$. When the antenna height is further increased (in our example to $0.4 \lambda$ ) the radiation pattern flattens and the maximum antenna gain occurs at lower elevation angles. At the elevation angles needed for NVIS, however, the antenna gain decreases. This process continues until at $0.5 \lambda$ a minimum is found at $90^{\circ}$ elevation angle. At heights above $0.5 \lambda$ the high angle radiation starts to increase again, but now side lobes at lower elevation angles are created, which we consider undesirable because of the increasing interference to and from other stations located farther away. 


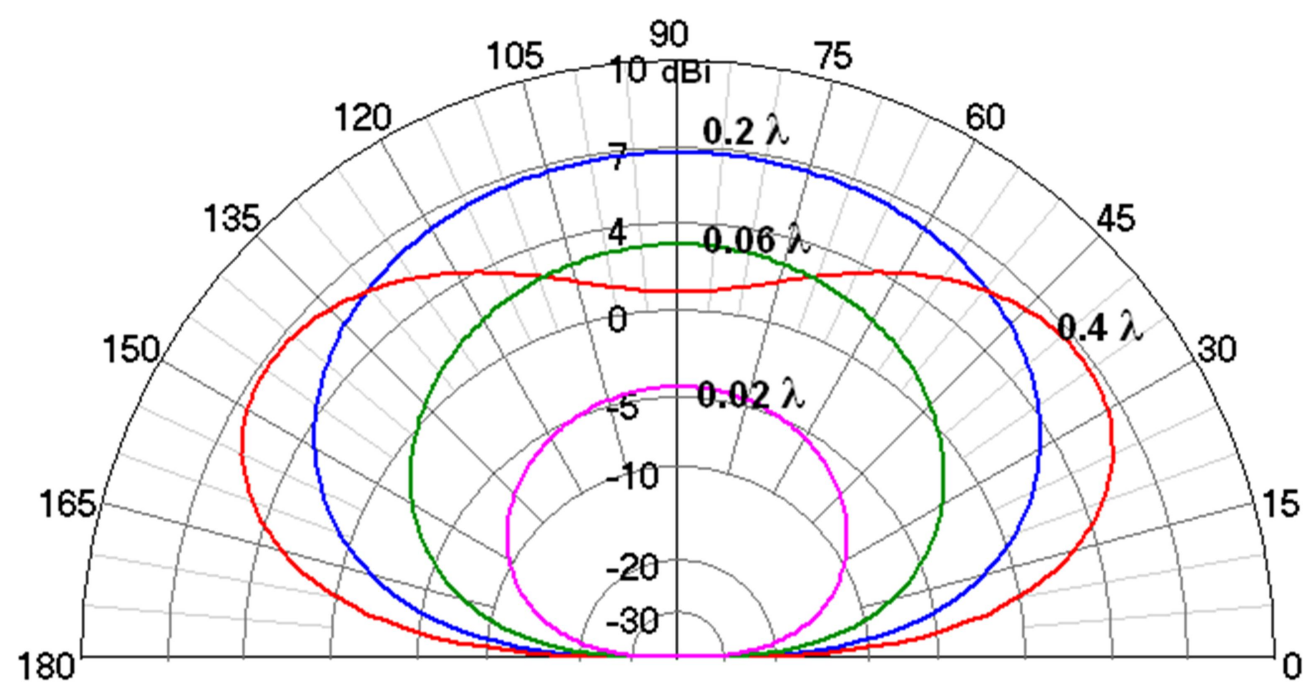

Figure 4.14 Vertical radiation pattern of a horizontal half-wave dipole antenna $0.02 \lambda, 0.06 \lambda, 0.2 \lambda$, and $0.4 \lambda$ above farmland soil on $5.39 \mathrm{MHz}$. Intensity axis shows antenna gain in decibels over an isotropic radiator (dBi).

\subsubsection{Simulated optimum NVIS transmit antenna height}

Normally, antenna gain is defined in the direction of maximum radiation. This definition cannot be used in NVIS research, as Figure 4.14 illustrates: with the antenna mounted at $0.4 \lambda$ above ground the maximum gain occurs at an elevation angle of $35^{\circ}$, while the antenna gain at NVIS elevation angles is much lower. To produce the highest field strength in the coverage area, the radiated power has to be directed towards the high elevation angles used in NVIS. Therefore, to be used in our optimization, we introduce 'NVIS Antenna Gain' $\left(G_{N V I S}\right)$, as the average antenna gain at NVIS elevation angles, i.e. between $70^{\circ}$ and $90^{\circ}$ for a coverage area with a radius of $150 \mathrm{~km}$. Another elevation angle range can be chosen if a larger NVIS coverage area is targeted.

$$
\mathrm{G}_{\mathrm{NVIS}}=\frac{\int_{\varphi=0}^{2 \pi} \int_{\theta=0}^{\frac{\pi}{9}} \mathrm{G}(\theta, \varphi) \sin \theta \mathrm{d} \theta \mathrm{d} \varphi}{\int_{\varphi=0}^{2 \pi} \int_{\theta=0}^{\frac{\pi}{9}} \sin \theta \mathrm{d} \theta \mathrm{d} \varphi}=\frac{\int_{\varphi=0}^{2 \pi} \int_{\theta=0}^{\frac{\pi}{9}} \mathrm{G}(\theta, \varphi) \sin \theta \mathrm{d} \theta \mathrm{d} \varphi}{2 \pi\left(1-\cos \frac{\pi}{9}\right)}
$$

where $\varphi$ is the azimuth angle and $\theta$ is the zenith angle, both expressed in radians, and $G(\theta$, $\varphi)$ the antenna gain in the direction $(\theta, \varphi)$, expressed as a linear value. Elevation angles of $70^{\circ}$ to $90^{\circ}$ correspond with zenith angles of $0^{\circ}$ to $20^{\circ}$ or 0 to $\pi / 9$ radians. 
In analogy, 'NVIS Directivity' $\left(D_{\text {NVIS }}\right)$ is defined as the average directivity for elevation angles between $70^{\circ}$ and $90^{\circ}$ :

$$
\mathrm{D}_{\mathrm{NVIS}}=\frac{\int_{\varphi=0}^{2 \pi} \int_{\theta=0}^{\frac{\pi}{9}} \mathrm{D}(\theta, \varphi) \sin \theta \mathrm{d} \theta \mathrm{d} \varphi}{2 \pi\left(1-\cos \frac{\pi}{9}\right)}
$$

where $D(\theta, \varphi)$ is the directivity the direction $(\theta, \varphi)$, i.e. the antenna gain in that direction divided by the average antenna gain over all possible spatial angles, expressed as a linear value. The output of the NEC 4.1 simulations is now reprocessed using (2) and (3). Figure 4.15 shows $G_{N V I S}$ and $D_{\text {NVIS }}$ as a function of the antenna height for farmland soil. It can be seen that for farmland the NVIS Directivity varies only slowly with height with an optimum $h_{R X}$ at $0.09 \lambda$, whereas the NVIS Antenna Gain has a distinct optimum $h_{T X}$ at $0.19 \lambda$ and sharply decreasing at low heights due to excessive ground loss. The NVIS Antenna Gain is $11.3 \mathrm{~dB}$ lower at $0.02 \lambda$.

Figure 4.16 compares the NVIS Antenna Gain for several ground types. The optimum NVIS transmit antenna height lies between $0.18 \lambda$ and $0.22 \lambda$ for most ground types. Above sea water the optimum height is $0.13 \lambda$. Higher ground conductivity and higher permittivity result in higher NVIS Antenna Gain, with $2.2 \mathrm{~dB}$ increase from urban soil to clay soil, and another $1.1 \mathrm{~dB}$ from clay soil to sea water.

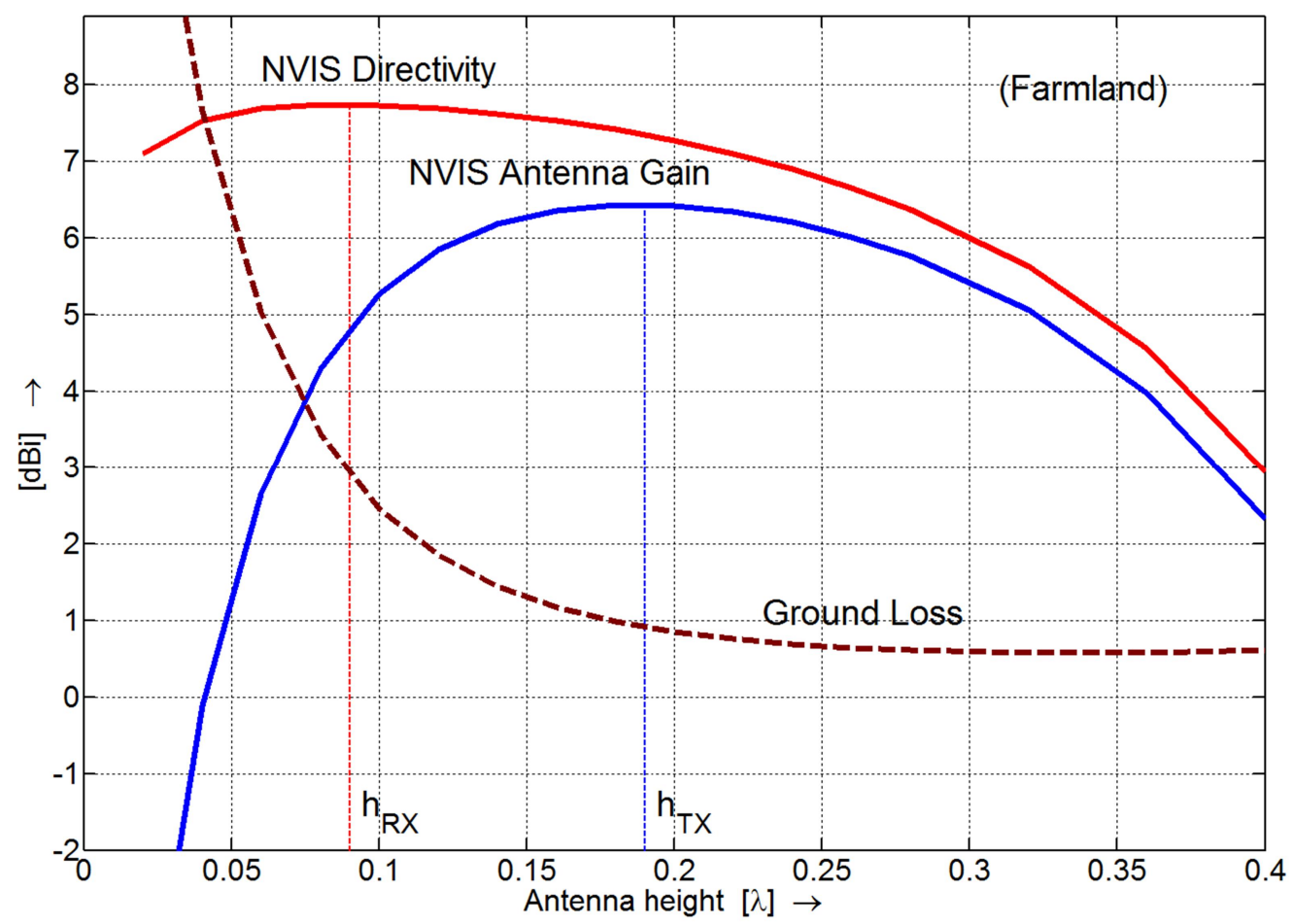

Figure 4.15 NVIS Antenna Gain (blue) and Directivity (red) of a horizontal half-wave dipole antenna versus height above farmland soil on 5.39 MHz. Ground loss in dB. 
The optimum NVIS Transmit Antenna Heights (hTX) found for several soil types are summarized in Table 4.1. For completeness it must be noted that some fresh water lakes show conductivities of up to $50 \mathrm{~ms} / \mathrm{m}$ due to (industrial) pollutants. This increases the antenna gain by 2-3 dB from the values found for fresh water lakes for an antenna height of $0.02 \lambda$. The effect is less for greater heights. In that case the optimum height will be close to that of clay ground.

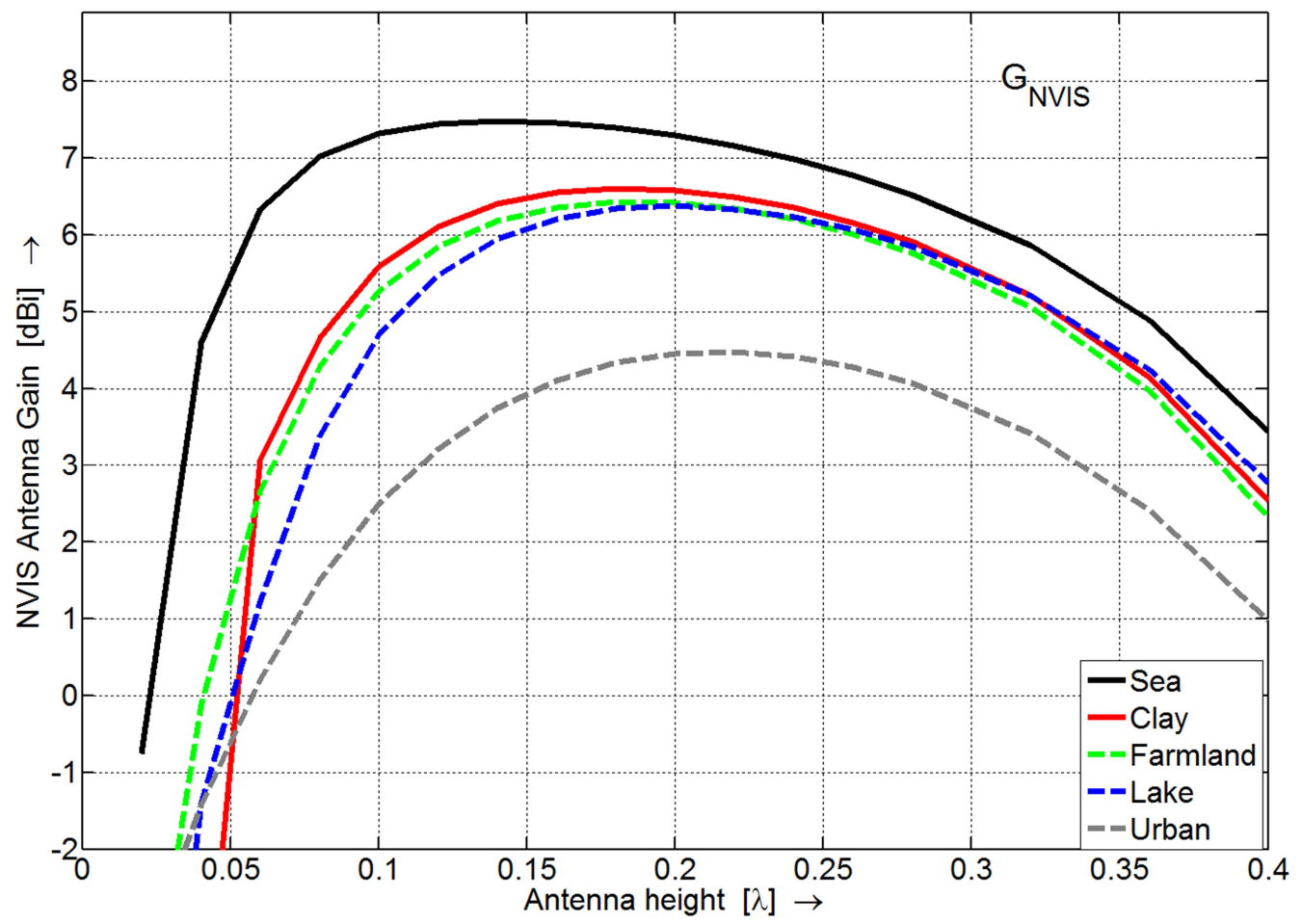

Figure 4.16 NVIS Antenna Gain ( $G_{\text {NVIS }}$ ) of a horizontal half-wave dipole antenna versus height for several soil types on 5.39 MHz. NVIS Antenna Gain is the average antenna gain for NVIS elevation angles, here between and for a coverage area with a radius of $150 \mathrm{~km}$.

Table 4.1 Optimum NVIS transmit antenna height $\left(\mathrm{h}_{\mathrm{TX}}\right)$ - the height above ground for a horizontal half-wave dipole antenna that yields the highest NVIS Gain - for several soil types.

\begin{tabular}{cccc}
\hline \hline Soil type & $\sigma$ & $\varepsilon_{\mathrm{r}}$ & $\mathrm{h}_{\mathrm{TX}}$ \\
\hline Urban & $1 \mathrm{mS} / \mathrm{m}$ & 5 & $0.22 \lambda$ \\
Lake* $^{*}$ & $5 \mathrm{mS} / \mathrm{m}$ & 80 & $0.20 \lambda$ \\
Farmland & $20 \mathrm{mS} / \mathrm{m}$ & 17 & $0.19 \lambda$ \\
Clay & $30 \mathrm{mS} / \mathrm{m}$ & 20 & $0.18 \lambda$ \\
Sea & $5000 \mathrm{mS} / \mathrm{m}$ & 80 & $0.13 \lambda$ \\
\hline \hline
\end{tabular}

* Some rivers and fresh water lakes show conductivities of $50 \mathrm{mS} / \mathrm{m}$ or more due to the presence of (industrial) pollutants. 


\subsubsection{Simulated optimum NVIS receive antenna height}

Optimization of the receive antenna height is similar to that of the transmit antenna, but not identical. On the receive side, the reception threshold is determined by signal-to-noise ratio (SNR), rather than signal strength [Kraus, 1988, p. 766]. So the antenna must be selected for the highest discrimination between NVIS signals and unwanted signals arriving from other directions, and for the lowest susceptibility to natural and man-made ambient noise, which may arrive via skywave or via line of sight. As the origin of the interference and the ambient noise is not known a priori, all azimuth and elevation angles are considered equally likely to produce interference and noise. Likewise, if the exact location of the NVIS signal source within the coverage area is not known, best approximation is to use the average antenna gain calculated over the corresponding elevation angles for the wanted signal. With these assumptions the Discrimination Factor (DF) is equal to the division of the average antenna gain over the NVIS elevation angles and the average gain over all possible elevation angles, and corresponds with the NVIS Directivity.

$$
\mathrm{DF}=\frac{\int_{\varphi=0}^{2 \pi} \int_{\theta=0}^{\frac{\pi}{9}} \mathrm{G}(\theta, \varphi) \sin \theta \mathrm{d} \theta \mathrm{d} \varphi / \int_{\varphi=0}^{2 \pi} \int_{\theta=0}^{\frac{\pi}{9}} \sin \theta \mathrm{d} \theta \mathrm{d} \varphi}{\int_{\varphi=0}^{2 \pi} \int_{\theta=0}^{\pi} \mathrm{G}(\theta, \varphi) \sin \theta \mathrm{d} \theta \mathrm{d} \varphi / \int_{\varphi=0}^{2 \pi} \int_{\theta=0}^{\frac{\pi}{9}} \sin \theta \mathrm{d} \theta \mathrm{d} \varphi}=\frac{\mathrm{G}_{\mathrm{NVIS}}}{\eta}=\mathrm{D}_{\mathrm{NVIS}}
$$

This implies that, on reception, NVIS Directivity must be optimized rather than NVIS Antenna Gain. The ground reflection still influences the vertical radiation pattern and contributes to NVIS Directivity, but lower antenna efficiency due to ground loss no longer plays a role, as both wanted and unwanted signals suffer the same loss. That is: as long as the receiver noise figure is low enough, so that the ambient noise determines the reception threshold. To calculate the theoretical maximum NVIS Directivity that can be realized, let us consider a perfect conical beam towards the zenith with $40^{\circ}$ beam width, with a uniform sensitivity over the elevation angles from $70^{\circ}$ to $90^{\circ}$ and no response at all at other elevation angles. The directivity of such an idealized antenna can be calculated as:

$$
\mathrm{D}_{\mathrm{NVIS}, \max }=\frac{4 \pi}{\int_{\varphi=0}^{2 \pi} \int_{\theta=0}^{\frac{\pi}{9}} \sin \theta \mathrm{d} \theta \mathrm{d} \varphi}=\frac{4 \pi}{2 \pi\left(1-\cos \frac{\pi}{9}\right)} \approx 33.16
$$

The maximum achievable $D_{\mathrm{NVIS}}$ is $10 \log _{10}(33.16)=15.2 \mathrm{dBi}$. If a uniform distribution of the ambient noise is assumed, the SNR of such an antenna would also be $15.2 \mathrm{~dB}$ higher, which is substantial. Most practical implementations will not achieve such values, although an array of active receive antennas employing digital beam forming may approach this value [Van Veen, 1988]. To analyze the influence of the receive antenna height, NVIS Directivity is 
plotted against antenna height for different ground types in Figure 4.17. The optimum NVIS Receive Antenna Heights $\left(h_{R X}\right)$ found for several soil types are summarized in Table 4.2. The receive antenna height seems not critical: the variation in NVIS Directivity is only $0.8 \mathrm{~dB}$ over a range from $0.02 \lambda$ to $0.22 \lambda$. Also the difference between the various soil types is small, less than $1 \mathrm{~dB}$.

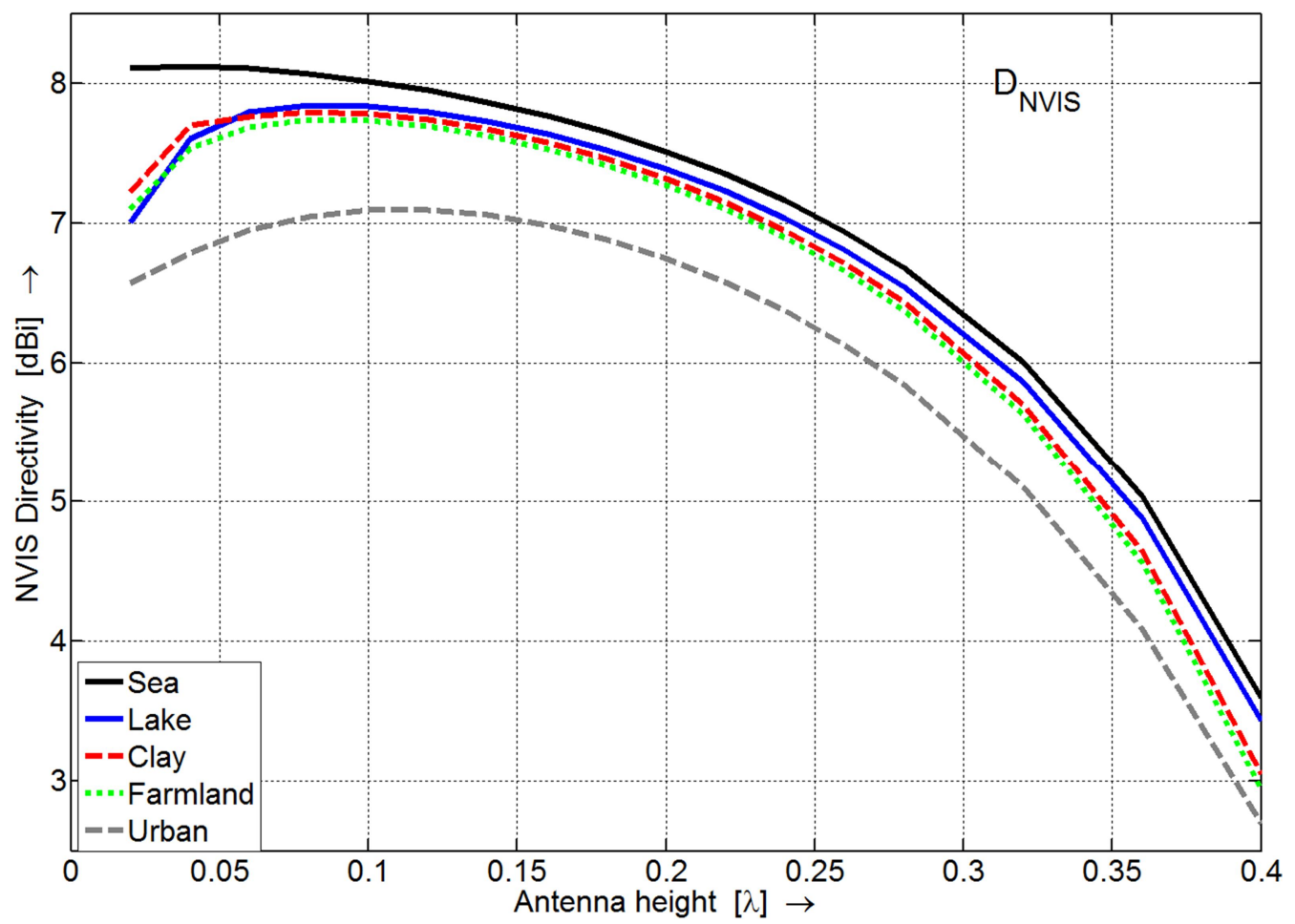

Figure 4.17 NVIS Directivity ( $D_{\text {NVIS }}$ ) of a horizontal half-wave dipole antenna versus height for several soil types on $5.39 \mathrm{MHz}$. NVIS Directivity is the average directivity for NVIS elevation angles, here between $70^{\circ}$ and $90^{\circ}$ for a coverage area with a radius of $150 \mathrm{~km}$.

Table 4.2 Optimum NVIS receive antenna height $\left(h_{R X}\right)$ - the height above ground for a horizontal half-wave dipole antenna that yields the highest NVIS Directivity - for several soil types.

\begin{tabular}{lrrl}
\hline \hline Soil type & $\sigma$ & $\varepsilon_{\mathrm{r}}$ & $\mathrm{h}_{\mathrm{RX}}$ \\
\hline Urban & $1 \mathrm{mS} / \mathrm{m}$ & 5 & $0.11 \lambda$ \\
Lake* & $5 \mathrm{mS} / \mathrm{m}$ & 80 & $0.09 \lambda$ \\
Farmland & $20 \mathrm{mS} / \mathrm{m}$ & 17 & $0.09 \lambda$ \\
Clay & $30 \mathrm{mS} / \mathrm{m}$ & 20 & $0.08 \lambda$ \\
Sea & $5000 \mathrm{mS} / \mathrm{m}$ & 80 & $0.04 \lambda$ \\
\hline \hline Some rivers and & fresh water lakes show \\
conductivities of $50 \mathrm{mS} / \mathrm{m}$ or more due to the \\
presence of (industrial) pollutants.
\end{tabular}




\subsection{COMPARISON OF HF ANTENNA PERFORMANCE IN THE PRESENCE OF FADING}

The optimum antenna heights are found through simulation and therefore require empirical verification. However, to obtain an accurate and reproducible antenna gain and antenna SNR comparison on HF is challenging. lonospheric radio wave propagation - including NVIS is subject to signal fading caused by changing properties of the ionosphere and by interference of waves traveling different paths through the ionosphere (multi-path fading).

During our observations, the signal experienced fast multi-path fading with an interval time of 2-6 seconds and notches varying in depth between 10 and $30 \mathrm{~dB}$, superimposed on slow fading over an interval of 15-60 seconds. The channel response - delay spread and Doppler shift in the first tens of milliseconds - has been subject to a lot of studies, with the improvement of HF data modems in mind [Sanz-González, 2009]. Less literature is available on amplitude fading on a longer timescale [Furman, 2009]. The values mentioned come from practical experience, but correspond well with [Furman, 2009; Ads, 2013]. Multipath fading may produce a null at one of the antennas, while the other still has maximum signal. As a result of the spatial separation and different radiation patterns of the antennas, the signal variations are not necessarily correlated on each of the antennas that are compared. As a result of this, making only a few short-term signal strength comparisons would result in errors of up to $20 \mathrm{~dB}$. A better solution is proposed below.

\subsubsection{Proposed new evaluation method}

The following method, designed especially for the comparison of HF antennas, in-situ and with real signals and propagation, produces accurate and reproducible results:

A stable beacon transmitter is installed at sufficient distance from the antenna test site to generate strong NVIS signals and a negligible ground wave component. At the antenna test site, several antennas-under-test (AUTs) are installed in such a way that coupling between them is minimized. The constitution of the ground under each of the antennas that we compare is (roughly) the same. The AUTs are connected to a measurement receiver through an antenna switch. Both the measurement receiver and the antenna switch are computer controlled. A block diagram can be found in Figure 4.18. Signal strength and ambient noise level of each AUT are measured sequentially over a long period of stable NVIS propagation. The noise level is measured in the 'off' period of the transmitter and on adjacent channels. SNR is calculated for each measurement sample. One switch port is terminated in the characteristic impedance of the receiver, so that the receiver noise is also measured as a separate value. The distribution of the values measured on each AUT is plotted in one combined graph. When a large number of measurements is taken, each AUT shows a singlepeaked distribution, facilitating comparison of relative signal strength and SNR. This method remains very close to the practical use situation and produces accurate results. 


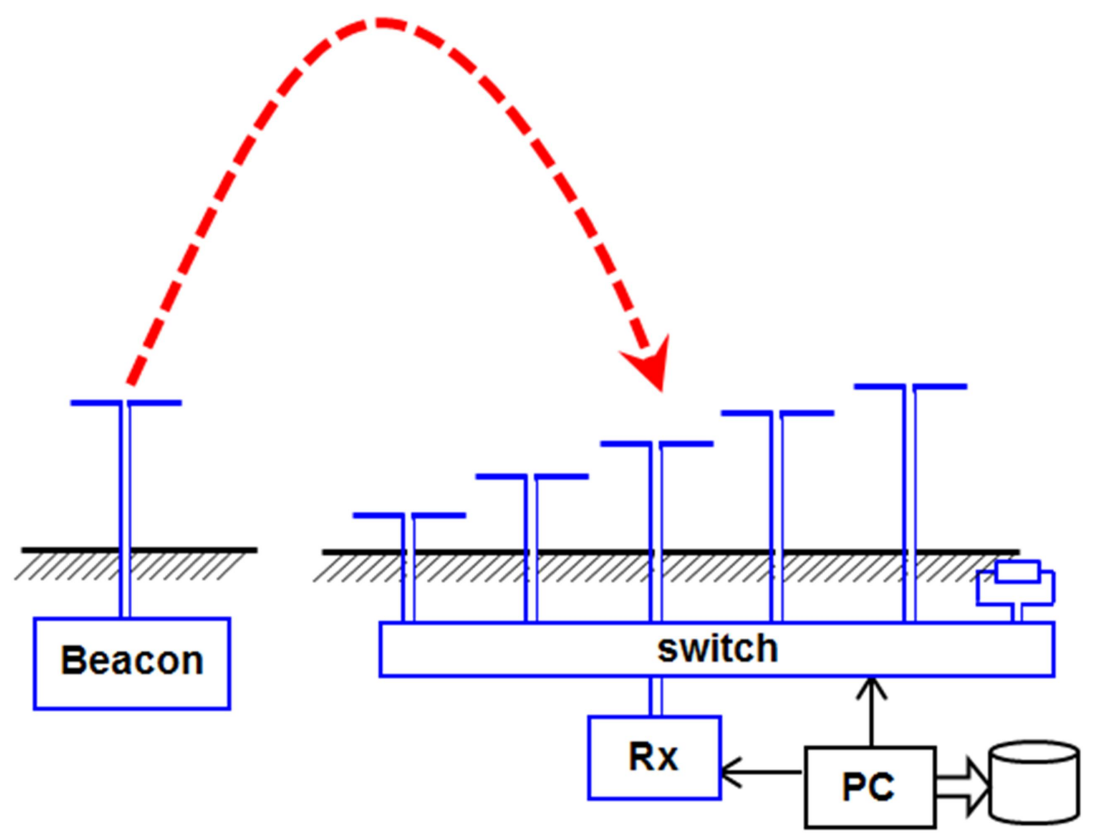

Figure 4.18 Block diagram of the proposed set-up for comparison of signal strength and SNR on 5 antennas mounted at different heights, in-situ and with real-life signals and propagation. The 6th port is terminated with a $50 \Omega$ load.

Hagn [1973] described a comparable method, using an ionosonde to send pulses upwards toward the zenith. The reflected pulses were received on the AUT and a reference antenna. The receiver was rapidly switched between these two antennas and the receiver output recorded with an ink paper recorder. A step attenuator was inserted in the feed line of the more efficient antenna and manually adjusted until the signal was equal on both antennas. The attenuator value then represented the antenna gain difference. His method provides relative antenna gain for signals arriving at zenith angles only and represents one single instant. It does not take into account the ionospheric variation over time and does not consider receive SNR.

The new method presented here profits from the advances in accuracy of the measurement receivers and the possibility to digitally store large numbers of measurements for post processing. The use of a carrier signal instead of short pulses simplifies the equipment needed. As the intervals over which data is collected are significantly longer than those used in [Hagn, 1973], the method presented here can be used to evaluate antenna performance under varying propagation conditions. Also both received signal strength and SNR can be evaluated using this method. Although designed for the verification of the optimum NVIS antenna heights in this investigation, it can easily be adapted for the evaluation of other antennas. When antennas intended for longer propagation paths are compared, the variance will be higher, just as will be the case in the actual application. The method will be excellent for the comparison of the in-situ performance of two or more antennas. 


\subsubsection{Practical realization of the proposed method}

The implementation of our experiment is described below and includes several practical measures that enhance accuracy. Our experiment took place from 1 April 2009 at 15:27h UTC to 2 April 2009 at 12:58h UTC, during the sunspot minimum between sunspot cycle 23 and 24, as can be seen in Figure 4.4. Consequently, the critical frequency fxF2 was very low, below $6.5 \mathrm{MHz}$. Therefore, to ensure that NVIS propagation was present during a significant part of the measurement period, the experiment was performed on $5.39 \mathrm{MHz}$. A special permission was obtained for the use of this frequency.

The beacon transmitter, constructed by A. J. Westenberg and capable of producing a continuous RF carrier output of 850 Watts during 24 hours, is shown in Figure 4.19. Transmitter output power stability was better than $0.1 \mathrm{~dB}$ over the entire measurement period. The frequency drift was less than $5 \mathrm{~Hz}(1 \mathrm{ppm})$. This transmitter was set-up near Lucaswolde, The Netherlands (approx. $54.2^{\circ} \mathrm{N}, 6.3^{\circ} \mathrm{E}$ ), feeding a horizontal half-wave dipole at 8.5 meters $(0.15 \lambda)$ above clay soil. The simulated antenna gain is $6.3 \mathrm{dBi}$. This results in $4.6 \mathrm{~kW}$ or $35.6 \mathrm{dBW}$ equivalent isotropically radiated power.

Approximately $127 \mathrm{~km}$ further to the South, near Eibergen, The Netherlands (approx. $52.1^{\circ} \mathrm{N}$, $6.6^{\circ} \mathrm{E}$ ), an open area with farmland soil was available for the installation of five horizontal half-wave dipole antennas. The antenna heights were selected from the previous simulations so that signal differences between the antennas were expected to be discernible. Chosen heights were $0.02 \lambda, 0.05 \lambda, 0.09 \lambda, 0.16 \lambda$, and $0.22 \lambda$. For $5.39 \mathrm{MHz}$, this corresponds with 3, 5, 9 and 12.5 meters. Each antenna was adjusted for resonance after installation to compensate for detuning due to ground proximity. Mutual coupling between the antennas was reduced by installing them end-to-end and in a straight line, as can be seen in Figure 4.20. Additionally, each antenna was connected to the antenna switch with a feed line of which the length was cut to an odd multiple of an electrical quarter wavelength. When not selected, the feed line was shorted by the switch. This short circuit transforms to a high impedance at the center of the dipole, effectively splitting it into two non-resonant halves.

Figure 4.21 shows the calibrated professional measurement receiver Rohde \& Schwarz FSMR26 used for our measurements. For absolute values, the combined measurement uncertainty is $0.3 \mathrm{~dB}$ for $95 \%$ confidence [R\&S, 2009]. Measurements on HF put high demands on receiver linearity due to the presence of strong broadcast signals. To verify that the measurement receiver was operated within its Intermodulation Free Dynamic Range (IFDR), the total power present at the antenna input was measured over 24 hours using a 20 $\mathrm{MHz}$ wide IF filter. Based on the results of this measurement, a band-pass filter was built and inserted at the receiver input to prevent overloading by strong out-of-band signals. 


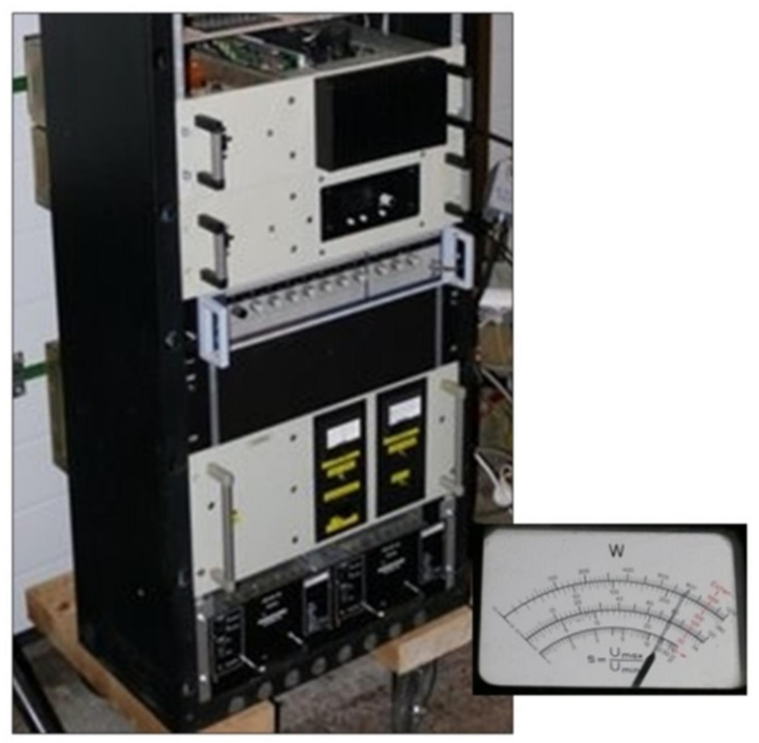

Figure 4.19 The beacon transmitter, capable of 850 Watt RF output continuous transmission on $5.39 \mathrm{MHz}$.

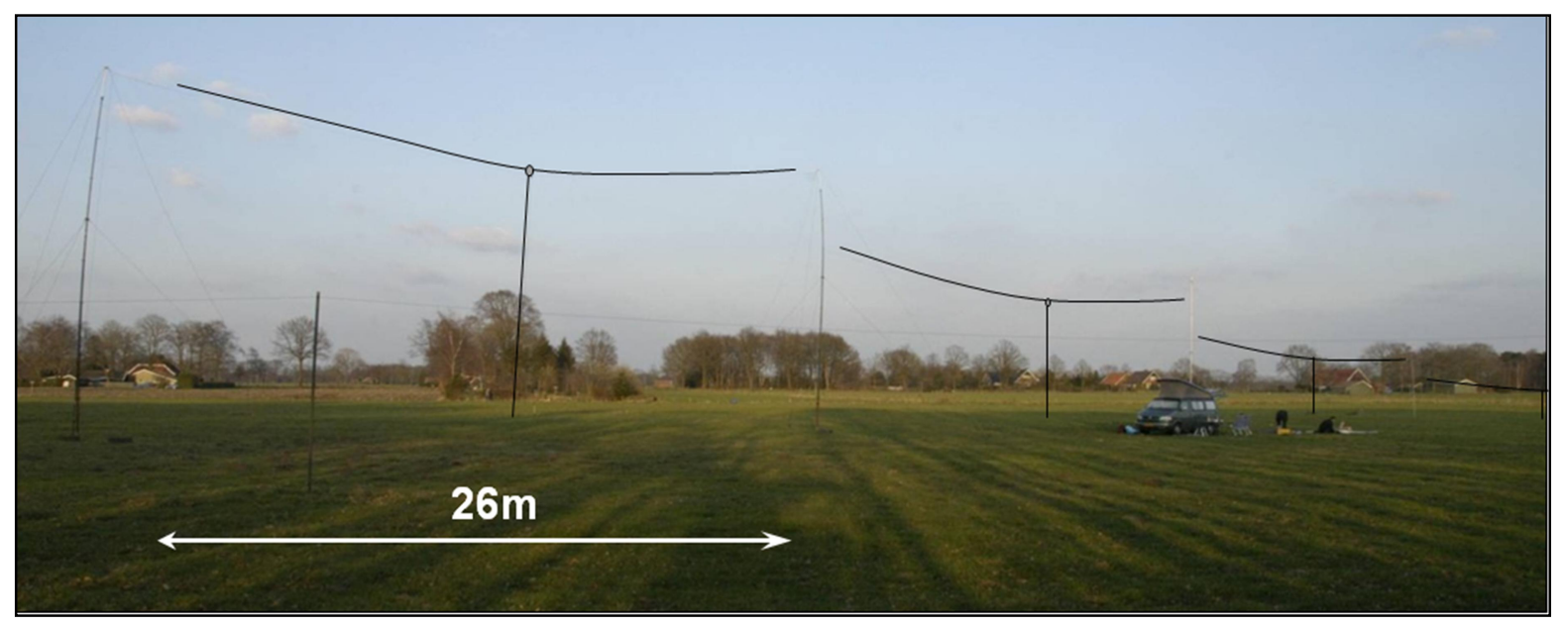

Figure 4.20 Three of the five Antennas-Under-Test.

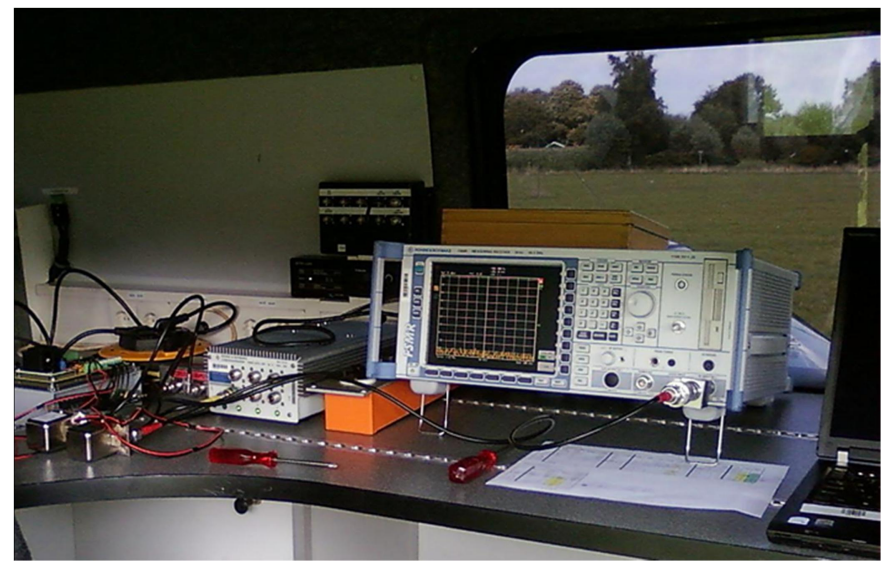

Figure 4.21 The Rohde \& Schwarz FSMR26 measurement receiver with coaxial switches and LabView automation. 
The transmitter and receiver drift was less than $5 \mathrm{~Hz}$ over a 24 hour interval. This - and the use of carrier (continuous wave) transmissions instead of the pulsed transmissions used by [Hagn, 1973] - made measurements using a $30 \mathrm{~Hz}$ IF filter possible, which provided high immunity to in-band interference. The measurements were automated using a custom measurement program written in LabView. The frequency was held free for the experiments. Even so, a series of $30 \mathrm{~Hz}$ frequency bins was measured around the receive frequency, so that a spectrogram was obtained which was used to monitor for any unexpected interfering signal that could compromise the measurements. The transmitter carrier was switched on and off in a precisely timed slow (1 minute) on/off cycle, synchronized to the DCF77 time signal transmitter. This made identification easy, and enabled observation of interference and noise measurement on the transmit frequency. As the spectrogram in Figure 4.22 shows, no interference was present on or near the measurement frequency.

\subsubsection{Empirical verification of the optimum NVIS transmit antenna height}

Using the set-up described in paragraph 4.5.2, measurements were started at 15:27h UTC and continued through $12: 58 \mathrm{~h}$ the next day. Within this time span, stable F-layer NVIS propagation was present during two intervals: from 15:27h to 19:05h and from 09:40h to 12:58h UTC. Figure 4.23 shows the received signal strength over time, and both intervals are marked as NVIS interval 1 and 2.

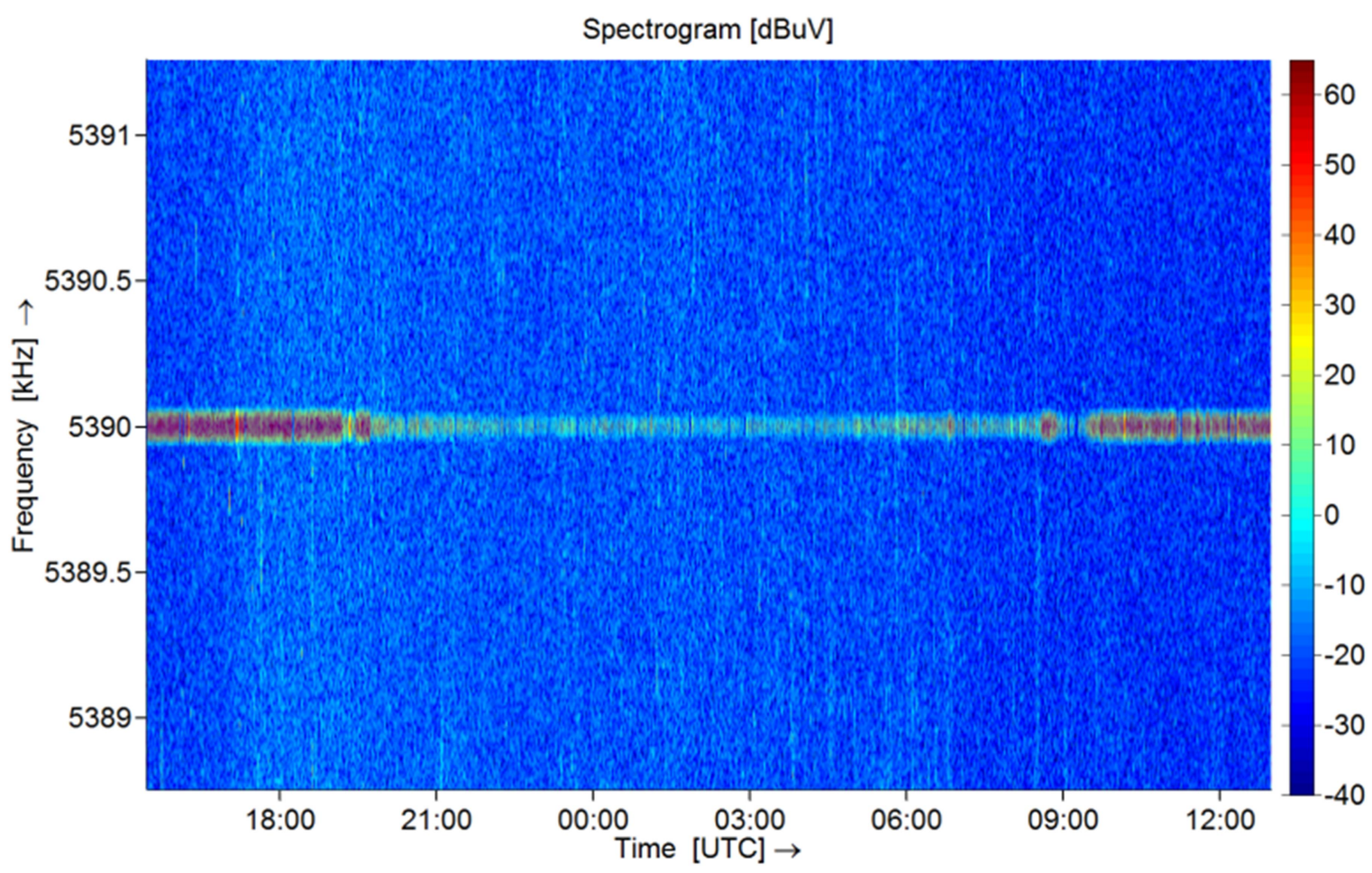

Figure 4.22 Spectrogram of the beacon and adjacent frequencies on one of the Antennas-Under-Test. 
The blue dashed vertical lines mark the interval in which the NVIS propagation switches from 'on' to 'off', in this particular case with some 'hesitation'. This 'hesitation' is caused by the short-term variation of $\mathrm{fxF} 2$ around the value needed to support NVIS propagation. In between NVIS interval 1 and 2 we had expected to observe no signal at all, or just a weak ground wave signal with little variance. Instead the signal remained clearly readable and measurable, far above the ambient noise, and it had all the properties of a skywave signal (fast fading and flutter).

Figure 4.24 shows the measured foF2 (red) and fxF2 (green). Solid lines represent data from the Dourbes ionosonde, dashed lines from the Juliusruh ionosonde. As can be seen, NVIS propagation is possible when fxF2 exceeds the operating frequency. This conforms to theory [Budden, 1985; Walden, 2009].

The measurements of NVIS intervals 1 and 2 are processed separately, as the measured average signal level in the morning is about $12 \mathrm{~dB}$ lower than in the evening. The distributions of the signal strength on each antenna are shown in Figures 4.25 (first NVIS interval) and Figure 4.26 (second NVIS interval). For each interval the total number of samples per antenna is 700 , histogram resolution is $0.1 \mathrm{~dB}$.

The measurement uncertainty of the receiver (R\&S FSMR26) for this frequency range is 0.3 $\mathrm{dB}(2 \sigma)$ for absolute values. As we compare antennas, the systematic error falls out of the equation and the remaining measurement uncertainty is better than $0.2 \mathrm{~dB}(2 \sigma)$. The measurement resolution is much smaller still $(0.01 \mathrm{~dB})$, and $66 \%$ of the measurements fall in a $0.1 \mathrm{~dB}$ window around the true value. Slight smoothing is applied using a sliding Gaussian window $(\sigma=0.15 \mathrm{~dB}, \mathrm{~N}=41)$. The smoothing parameters chosen are a compromise between resolution and smoothness of the curve.

The mean values of the measurements are marked in the graphs and their values are summarized in Table 4.3. The expected values that were derived from the simulations in paragraph 4.4.2 and shown in Table 4.1 are added for comparison. The signal strength measurements closely match the NVIS Antenna Gain values found by simulation. 


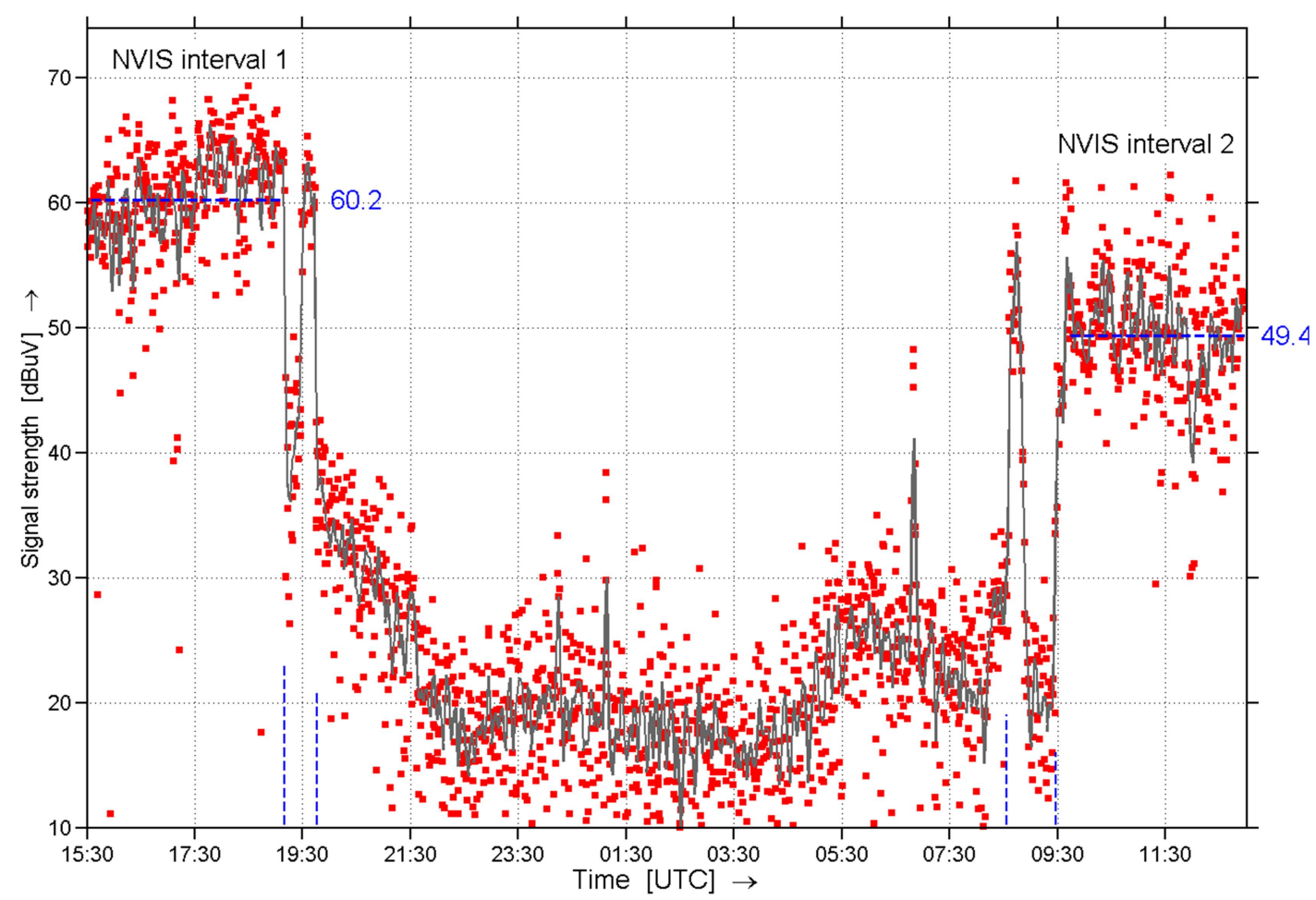

Figure 4.23 Signal strength vs. time on one of the Antennas-Under-Test. Both NVIS intervals are indicated. A grey line shows the 8 minute floating average. The blue vertical dashed lines show the interval in which the NVIS propagation switches between "on" and "off".

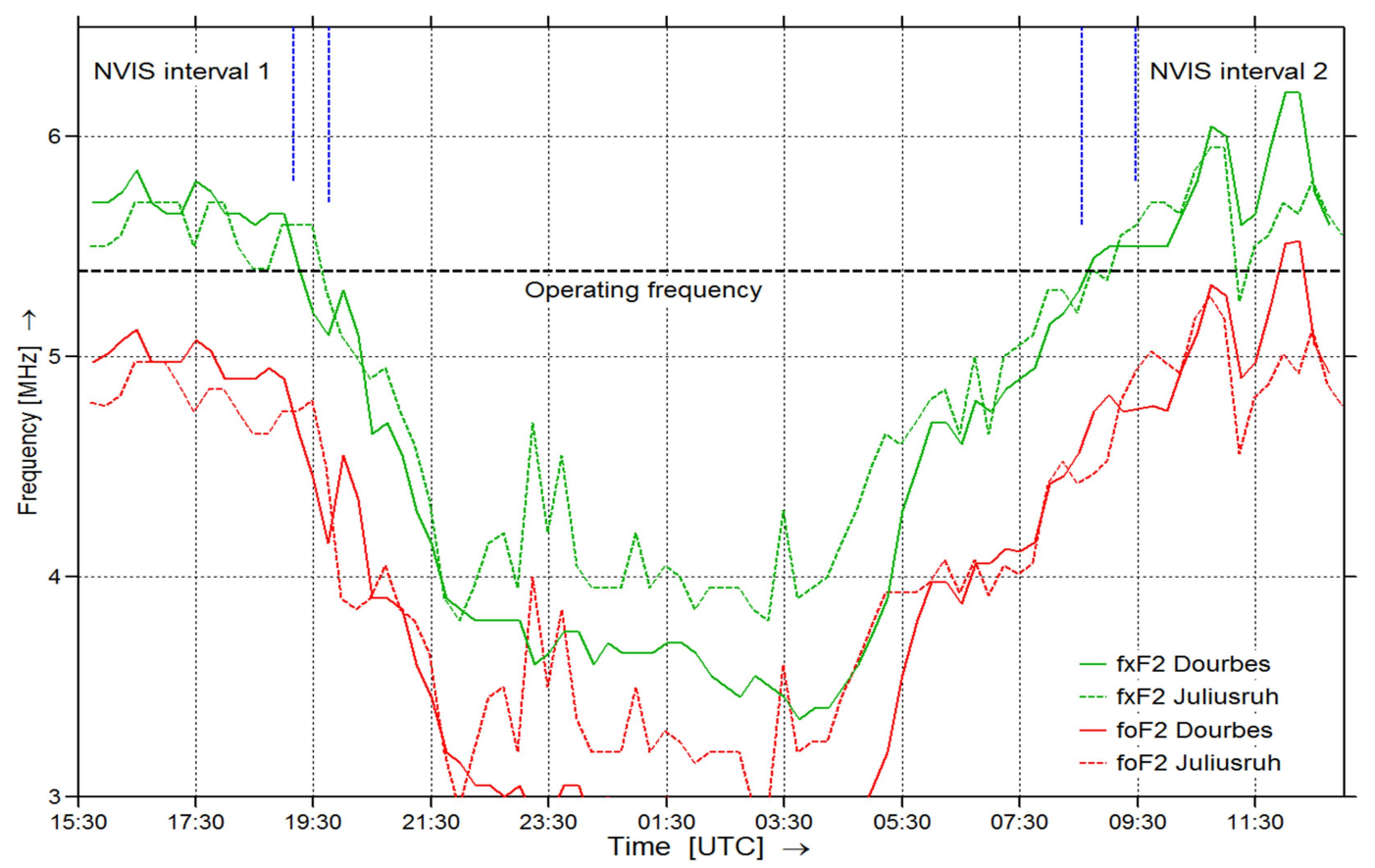

Figure 4.24 Measured foF2 (red) and fxF2 (green) over time. Solid lines represent data from the Dourbes ionosonde, dashed lines from the Juliusruh ionosonde. The blue vertical dashed lines show the interval in which the NVIS propagation switches between "on" and "off". 


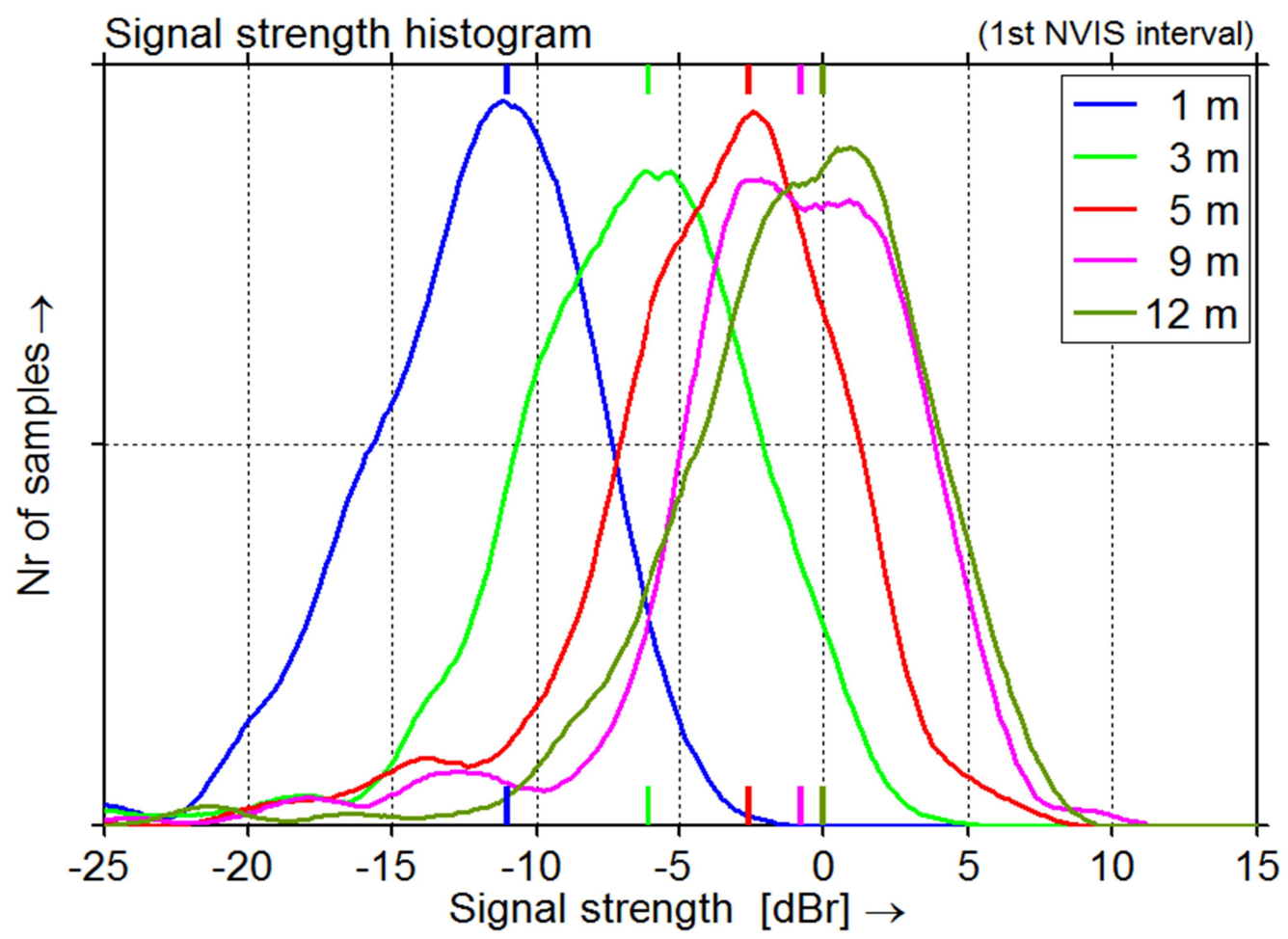

Figure 4.25 Histogram of the signal strength on 5 identical antennas mounted at different heights, first NVIS interval. $0 \mathrm{dBr} \equiv 61.5 \mathrm{~dB} \mu \mathrm{V}$.

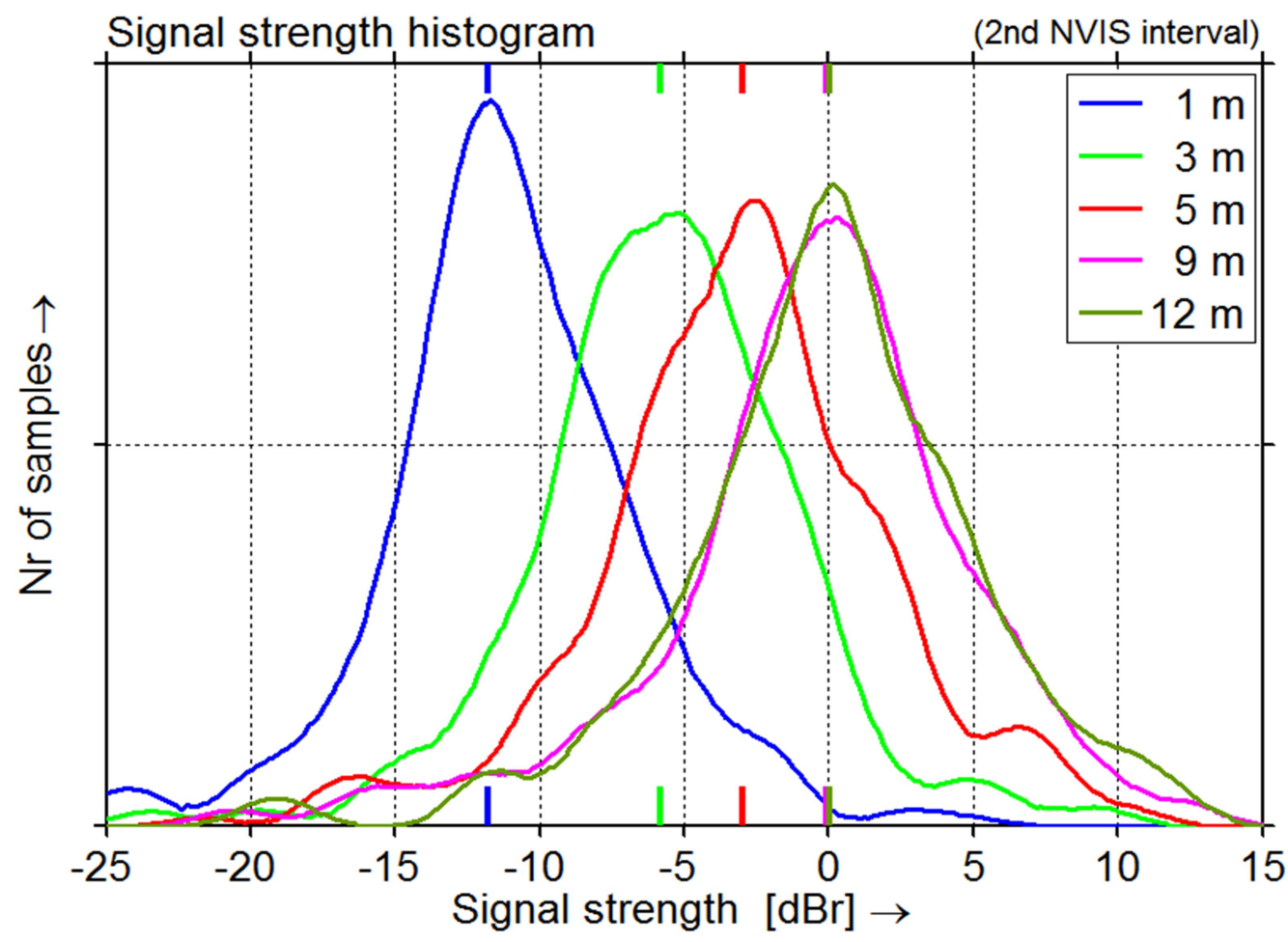

Figure 4.26 Histogram of the signal strength on 5 identical antennas mounted at different heights, second NVIS interval. $0 \mathrm{dBr} \equiv 49.5 \mathrm{~dB} \mu \mathrm{V}$. 
Table 4.3 Comparison of expected and measured NVIS Antenna Gain for a dipole antenna above farmland soil.

\begin{tabular}{|cc|c|c|c|}
\hline \hline \multirow{2}{*}{ Antenna height } & \multicolumn{3}{|c|}{ NVIS Antenna Gain } \\
\cline { 3 - 6 } & & Expected & Meas.1 & Meas.2 \\
\hline $12.5 \mathrm{~m}$ & $0.22 \lambda$ & $-0.2 \mathrm{dBr}$ & $0.0 \mathrm{dBr}$ & $0.0 \mathrm{dBr}$ \\
$9 \mathrm{~m}$ & $0.16 \lambda$ & $-0.0 \mathrm{dBr}$ & $-0.8 \mathrm{dBr}$ & $0.0 \mathrm{dBr}$ \\
$5 \mathrm{~m}$ & $0.09 \lambda$ & $-1.5 \mathrm{dBr}$ & $-2.6 \mathrm{dBr}$ & $-4.0 \mathrm{dBr}$ \\
$3 \mathrm{~m}$ & $0.05 \lambda$ & $-5.0 \mathrm{dBr}$ & $-6.1 \mathrm{dBr}$ & $-5.8 \mathrm{dBr}$ \\
$1 \mathrm{~m}$ & $0.02 \lambda$ & $-12.0 \mathrm{dBr}$ & $-11.0 \mathrm{dBr}$ & $-11.8 \mathrm{dBr}$ \\
\hline \hline
\end{tabular}

\subsubsection{Empirical verification of the optimum NVIS receive antenna height}

On each of the antennas we measured both signal strength and ambient noise level to calculate SNR. This is done as follows: During the measurements the received noise was measured in the 1 minute intervals that the transmitter was off, but also continuously in the adjacent frequency bins ( $30 \mathrm{~Hz}$ channels). The ambient noise can only be measured correctly if the receiver noise floor is low enough. The noise contribution of the receiver itself was measured on the 6th port of the antenna switch which was terminated with a $50 \Omega$ load, as was shown in Figure 4.18. Throughout the experiment the measured noise power was 7-20 $\mathrm{dB}$ higher than the receiver noise floor on the highest antenna, and 3-11 dB on the lowest antenna. The latter, representing the worst case, is shown in Figure 4.27. The true value of the ambient noise was calculated from the measured ambient noise and the measured receiver noise on a sample-by-sample basis and used in the subsequent analysis.

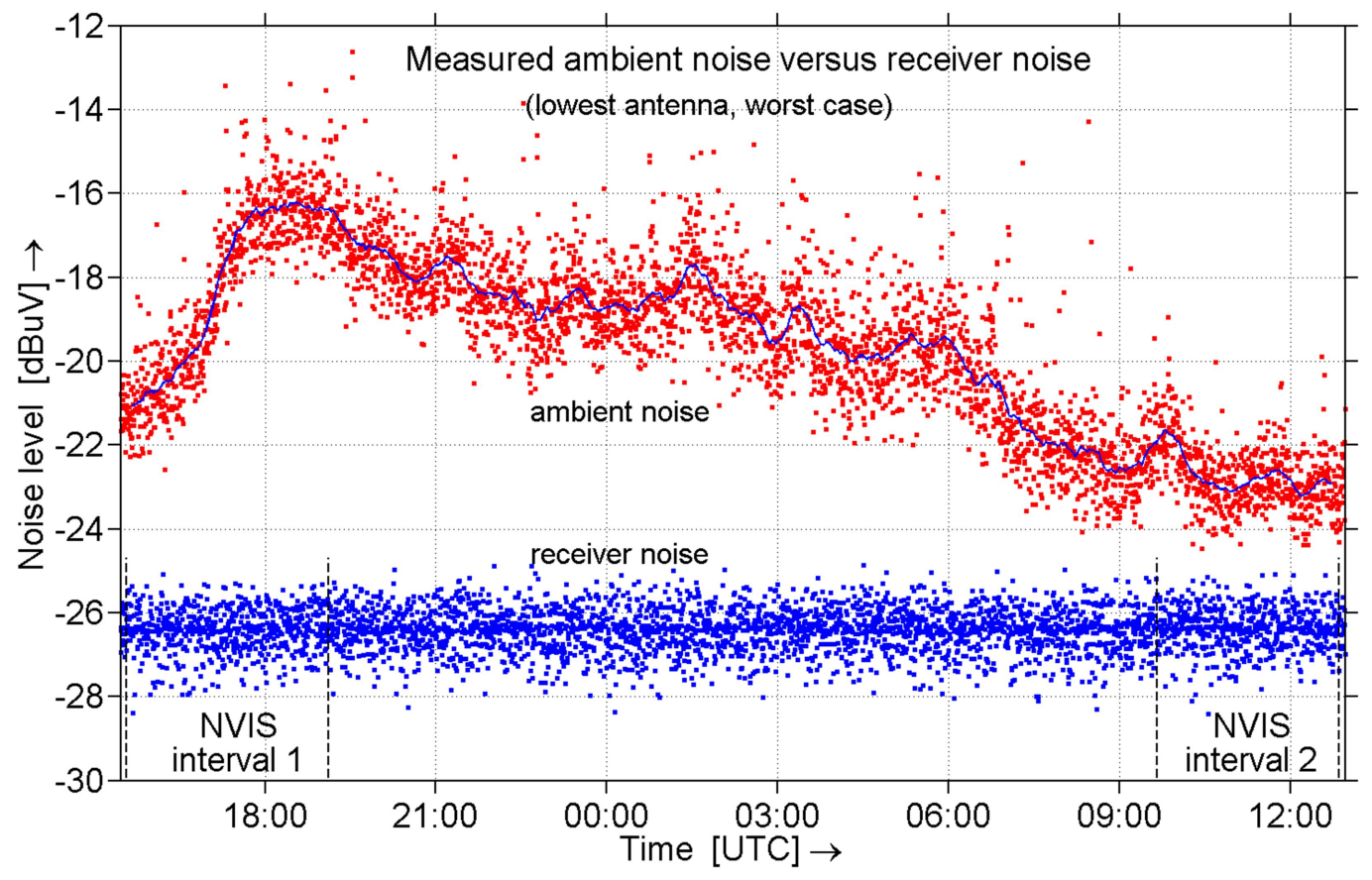

Figure 4.27 Measured ambient noise on the lowest Antennas-Under-Test (worst case) vs. time, shown as red pixels, with superimposed 12 minute average. The blue pixels show the receiver noise. 
The measured ambient noise samples and the measured signal strength samples were used to calculate the signal-to-noise ratio (SNR) per sample. These values were then processed it in the same way as has been done for the received signal strength samples in the previous paragraph. Again the measurements for both NVIS intervals, from 15:27h to 19:05h and from 09:40h to 12:58h UTC, are processed separately. The distributions of the SNR on each antenna are shown in Figures 4.28 and 4.29. For each interval the total number of samples per antenna is 700 , histogram resolution is $0.1 \mathrm{~dB}$. Slight smoothing is applied using a sliding Gaussian window $(\sigma=1.3 \mathrm{~dB}, \mathrm{~N}=41)$. Again the smoothing parameters chosen are a compromise between resolution and smoothness of the curve.

The mean values of the measurements are marked in the graphs and their values are summarized in Table 4.4. The NVIS Directivity values derived from the simulations in paragraph 4.4.3 and shown in Table 4.2 are added for comparison. When a uniform spatial distribution of the ambient noise is assumed, a direct relationship between the two is expected. However, as Table 4.4 shows, the optimum NVIS receive antenna height found empirically is slightly higher than simulation suggested: around $0.16 \lambda$ instead of $0.09 \lambda$. Also the measured SNR values decrease faster with decreasing antenna height than was expected from the simulations.

The theoretical optima were obtained assuming uniform spatial distribution of the ambient noise, as no a priori knowledge is available about the azimuthal direction and elevation angle from which natural noise and man-made noise would arrive at an ad-hoc receive site. When, however, a large number of man-made noise sources arrive via skywave, e.g. from a city or an industrial area, specific spatial directions will contribute more noise than others. Also, if a few dominant man-made noise sources are present at close range, their signals will arrive via ground wave and from specific angles. This has not been considered in the simulations and could possibly explain the difference between the measured and simulated optima.

Table 4.4 Comparison of expected and measured NVIS SNR for a dipole antenna above farmland soil.

\begin{tabular}{|cc|c|c|c|}
\hline \hline \multirow{2}{*}{ Antenna height } & \multicolumn{3}{|c|}{ NVIS SNR } \\
\cline { 3 - 5 } & & Expected & Meas.1 & Meas.2 \\
\hline $12.5 \mathrm{~m}$ & $0.22 \lambda$ & $-0.8 \mathrm{dBr}$ & $-1.6 \mathrm{dBr}$ & $-0.3 \mathrm{dBr}$ \\
$9 \mathrm{~m}$ & $0.16 \lambda$ & $-0.2 \mathrm{dBr}$ & $0.0 \mathrm{dBr}$ & $0.0 \mathrm{dBr}$ \\
$5 \mathrm{~m}$ & $0.09 \lambda$ & $0.0 \mathrm{dBr}$ & $-0.6 \mathrm{dBr}$ & $-0.9 \mathrm{dBr}$ \\
$3 \mathrm{~m}$ & $0.05 \lambda$ & $-0.3 \mathrm{dBr}$ & $-1.8 \mathrm{dBr}$ & $-4.3 \mathrm{dBr}$ \\
$1 \mathrm{~m}$ & $0.02 \lambda$ & $-0.8 \mathrm{dBr}$ & $-2.1 \mathrm{dBr}$ & $-6.5 \mathrm{dBr}$ \\
\hline \hline
\end{tabular}




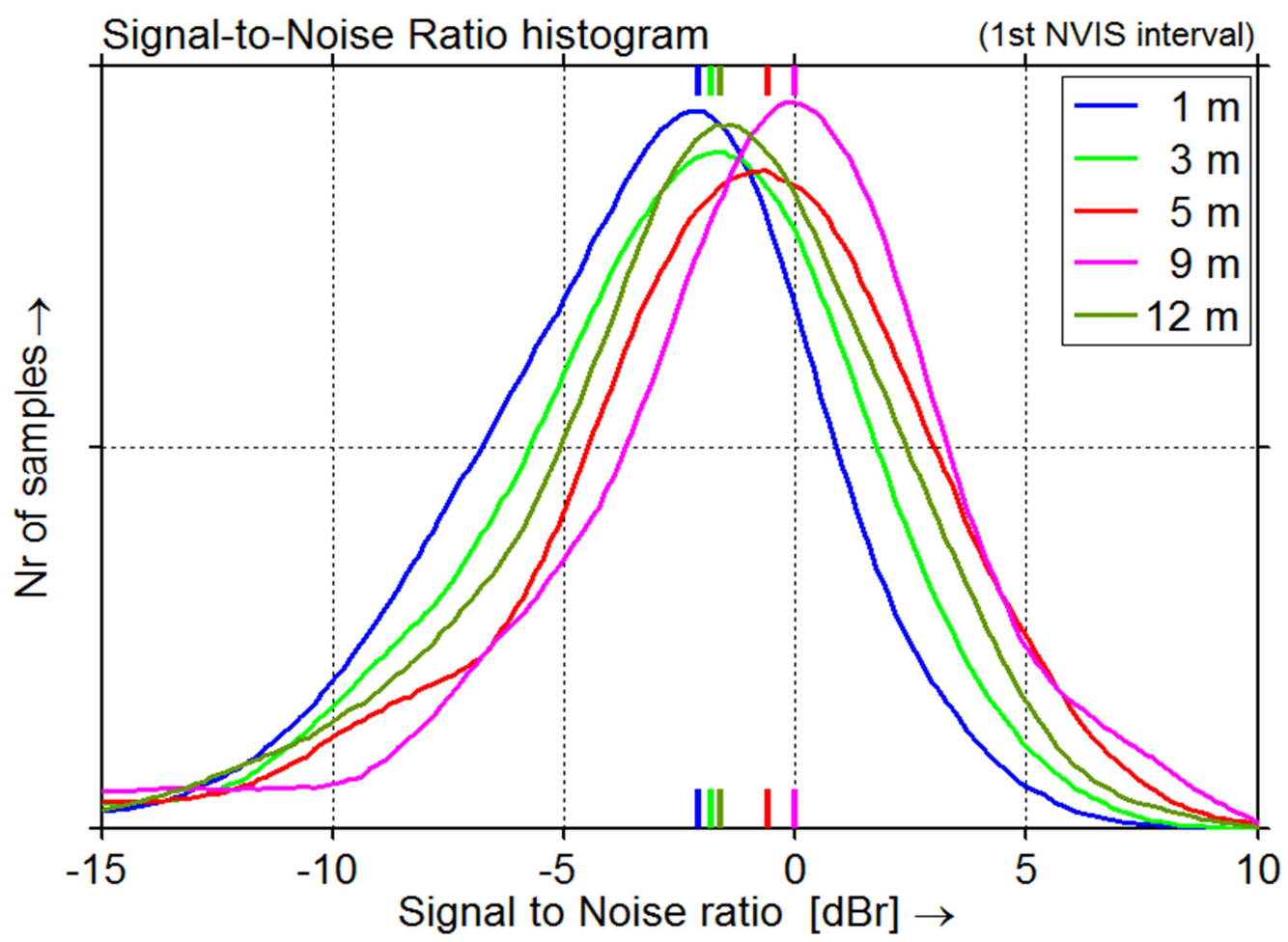

Figure 4.28 Histogram of the signal-to-noise ratio (SNR) on 5 identical antennas mounted at different heights, first NVIS interval. $0 \mathrm{dBr} \equiv 71.9 \mathrm{~dB}$ SNR.

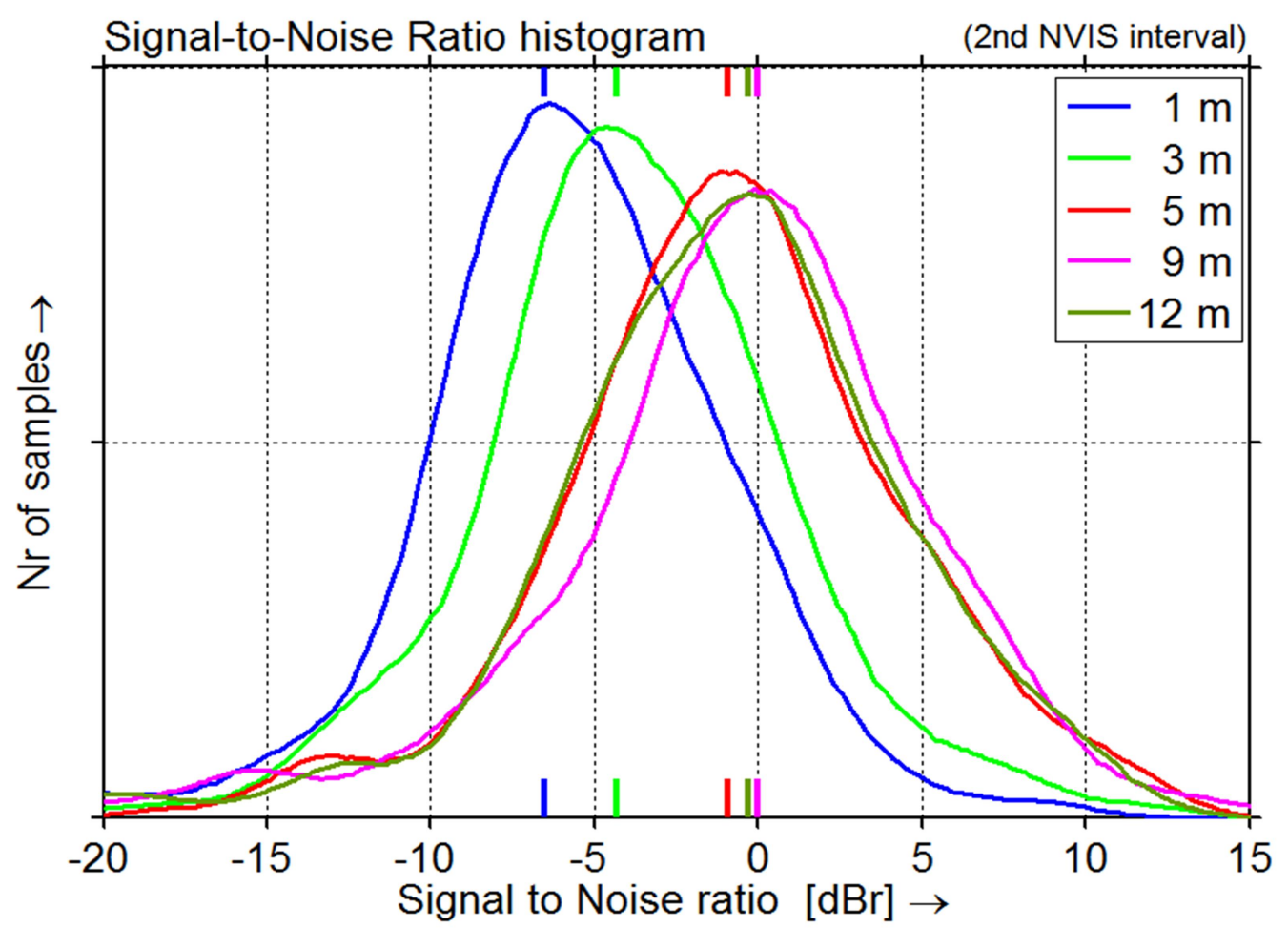

Figure 4.29 Histogram of the signal-to-noise ratio (SNR) on 5 identical antennas mounted at different heights, second NVIS interval. $0 \mathrm{dBr} \equiv 69.3 \mathrm{~dB}$ SNR. 


\subsection{ANALYSIS AND DISCUSSION}

The research described in this chapter was performed on $5.39 \mathrm{MHz}$, on one location and one instant within the solar cycle. A coverage area size of $150 \mathrm{~km}$ was presumed. This section discusses the applicability for other scenarios.

\subsubsection{Sensitivity to frequency and coverage area size}

All simulations in Section 4.4 were done on 5.39 $\mathrm{MHz}$. To assess the influence of the operating frequency, additional simulations were done on $3 \mathrm{MHz}$ and $15 \mathrm{MHz}$ for all soil types specified in Table 4.1 and Table 4.2. The effect on directivity over this frequency range is smaller than $0.5 \mathrm{~dB}$ for all heights and soil types. The absolute antenna gain decreases by 0.1 to $2 \mathrm{~dB}$ for a frequency change from 3 to $15 \mathrm{MHz}$, but the optimum heights are not significantly changed.

The optima found in Section 4.4 are for NVIS elevation angles between $70^{\circ}$ and $90^{\circ}$, targeting a coverage area with a radius of $150 \mathrm{~km}$. If a larger area is targeted, this optimum height will be slightly higher. Increasing height will result in lowering of the elevation angle at the cost of reduction in antenna gain for higher elevation angles. As $D_{\text {NVIS }}$ and $G_{N V I S}$ are the average gain and directivity over a range of elevation angles, the effect is not very sensitive to small changes in coverage area. For much larger areas however, e.g. to a radius of $500 \mathrm{~km}$ the influence will be more notable and the procedure described in paragraphs 4.4.2 and Section 4.3 can be followed find the applicable optima.

\subsubsection{Influence of solar activity}

As the measurements were carried during one day during a sunspot minimum, this raises the question on the applicability of the results to other parts of the solar cycle. First of all, the elevation angles will vary with the sunspot number, as was shown in Figures 4.5 and 4.6. However, the variation is not important for NVIS elevation angles as long as a frequency is chosen favoring F-layer propagation.

The verification measurements are performed during a solar cycle minimum, and low sunspot activity obliges all shortwave users to use lower frequencies. This generally results in congestion in the lower part of the shortwave during sunspot minima, which would result in the 'worst case' situation considering interference. However, this aspect has no influence on the measured SNR values, as they are all measured in a clear channel. Optimized NVIS Directivity will of course also provide the lowest susceptibility to interference of non-NVIS radio signals, i.e. interference signals arriving via lower elevation angles, but this has not been measured. It is true that ambient noise distributions may be different around the sunspot maximum than at the sunspot minimum, but this has not been researched. 


\subsubsection{Comparison with other research}

Austin [2009] used a simple formula using geometric optics to relate antenna height to the elevation angle at which maximum radiation occurs. He starts assuming perfect conducting ground underneath the antenna and arrives at an optimum height of $\lambda / 4$ for perfect conducting ground, as was expected. He then modifies his formula with an empirical correction factor, derived from NEC computations over rural ground ( $\sigma \approx 5 \mathrm{mS} / \mathrm{m}, \varepsilon r \approx 13$ ). He then arrives at an optimum height of $0.22 \lambda$ for NVIS angles, which corresponds very well with the values found here. Austin states that "this particular result is not overly sensitive to ground conductivity changes by an order of magnitude. It is somewhat more sensitive to a change in relative permittivity and also more so at lower frequencies," but does not substantiate this statement with calculations or experiments.

Extensive military research was performed by [Barker, 1971] in several terrain and vegetation types. They state in the abstract that "...that the effect of the antenna height is the most significant variable" influencing the radiation patterns. Their research focused on the antenna gain at zenith angle, not the average antenna gain over all NVIS angles. However, in Figures 37 and 41 of their report they show antenna gain values that are within $1 \mathrm{~dB}$ of the values we simulated, and the decrease in antenna gain when lowering the antenna from $0.20 \lambda$ to $0.02 \lambda$ is resp. $13.5 \mathrm{~dB}$ (open terrain) and $10 \mathrm{~dB}$ (tropical forest). These results align with our findings.

\subsection{CONCLUSIONS}

The relationship between NVIS elevation angles and skip distance is simulated using ionospheric ray tracing software and verified by measurement using a professional Radio Direction Finder. Both measurements and simulations confirm the high elevation angles involved in NVIS, ranging from $70^{\circ}$ to $90^{\circ}$ for a coverage area with $150 \mathrm{~km}$ radius. The measurements show that the dominance of NVIS over ground wave propagation starts at a short distance from the transmitter: e.g. $20 \mathrm{~km}$ at a frequency of $7 \mathrm{MHz}$. For these NVIS elevation angles, the optimum height above ground of horizontal half-wave dipole antennas is sought. To facilitate antenna optimization, NVIS Antenna Gain and NVIS Directiviy are defined first as the average gain and average directivity over these NVIS elevation angles $\left(70^{\circ}\right.$ to $\left.90^{\circ}\right)$. NVIS Antenna Gain must be optimized for transmission; NVIS Directivity must be optimized for best reception.

NEC 4.1 simulations show an optimum NVIS transmit antenna height ranging from $0.18 \lambda$ to $0.22 \lambda$ for most soil types. The NVIS antenna gain at $0.02 \lambda$ is $12 \mathrm{~dB}$ lower than the optimum. Above sea water the optimum height is $0.13 \lambda$. Simulation shows that the receive antenna height is not critical: NVIS Directivity varies only $0.8 \mathrm{~dB}$ over a range of $0.02 \lambda$ to $0.22 \lambda$.

To verify these results, an empirical evaluation method for NVIS antenna performance in the presence of HF fading measurement method is proposed and demonstrated. The optimum 
NVIS transmit antenna height is strongly supported by these measurements. The optimum NVIS receive antenna height found empirically is slightly higher than simulation suggested, around $0.16 \lambda$ for farmland soil, and is slightly more critical than expected: 2 to $6 \mathrm{~dB}$ deterioration of the SNR occurs when the antenna is lowered to $0.02 \lambda$.

We may conclude that, in situations where the last few $\mathrm{dB}$ really matter, it is worthwhile to consider the optimum antenna height found here. The difference may be up to $12 \mathrm{~dB}$, which is substantial, and the investment is small. An example could be the establishment of a fixed or ad-hoc base station for emergency communications, meant to communicate with small battery operated stations with suboptimal antennas in the field.

On the other hand one could conclude that even very low dipole antennas that yield not more than $-4.9 \mathrm{dBi}$ at a height of $0.02 \lambda$ will outperform the $-17 \mathrm{dBi}$ of a whip antenna on a car [Hagn, 1970]. If propagation is favorable, the $12 \mathrm{~dB}$ antenna loss can be offset with an increase of transmit power of the station at the other end of the radio link, provided that station also has the reception capability to match it. However, in any situation where radio communication is essential and peak performance is required, the optima found in this research are recommended. 


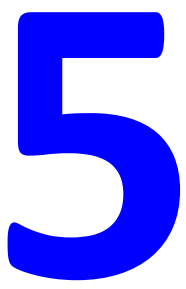

\section{A novel method for the evaluation of polarization and hemisphere coverage of $\mathrm{HF}$ radio noise measurement antennas}

Abstract-In HF (3-30 MHz) communications the ambient electromagnetic background noise or 'radio noise' generally is the limiting factor in reception. Radio noise measurements are needed for spectrum pollution control and to provide reference levels for radio system design. This chapter discusses the influence of the measurement antenna on the measurement results. A novel method to compare the polarization and hemisphere coverage of radio noise measurement antennas is proposed. This method is used to compare three HF radio noise measurement antennas. A novel omni-directional antenna is introduced which has improved sensitivity for high angle skywave signals and which is impartial to the polarization of the incoming wave.

Published as: B. A. Witvliet, E. van Maanen, M. J. Bentum, C. H. Slump, R. Schiphorst, "A Novel Method for the Evaluation of Polarization and Hemisphere Coverage of HF Radio Noise Measurement Antennas," presented at IEEE International Symposium on Electromagnetic Compatibility, Dresden, Germany, August 2015. 
B. A. Witvliet

Interaction of Antenna and Propagation Mechanism 


\section{5 - A novel method for the evaluation of polarization and hemisphere}

coverage of HF radio noise measurement antennas

\subsection{INTRODUCTION}

For professional radio communication receivers in the HF frequency range (3-30 MHz), the lower reception threshold is generally determined by the level of the ambient electromagnetic noise ('radio noise'), rather than by the noise floor of the receiver itself [Kraus, 1988, p. 766]. This radio noise consists of:

(a) natural noise caused by atmospheric discharges and radiation from extraterrestrial objects, and

(b) man-made noise caused by unwanted emissions of electronic equipment and electrical machinery.

In urban and industrial surroundings the man-made noise level exceeds that of the natural noise, sometimes with a 20-40 dB margin [Leferink, et al., 2010]. In quiet rural locations, natural noise may be observed. Both man-made noise and natural noise vary with frequency and time. Over the years, extensive radio noise research [Thomas, 1950; CCIR Report 65, 1957; Crichlow, 1960, 1966; Clarke, 1962; Spaulding, 1974; CCIR Report 322-2, 1983; CCIR Report 258-5, 1990] has provided us with empirically obtained reference levels for natural noise and man-made noise, which can be used for HF radio link planning [ITU-R Rec. P.372-11, 2013]. Considering the large variability of the radio noise with time and location, and the global coverage of this Recommendation, these reference levels must be considered indicative. If the reception threshold at a receive location is to be calculated, or a quiet receive location is to be selected, local radio noise measurements must be performed. Local and regional radio noise measurements must also be performed to provide feedback to EMC (Electromagnetic Compatibility) enforcement authorities, as rising [Leferink, 2010] or steadily high man-made noise levels could indicate failing EMC management.

Traditionally, radio noise measurements are performed using a short vertical whip antenna [Potter, 1932; Thomas, 1950; CCIR Report 65, 1957; Crichlow, 1960], primarily as this simple antenna provides wideband azimuthal coverage over a large frequency range. When the radiator is kept shorter than a quarter wavelength, and when ohmic losses in the antenna are compensated for in the measurement results, comparison between measurements is possible. It has become the standard antenna in the ITU radio noise reports, although with different descriptions [CCIR Report 65, 1957; ITU-R Rec. P.372-6, 1994; ITU-R Rec. P.372-11, 2013]. In this chapter we will indicate this antenna as Short Lossless Vertical Monopole (SLVM). While the SLVM provides good azimuthal coverage, it has limited sensitivity for radio waves arriving at high angles and it has a polarization mismatch with the incoming skywave noise. Consequently, the skywave component of the radio noise is underestimated in most radio noise measurements. For research into Near Vertical Incidence Skywave (NVIS) propagation [Burgess, 1999] and communication over the NVIS channel [Hervás, 2013], radio 
noise arriving at steep elevation angles (see Chapter 4) must be taken into account. Therefore a different measurement antenna is desired. The research question of this chapter is therefore: how to include the skywave noise components in radio noise measurements?

This chapter discusses the radio wave propagation from noise source to receiver and the influence of the radiation pattern and polarization response of the measurement antenna on the measurement results. It introduces a novel method that can be used to compare the performance of antennas used for radio noise measurement on both hemisphere coverage and spatial angle dependent polarization response. Using this method an improved synthetic antenna is discussed, which has a better response to high angle skywave signals and which responds equally well to all wave polarizations.

The chapter is structured as follows: Section 5.2 describes the different propagation paths from the HF radio noise source to the receive antenna, and the resulting elevation angle and polarization of the incoming wave. Section 5.3 introduces a novel method for the comparison of radio noise measurement antennas, and uses this method to compare a few HF antennas used for radio noise measurement. The observations result in an improved antenna design, which is described in Section 5.4. This chapter concludes with a discussion and topics for further research.

\subsection{HF RADIO NOISE PROPAGATION}

In urban and residential areas, HF man-made noise is much stronger than the underlying natural noise. Coupling of the noise source and the receive antenna occurs via ground wave or line-of-sight propagation, as is shown in Figure 5.1. The ground wave component has predominantly vertical polarization. The polarization of the line-of-sight component is mixed.

In quiet rural areas man-made noise is almost absent and the natural radio noise is observed: atmospheric noise, noise from the ionosphere and noise from extraterrestrial sources. Atmospheric noise ('atmospherics', 'static'), caused by lightning discharges, arrives both via line-of-sight and via ionospheric radio wave propagation [Horner, 1953] as shown in Figure 5.2. Distance from source to receiver may be as high as $3000 \mathrm{~km}$ [Watson Watt, 1938] and multiple sources accumulate. Elevation angles are low to intermediate and polarization is vertical to elliptical. The D-region of the ionosphere, being a hot plasma, emits faint thermal noise in the lower HF spectrum [Pawsey, 1957]. This emission is unpolarized and arrives at high angles.

Electromagnetic noise from extraterrestrial radio sources, such as the sun [Ryle, 1948], Jupiter [Kaiser, 1993] and the Milky Way [Jansky, 1938; Reber, 1944] arrives at the earth through the ionospheric iris [Rush, 1977], which aperture is very wide at $30 \mathrm{MHz}$, but decreases with frequency, closing completely when the frequency drops below the equivalent vertical frequency of the ionosphere [Davies, 1990, pp. 157-158]. Therefore, the 
elevation angle of extra-terrestrial noise is high between 3 and $10 \mathrm{MHz}$, and variable at higher frequencies. The noise emitted by the sun has circular polarization [Ryle, 1948]; most other extraterrestrial sources emit unpolarized noise.

The emissions of a large number of strong man-made noise sources, e.g. from a city, appear as one massive accumulated noise emission, of which the individual components cannot be distinguished. This accumulated man-made noise is reflected by the ionosphere, thereby contributing to a rise in the radio noise level, even in more remote areas. In this chapter we refer to this noise component as 'skywave radio noise'. This component may arrive from any spatial angle and will have variable elliptic polarization, as will be shown in Chapter 8.

Figure 5.3 shows the frequency-time diagram of a 24 hour radio noise measurement in a frequency segment between 7.8 and $8.3 \mathrm{MHz}$ in a rural area in The Netherlands. The frequency segment was selected for low activity of intended transmitters, although a few of such radio signals can be identified. All other signals are radio noise. Nearby lightning discharges can be recognized by their wideband spectrum.

Between 00:00h and 03:00h UTC a single local man-made noise source stands out of the background radio noise. The background radio noise level rises around 06:00h UTC and decays around 15:00h UTC. This corresponds with the ionospheric (skywave) propagation for this frequency range at the moment of observation. Between 22:00h and 24:00h UTC, a large number of man-made noise sources is active and only their cumulative noise emission can be seen. The individual noise sources can no longer be identified in the spectrum.

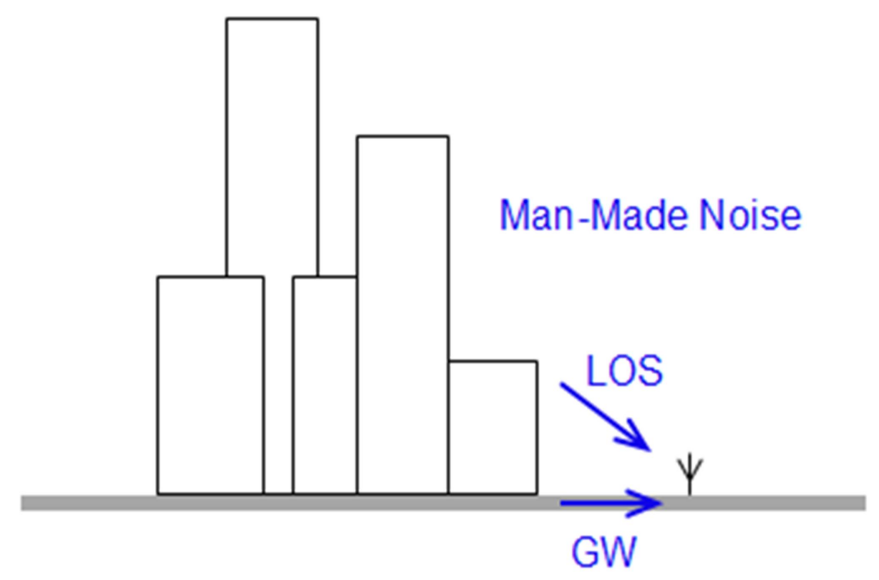

Figure 5.1 Man-made noise arriving at an urban receiver via line-of-sight (LOS) and ground wave (GW) propagation. 


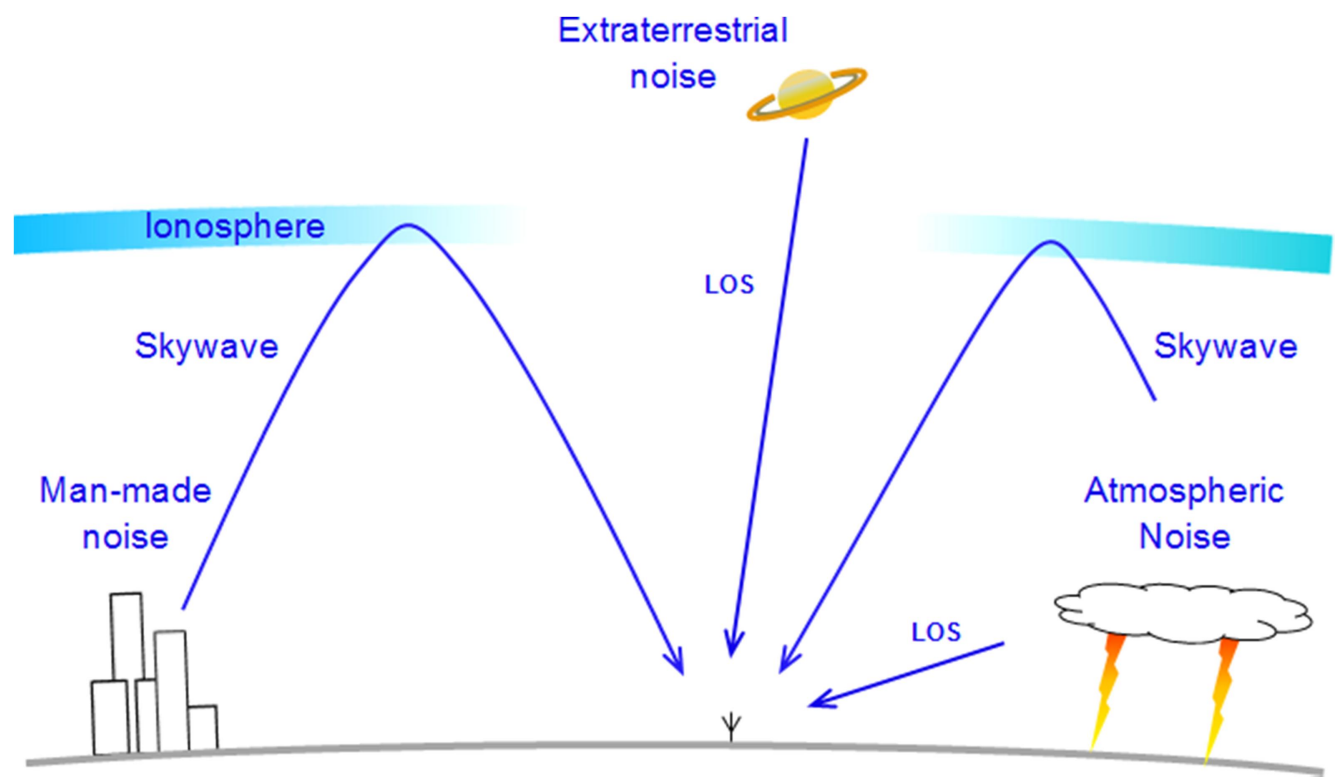

Figure 5.2 Several types of radio noise arriving at a receiver in a quiet rural area via skywave and line-of-sight (LOS) propagation.

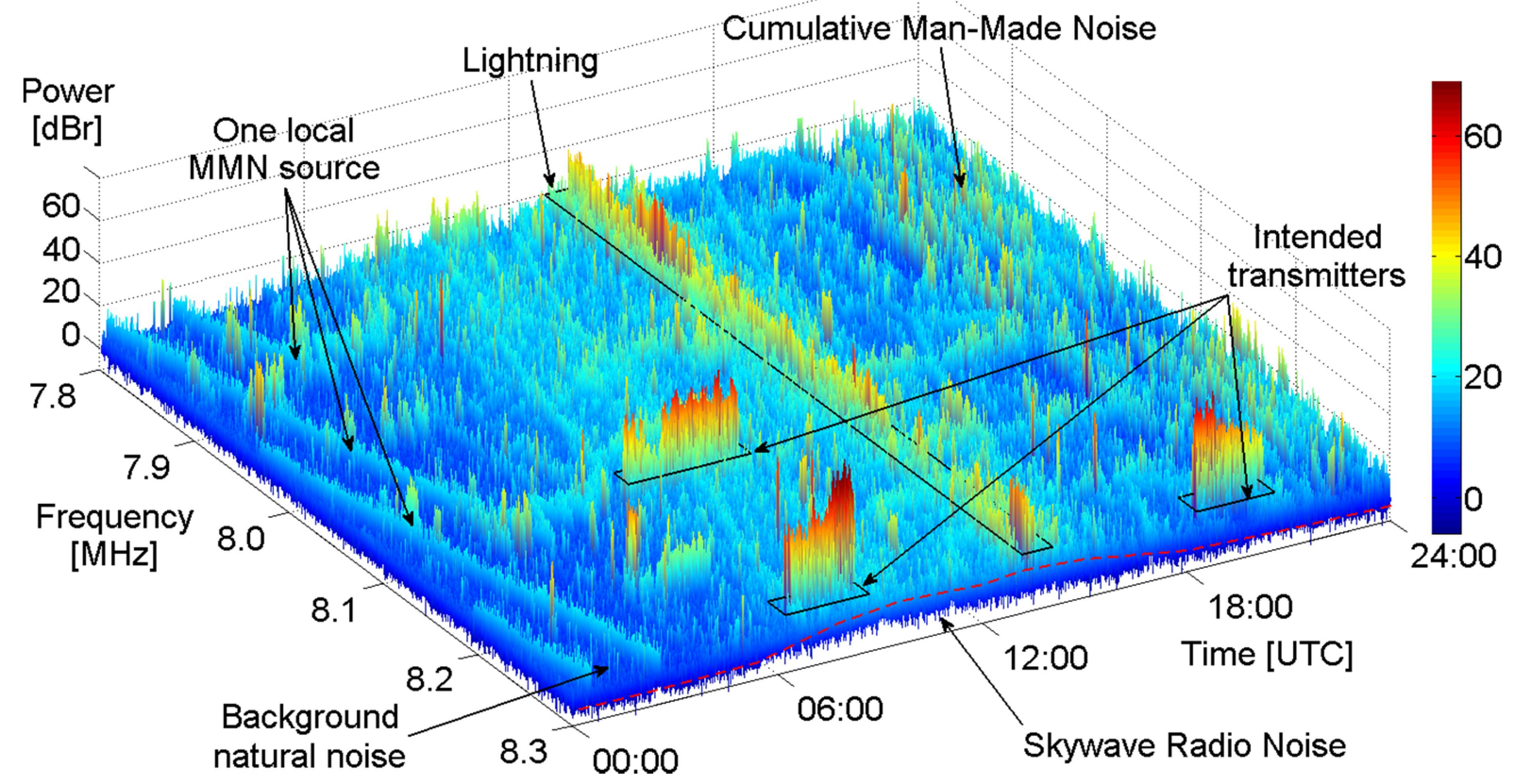

Figure 5.3 Measured diurnal variation of radio noise measured between 7.8 and $8.3 \mathrm{MHz}$ in a rural area. MMN stands for man-made noise. 


\subsection{A NOVEL METHOD FOR THE COMPARISON OF RADIO NOISE MEASUREMENT ANTENNAS}

As was shown in Section 5.2, the angular distribution and polarization of the incoming radio noise may vary with location and the origin of the noise. Therefore the ideal measurement antenna for radio noise measurements is equally sensitive to all spatial angles and all polarizations. Any selectivity of the measurement antenna with respect to polarization or spatial angle will create a bias towards a subset of the noise sources, which is undesirable. To evaluate this aspect of radio noise measurement antennas, two hemisphere graphs are proposed: one showing the antenna sensitivity regardless of polarization, the other showing polarization preference regardless of the absolute sensitivity. We will illustrate this method by evaluating a few radio noise measurement antennas, taken from [ITU-R Report SM.2055]. Only one operating frequency is used, wide-band properties of the antennas have not been evaluated.

\subsubsection{Short Lossless Vertical Monopole}

The first antenna to be examined is the SLVM, depicted in Figure 5.4. The antenna consists of a short vertical radiator, with a length $<<\lambda / 4$ on the operating frequency, fed against a radial network on or just below the ground [Thomas, 1950]. The antenna itself is lossy, but the ohmic losses are compensated for in the measurement results. The antenna pattern is calculated using NEC 4.2 [Burke, 2011] with ground parameters $\sigma=10 \mathrm{mS} / \mathrm{m}, \varepsilon_{\mathrm{r}}=17$ (farmland). The NEC 4.2 output file is processed to create the two hemisphere graphs. Figure 5.5 showing the hemisphere sensitivity graph, Figure 5.6 hemisphere polarization graph. The outskirt of the graph corresponds with the horizon, the center of the graph with the zenith. From Figure 5.5 we can see that the SLVM shows good coverage of the larger part of the hemisphere, except for a 10 to $20 \mathrm{~dB}$ reduction in sensitivity for elevation angles between $70^{\circ}$ and $90^{\circ}$. Figure 5.6 shows that the antenna is sensitive to vertical polarization only. Considering the radio noise propagation discussed in Section 5.2, the antenna is well suited for the measurement of man-made noise arriving from nearby sources at low elevation angles (Figure 5.1), but less suited for other noise components arriving at higher elevation angles or having a significant horizontal polarized component (Figure 5.2).

\subsubsection{Horizontal full-wave loop antenna}

A horizontal resonant square wire loop is a good HF high-angle radiator. Resonance is achieved when its overall length is between $1.03 \lambda$ and $1.06 \lambda$ long, depending on the height above ground. It may be fed in a corner or in the middle of one side; both methods produce the same radiation pattern. A drawing of the antenna is shown in Figure 5.7. Installed above farmland ( $\sigma=10 \mathrm{mS} / \mathrm{m}, \varepsilon r=17$ ), maximum radiation towards the zenith occurs when the antenna is $0.19 \lambda$ high. For better hemisphere coverage the antenna must be raised to $0.33 \lambda$. 


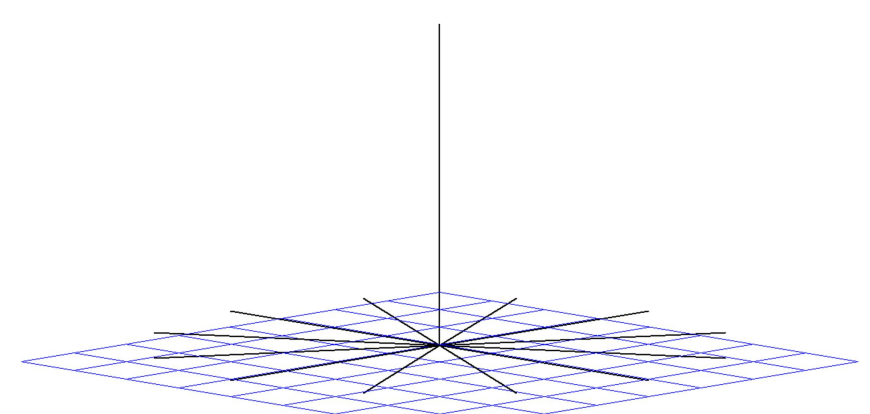

Figure 5.4 Short Lossless Vertical Monopole (SLVM), radiator length $<<\lambda / 4$.

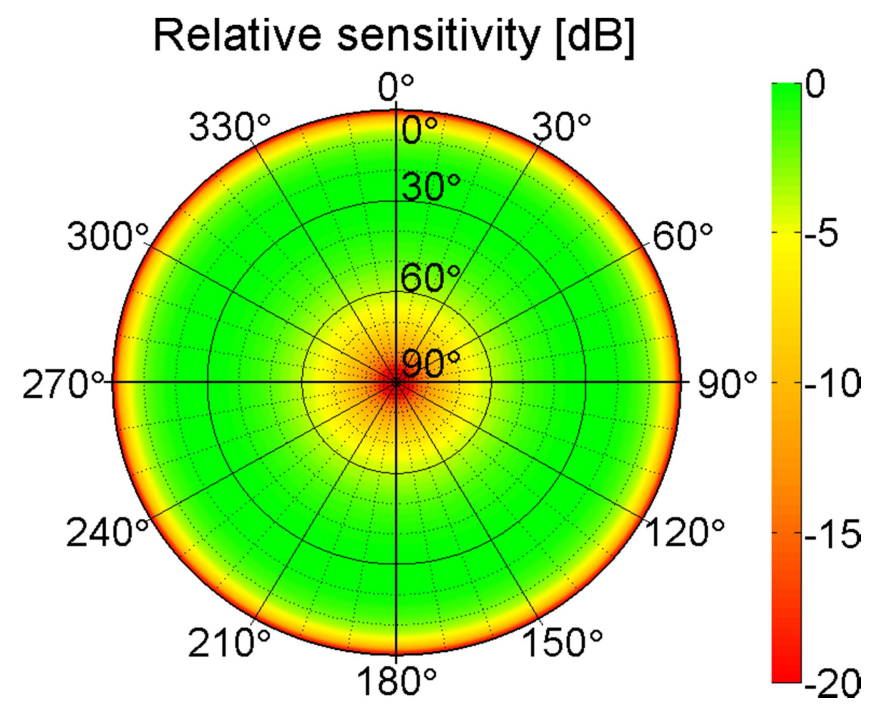

Figure 5.5 Hemisphere sensitivity graph of the SLVM. The antenna has reduced sensitivity at elevation angles above $60^{\circ}$.

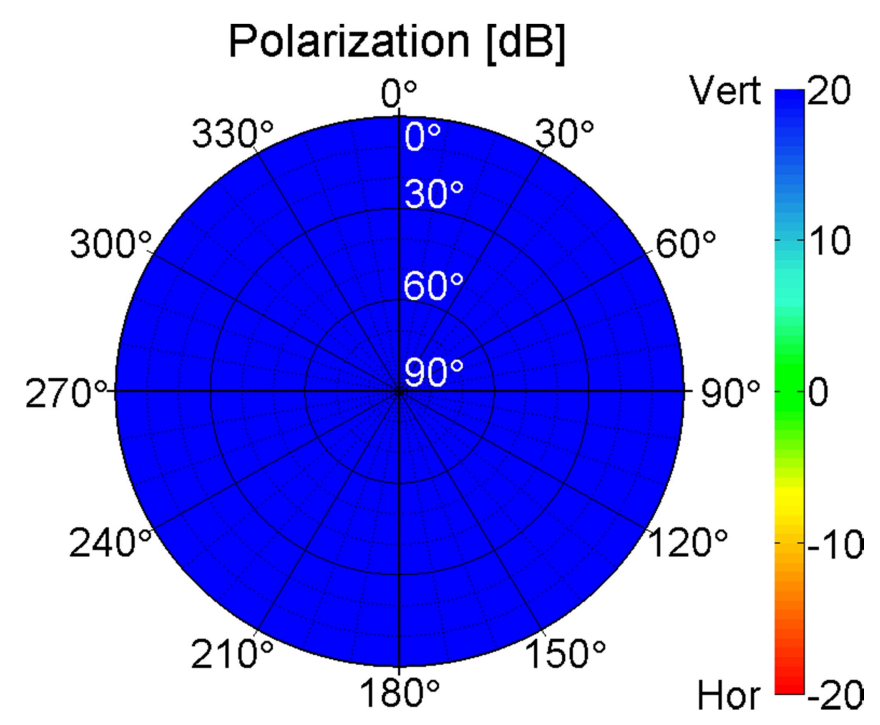

Figure 5.6 Hemisphere polarization graph of the SLVM. The antenna responds to vertical polarization only. 


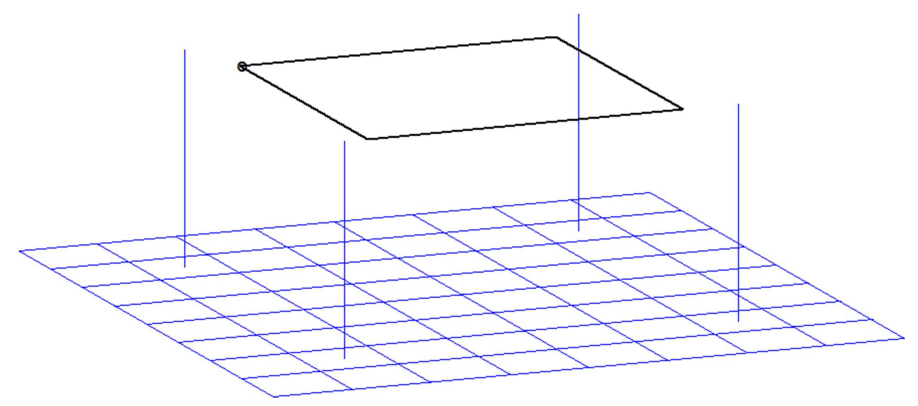

Figure 5.7 Horizontal full-wave loop antenna. Four supports are needed to suspend the antenna. Each side is approximately $0.26 \lambda$ long.

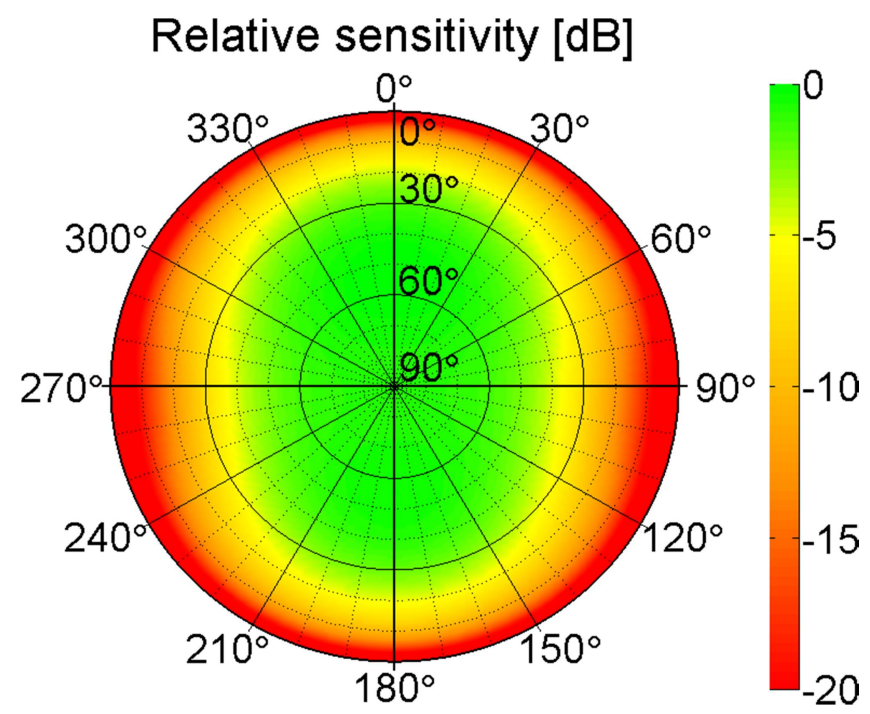

Figure 5.8 Hemisphere sensitivity graph of the horizontal full-wave loop antenna at a height of $0.33 \lambda$.

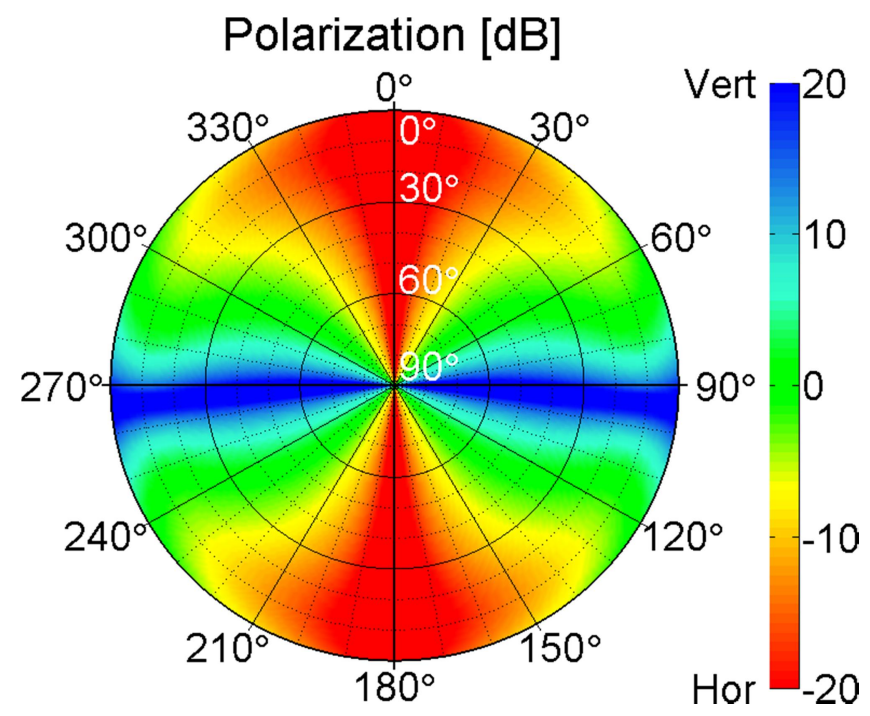

Figure 5.9 Hemisphere polarization graph of the horizontal full-wave loop antenna at a height of $0.33 \lambda$. Polarization varies with spatial angle. 
Whereas the radiation pattern of the SLVM is independent of frequency, the pattern of this antenna changes with frequency. Only the design frequency is considered in this analysis. Hemisphere sensitivity and polarization graphs are shown in Figures 5.8 and 5.9. Figure 5.8 shows a significant improvement in high elevation angle coverage over the SLVM, at the cost of some low angle sensitivity. As Figure 5.9 shows, polarization is no longer vertical, but it varies with the spatial angle of the incoming wave. The antenna needs 4 supports of $0.33 \lambda$ length, which corresponds with 14 meters at $7 \mathrm{MHz}$ and 33 meters at $3 \mathrm{MHz}$. This renders the antenna impractical for the lower HF frequencies.

\subsubsection{Inverted Vee half-wave dipole antenna}

Similar results may be achieved using an Inverted Vee half-wave dipole antenna, for which a single support suffices. Resonance is achieved when its overall length is between $0.47 \lambda$ and $0.49 \lambda$ long, depending on the apex height, end height and wire thickness. The antenna is depicted in Figure 5.10. When the antenna dimensions are chosen properly, the sensitivity diagram can be made almost circular. This can be seen in the hemisphere sensitivity diagram, Figure 5.11. For farmland soil, this is when the apex height is $0.21 \lambda$ and the end height is 0.05 $\lambda$. Resonant antenna length is $0.48 \lambda$ for a wire radius of $1 \mathrm{~mm}$. Similar to the horizontal fullwave loop antenna, the polarization of the Inverted Vee half-wave dipole antenna varies with the spatial angle of the incoming wave. The antenna shows horizontal polarization broadside to the antenna, and vertical polarization in the endfire direction, as can be seen in the hemisphere polarization diagram, Figure 5.12. This aspect is undesirable for radio noise measurements.

\subsection{UN-POLARIZED OMNI-DIRECTIONAL RADIO NOISE MEASUREMENT ANTENNA}

Figure 5.13 shows the antenna diagram for both polarizations at $70^{\circ}$ elevation angle, which shows that that the antenna response for horizontal and vertical polarization is very similar, but rotated $90^{\circ}$ in azimuth. When the dimensions specified in Figure 5.10 are observed, this is aspect is maintained for all elevation angles above $10^{\circ}$. Using this quality, an antenna system can be realized that has near-hemisphere coverage without bias towards horizontal vertical, left-hand or right-hand circular polarization. For this purpose, two orthogonal Inverted Vee half-wave dipole antennas are suspended from a single mast as depicted in Figure 5.14. Each antenna is connected to an identical measurement receiver. The noise power measured by each of the receivers is summed and stored. Figure 5.15 shows the resulting hemisphere sensitivity graph of this antenna. It has much better high elevation angle coverage than the SLVM, again at the cost of some low angle sensitivity. However, the hemisphere polarization graph, shown in Figure 5.16, shows that this antenna now responds equally well to all polarizations, regardless of the spatial angle, making this antenna system an interesting candidate for radio noise measurements that include the skywave component. A similar dual Inverted Vee antenna system was described in ITU-R Report SM.2055 [2006], but connected 
to a single receiver via a switch. And where ITU-R Report SM.2055 assumed that the performance of this set-up and that of the horizontal loop would be comparable, the new analysis method shows that this is not the case.

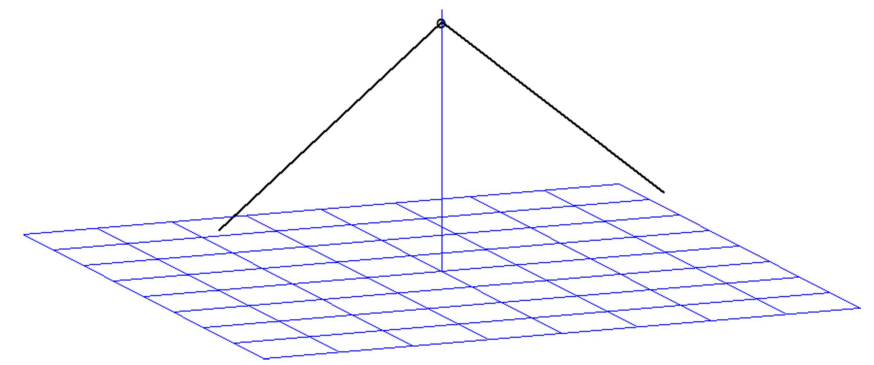

Figure 5.10 Inverted Vee half-wave dipole antenna, requiring only one support. Apex height is $0.21 \lambda$, end height is $0.05 \lambda$.

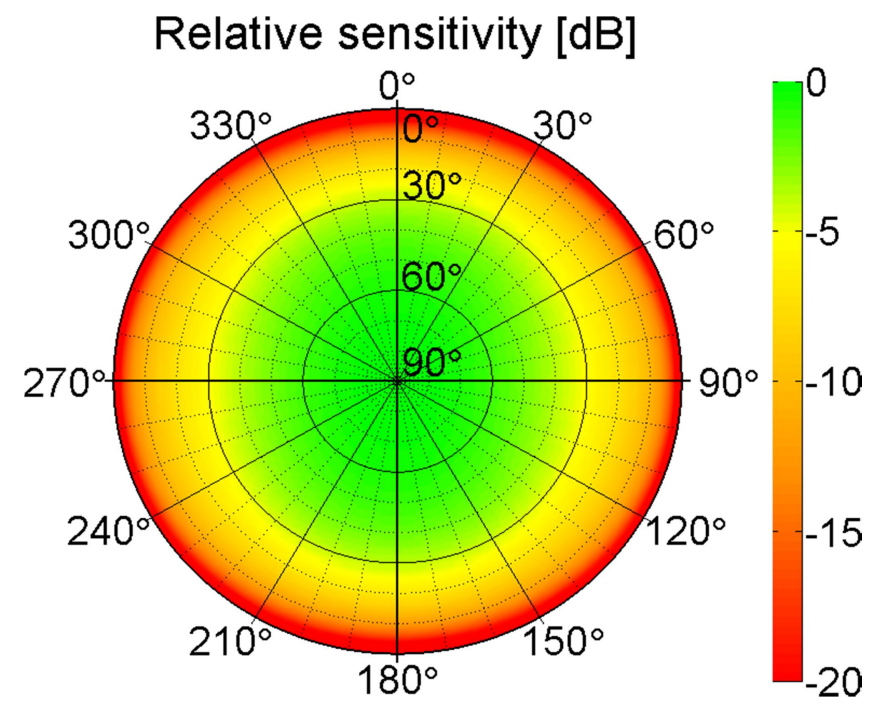

Figure 5.11 Hemisphere sensitivity graph of the Inverted Vee half-wave dipole antenna at a height of $0.21 \lambda$.

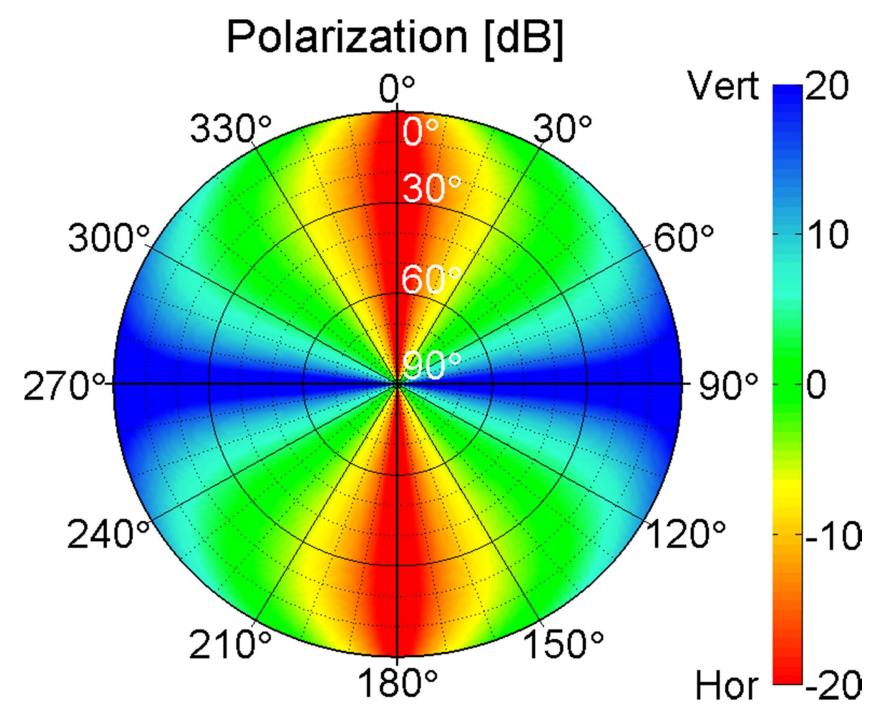

Figure 5.12 Hemisphere polarization graph of the Inverted Vee half-wave dipole antenna at a height of $0.21 \lambda$. 


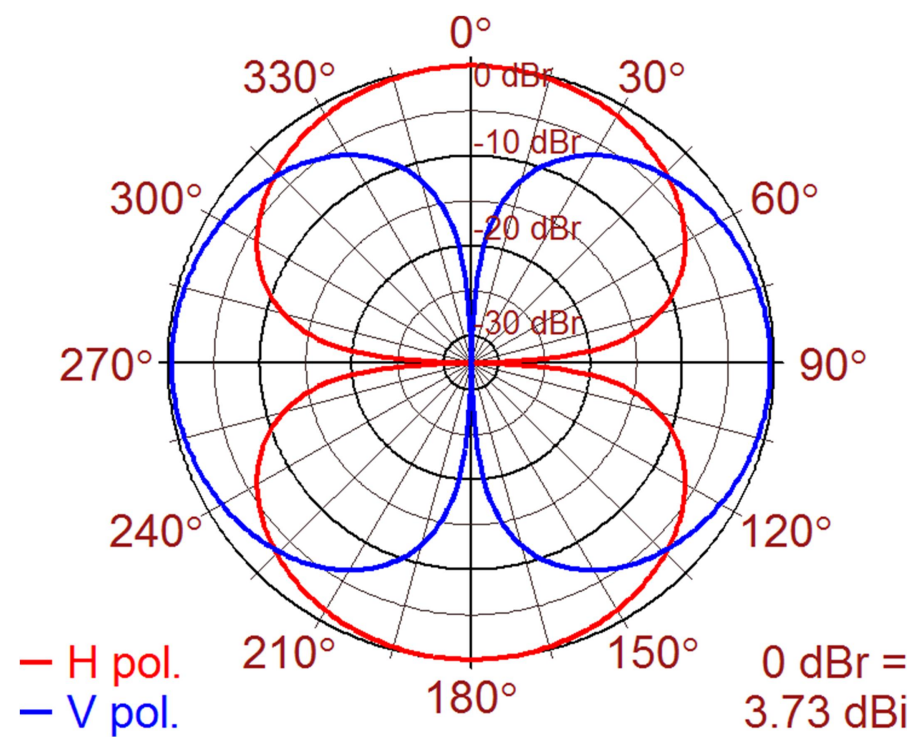

Figure 5.13 Horizontal antenna diagram of a single Inverted Vee half-wave dipole element for horizontal $(H)$ and vertical (V) polarization at $70^{\circ}$ elevation angle. Apex height is $0.21 \lambda$, end height is $0.05 \lambda$.

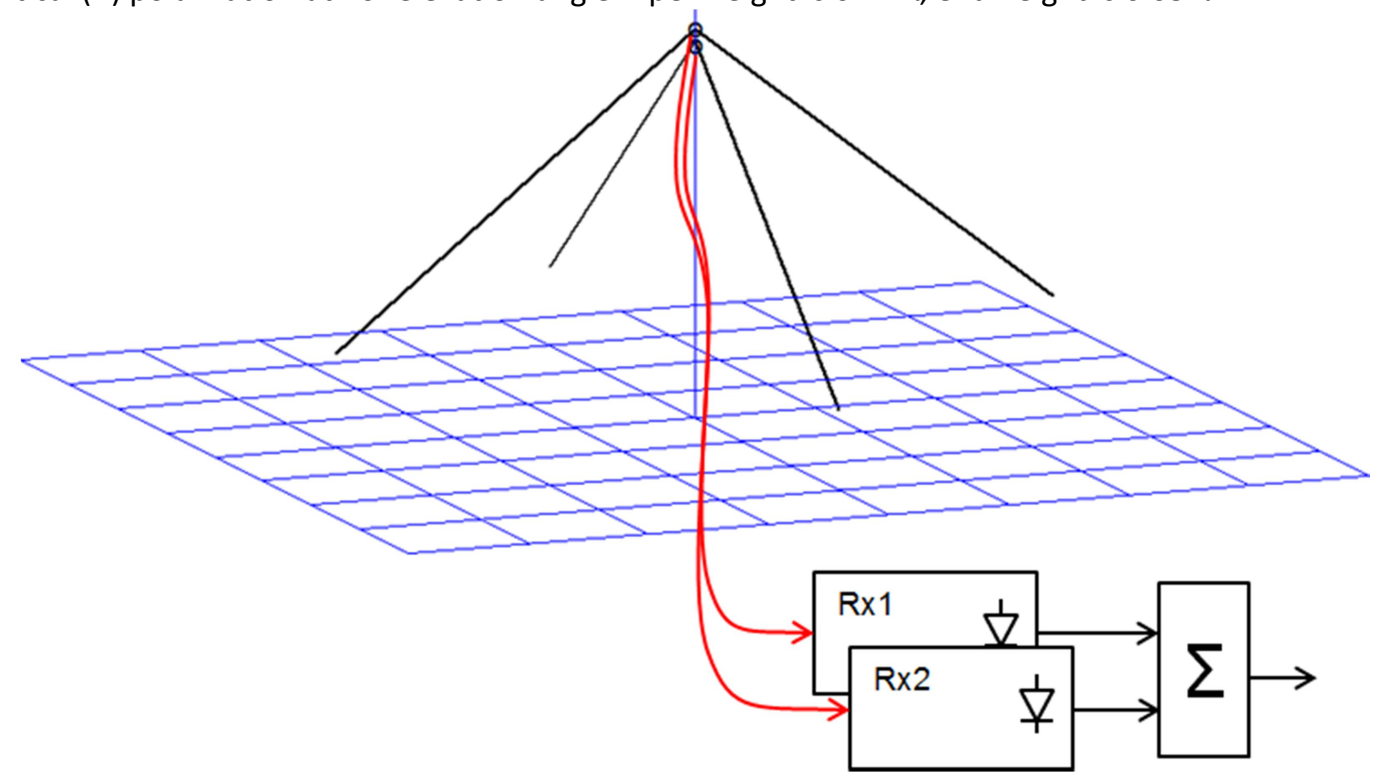

Figure 5.14 Un-polarized Omni-Directional Antenna System made of two orthogonal Inverted Vee half-wave dipole elements followed by two identical measurement receivers. The measured power is summed. 


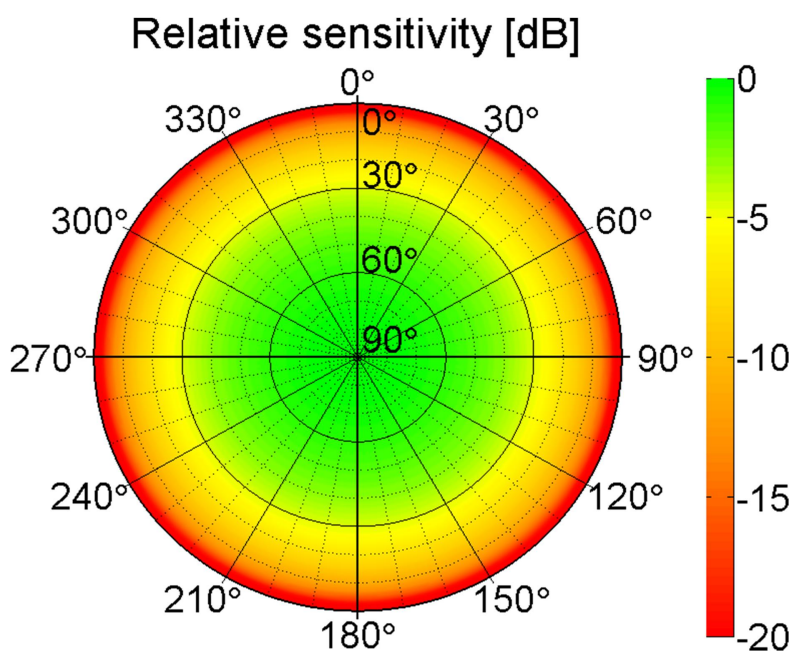

Figure 5.15 Hemisphere sensitivity graph of two orthogonal Inverted Vee half-wave dipole elements, followed by two identical synchronous measurement receivers.

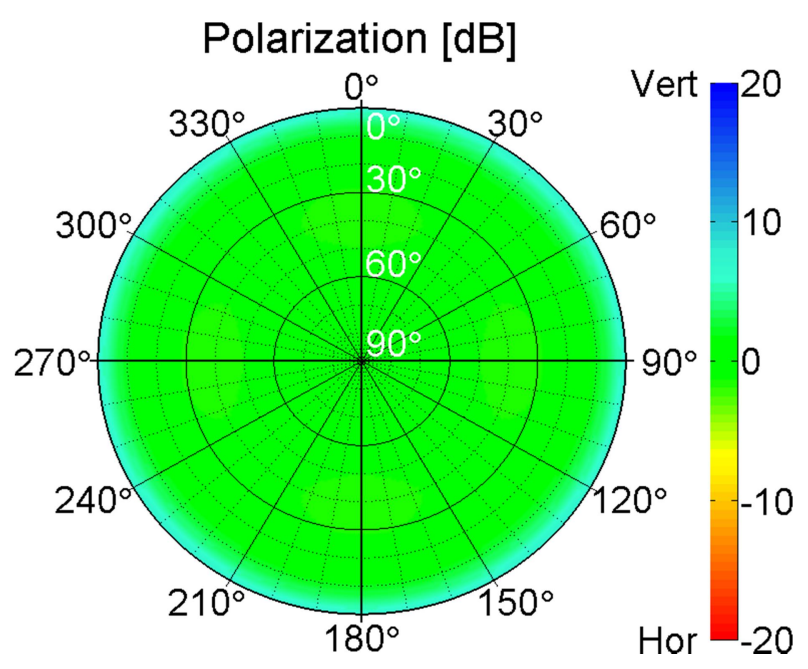

Figure 5.16 Hemisphere polarization graph of two orthogonal Inverted Vee half-wave dipole elements, followed by two identical synchronous measurement receivers.

\subsection{DISCUSSION}

A known problem of practical implementations of the SLVM is the coupling of the antenna to the lossy ground underneath the antenna. This is especially the case at lower frequencies, where the artificial ground plane in the design may be too small to effectively screen the antenna from the lossy ground below. This is an unwanted source of error. The original designs used in [Potter, 1932; Thomas, 1950] had large multi-wire ground screens to minimize this problem. The Inverted Vee antennas are much less affected by soil parameters.

For direct comparison of the antennas in a practical experiment only the difference in antenna efficiency has to be compensated for. Each antenna is intended to emulate an isotropic measurement antenna and their antenna factors would be identical if no losses 
were present. When efficiency differences are corrected for, direct comparison of the antennas becomes possible. Tests in different EMC environments, e.g. with predominantly ground wave noise or with a substantial skywave noise component, will show the spatial and polarizational preference of each antenna.

\subsection{CONCLUSION}

The novel method to compare radio noise measurement antennas has proven effective in identifying shortcomings and merits of HF radio noise measurement antennas. The search for an antenna which also covers skywave radio noise led to the optimization of an Inverted Vee Half-Wave Dipole antenna. To overcome the unfavorable polarization selectivity of this antenna, two such Inverted Vee Dipoles are suspended orthogonally from a single support and connected to two identical measurement receivers, resulting in an un-polarized, omnidirectional antenna for radio noise measurements. Stringent requirements regarding sensitivity and dynamic range need to be imposed on the HF measurement receiver used. However, suitable dual receiver platforms have become available recently through work of open source software defined radio design groups [HPSDR]. Storing coherent samples for each antenna allows reconstruction of the linear, left-hand and right-hand circularly polarized response. This enables new research options. The antenna solution presented was intended for single-frequency use, and the design must be modified slightly when other ground parameters prevail. Research is ongoing to make the antenna less dependent on ground constituency and to improve the low angle response of the antenna. Also a multi-frequency or wideband design is under study. 


\title{
6
}

\section{The importance of circular polarization for diversity reception and MIMO in NVIS propagation}

\begin{abstract}
Near Vertical Incidence Skywave propagation can provide High Frequency (HF: 3-30 MHz) radio coverage in an area of 300 by $300 \mathrm{~km}$ without the need for any intermediate infrastructure. It is therefore very well suited for disaster relief communication. However, electromagnetic waves refracted in the ionosphere are prone to fading caused by multipath propagation and by polarization change in the ionosphere. Historically, different diversity reception systems have been devised to reduce this fading. By contrast, modern HF Multiple Input Multiple Output (MIMO) techniques exploit the multipath aspects of the ionosphere to achieve an increased data throughput or a reduction of the required signal-to-noise ratio. The effectiveness of diversity reception and HF MIMO increases with decreasing correlation between the received signal streams. Electromagnetic waves propagating through the ionosphere consist of two characteristic wave components with circular polarization of opposite sense, the ordinary and extraordinary waves, each following a different path through the ionosphere. Adaptation of the polarization of the receive antennas to these characteristic waves provides two signal streams with high isolation. This is demonstrated with an experiment where both the ordinary and extraordinary wave are received separately. An isolation of at least $13 \mathrm{~dB}$ is shown.
\end{abstract}

Published as: B. A. Witvliet, E. van Maanen, G. J. Petersen, A. J. Westenberg, M. J. Bentum, C. H. Slump , R. Schiphorst, "The Importance of Circular Polarization for Diversity Reception and MIMO in NVIS Propagation," presented at European Conference on Antennas and Propagation, The Hague, The Netherlands, April 2015. 
Interaction of Antenna and Propagation Mechanism 


\subsection{INTRODUCTION}

Near Vertical Incident Skywave (NVIS) radio wave propagation uses the ionosphere as a reflector, on frequencies ranging from approximately 3 to $10 \mathrm{MHz}$. In NVIS, electromagnetic waves are launched at steep elevation angles, typically between $70^{\circ}$ and $90^{\circ}$, after which the waves are refracted in the ionosphere waves and land like droplets from a fountain in the area around the transmitter, as is depicted in Figure 6.1. A concise description of the NVIS propagation mechanism can be found in [Burgess, 1999].

NVIS is very well suited for disaster relief and military operations [Wallace, 1992], but it is also used widely in regions where other infrastructure is failing or non-existent. All ionospheric radio wave propagation is prone to 'fading', large and seemingly random short-term variations in received signal strength. This necessitates a large link margin to sustain the communication during the signal minima. Research into the reduction of the signal fading inherent to ionospheric radio propagation is almost as old as shortwave radio itself. This fading is partly caused by multipath propagation through the ionosphere in between the transmitter and the receiver and partly by the influence of electromagnetic wave polarization, as will be discussed. Variation in path length causes interference between the incoming waves arriving at the receiver via different paths, alternatingly causing additive or destructive interference.

In 1931 Beverage and Peterson observed [Beverage, 1931] large differences in instantaneous signal fading on widely spaced antennas and created a very effective spatial diversity system by adding the signals received on three widely space Fishbone antennas [Jasik, 1961]. As a delay between two ionospheric paths translates to a frequency dependent phase difference, multipath fading is frequency selective. Frequency diversity - using multiple signals with a few $\mathrm{kHz}$ separation - can be used to overcome this phenomenon.

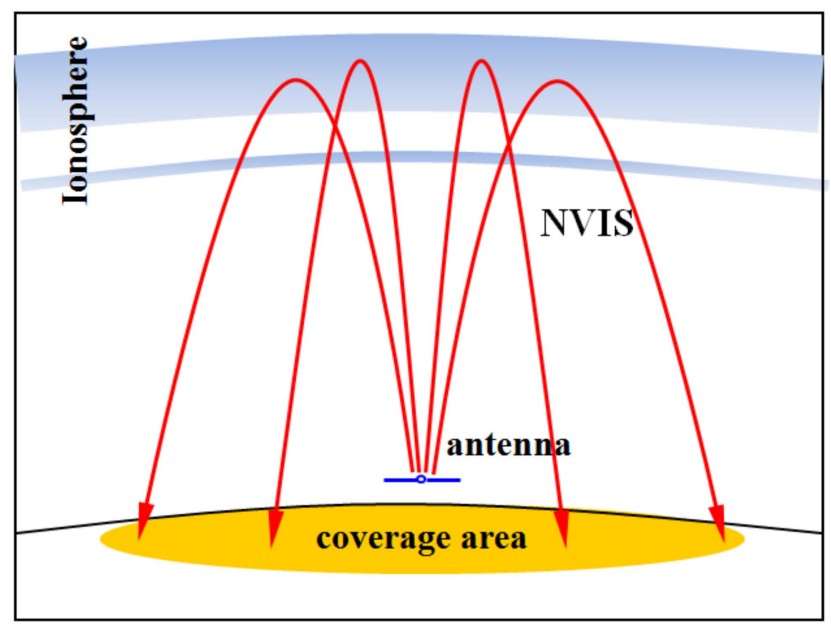

Figure 6.1 Near Vertical Incidence Skywave (NVIS) propagation: electromagnetic waves radiated nearly vertically are refracted in the ionosphere and land in an area directly around to the transmitter. 
This was already investigated in 1937 [Beverage, 1937] and forms the basis for the success of modern OFDM modems intended for HF use. Fading reduction using adaptive beam forming, by nulling out unwanted ionospheric modes, was demonstrated by Friis [1937]. The essence of these historic techniques, intended for intercontinental radio telegraph and radio telephone circuits, can still be found in modern HF radio communication systems, albeit in more elaborate forms.

\subsubsection{Circular polarization in NVIS propagation}

Appleton [1933] showed through experiments in 1931 that double echo's from the ionosphere actually consisted of a pair of characteristic waves reflected by the ionospheric layers. These characteristic waves, the ordinary and extraordinary wave, have circular polarization of opposite sense and experience different delays. For the Northern hemisphere, the extraordinary wave has the lesser delay and right hand circular polarization (RHCP), and the ordinary wave has the greater delay and left hand circular polarization (LHCP). At other instants only one echo was received with varying elliptical polarization. He explained his findings with his magneto-ionic theory in the same document. Varying mismatch between the polarization of the downward wave and that of the receive antenna, further indicated as 'polarization fading', multiplies with the multipath fading. Logically, polarization diversity was described in 1932 as another way to reduce fading [Runge, 1932].

\subsubsection{HF MIMO}

Only recently, in the last 10 years, ionospheric multipath propagation and polarization diversity are exploited to achieve an increase of data transfer speed or a decrease of necessary signal-to-noise ratio through HF Multiple Input Multiple Output (MIMO) technology. The benefits of MIMO are discussed in [Strangeways, 2006] with simulations of MIMO on HF propagation paths and measurements at $12.5 \mathrm{MHz}$ using narrowly spaced antennas. MIMO measurements over an $1800 \mathrm{~km}$ path between the Canary Islands and Spain [Perez-Alvarez, 2011] show the MIMO gain that can be achieved. High correlation factors were measured however, even with large antenna spacing. HF MIMO experiments in the United Kingdom using Near Vertical Incidence Skywave propagation (NVIS) on $6.2 \mathrm{MHz}$ are described in [Gunashekar, 2009]. Several antenna configurations are compared by measurement and mean correlation coefficients between 0.52-0.73 are established. Crossed dipoles are found to show less correlation than vertical antennas. The importance of the ordinary and extraordinary wave mode for HF MIMO is explained, but no experiments are performed with circularly polarized antennas. [Ndao, 2009] contains a feasibility study of an HF MIMO system and investigates also the role of the ordinary and the extraordinary wave, assuming matched polarization. However, this study is based on simulations and is not validated empirically. 


\subsubsection{Research question}

The effectiveness of both diversity reception and HF MIMO increases with decreasing correlation between the received signal streams. As mentioned above, electromagnetic waves propagating through the ionosphere consist of two characteristic wave components with circular polarization of opposite sense, each component following a different path through the ionosphere. If the natural decoupling between both characteristic waves is sufficiently large and if it can be put to use, this will drastically improve HF MIMO and diversity reception. To investigate this assumption, an experiment was designed and executed.

This chapter is structured as follows: Section 6.2 discusses the principles underlying the experiment. The practical realization of the experiment is described in paragraph 6.3. The measurement results and their interpretation are found in Section 6.4. The chapter concludes with the contributions made and lines for further research.

\subsection{EXPERIMENT DESIGN}

Under the influence of the earth' magnetic field an electromagnetic wave of linear polarization, when refracted in the ionosphere, continues its path as a composition of two circular waves with opposite sense, the characteristic waves. Their initial phase and amplitude difference is such that its sum represents the incoming linearly polarized wave. Their subsequent path through the ionosphere is different, as is shown in the example of Figure 6.2, which is produced using ionospheric ray tracing software [Solar Terrestrial Dispatch, 2010]. The delay and attenuation suffered by each wave is different as well. As a consequence, the polarization of the downward wave, generally a combination of both characteristic waves, is not identical to that of the original upward wave, but may have any polarization ranging from linear, via elliptical to circular, with an arbitrary orientation of the main polarization axis. Still, if the receive antenna would have circular polarization, only the power contained in the corresponding characteristic wave would be received.

As explained in [Burgess and Evans, 1999], the ionosphere supports NVIS radio wave propagation when the frequency of the electromagnetic wave does not exceed the critical frequency of the highest ionospheric layer. During daytime this is the F2-layer. The critical frequency of the F2-layer is slightly higher for the extraordinary wave (fxF2) than for the ordinary wave (foF2). An example of the variation of foF2 and fxF2 over the day is shown in Figure 6.3. For a given observation frequency, two intervals can be found where fxF2 exceeds the observation frequency while foF 2 is below the observation frequency. In these intervals pure circular polarization will be received. In Figure 6.3 these intervals are indicated with 'CP', the observation frequency is represented by a horizontal dashed line. 


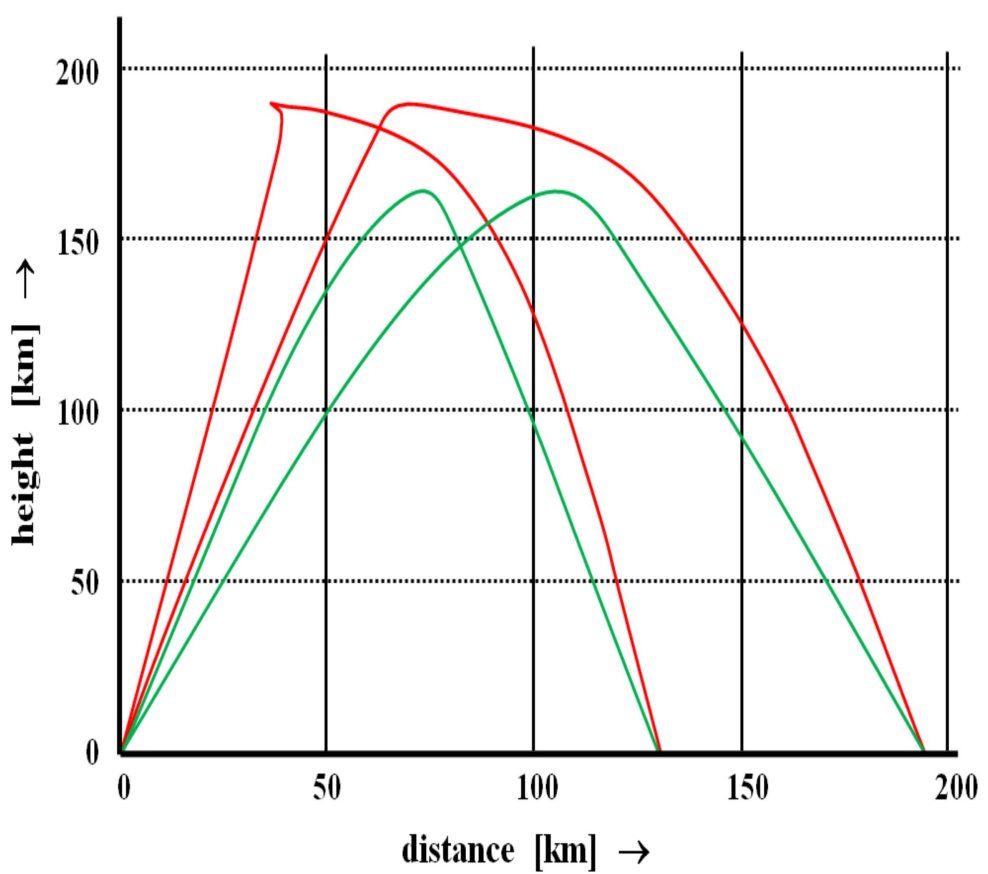

Figure 6.2 Ordinary (red) and extraordinary (green) waves follow different paths through the ionosphere. Simulated paths on $6.75 \mathrm{MHz}, 1$ October 2012 at 14:00z, at 52 ${ }^{\circ} \mathrm{N}, 6^{\circ} \mathrm{E}$ with SSN=75, using PropLab Pro v3.0.

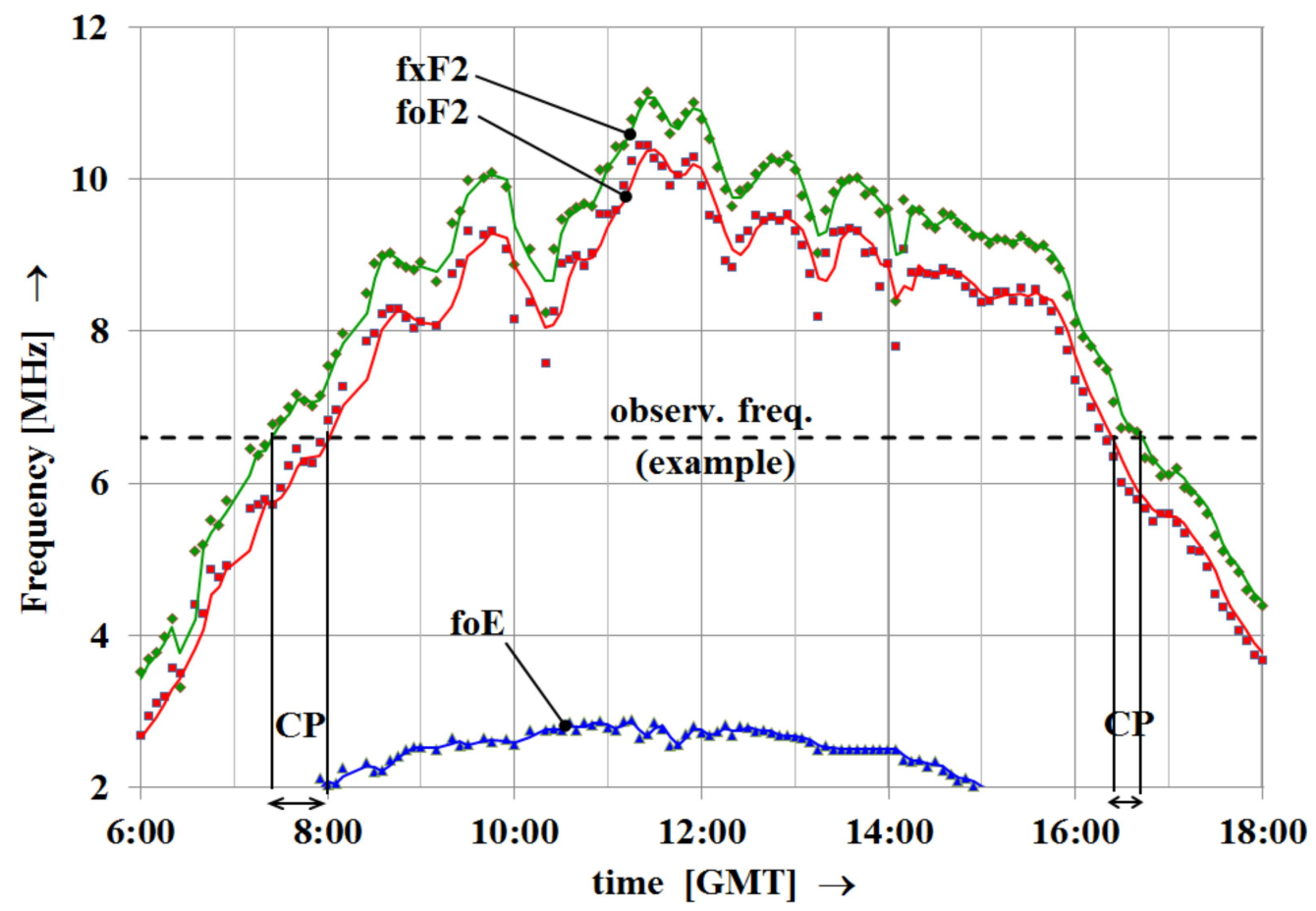

Figure 6.3 Variation of the critical frequencies of the F2-layer for the ordinary (foF2) and the extra-ordinary ( $f x F 2$ ) waves. An example is given of an observation frequency (dashed line) and two intervals in which circular polarization is received (CP). Data from the ionospheric sounder at Dourbes, Belgium of 12 Nov. 2012 is used. 
In the morning, when the electron density of the ionosphere increases under the influence of solar radiation, the extraordinary wave is the first to be refracted back to earth, only later to be followed by the ordinary wave. Inversely, when the electron density decreases again in the evening, the ordinary wave is the first to pass through the ionosphere, while the extraordinary wave is still refracted back to earth for some time. Therefore, NVIS propagation is expected to support the extraordinary wave during a slightly longer interval than it does the ordinary wave.

Also, just after NVIS propagation starts and just before it ends, a time interval exists in which the ordinary wave passes through the ionosphere, while the extraordinary wave is bent back to earth. In that time interval the electromagnetic wave coming down from the ionosphere will exhibit circular polarization, even when the transmit antenna has linear polarization. The situation around the magnetic poles and at the magnetic equator is different, but this is not described here for the sake of simplicity. Research in The Netherlands on the suppression of local man-made noise in short wave reception [Simons, 2006] reports circular polarization for NVIS signals on frequencies as low as $3.5 \mathrm{MHz}$, originating from transmitters with linear polarization. Triggered by these results, we decided to set-up an experiment to observe this phenomenon, to confirm the longer duration of the propagation interval for the extraordinary wave and measure the isolation between both characteristic modes during the intermediate interval at the start and end of the propagation interval.

The experiment is designed as follows: a linearly polarized electromagnetic wave is launched upwards towards the ionosphere by means of a stable beacon transmitter with a horizontal half wave dipole antenna. The antenna height is optimized for NVIS propagation [Burgess, 1999]. A frequency is selected on which a sufficiently long period of F-layer NVIS propagation is expected. This is done using sunspot cycle predictions of Hathaway [Hathaway] and ionograms of the nearest ionosonde located in Dourbes, Belgium [Royal Observatory of Belgium]. The occurrence of sporadic E-layer (Es) clouds is monitored closely using the same ionograms, so that a measurement date can be planned in which Es activity is low. At a distance, large enough to assure that the NVIS signal is much stronger than the ground wave signal, the measurement system is set-up. As earlier research has shown, a distance greater than approximately $50 \mathrm{~km}$ is sufficient at $6.8 \mathrm{MHz}$ [Burgess, 1999]. The measurement site has to be spacious and flat, with a homogeneous soil type and free of vegetation and man-made objects on and in the ground. At this measurement site an antenna is installed that can be switched electrically between left-hand (LHCP) and right-hand (RHCP) circular polarization. This antenna is connected to a calibrated professional measurement receiver. The received signal is measured alternatingly with LHCP and RHCP polarization and measurement values, switch position and timestamp are recorded for later processing. The practical realization of the experiment is described in Section 6.3, the measurements results are analyzed in Section 6.4 . 


\subsection{EXPERIMENT REALIZATION}

A stable beacon transmitter was set-up at $53.18278^{\circ}$ North, $6.30111^{\circ}$ East, producing a continuous wave RF signal with a power of 800 Watts into a horizontal half wave dipole antenna. Frequency used was $5.39 \mathrm{MHz}$, for which a special license had been obtained. The transmissions were switched in precisely timed one minute on, one minute off sequence, synchronized with the DCF77 time signal [Physikalisch-Technische Bundesanstalt]. The receive antenna was installed on a large grass land at $52.30833^{\circ}$ North, $6.10750^{\circ}$ East. Distance between transmitter and receiver was $99 \mathrm{~km}$, azimuthal direction was $187^{\circ}$, nearly due south. For the reception of circular polarization a Turnstile antenna [Kraus, 1988, pp. 726729] was realized with two perpendicular Inverted Vee half wave dipole antennas suspended from a single mast, as depicted in Figure 6.4. Dimensions of a single Inverted Vee antenna can be found in Figure 6.5 .

Circular polarization is produced by feeding the Inverted Vee antennas with a $90^{\circ}$ phase difference. The co-polar and cross-polar radiation patterns, calculated with $4 \mathrm{Nec} 2$ [Voors, 2012] using a NEC-4.2 core [Burke, 2011], are shown in Figures 6.6 and 6.7. If we assume perfect antenna balance and perfect quadrature feed the cross-polarization is greater than greater than $25 \mathrm{~dB}$ for NVIS angles. Phase errors and antenna asymmetry will reduce this value, thereby limiting the maximum measurable difference between both polarizations.

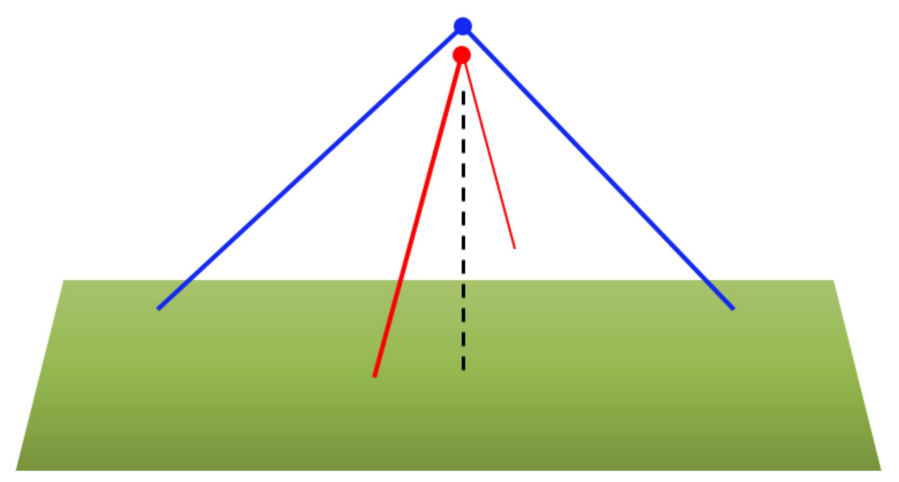

Figure 6.4 Turnstile antenna made of two perpendicular quadrature-fed Inverted Vee antennas.

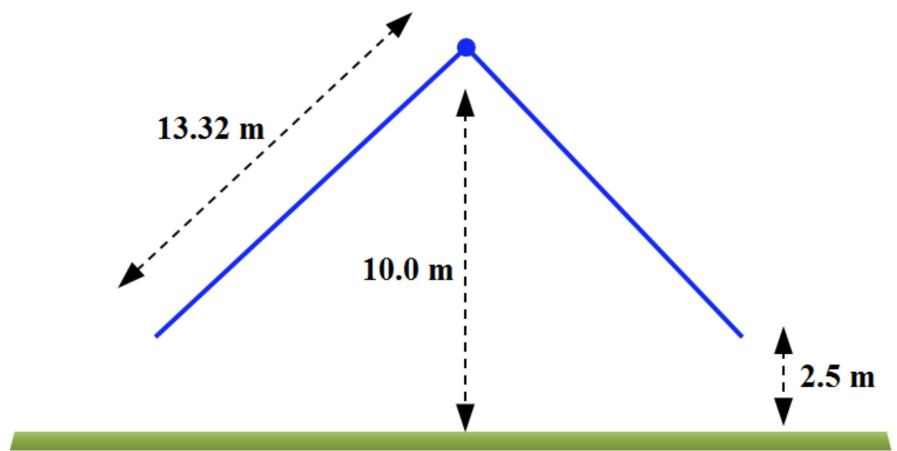

Figure 6.5 Approximate dimensions of the Inverted Vee antennas. 
Both antennas are connected to a coaxial feed line of identical length through a balance transformer. One feed line is extended with an electrical $\lambda / 4$ length of coaxial cable and the other with an electrical $\lambda / 2$ length of cable. The $\lambda / 2$ section can be bypassed using two coaxial relays, to obtain either $0^{\circ}$ or $180^{\circ}$ phase shift. This assembly produces a phase difference of $+90^{\circ}$ or $-90^{\circ}$ depending on the position of the coaxial relays, thereby producing RHCP or LHCP polarization. The schematic diagram of the phasing network is shown in Figure 6.8 .

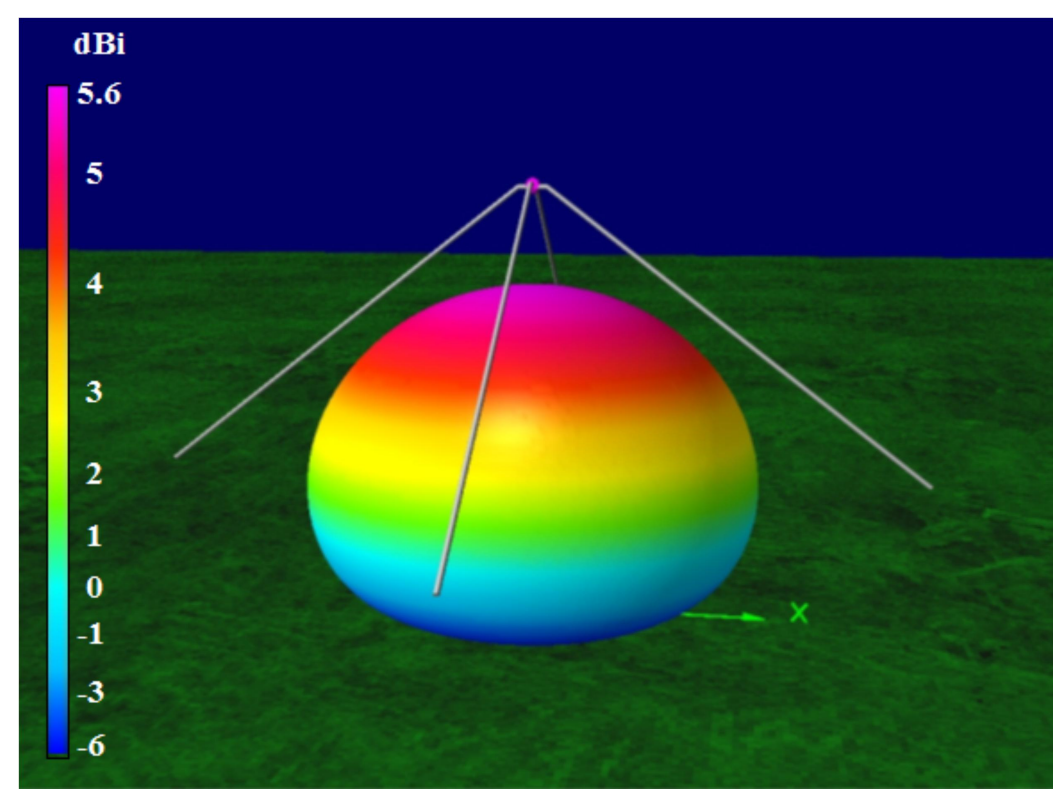

Figure 6.6 3D radiation pattern of the Turnstile antenna for matching circular polarization (co-polar). Maximum gain is $6.6 \mathrm{dBi}$. Image generated using $4 \mathrm{Nec} 2$ with a NEC-4.2 core.

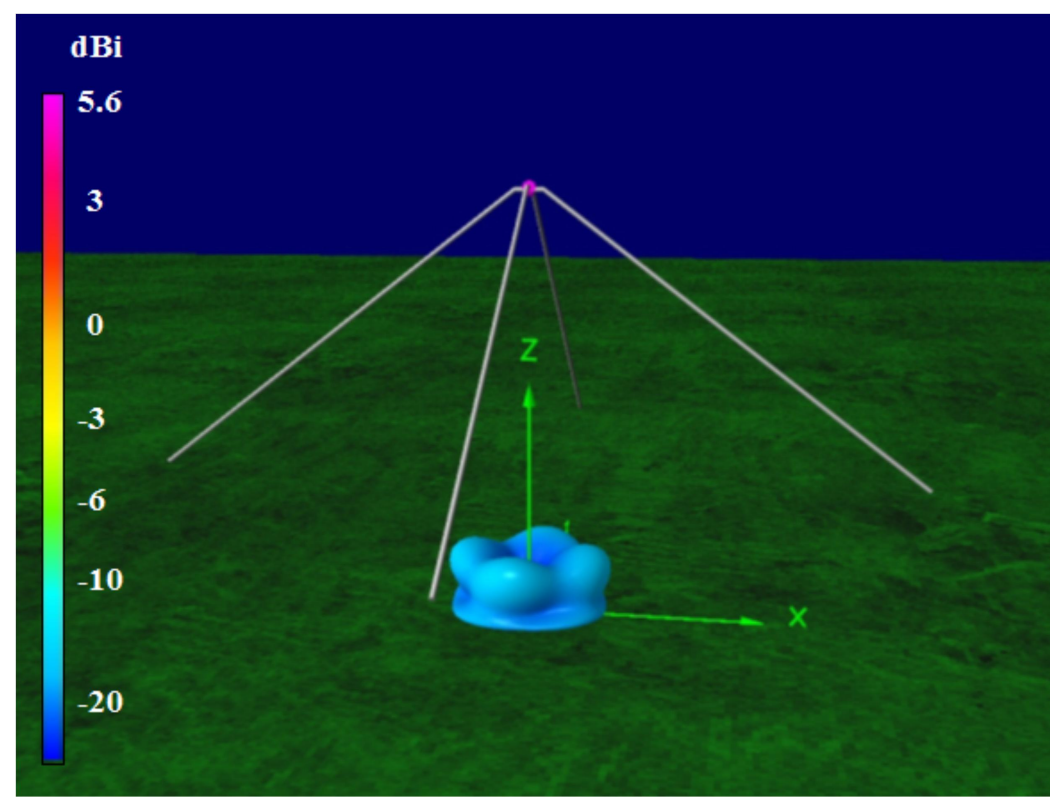

Figure 6.7 3D radiation pattern of the Turnstile antenna for opposite circular polarization. Cross polarization for NVIS angles $>25 \mathrm{~dB}$. Image generated using $4 \mathrm{Nec} 2$ with a NEC-4.2 core. 
The output of the power combiner is connected to a band pass filter, followed by a high dynamic range low noise amplifier (LNA), connected to a Rohde \& Schwarz FSMR26 measurement receiver. $A 3 \mathrm{~dB}$ attenuator inserted in between LNA and receiver perfects the output termination impedance of the LNA. Both the coaxial relays and the measurement receiver are controlled by a measurement program written in LabView running on a laptop computer. A block diagram is given in Figure 6.9. Signal strength values are measured alternatingly with LHCP and RHCP, and recorded together with a timestamp. Synchronization was again achieved using DCF77 time signals.

\subsection{MEASUREMENT RESULTS AND OBSERVATIONS}

The measurements were performed on 15 February 2009 from 08:00 to 13:00 GMT. The measured signal strength values for LHCP and RHCP reception are plot in Figure 6.10. Until t1 no NVIS propagation was possible. lonospheric scattering was observed, but signals were weak, approximately $25 \mathrm{~dB} \mu \mathrm{V}$, which is $35 \mathrm{~dB}$ above the ambient noise. After $\mathrm{t} 1$, the signal strength on the RHCP antenna rose approximately $32 \mathrm{~dB}$ in a few minutes time. The signal strength on the LHCP antenna followed suit, but remained approximately $13 \mathrm{~dB}$ lower.

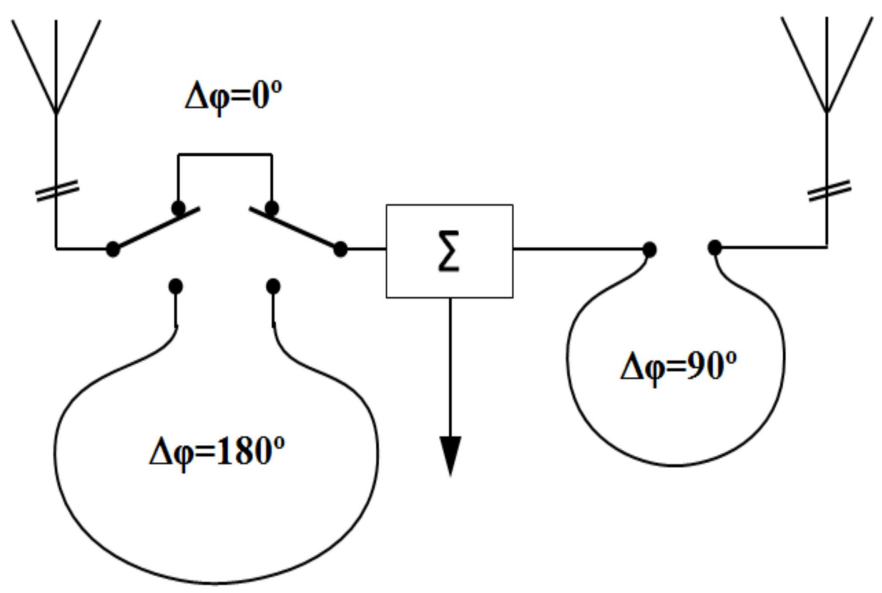

Figure 6.8 Switchable phasing network to obtain left-hand or right-hand circular polarization.

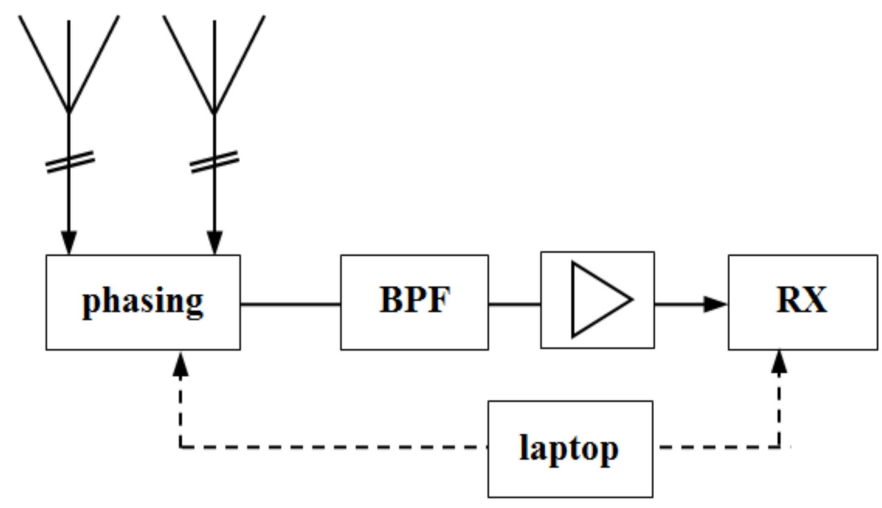

Figure. 6.9 Block diagram of the measurement setup. 


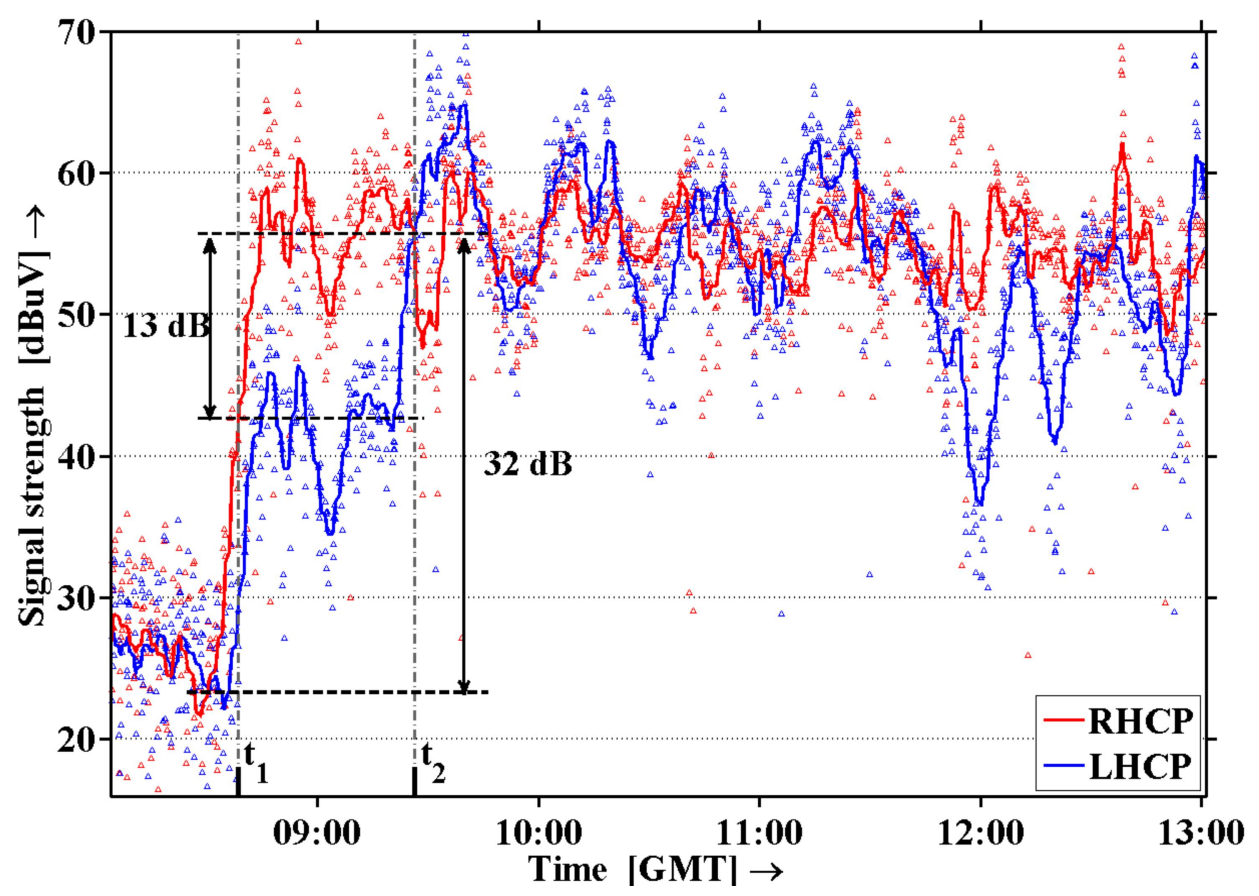

Figure 6.10 Measured LHCP and RHCP signal strength. Raw measurement samples are shown as dots. The continuous line shows the average signal strength within a sliding window of 4 minutes width.

This situation lasted for 45 minutes. At t2 the signal strength on the LHCP antenna rose further to attains approximately the same level as that measured on the RHCP antenna. Both antennas showed fading, but with different pattern and timing. The long-term slow fading was similar at times, and different at other instants. The short-term fading of the RHCP signal showed less pronounced excursions. These results show a measured isolation between LHCP and RHCP of at least $13 \mathrm{~dB}$. If we assume that the receive antenna (including the phasing network) provides a polarization discrimination that exceeds $13 \mathrm{~dB}$, this value corresponds with the isolation between the characteristic waves. If the cross-polarization of the receive antenna system is the limiting factor, the actual isolation between the characteristic waves is equal or greater than $13 \mathrm{~dB}$.

\subsection{CONCLUSIONS}

A switchable LHCP/RHCP antenna was used to measure the signal strength of a beacon transmitter emitting a linearly polarized wave, received via NVIS propagation. An isolation of at least $13 \mathrm{~dB}$ between the characteristic waves is measured. LHCP and RHCP signals show dissimilar fading. Fading is less pronounced on the RHCP antenna.

Considering the propagation mechanism involved and the apparent isolation between the ordinary and extraordinary wave, we may conclude that diversity reception will greatly benefit from the use of pairs of circularly polarized antennas with opposite sense. For HF 
MIMO, the use of LHCP and RHCP antennas at the transit side and the receive side will create two very well isolated channels, maximizing MIMO gain.

For two-way communication systems that have the capability of communicating the polarization received, switching to RHCP-only in the intermediate interval just after the start of the NVIS propagation window and just before closure of that window will increase the effectively used power by $3 \mathrm{~dB}$ in those intervals. Additionally, switching to RHCP-only at the receiver will increase the signal-to-noise ratio by approximately $3 \mathrm{~dB}$, resulting in a system gain of $6 \mathrm{~dB}$. For Single Input Single Output (SISO) systems, replacing linearly polarized antennas by antennas with circular polarization will strongly reduce multipath caused by interference between both characteristic waves. 


\title{
Measuring the isolation of the circularly polarized characteristic waves in NVIS propagation
}

\begin{abstract}
Separate excitation of the characteristic waves in the ionosphere results in two orthogonal propagation channels on the same frequency, which may be used in diversity and Multiple Input Multiple Output (MIMO) systems. In this chapter a method to measure the isolation between these paths is proposed and demonstrated in a Near Vertical Incidence Skywave (NVIS) experiment on a frequency of $7 \mathrm{MHz}$ over a $105 \mathrm{~km}$ distance. Characteristic wave isolation exceeding $25 \mathrm{~dB}$ is measured during 'Happy Hour': the interval when the propagation path just opens or closes and only the extraordinary wave propagates.
\end{abstract}

Published as: B. A. Witvliet, E. van Maanen, G. J. Petersen, A. J. Westenberg, M. J. Bentum, C. H. Slump, R. Schiphorst, "Measuring the Isolation of the Circularly Polarized Characteristic Waves in NVIS Propagation," in IEEE Antennas and Propagation Magazine, June 2015. 
Interaction of Antenna and Propagation Mechanism 


\subsection{INTRODUCTION}

Ionospheric radio wave propagation can be used to bridge hundreds of kilometers with a direct radio link [Davies, 1990]. This makes ionospheric radio communication valuable when independence of satellite or terrestrial networks is desired, e.g. in regions without telecommunication infrastructure [Hervás, 2013], for disaster relief operations in areas where the telecommunication infrastructure is destroyed [Kwasinski, 2006; Tang, 2006; Kobayashi, 2014] and for defense operations [Fiedler, 1996]. When the frequency is properly chosen - typically between 3 and $10 \mathrm{MHz}$ - radio waves sent upwards are reflected by the ionosphere to create a large continuous coverage area (400 by $400 \mathrm{~km}$ ) around the transmitter. As was shown in Chapter 4, the antenna system has to concentrate the transmit power at high elevation angles, typically $70-90^{\circ}$, hence the name of the propagation mechanism: Near Vertical Incidence Skywave (NVIS). A simplified illustration of the NVIS concept is given in Figure 7.1, the propagation mechanism is described in detail in [Fiedler, 1996].

Ionospheric radio wave propagation adds fading to the received signal, decreasing the link reliability and throughput, but this may be countered with diversity reception or MIMO [Strangeways, 2006]. Improved diversity reception can be obtained by adapting the polarization of the antenna to the circular polarization of the characteristic waves propagating in the ionosphere, thereby creating two independent propagation paths from transmitter to receiver as was discussed in Chapter 6. In this chapter a method to measure the isolation between these paths is proposed, and subsequently demonstrated in an NVIS experiment on a frequency of $7 \mathrm{MHz}$ over $105 \mathrm{~km}$ distance. The 'Happy Hour' propagation phenomenon that facilitates this measurement is described, as well as the required equipment and its calibration and accuracy.

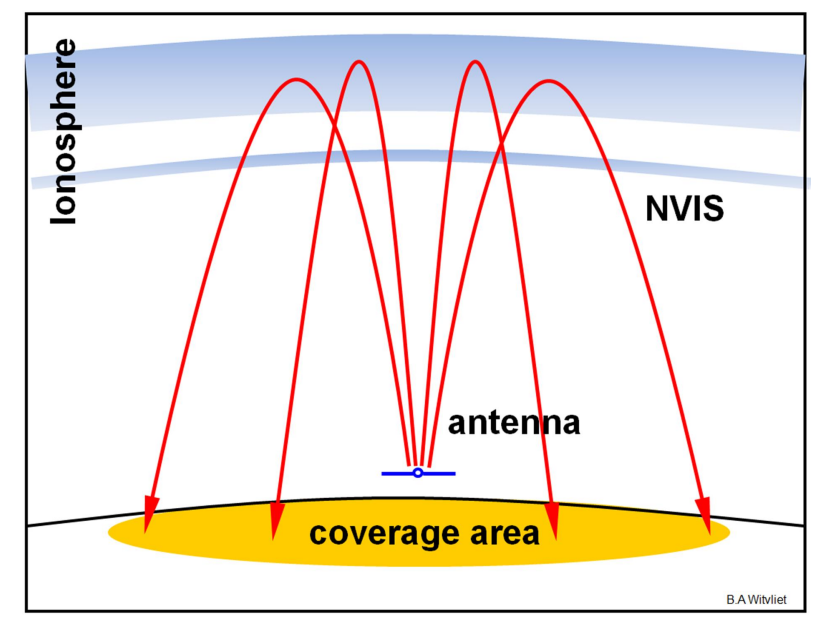

Figure 7.1 Near Vertical Incidence Skywave (NVIS). lonospheric radio wave propagation can be used to cover a continuous area with a radius of several hundred kilometers, using a single transmitter. Frequencies between 3 and $10 \mathrm{MHz}$ are used and the radio waves must be radiated at steep angles. 
This chapter is structured as follows. Section 7.2 discusses NVIS characteristic wave propagation and explains the Happy Hour phenomenon. In Section 7.3 an experiment is proposed to exploit this phenomenon for characteristic wave isolation measurements. The equipment used is described in detail. In Section 7.4 the measurement results are analyzed, followed by a discussion in Section 7.5 and conclusions in Section 7.6.

\subsection{CHARACTERISTIC WAVES IN NVIS PROPAGATION}

In 1932, Appleton and Builder [Appleton, 1932] discovered that electromagnetic pulses sent up towards the ionosphere were received as pulse-pairs at a distance of $5 \mathrm{~km}$ from the transmitter. One of their registrations is reproduced in Figure 7.2. The transmit pulse is first received via ground wave (G), then twice via F1-layer reflection (F1' and F1") and twice via F2-layer reflection (F2' and F2"). Appleton explained this phenomenon with his magnetoionic theory [Appleton, 1933], showing that under the influence of the earth' magnetic field an electromagnetic wave of arbitrary polarization is split into two circularly polarized waves of opposite sense when entering the ionosphere. Ratcliffe [1962] showed that only these waves propagate in the ionosphere, and named them 'characteristic waves'. Each characteristic wave follows its own path and experiences a particular (variable) attenuation and delay. The 'ordinary wave' follows a path similar to the path that it would have followed when no magnetic field had been present. The other characteristic wave is named the 'extraordinary wave'. In the Northern hemisphere the downward ordinary wave has lefthand circular polarization and the greater delay, the extraordinary wave has right-hand circular polarization and the lesser delay.

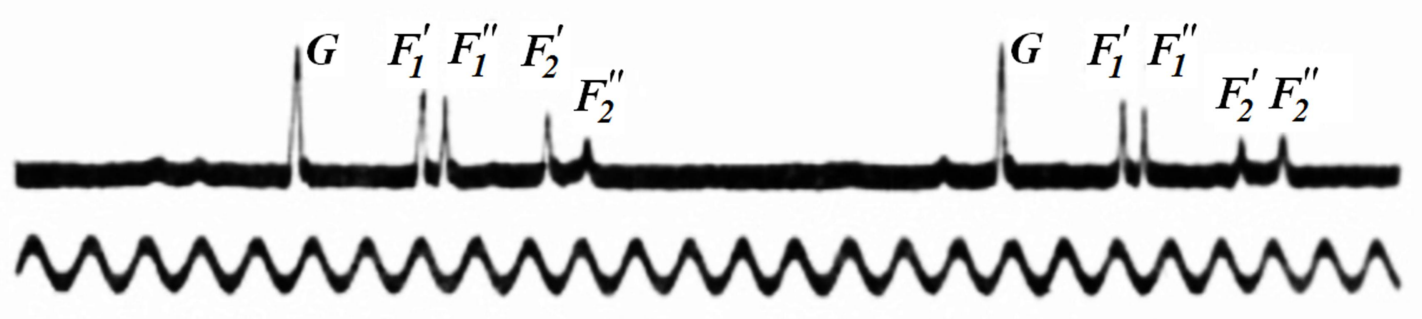

Figure 7.2 Pulse delay measurements by Appleton and Builder. Figure reproduced from [Appleton, 1932]. Upper trace: transmitted pulses are first received via ground wave (G), then twice via the F1-layer (F1' and F1") and twice via the F2-layer (F2' and F2"). Lower trace shows a $1115 \mathrm{~Hz}$ sine wave, serving as time reference. 


\subsubsection{The Happy Hour propagation interval}

Figures 7.3 through 7.10 show the simulated paths of the ordinary (in red) and the extraordinary wave (in green) through the ionosphere, over a horizontal distance of approximately $90-120 \mathrm{~km}$. The reflection takes place in the F2 layer at $180-280 \mathrm{~km}$ height, the reflection height and path geometry vary over the day. PropLab Pro 3 ionospheric ray tracing software [Solar Terrestrial Dispatch, 2010] is used, simulations are made at a frequency of $7 \mathrm{MHz}$ on 9 March 2014 with a Smoothed Sunspot Number (SSN) of 79 and an effective sunspot number (IGN) of 164. In 24 hours, the propagation varies as follows:

At night, the electron density of the ionosphere is too low to support NVIS propagation at the selected operating frequency and both waves pass through the ionosphere (Figure 7.3). In the morning, the radiation of the sun causes a steep rise in the electron density of the ionosphere, until it is sufficiently high to reflect the extraordinary wave at the operating frequency. However, the ordinary wave still passes through the ionosphere (Figure 7.4). The received signal consists of the extraordinary wave only and is circularly polarized. This situation remains for some time, until the electron density has increased sufficiently for the ordinary wave to reflect as well (Figure 7.5). Now the received signal is a summation of the ordinary and the extraordinary wave components. As each varies in strength and delay, the received signal shows rapidly changing polarization.

Throughout the day, both waves propagate from transmitter to receiver. Their path height has lowered due to a further increase in electron density (Figures 7.6 and 7.7). In the evening the electron density slowly decreases again and the path altitude increases again (Figure 7.8). At a certain instant the electron density becomes too low to reflect the ordinary wave and it passes through the ionosphere, while the extraordinary wave is still reflected (Figure 7.9). Again, the received signal consists of the extraordinary wave only and is circularly polarized. This situation remains for some time, until the electron density has decreased so much that the extraordinary wave passes through the ionosphere also (Figure 7.10). This situation remains until the solar radiation increases the ionization again the next morning.

We identified two exceptional intervals (Figures 7.4 and 7.9) which we nicknamed 'Happy Hour', in which only the extraordinary wave propagates and right hand circular polarization (RHCP) is received. This phenomenon was predicted (but not observed) in [McNamara, 2008] and experimentally confirmed in Chapter 6. At sunrise the ionization shows a steep gradient and consequently the morning Happy Hour is short, typically 30 minutes at mid-latitudes in winter. The evening Happy Hour often lasts more than an hour, due to the slower recombination processes. 


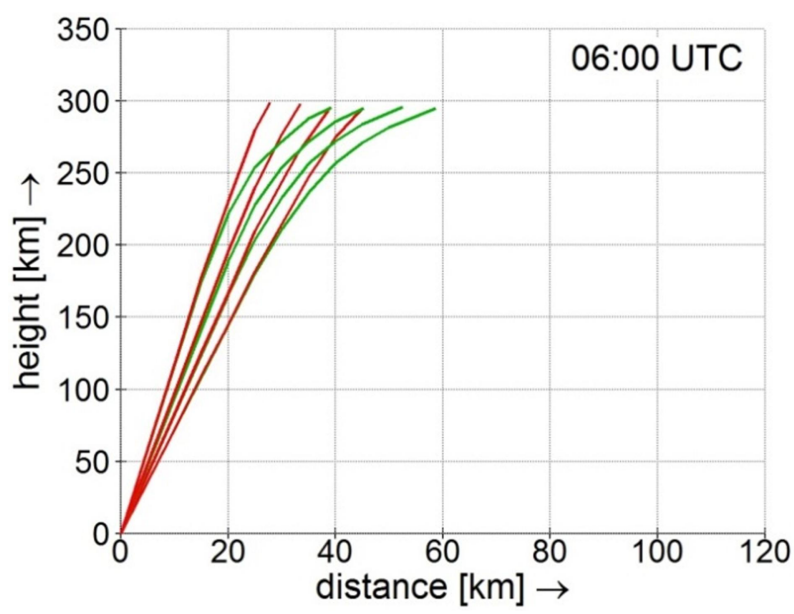

Figure 7.3 Ionospheric paths of the ordinary wave (red) and the extraordinary wave (green) at 06:00 UTC. Daylight is from 06:02 to 16:28 UTC. The ionization of the ionosphere is not sufficient to reflect either of the characteristic waves. Simulation parameters, see text.

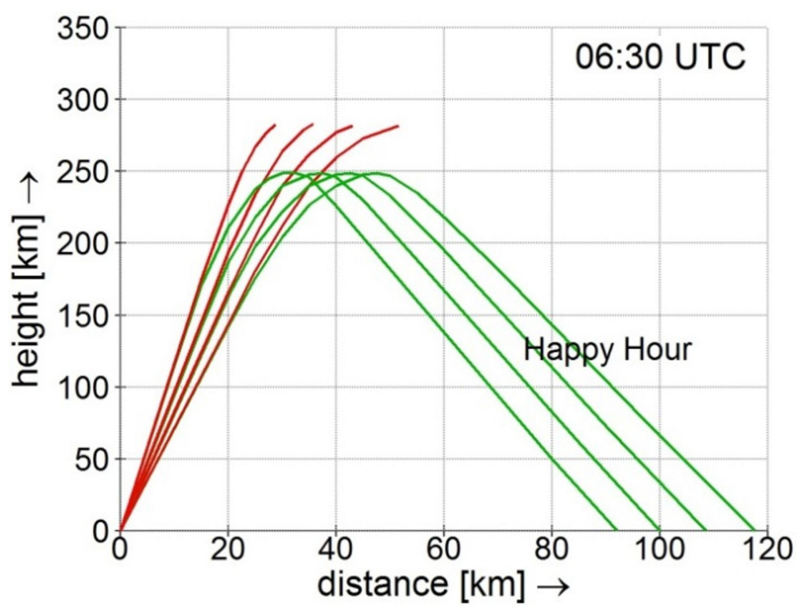

Figure 7.4 At 06:30 UTC the ionization of the ionosphere has sufficiently increased to reflect the extraordinary wave (green), the ordinary wave (red) is not reflected yet. The downward wave has circular polarization. This is the morning Happy Hour.

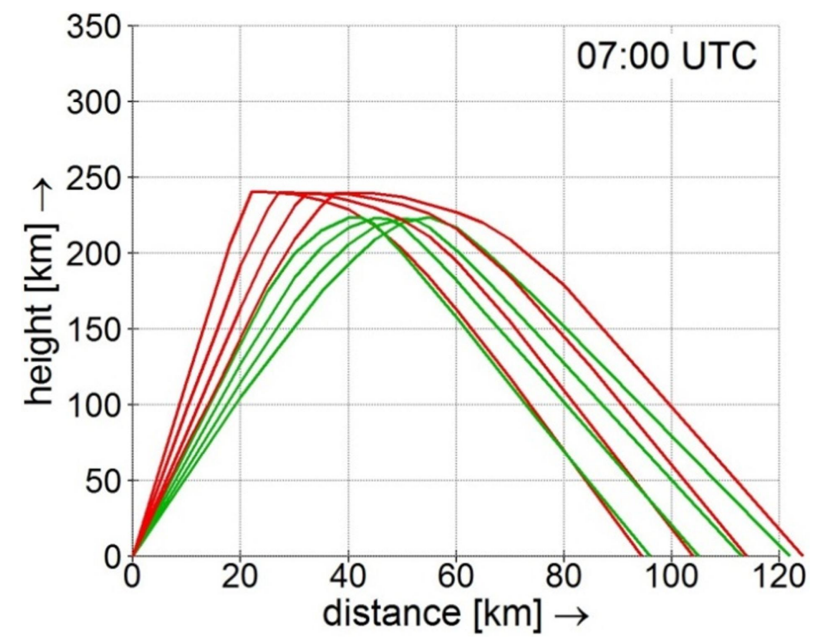

Figure 7.5 At 07:00 UTC both the ordinary and the extraordinary wave are reflected. Received polarization is highly variable. 


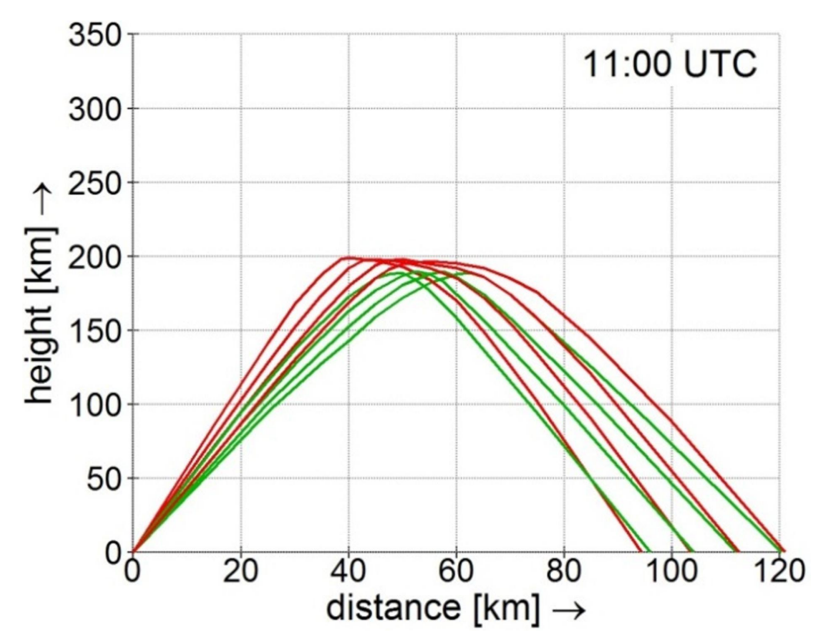

Figure 7.6 At 11:00 UTC the reflection height is lowered due to the increased electron density of the ionosphere.

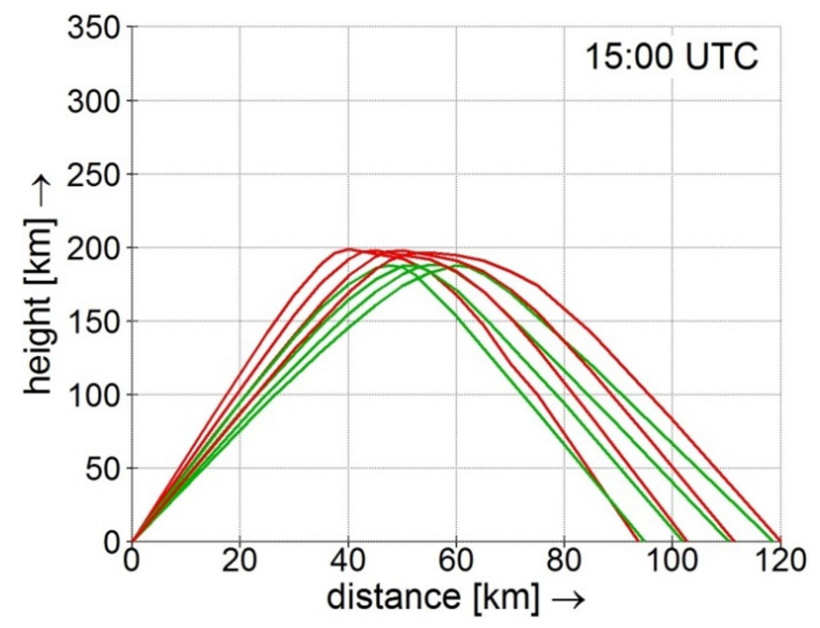

Figure 7.7 The propagation of both waves continues at 15:00 UTC.

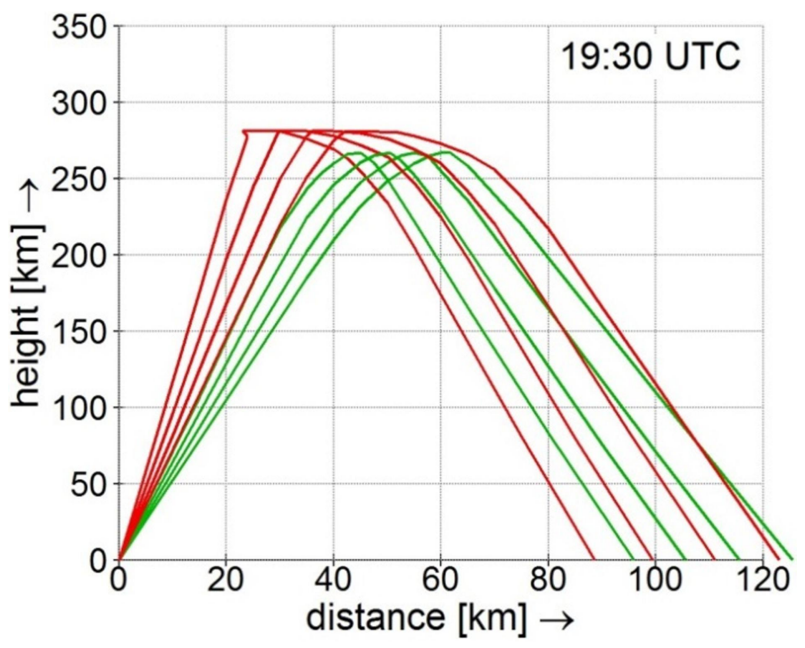

Figure 7.8 After 16:38 UTC (sunset) the ionization decreases. At 19:30 UTC waves already penetrate much further into the ionosphere: the reflection height increases. 


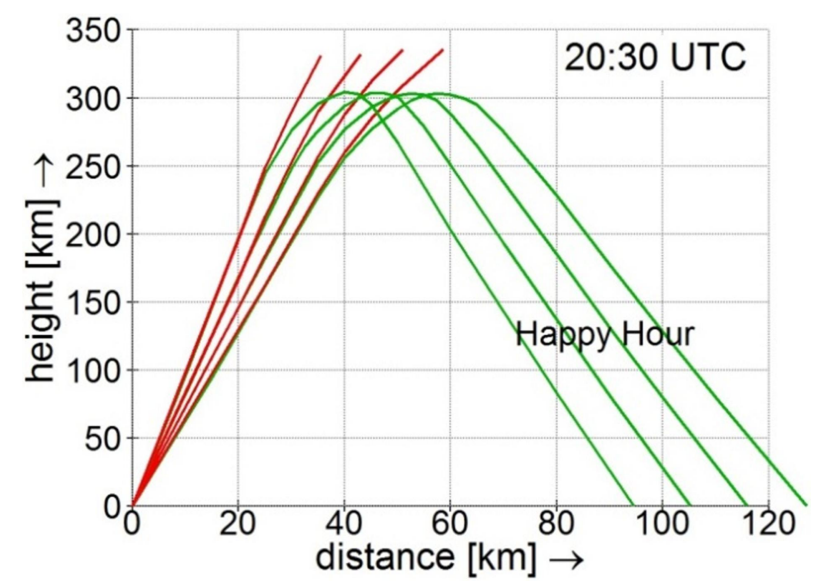

Figure 7.9 At 20:30 UTC the electron density of the ionosphere has decreased so much that the ordinary way is no longer reflected, but the extraordinary wave is supported still. The downward wave has circular polarization. This is the evening Happy Hour.

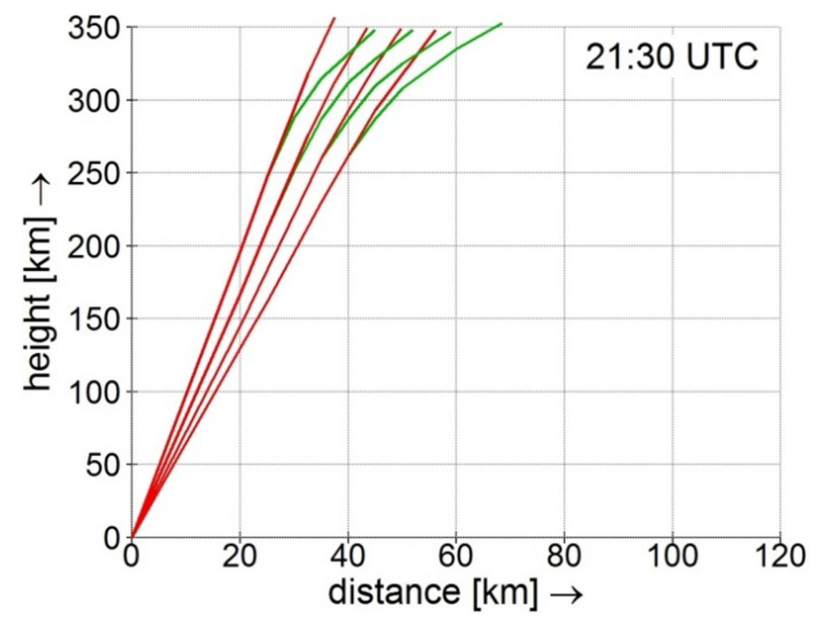

Figure 7.10 At 21:30 UTC the ionization of the ionosphere has decreased so much that none of the characteristic waves is reflected anymore.

\subsection{MEASURING NVIS CHARACTERISTIC WAVE ISOLATION}

This propagation phenomenon can be used to measure the isolation between the ordinary and the extraordinary wave. If substantial isolation between the RHCP and LHCP waves can be demonstrated in the Happy Hour intervals, this will provide a strong support for the assumption that the characteristic waves travel independent paths through the ionosphere with little crosstalk, and that they can be used effectively in NVIS diversity and MIMO. We therefore propose the following experiment: a beacon transmitter is connected to a linearly polarized NVIS antenna. The transmit frequency is chosen so that stable NVIS propagation is present during a major part of the day. A receive station located approximately $100 \mathrm{~km}$ away measures the signal strength of the RHCP and the LHCP components of the incoming wave continuously. The ratio between the LHCP and RHCP signal strength is calculated and plot 
against time. For this experiment a measurement system is realized using commercially-offthe-shelve equipment, completed with a few components specially designed for this experiment. An overview of the system components and their interconnections are shown as a block diagram in Figure 7.11 and described in the following paragraphs.

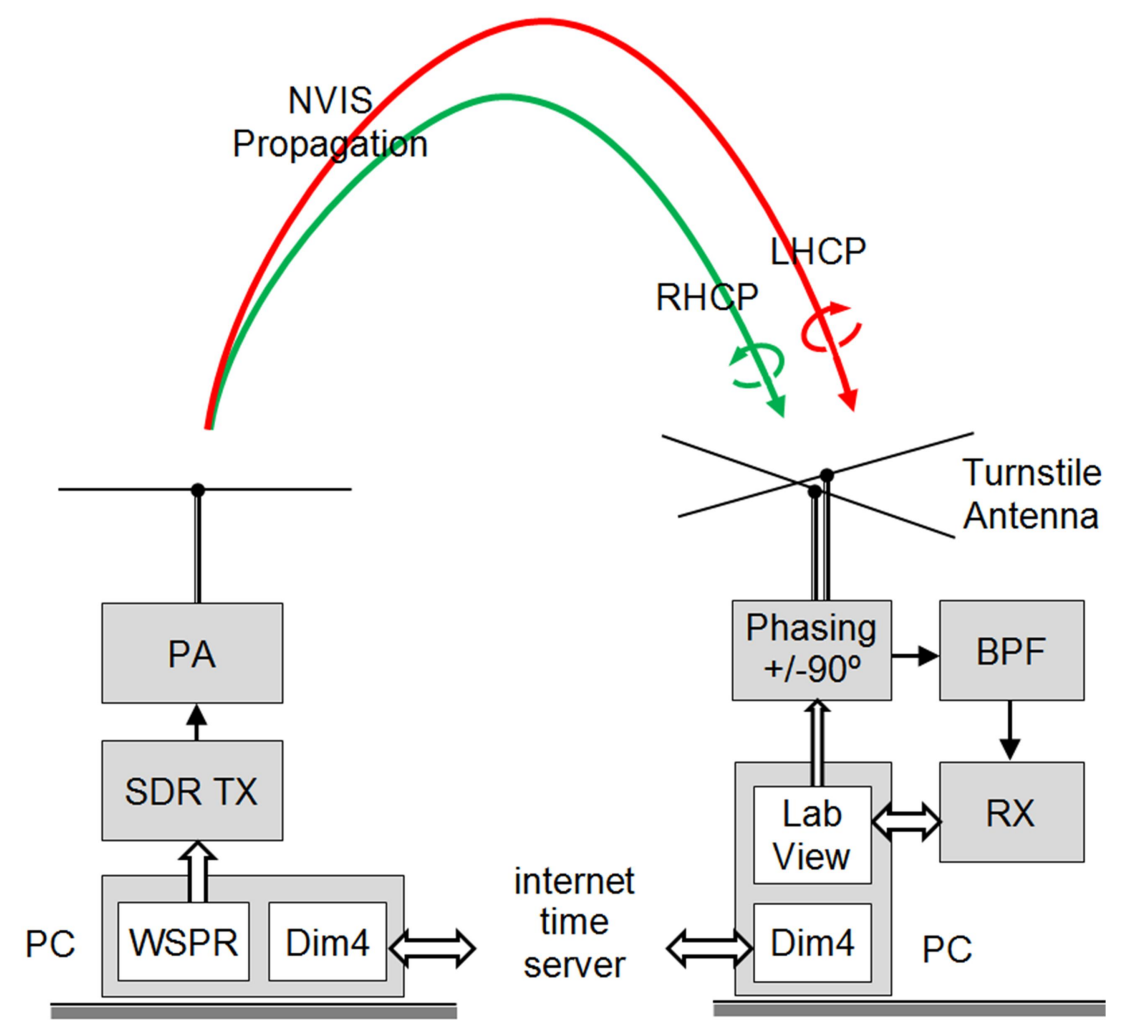

Figure 7.11 Block diagram of the experimental measurement system. A beacon transmitter (left) is connected to a linearly polarized NVIS antenna. A receive station (right) - located approximately $100 \mathrm{~km}$ away continuously measures the signal strength of the RHCP and the LHCP components of the incoming wave.

\subsubsection{Beacon transmitter}

A Software Defined Radio transmitter type Flex-Radio FLEX-6500 is used, followed by a Trans World Electronics T1000 linear amplifier with 300 Watts RF output power. Measured transmitter frequency stability is better than $0.1 \mathrm{~Hz} / 24$ hours. Measured output power stability is better than $0.1 \mathrm{~dB} / 24$ hours. The Weak Signal Propagation Reporter (WSPR) protocol [Taylor] is used to transmit the station ID and geographical coordinates in 1.5 Baud 4-FSK, with a necessary bandwidth of $6 \mathrm{~Hz}$. The WSPR protocol has a 2 minute periodicity, consisting of 110.6 second transmissions followed by a 9.4 second silence, synchronized to a standard time server accessed over the internet using Dimension4 software [Dimension4]. The high frequency and time accuracy allow for precise filtering at the receiver. A half-wave dipole antenna is used as a transmit antenna. It is suspended horizontally at a height of approximately 4 meters $(0.09 \lambda)$ above farmland soil. The antenna produces linear polarization with a broad main lobe towards the zenith. For high angles, the radiation 
pattern is omnidirectional. For low angles the antenna radiates lengthwise with vertical polarization. Therefore, to minimize ground wave coupling, it is oriented perpendicular to the direction of the receiver.

\subsubsection{Turnstile antenna}

A Turnstile Antenna [Kraus, 1988, pp. 726-729] was selected to measure the field strength of both characteristic waves. This antenna consists of two quadrature-fed perpendicular half wave dipole antennas and exhibits circular polarization for the steep elevation angles used in NVIS propagation. The polarization sense can be reversed by changing the phase difference of the dipoles from $+90^{\circ}$ to $-90^{\circ}$. On HF this Turnstile antenna can be realized with wire dipole elements suspended in an 'Inverted Vee' configuration from a single extendable mast, as shown in Figures 7.12 and 7.13. The co-polar and cross-polar antenna diagrams shown in Figures 7.14 and 7.15 are calculated using NEC-4.2 Method-of-Moments antenna simulation software [Burke, 2004]. The Sommerfeld ground model [Burke, 1981] is used to obtain realistic results near real ground. The model was created and analyzed with $4 \mathrm{Nec} 2$ [Voors, 2012]. Farmland soil was used in the calculations. Practical realization of the antenna can be seen in Figure 7.16.

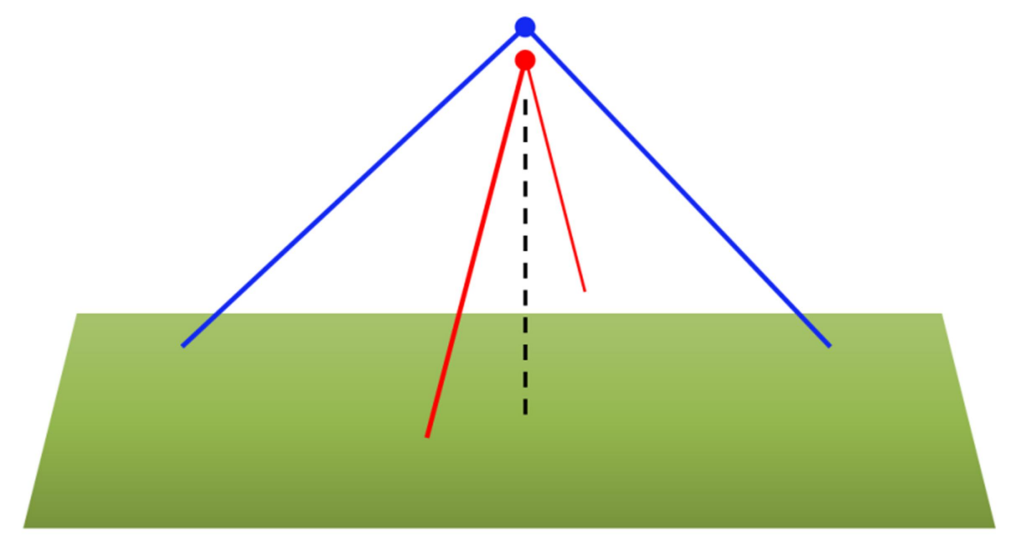

Figure 7.12 Turnstile antenna made of two quadrature-fed half-wave dipole antennas suspended in an 'Inverted Vee' configuration from a single extendable mast.

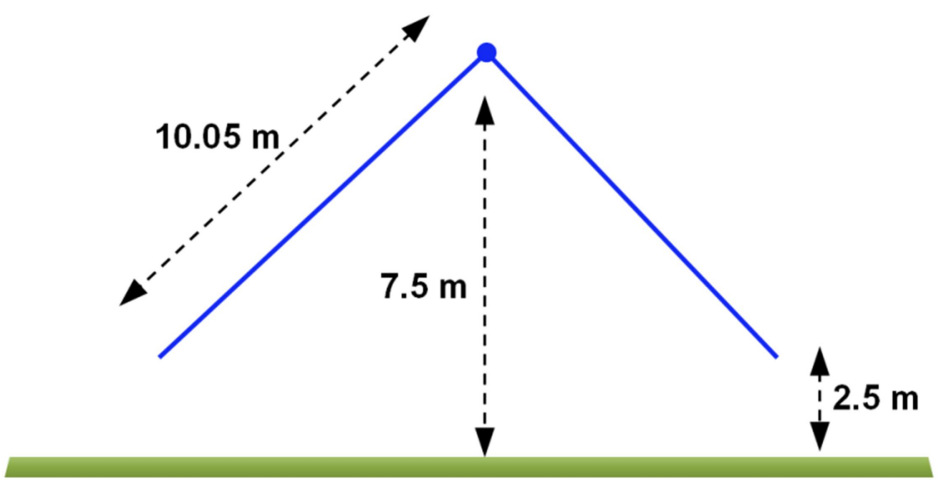

Figure 7.13 Dimensions of the dipole elements of the Turnstile antenna (wire radius $1 \mathrm{~mm}$ ). 


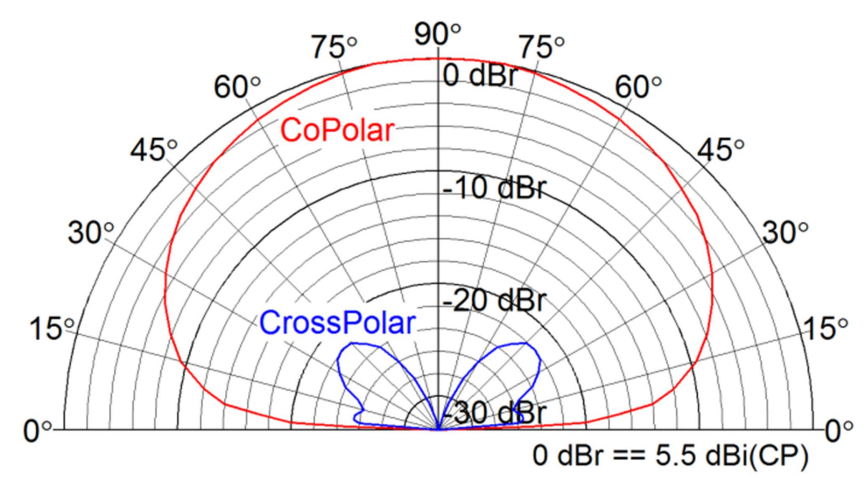

Figure 7.14 Vertical antenna diagram showing co-polar (red) and cross-polar (blue) circular polarization antenna gain of the Turnstile antenna, simulated using NEC-4.2 Method-of-Moments software. Farmland soil was used in the calculations.

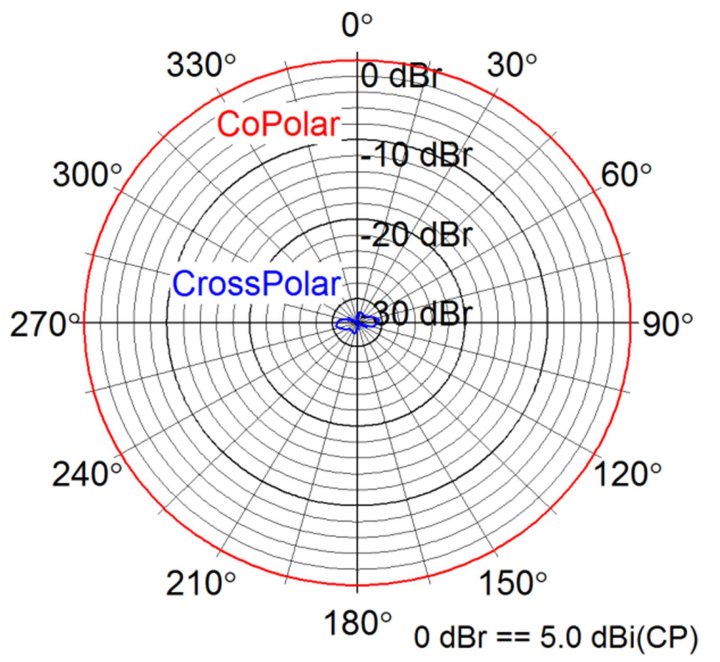

Figure 7.15 Horizontal antenna diagram at $70^{\circ}$ elevation showing co-polar (red) and cross-polar (blue) circular polarization antenna gain of the Turnstile antenna, simulated using NEC-4.2 Method-of-Moments software. Farmland soil was used in the calculations.

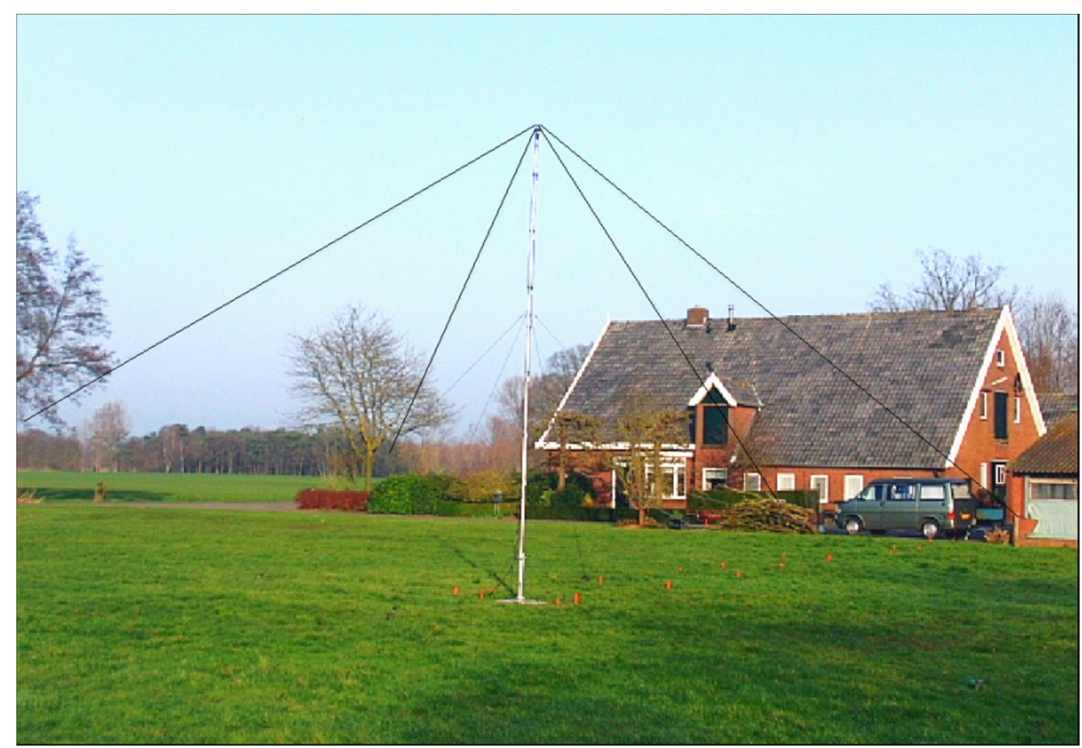

Figure 7.16 Turnstile antenna installed at the measurement location. The farmhouse and barn shown on the picture are at approximately 50 and $80 \mathrm{~m}$ distance. Other buildings are at $>800 \mathrm{~m}$ distance. 


\subsubsection{Balance transformers and feed lines}

Both dipole antennas are fed through 1:1 balance-unbalance transformers (baluns), type Diamond BU-50. As any difference in phase delay or attenuation of these baluns would degrade quadrature, 5 baluns are measured pairwise. Individual phase delays vary from $7.9^{\circ}$ to $7.0^{\circ}$. A matched pair is selected with an attenuation difference smaller than $0.05 \mathrm{~dB}$ and a phase difference smaller than $0.1^{\circ}$.

The baluns are connected through identical lengths (50 meters) of EcoFlex 10 doubly shielded coaxial cable. Both cables are taped on opposite sides of the antenna support (mast) and ferrite clamps are added every meter to suppress common-mode current that would otherwise influence the antenna radiation diagram. The horizontal part of the feed lines is buried approximately $70 \mathrm{~cm}$ below the ground, to avoid coupling with the antenna. The electrical length of both feed lines is measured; difference in phase delay is less $0.16^{\circ}$. From these measurements, the overall difference of cable and baluns is expected to be less than $0.1 \mathrm{~dB}$ and $0.5^{\circ}$.

\subsubsection{Phasing network}

The quadrature feed for the Turnstile antenna is realized using switched coaxial delay lines. The feed lines coming from the perpendicular dipoles are connected to a phasing box, in which one cable is lengthened with a quarter wave phasing line to provide $90^{\circ}$ phase shift. The other feed line is lengthened by a half wave phasing line that can be bypassed using coaxial relays. Depending on the position of these relays the phase difference between the dipoles antennas is now either $90^{\circ}$ or $-90^{\circ}$.

The phasing lines are connected to an RF combiner, type Merrimac PDNL-20-100. The RF combiner has a measured phase error $<0.03^{\circ}$ and an attenuation error $<0.04 \mathrm{~dB}$. The coaxial relays are Tohtsu CX-600M, with a measured insertion loss of $0.01 \mathrm{~dB}$ and isolation greater than $80 \mathrm{~dB}$. The phasing lines are made of Belden $\mathrm{H}-155$ doubly shielded coaxial cable. Their insertion loss is $0.25 \mathrm{~dB}$ and $0.5 \mathrm{~dB}$, respectively. To compensate for this, attenuators of 0.25 $\mathrm{dB}$ and $0.5 \mathrm{~dB}$ are inserted as indicated in the diagram. Two $6 \mathrm{~dB}$ attenuators are inserted between the antenna feeder line and the phasing unit to make the phase shift less dependent on the source impedance of the dipoles.

A block diagram of the phasing network is shown in Figure 7.17. The practical realization mounted in a transportation box is shown in Figure 7.18. The phase and amplitude difference of the completed phasing unit was measured using an Agilent E5062A Network Analyzer (NWA) and carefully aligned. Total quadrature error of the phasing unit is $<0.1^{\circ}$ and $<0.05 \mathrm{~dB}$. This makes the overall quadrature error in the Turnstile antenna $<0.6^{\circ}$ and $<0.15$ dB. 


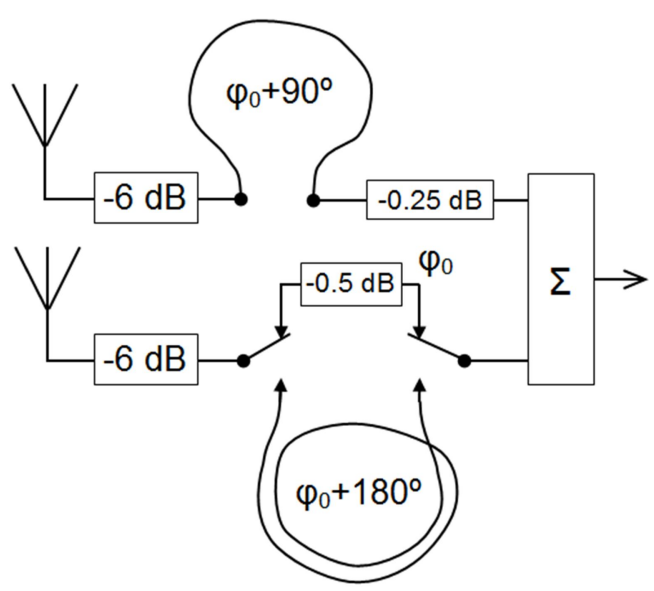

Figure 7.17 Block diagram of the phasing network for the Turnstile antenna using coaxial phasing lines to produce either- $90^{\circ}$ or $+90^{\circ}$ phase difference. The attenuation of the phasing lines is compensated with small attenuators $(0.25 \mathrm{~dB}$ and $0.5 \mathrm{~dB})$.

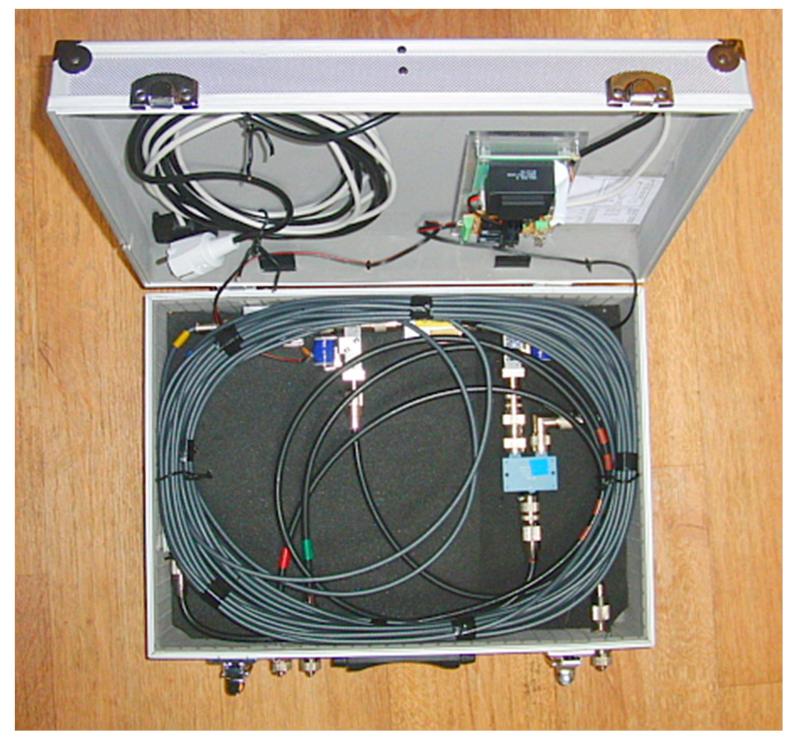

Figure 7.18 Practical realization of the phasing network for the Turnstile antenna.

\subsubsection{Antenna cross-polarization}

The maximum characteristic wave isolation that can be measured with our measurement system depends on the cross-polarization of the measurement antenna. To evaluate the influence of the quadrature error on the cross-polarization of the Turnstile antenna, the NEC-4.2 antenna model of paragraph 7.3.2 is used.

In the model the dipole elements are kept perfectly identical and perpendicular, and ground is perfectly flat, but quadrature errors are introduced to produce cross-polarization levels of $-20 \mathrm{~dB},-25 \mathrm{~dB}$ and $-30 \mathrm{~dB}$ at an elevation angle of $80^{\circ}$. These quadrature errors are plot in the graph of Figure 7.19 and ellipses are drawn through these points to delimit the areas in which a certain cross-polarization is achieved. 


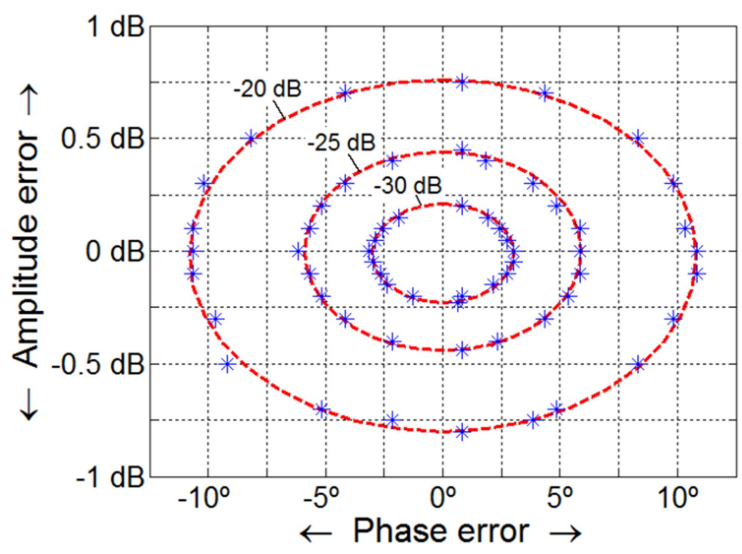

Figure 7.19 Simulated cross-polarization of the Turnstile antenna as a function of the amplitude and phase error of the phasing unit, assuming perfectly identical dipoles. Elevation angle is $80^{\circ}$.

A quadrature error of $0.6^{\circ}$ and $0.15 \mathrm{~dB}$ will result in a cross-polarization of approximately -32 $\mathrm{dB}$. However, the real cross-polarization value will depend also on the physical symmetry of the antenna wires and the homogeneity of the ground below them. For example, adding a random height error between 0 and 30 centimeters to the end height of the 4 dipole legs, while maintaining the leg length constant, will degrade the cross-polarization to values in between $-25 \mathrm{~dB}$ and $-29 \mathrm{~dB}$. Some specific combinations can also be found in which degradation is worse. This must be kept in mind when installing the measurement antenna system, and when interpreting measurement results.

\subsubsection{Measurement receiver}

The HF radio environment puts high demands on measurement receiver performance. A 24hour registration shows that the maximum total power at the antenna terminals of a dipole antenna is $-40 \mathrm{dBm}$, due to the accumulated power of high-power short-wave broadcast stations. At the same time, the minimum discernible signal power is $-135 \mathrm{dBm}$. The Intermodulation Free Dynamic Range of the receiver must therefore exceed $95 \mathrm{~dB}$. For our experiment a Rohde \& Schwarz FSMR26 measurement receiver was selected. This receiver provides a combined measurement uncertainty of $0.3 \mathrm{~dB}$ for $95 \%$ confidence on $7 \mathrm{MHz}$, with $100 \mathrm{~Hz}$ receiver bandwidth and RMS detector. The specified $-1 \mathrm{~dB}$ input compression point of the receiver is $+13 \mathrm{dBm}$, the $3 \mathrm{rd}$ order Input Intercept Point (IIP3) is $+17 \mathrm{dBm}$ and the $2 \mathrm{nd}$ order Input Intercept Point (IIP2) is $+35 \mathrm{dBm}$. We measured an IIP3 of $+18 \mathrm{dBm}$ at $7 \mathrm{MHz}$ for $200 \mathrm{kHz}$ spacing and a displayed average noise level (DANL) of $-135 \mathrm{dBm}$. The maximum allowed input power Pmax for which the 3rd order intermodulation products remain beneath the receiver noise floor can be calculated as:

$$
P_{\max }=I I P 3-(I I P 3-D A N L) / 3=18 \mathrm{dBm}-(18 \mathrm{dBm}+135 \mathrm{dBm}) / 3=-33 \mathrm{dBm}
$$

This leaves only $7 \mathrm{~dB}$ headroom, therefore a $7 \mathrm{MHz}$ band pass filter is added at the receiver input. This reduces the maximum total input power by $20 \mathrm{~dB}$ and increases Pmax to -13 
$\mathrm{dBm}$. Filter passband attenuation is $0.23 \mathrm{~dB}$ only. A $100 \mathrm{~Hz}$ receiver bandwidth is chosen for the measurements. This is sufficiently large to ensure fast settling of the detector, yet selective enough to reduce the probability of co-channel interference. Measurements using LHCP and RHCP are alternated every 5 seconds. Both data acquisition and polarization sense are controlled by a laptop computer using LabView software.

Time synchronization between transmitter and receiver is achieved by synchronizing both to the same standard time server, which is accessed over the internet with Dimension4 [Dimension4] software. The synchronization error is less than 0.05 second.

\subsection{MEASUREMENT RESULTS}

Using the system described in Section 7.3, dual circular polarization measurements were performed from Saturday 8 March 2014 14:51 UTC to Tuesday 11 March 2014 at 00:00 UTC. The beacon transmitter was located $53.18058^{\circ}$ North and $7.29503^{\circ}$ East. The measurement system was located $52.26153^{\circ}$ North, $7.62175^{\circ}$ East. Both locations are in rural areas in The Netherlands. The path length was $104.5 \mathrm{~km}$, azimuthal direction was $188^{\circ}$. As was shown in Chapter 4, this distance is sufficient for the NVIS signal to dominate the ground wave. At the time of the measurements the Smoothed Sunspot Number (SSN) was 67. Ionization was sufficiently high to use a frequency near $7 \mathrm{MHz}$ to obtain stable NVIS propagation for a large part of the day, and this frequency was also high enough to ensure E-layer transparency. The expected elevation angle, obtained by simulations, varies between $75^{\circ}$ and $80^{\circ}$ during daytime propagation and between $79^{\circ}$ and $86^{\circ}$ when the propagation path opens or closes. The ionosondes located at Dourbes $\left(50.1^{\circ} \mathrm{N}, 4.6^{\circ} \mathrm{E}\right)$, Juliusruh $\left(54.6^{\circ} \mathrm{N}, 13.4^{\circ} \mathrm{E}\right)$ and Chilton $\left(51.5^{\circ} \mathrm{N},-1.3^{\circ} \mathrm{E}\right)$ were monitored for sporadic E-layer (Es) patches that could disturb the measurements, but none were observed.

\subsubsection{Signal identification and signal-to-noise ratio}

During the 57 hour measurement interval a spectrogram (frequency-time graph or waterfall diagram) was recorded every 2.5 seconds, alternatingly on both antenna polarizations. In this spectrogram the beacon signal is easily identified, firstly by its transmit frequency and secondly by its precisely defined on-off pattern, as can be seen in Figure 7.20. The 'off' periods are used to verify the absence of on-channel interference. Fortunately, no data had to be discarded because of interference, the beacon signal was sufficiently strong and the frequency clear. The 'off' periods in the beacon signal were also used to estimate the instantaneous signal-to-noise ratio (SNR), which was greater than $65 \mathrm{~dB}$ during daylight hours. At night, with no apparent NVIS propagation, the SNR was still greater than $20 \mathrm{~dB}$. The latter can be seen on 9 March 2014 at 02:30h UTC (Figure 7.22), when the beacon transmitter has a short failure due to human error. 


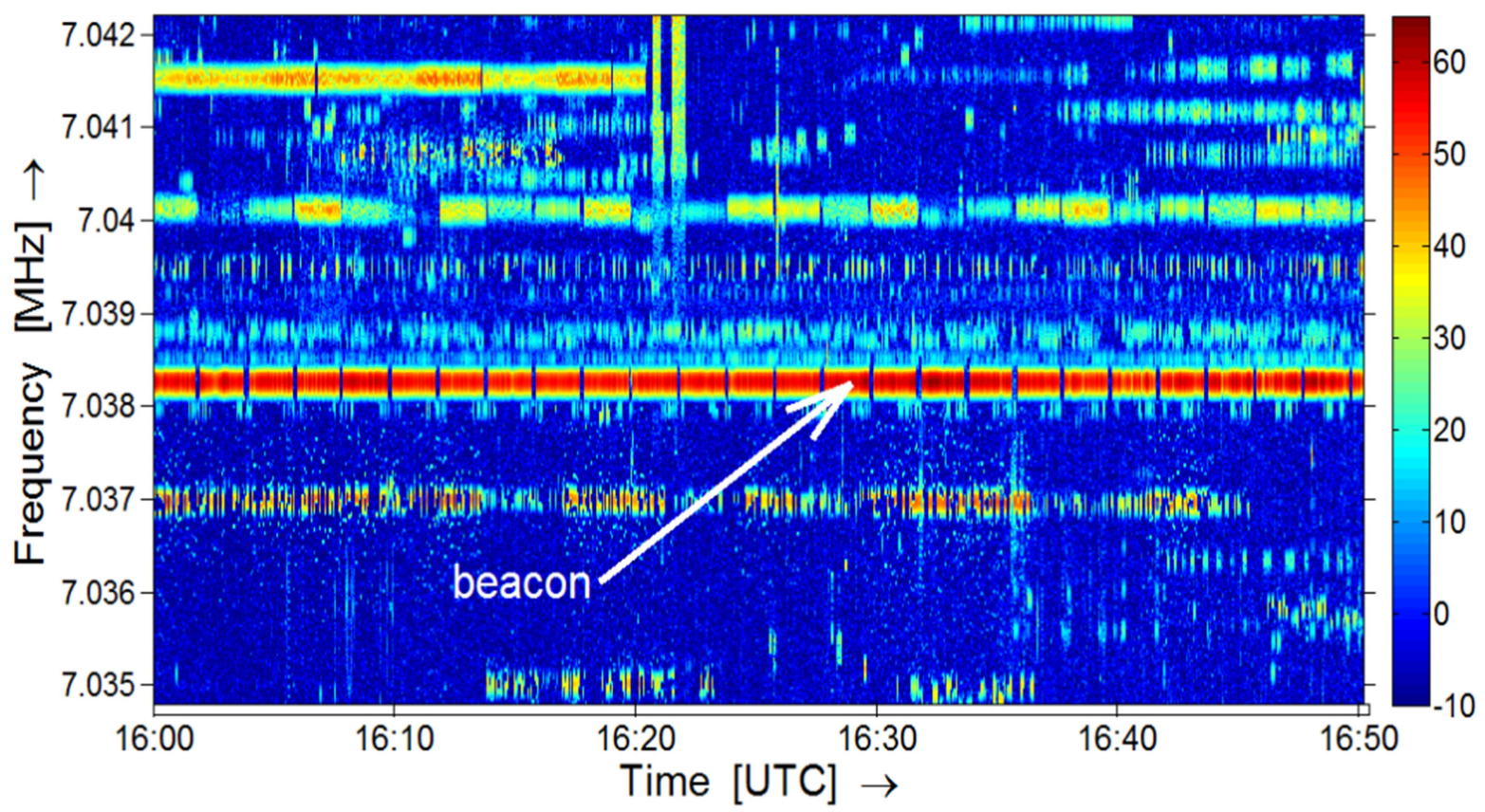

Figure 7.20 Spectrogram showing the strong and cyclic signal of the beacon transmitter and other radio signals on adjacent frequencies. Color scale shows received signal strength in $\mathrm{dB} \mu \mathrm{V}$. Measurement made on 9 March 2014.

\subsubsection{Dual circular polarization measurements}

The beacon frequency was filtered from the spectrogram data and the remaining data was time gated to retain only the samples in which the beacon transmitter was switched on. The signal strength of the beacon for both LHCP and RHCP reception is plot in Figures 7.21, 7.22 and 7.23. For ease of interpretation, the 57-hour continuous measurement is presented in 24-hour intervals. Signal strength of the extraordinary wave (RHCP) is shown in green, of the ordinary wave (LHCP) in red. The lighter colored pixels are the individual measurement samples recorded every 5 seconds; the solid lines show a 2.5-minute floating average. Daylight on 9 March 2014 was from 06:02 to $16: 28$ UTC at path midpoint $\left(52.72^{\circ} \mathrm{N}, 7.46^{\circ} \mathrm{E}\right)$. NVIS propagation started every morning around sunrise at approximately 06:10 UTC and ended between 20:00 and 22:00 UTC, several hours after sunset, with a large day-to-day variation. A blue trace is added below the measured signal strength curves, showing the ratio of the signal strength of the ordinary and extraordinary waves. The morning and evening Happy Hour can be clearly seen. The morning Happy Hour is shorter than the evening Happy Hour, as was predicted in paragraph 7.2.1. 


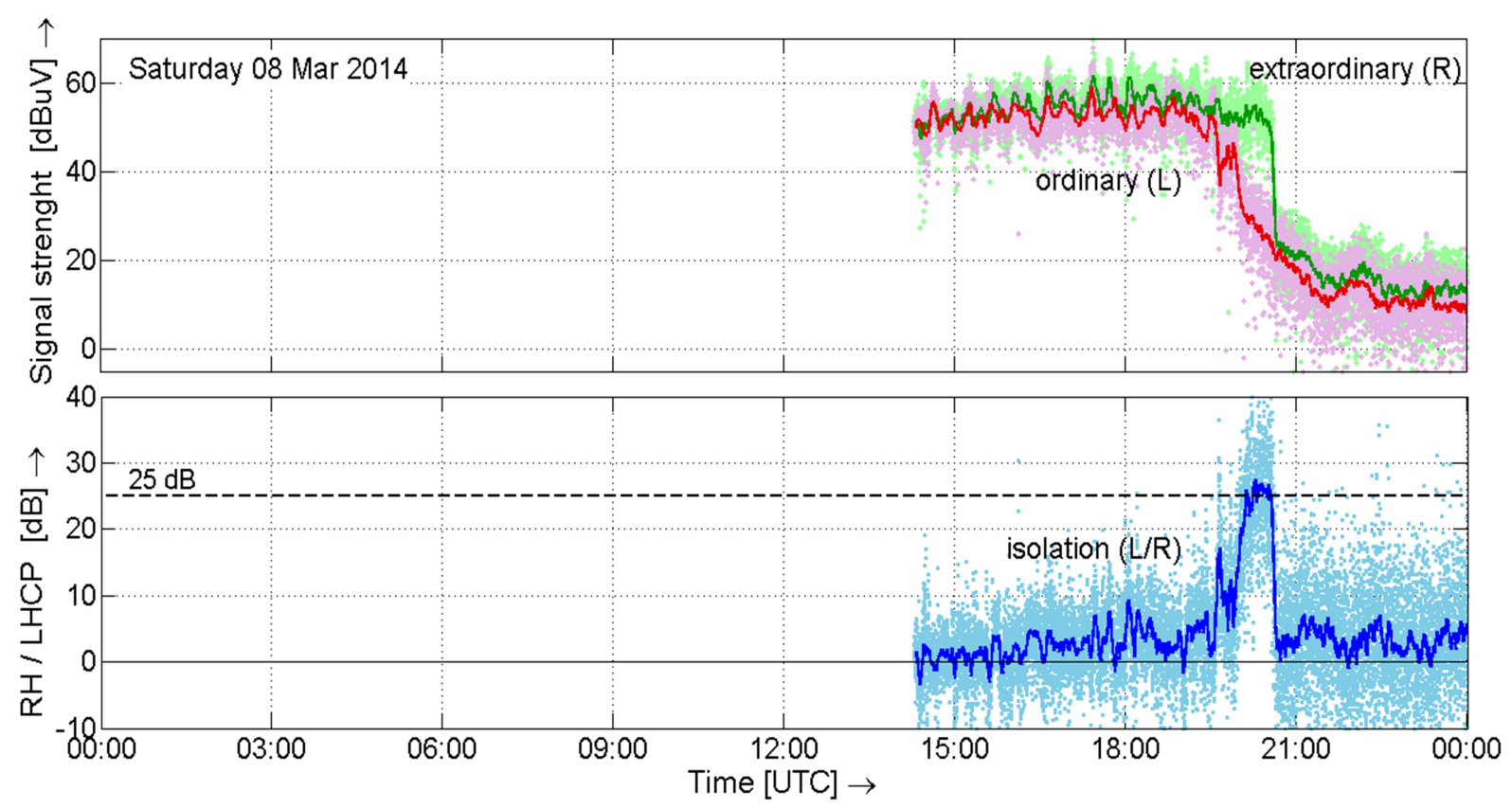

Figure 7.21 Start of the measurements on Saturday 8 March 2014 - 14:51 UTC. Signal strength of the extraordinary wave is shown in green, of the ordinary wave in red. The ratio of the two is shown in blue. Daylight ends at 16:28 UTC. NVIS propagation ends around 20:35 UTC. The evening Happy Hour shows approximately $25 \mathrm{~dB}$ characteristic wave isolation.

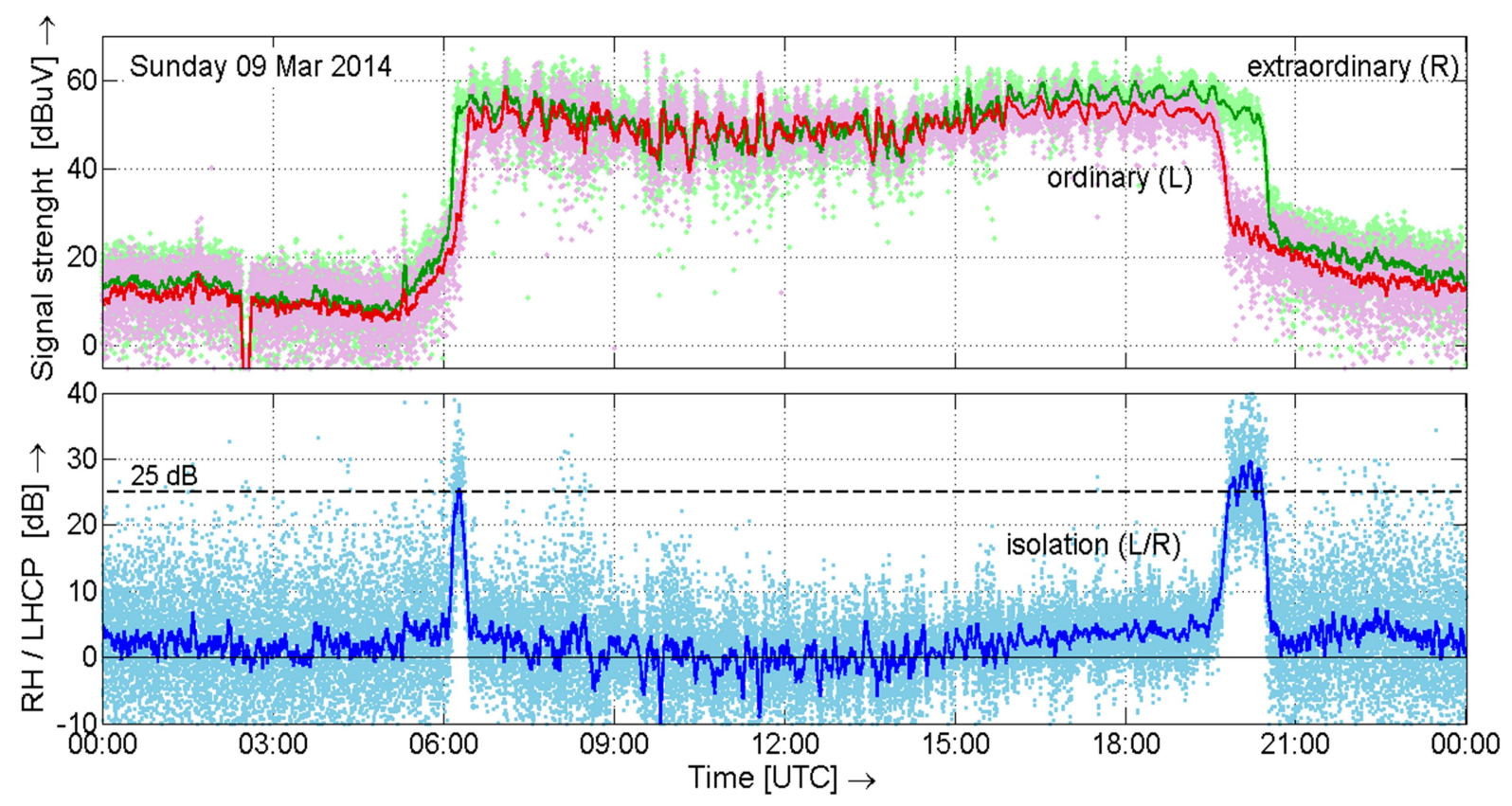

Figure 7.22 Continuation of the measurements on Sunday 9 March 2014. Daylight is from 06:02 to 16:28 UTC. NVIS propagation starts around 06:03 UTC and ends around 20:25 UTC. Both 'Happy Hours' show approximately $25 \mathrm{~dB}$ characteristic wave isolation. The short signal loss at 02:30 UTC due to beacon transmitter failure shows that SNR $>20 \mathrm{~dB}$ at night. 


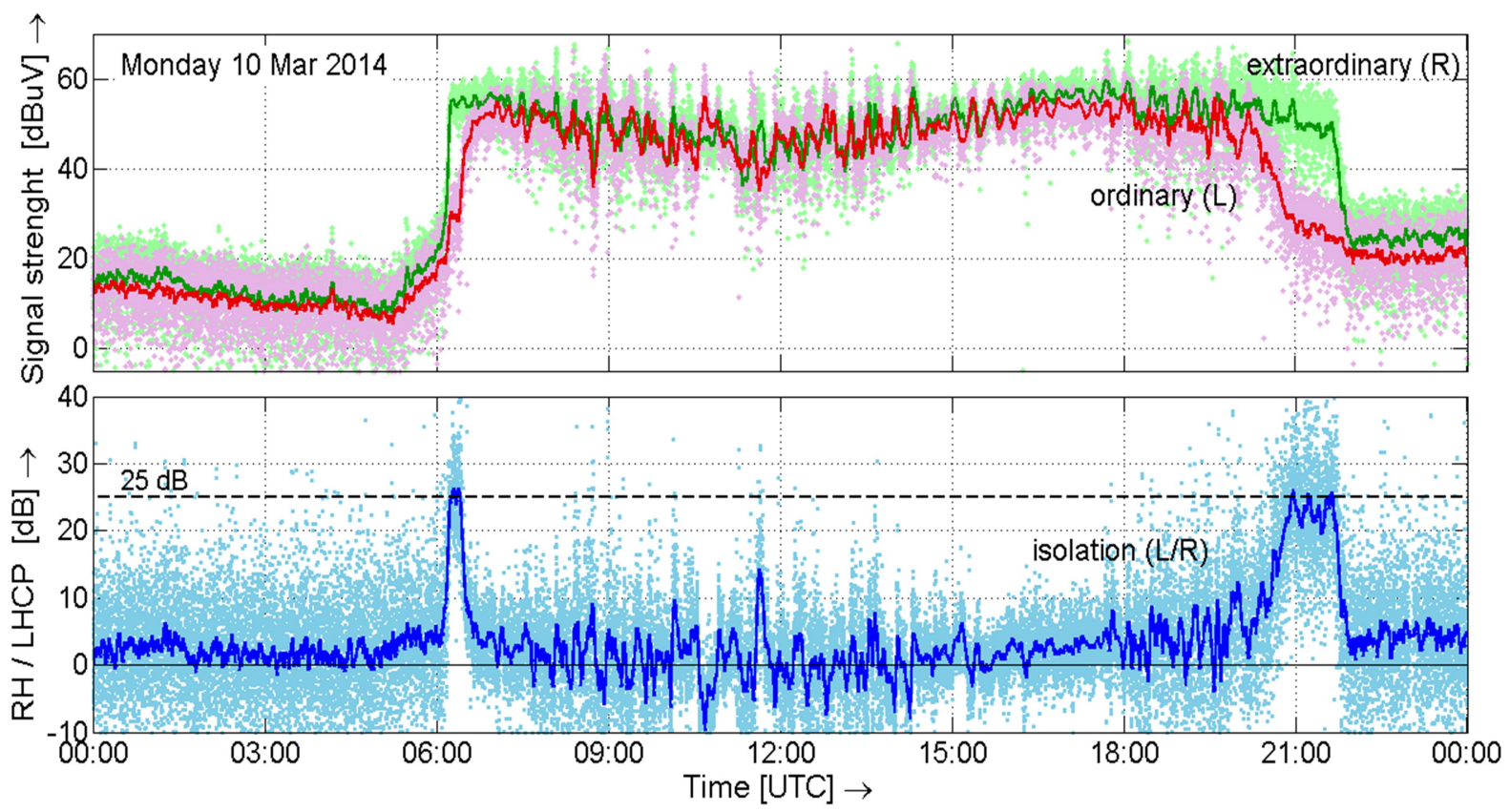

Figure 7.23 Continuation of the measurements on Monday 10 March 2014. Daylight is from 06:02 to 16:28 UTC. NVIS propagation starts around 06:10 UTC and ends around 21:40 UTC. Both 'Happy Hours' show approximately $25 \mathrm{~dB}$ characteristic wave isolation.

\subsection{ANALYSIS AND DISCUSSION}

Figures 7.21-7.23 prove the existence of a morning and evening Happy Hour interval, in which only RHCP waves are received, consistent with preliminary measurements in 2009, described in Chapter 6. As the previous measurements were made near the sunspot cycle minimum and these extended measurements were made near the sunspot cycle maximum, independence of the position in the sunspot cycle is demonstrated. In all 5 Happy Hour intervals instantaneous RHCP/LHCP ratios of up to $35 \mathrm{~dB}$ are observed, as well as a 2.5minute average of approximately $25 \mathrm{~dB}$.

\subsubsection{Morning Happy Hour observations}

The measurements during the morning Happy Hour of 9 and 10 March 2014 are shown in Figures 7.24 and 7.25. Again, the lighter colored pixels represent the individual measurement samples recorded every 5 seconds; the solid lines show a 2.5-minute floating average. The morning intervals consistently started a few minutes after sunrise with a sudden increase of the received signal strength of the extraordinary wave. The signal strength increased by $35 \mathrm{~dB}$ in a 10 minute interval, just as if the propagation channel 'switches on'. After that moment, the received polarization is nearly perfectly RHCP. The rise of the signal strength caused by the ordinary wave started later and was more gradual than that of the extraordinary wave. In the interval where the extraordinary wave propagated and the ordinary wave did not, a characteristic wave isolation of $25 \mathrm{~dB}$ was measured. 

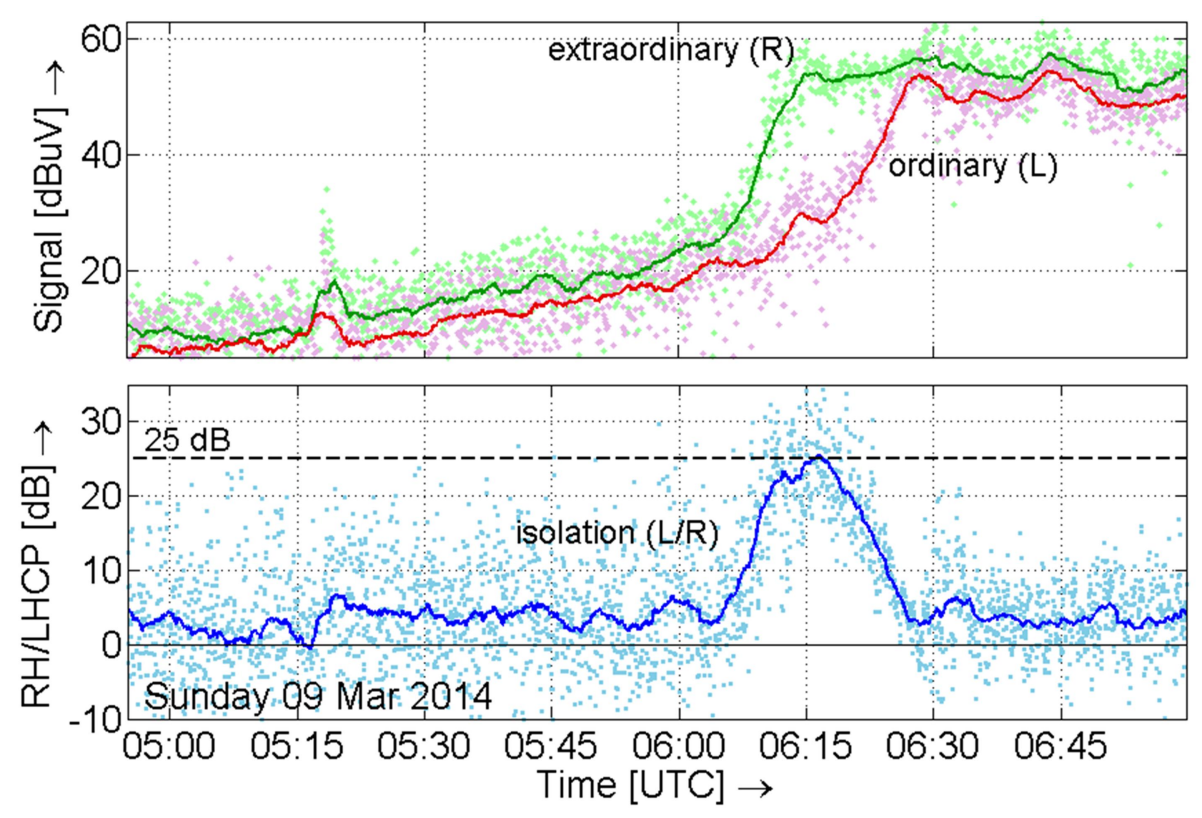

Figure 7.24 Signal strength of the ordinary (red) and extraordinary wave (green) and their ratio (blue), measured during the morning Happy Hour of Sunday 9 March 2014. The peak in the blue trace shows the characteristic wave isolation during Happy Hour.
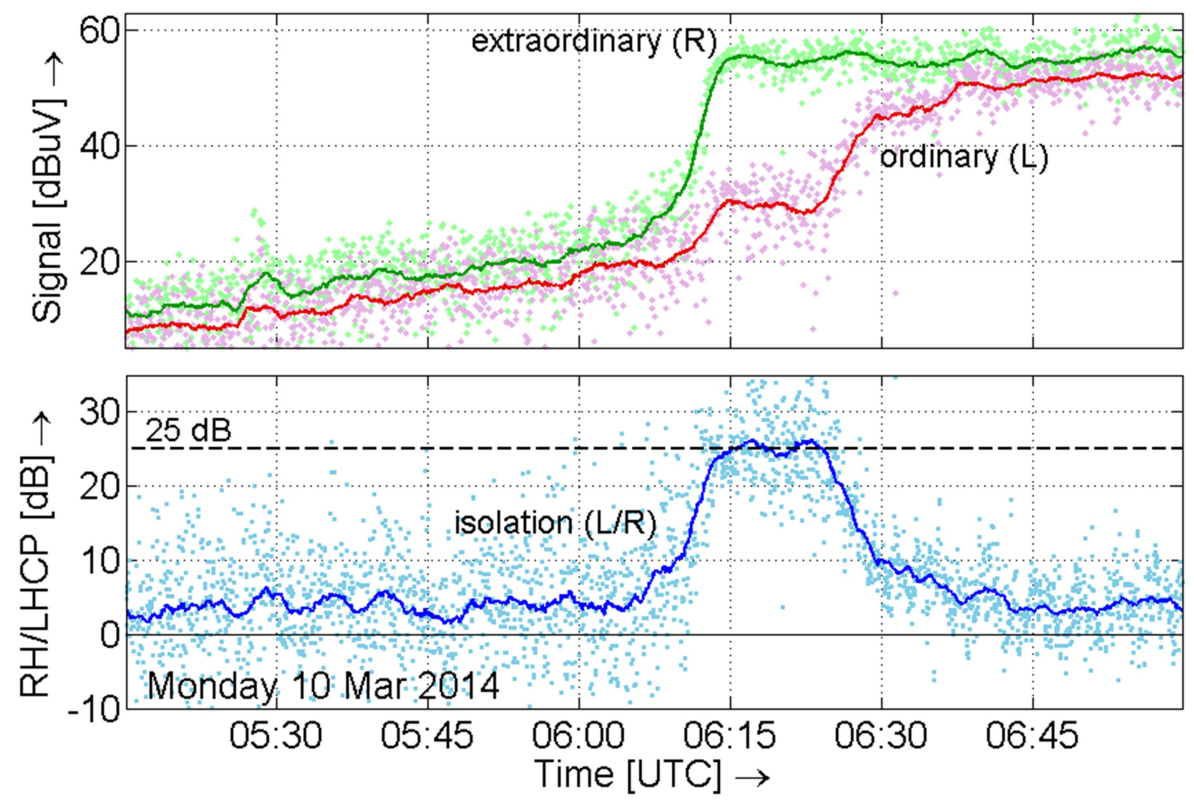

Figure 7.25 Signal strength of the ordinary (red) and extraordinary wave (green) and their ratio (blue), measured during the morning Happy Hour of Monday 10 March 2014.

The onset of the propagation before the Happy Hour interval was gradual; a slight increase of the signal strength of the extraordinary wave began 1 to 4 hours before the Happy Hour interval started. Therefore the baseline value for these ratio values was calculated over a longer time interval. 


\subsubsection{Evening Happy Hour observations}

The measurements during the evening Happy Hour intervals of 8, 9 and 10 March 2014, which occurred several hours after sunset, are shown in Figures 7.26 to 7.28. The NVIS propagation of the ordinary wave started to decay 1 hour ( 8 March) to 2 hours (10 March) earlier than the NVIS propagation of the extraordinary wave. During the Happy Hour interval RHCP waves were received at the measurement site.
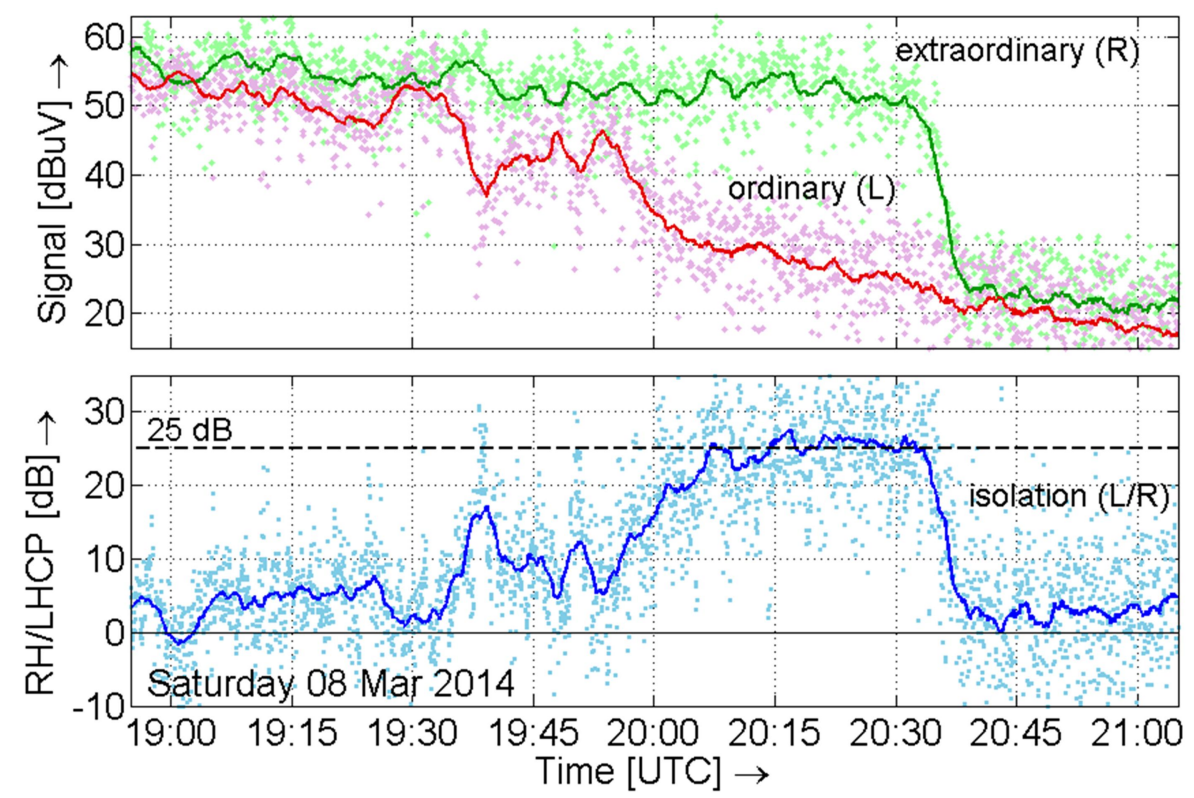

Figure 7.26 Signal strength of the ordinary (red) and extraordinary wave (green) and their ratio (blue), measured during the evening Happy Hour of Saturday 8 March 2014.
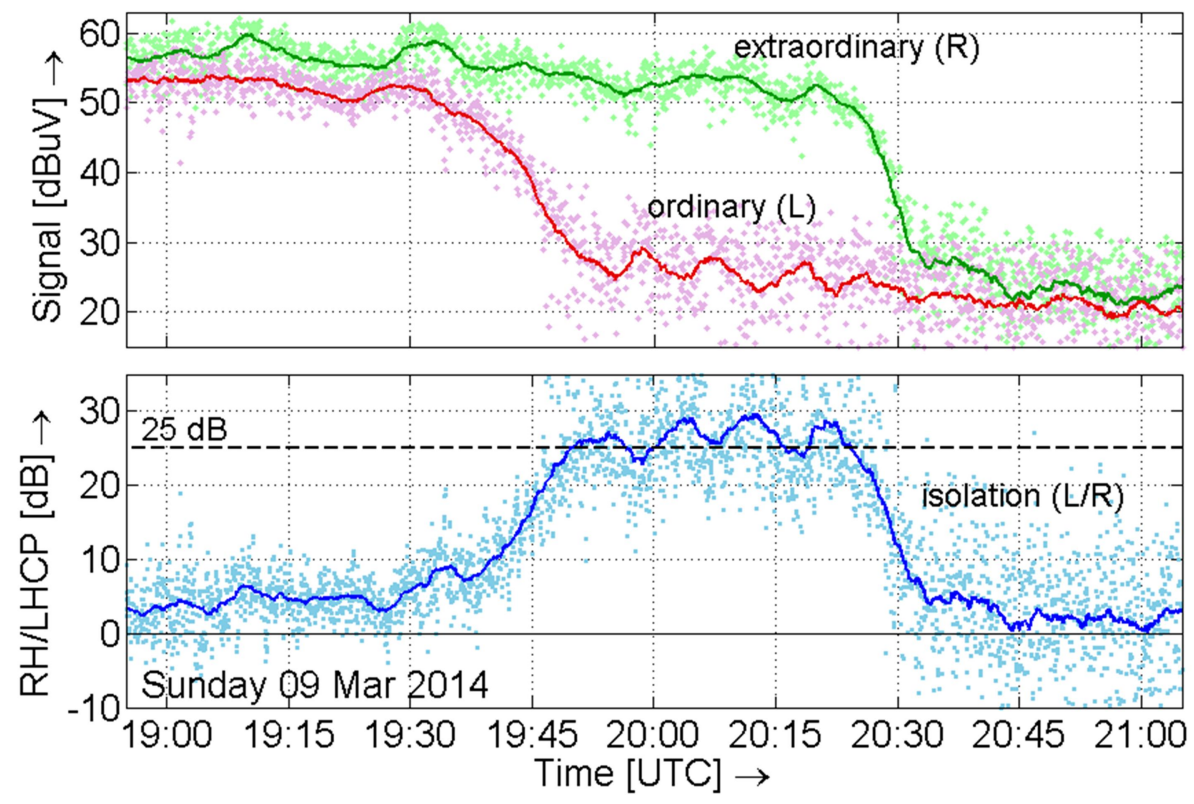

Figure 7.27 Signal strength of the ordinary (red) and extraordinary wave (green) and their ratio (blue), measured during the evening Happy Hour of Sunday 9 March 2014. 

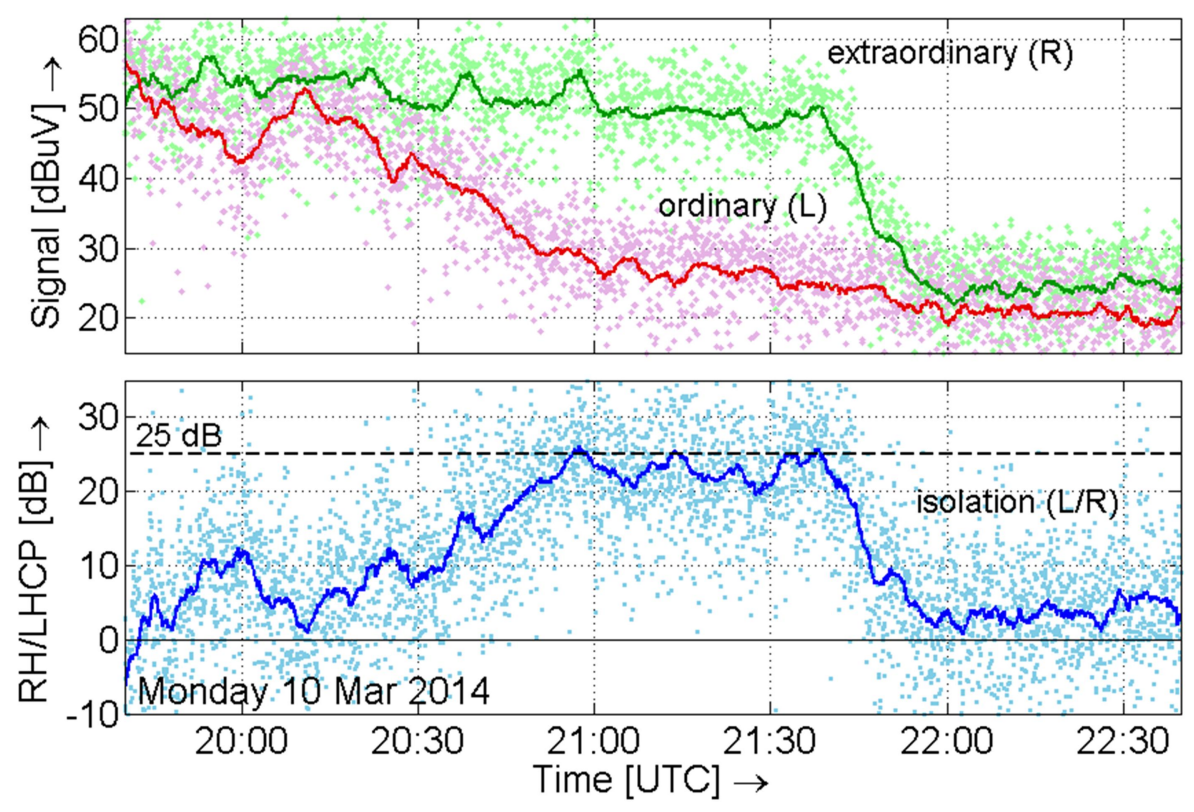

Figure 7.28 Signal strength of the ordinary (red) and extraordinary wave (green) and their ratio (blue), measured during the evening Happy Hour of Monday 10 March 2014.

The extraordinary wave exhibits a stable signal level up to the end of the NVIS propagation period, after which the signal strength dropped quite abruptly. The evening Happy Hour had a longer duration than the morning Happy Hour and its onset and duration showed a larger day-by-day variation. Measured characteristic wave isolation was $25 \mathrm{~dB}$.

\subsubsection{Interpretation of measured characteristic wave isolation}

The measured characteristic wave isolation values were consistently around $25 \mathrm{~dB}$. This is 12 $\mathrm{dB}$ greater than the isolation measured during earlier experiments (Chapter 6), to be attributed to the significantly improved quadrature feeding network and antenna symmetry. The true characteristic wave isolation may be higher still. In Figure 7.29, showing the morning Happy Hour of 10 March 2014, we see the steep rise of the extraordinary wave signal (green), and the smoother ascend of the ordinary wave signal (red). The expected slope of the red trace is added as a dashed black line. We remark an abrupt step in the red trace when the green trace rises. This indicates a leakage from the RHCP channel to the LHCP channel in the measurement system, rather than an ionospheric phenomenon. 


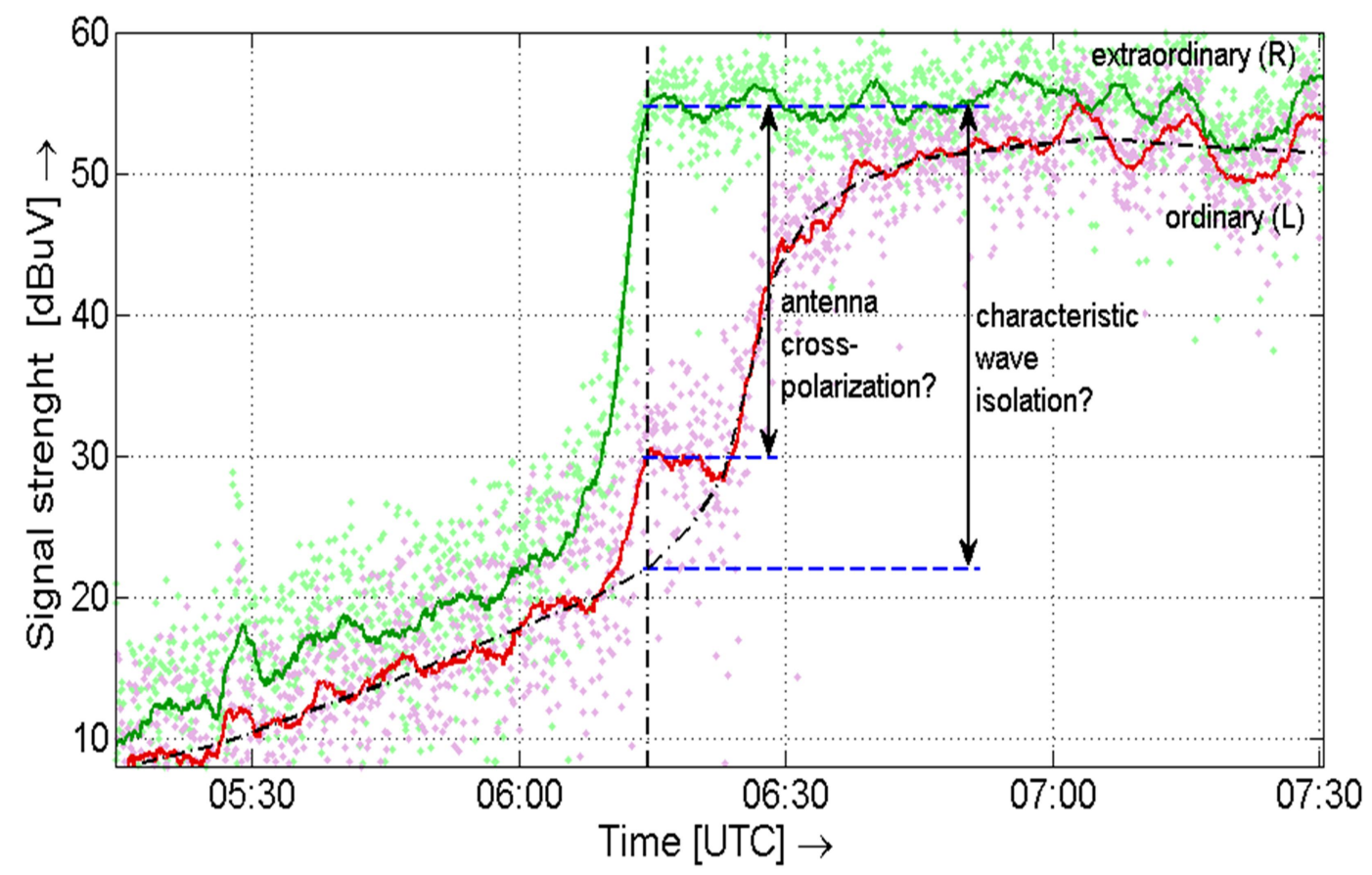

Figure 7.29 The measurement during the morning Happy Hour of 10 March 2014 shows an abrupt step in the ordinary wave signal, possibly indicating insufficient antenna cross-polarization.

There are three interpretations possible:

(a) The cross-polarization of the measurement antenna is not limiting the measurement and the characteristic wave isolation is exactly $25 \mathrm{~dB}$.

(b) The cross-polarization of the measurement antenna limits the measurement range to $25 \mathrm{~dB}$ and the characteristic wave isolation is greater than $25 \mathrm{~dB}$.

(c) The characteristic waves are slightly elliptically polarized, with characteristic wave isolation greater than $25 \mathrm{~dB}$. The cross-polarization of the measurement antenna is greater than $25 \mathrm{~dB}$ for perfectly circular polarization, but its cross-polarization is lower for the incoming waves that are elliptical.

The measurements performed here are not conclusive on this issue. Exclusion of option (a) is only possible by in-situ measurement of the cross-polarization of the measurement antenna. However, due the abrupt step observed in the ordinary wave signal, authors favor interpretation (b) or (c). To differentiate between these two, absolute polarization measurements are necessary. This could be achieved by simultaneously measuring both amplitudes and the phase difference on both ports of the Turnstile antenna using a synchronous dual channel measurement receiver. If the incoming waves are indeed slightly elliptical, orthogonality can be restored by adapting phase and amplitude in the receiver [Chu, 1971]. 


\subsubsection{Nighttime propagation observations}

After the NVIS propagation path closed a clearly discernable beacon signal remained, which had the typical flutter fading normally associated with ionospheric reflection or scattering. The character of this signal was unlike ground wave, which is more stable. Although the beacon signal was $45 \mathrm{~dB}$ lower than during daytime, the SNR was still more than $20 \mathrm{~dB}$. This can be seen in Figure 7.22 at at 02:30h: the beacon transmitter had a short failure and the recorded signal strength decreased approximately $20 \mathrm{~dB}$. Previous researchers assumed that the residual propagation at night is either due to scattering on irregular patches of higher ionization in the ionosphere [Wheeler, 1966] or due to side scatter on the ground at a large distance [Gibson, 1991]. No means where available during this experiment to measure azimuth and elevation angle or absolute polarization, therefore no further analysis could be made of this phenomenon. If the night-time propagation is due to a scattering mechanism, dual circular polarization diversity will probably not be effective at night, as the polarization will probably be lost in the process.

\subsection{CONCLUSIONS}

The measured isolation between the ordinary and extraordinary wave in Near Vertical Incidence Skywave (NVIS) propagation exceeds $25 \mathrm{~dB}$. The measurements were performed using a dual circularly polarized measurement antenna. Observations suggest that still higher isolation can be achieved by further adapting the antenna to the polarization of the incoming waves that may be slightly elliptical. Two highly isolated paths can be created on the same frequency using dual circularly polarized antennas on both transmit and receive sides of the link, effectively doubling the data transfer capacity of the link. Alternatively, without modification on the transmit side, a dual circular receive antenna can be used to implement an effective receive diversity system to fight fading in NVIS links, as will be shown in Chapter 8. The measurements were done at mid-latitudes in the Northern hemisphere $\left(53^{\circ} \mathrm{N}, 6^{\circ} \mathrm{E}\right)$, and results may depend on the latitude chosen. The measurements made use of the Happy Hour phenomenon: when the NVIS propagation path just opens up, or nearly closes, only the extraordinary wave propagates and the ordinary wave passes through the ionosphere or is absorbed. 
Interaction of Antenna and Propagation Mechanism 


\title{
8
}

\section{Characteristic Wave Diversity in Near Vertical Incidence Skywave propagation}

\begin{abstract}
In Near Vertical Incidence Skywave (NVIS) propagation, effective diversity reception can be realized using a dual channel receiver and a dual polarization antenna with polarization matched to the (left hand and right hand) circular polarization of the characteristic waves propagating in the ionosphere. This chapter presents measurements on NVIS-signals received via ionospheric F-layer reflection using a dual circular polarization receive antenna, demonstrating a reduction of the fading margin by 8-11 $d B$ over a single circular polarization antenna. The measurements were performed at a mid-latitude location in the Northern hemisphere, over a distance of $110 \mathrm{~km}$.
\end{abstract}

Published as: B. A. Witvliet, E. van Maanen, G. J. Petersen, A. J. Westenberg, M. J. Bentum, C. H. Slump, R. Schiphorst, "Characteristic Wave Diversity in Near Vertical Incidence Skywave Propagation," presented at European Conference on Antennas and Propagation, Lisbon, Portugal, April 2015. 
Interaction of Antenna and Propagation Mechanism 


\subsection{INTRODUCTION}

In areas where no telecommunication infrastructure exists [Hervás, 2013] or when the existing infrastructure is destroyed by disaster [Kwasinski, 2006; Kobayashi, 2014; Comfort, 2006 b], ionospheric radio wave propagation may be used to provide telecommunication over large distances without an intermediate human-made infrastructure. The ionosphere, an ionized layer around the earth that starts at a height of approximately $80 \mathrm{~km}$ altitude and has a maximum electron density at approximately $300 \mathrm{~km}$, refracts radio waves towards earth, enabling radio communication over distances up to thousands of kilometers. The ionosphere is frequency selective and the reflection height depends on the frequency of the emitted radio wave, its angle of incidence and the ionization profile of the ionosphere. During daytime, vertically emitted waves with a frequency below $4 \mathrm{MHz}$ are reflected at $80 \mathrm{~km}$ height, while frequencies between $4 \mathrm{MHz}$ and approximately $10 \mathrm{MHz}$ are reflected at much higher altitudes, ranging from 250 to $350 \mathrm{~km}$. Therefore, the ionosphere is often depicted as a set of reflecting layers, with the E-layer positioned the height of $80 \mathrm{~km}$ and the F-layer at a height varying from 250 to $350 \mathrm{~km}$. While such layers do not exist physically, the associated denominations are commonly used to describe ionospheric propagation modi. Ionospheric radio propagation can be used to realize continuous coverage starting directly at the transmitter and up to $200 \mathrm{~km}$ radius (or more) when a transmit frequency below the equivalent vertical frequency of the ionosphere [Davies, 1990, pp. 157-158] is used. Typical operating frequencies range from 3 to $10 \mathrm{MHz}$, depending on location and ionospheric parameters.

Due to the great reflection altitude, even the coverage of an area with $200 \mathrm{~km}$ radius requires nearly vertically launched radio waves. As was demonstrated with simulations and measurements in Chapter 4, elevation angles between $70^{\circ}$ and $90^{\circ}$ are needed to cover an area of $400 \times 400 \mathrm{~km}$ using F-layer reflection. This propagation mechanism, depicted in Figure 8.1, is known as 'Near Vertical Incidence Skywave' (NVIS). Ionospheric radio wave propagation is prone to 'fading': large and seemingly random short-term variations in received signal strength [Davies, 1990, pp. 232-239]. This fading is caused by slow variations in the ionospheric electron density profile, scattering against irregularities in the ionosphere and propagation via multiple paths through the ionosphere, which causes interference between the incoming waves arriving at the receiver. Especially the deep fades caused by multipath propagation require a large link margin to sustain communication during the signal minima. In this chapter we define this 'fading margin' as the additional margin that has to be reserved in the link budget to compensate for the minima in the fading channel, compared to a channel without fading.

In the ionosphere itself the electromagnetic energy of the incoming radio waves propagates in the form of two orthogonal characteristic waves, as was shown in Chapter 6. 


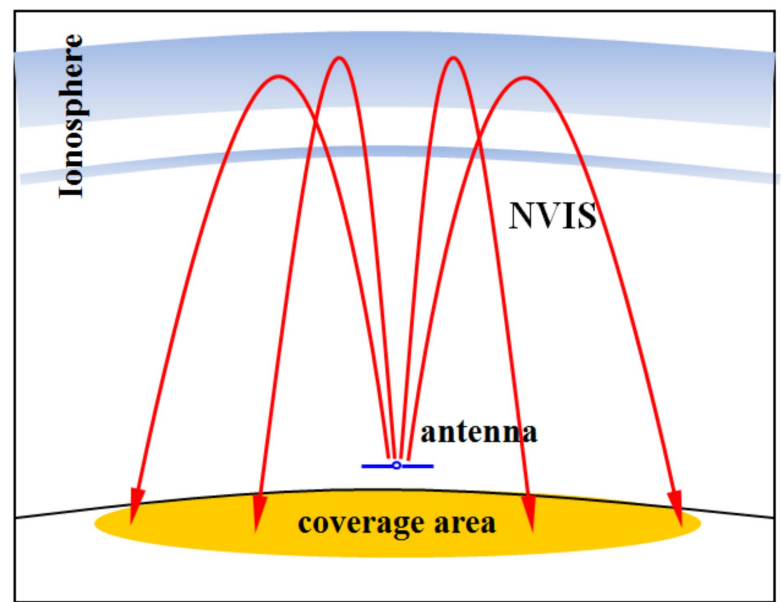

Figure 8.1 Near Vertical Incidence Skywave (NVIS) propagation: electromagnetic waves radiated nearly vertically are reflected by the ionosphere and cover a continuous area around to the transmitter.

As each of these characteristic waves travels an independent path and suffers different attenuation, their recombination at the receive antenna causes strong multipath and polarization fading [Davies, 1990, p. 235]. If a circularly polarized receive antenna is used, only one of the characteristic waves is received and the fading caused by interference of the characteristic waves is reduced. Further fading reduction can be achieved by creating a diversity system using a dual circular polarization receive antenna, employing the separate reception of the characteristic waves. In this chapter, measurements are presented showing that the required fading margin in NVIS propagation can be reduced 8-11 dB by using this Characteristic Wave Diversity method.

The chapter is structured as follows: Section 8.2 discusses the role of which characteristic waves in the ionosphere play in polarization fading. Section 8.3 describes an experiment demonstrating the effectiveness of Characteristic Wave Diversity. The results of this experiment are analyzed in Section 8.4. The chapter concludes with a discussion on the impact this concept may have on NVIS radio systems and a brief description of planned further experiments.

\subsection{CIRCULARLY POLARIZED CHARACTERISTIC WAVES IN NEAR VERTICAL INCIDENCE SKYWAVE}

Linearly polarized radio waves that enter the ionosphere are split up in two circularly polarized characteristic waves. This was already shown by Appleton [1932] during his research on the relationship between over-the-horizon propagation of short radio waves (<200 meters) and the Kennely-Heavyside layer [Kennely, 1902] between 1929 and 1932. He observed double ionospheric reflections on a transmitted RF pulse, the first reflection to arrive with right-hand circular polarization (RHCP), the second with left-hand circular polarization (LHCP). Figure 8.2 shows a recording of one of their experiments over $5 \mathrm{~km}$ 
distance. Appleton's magneto-ionic theory [Appleton, 1933] explaining this phenomenon is discussed in detail by Ratcliffe [1962]. Ratcliffe shows that only these circularly polarized waves can propagate in the ionosphere and names them 'characteristic waves'. Simulations presented in Section 7.2 show that both characteristic waves travel over independent paths through the ionosphere, often with large physical separation. Measurements presented in Section 7.4 demonstrate that, once excited, the isolation between both characteristic waves remains large (more than $25 \mathrm{~dB}$ ). As both characteristic waves travel different paths through the ionosphere, each one suffers different attenuation and delay. When leaving the ionosphere, the downward wave is a summation of both characteristic waves and has arbitrary polarization, which may rapidly vary between elliptical and linear polarization with random orientation. This will cause additional fading on linearly polarized antennas due to varying polarization mismatch: 'polarization fading'.

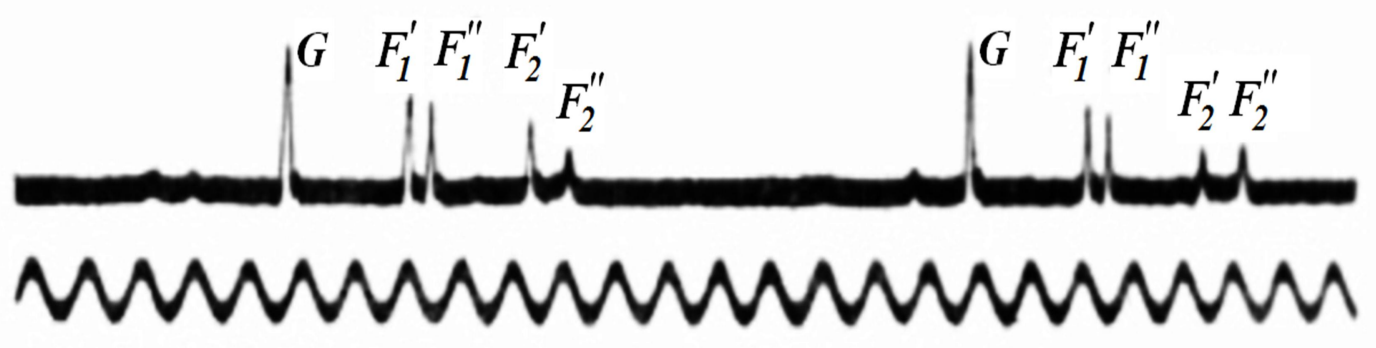

Figure 8.2 Pulse delay measurements by Appleton and Builder in 1932 over 5 km distance. Upper trace: pulses are received first via ground wave $(\mathrm{G})$, then twice via the F1-layer (F1' and F1") and twice via the F2-layer (F2' and F2"). Lower trace: a $1115 \mathrm{~Hz}$ sine wave serving as time reference.

\subsection{CHARACTERISTIC WAVE DIVERSITY EXPERIMENT}

The additional fading caused by polarization fading can be reduced by using a circularly polarized receive antenna, as such an antenna will only receive one of the characteristic waves, and characteristic wave interference will not occur. However, it is much more effective to use a dual-polarization receive antenna, and separately use the power transferred by both characteristic waves in a diversity system. This is demonstrated in the following experiment:

A linearly polarized 300 Watt beacon transmitter emits a carrier signal at a frequency of 7 $\mathrm{MHz}$, which is below the critical frequency of the F2-layer (foF2) during the experiment on 8 to 10 March 2014. The strength of the beacon signal, reflected by the ionosphere, is measured at $110 \mathrm{~km}$ distance using a measurement receiver. Both characteristic waves are received separately on a dual polarization Turnstile antenna [Kraus, 1988, pp. 726-729] and their strength is recorded during a 48-hour period. A Rohde \& Schwarz FSMR26 measurement receiver is used with $100 \mathrm{~Hz}$ bandwidth, with a noise floor of $-138 \mathrm{dBm}(-31 \mathrm{~dB} \mu \mathrm{V})$. 
Measurement uncertainty is $0.3 \mathrm{~dB}$ for $95 \%$ confidence. The measurement set-up is illustrated in Figure 8.3 and a photograph of the turnstile antenna is shown in Figure 8.4. A detailed description of the experiment and the dual circular polarization antenna can be found in Section 7.3. The signal strength on the RHCP and LHCP antennas, measured during both measurements days, is shown in Figures 8.5 and 8.6.

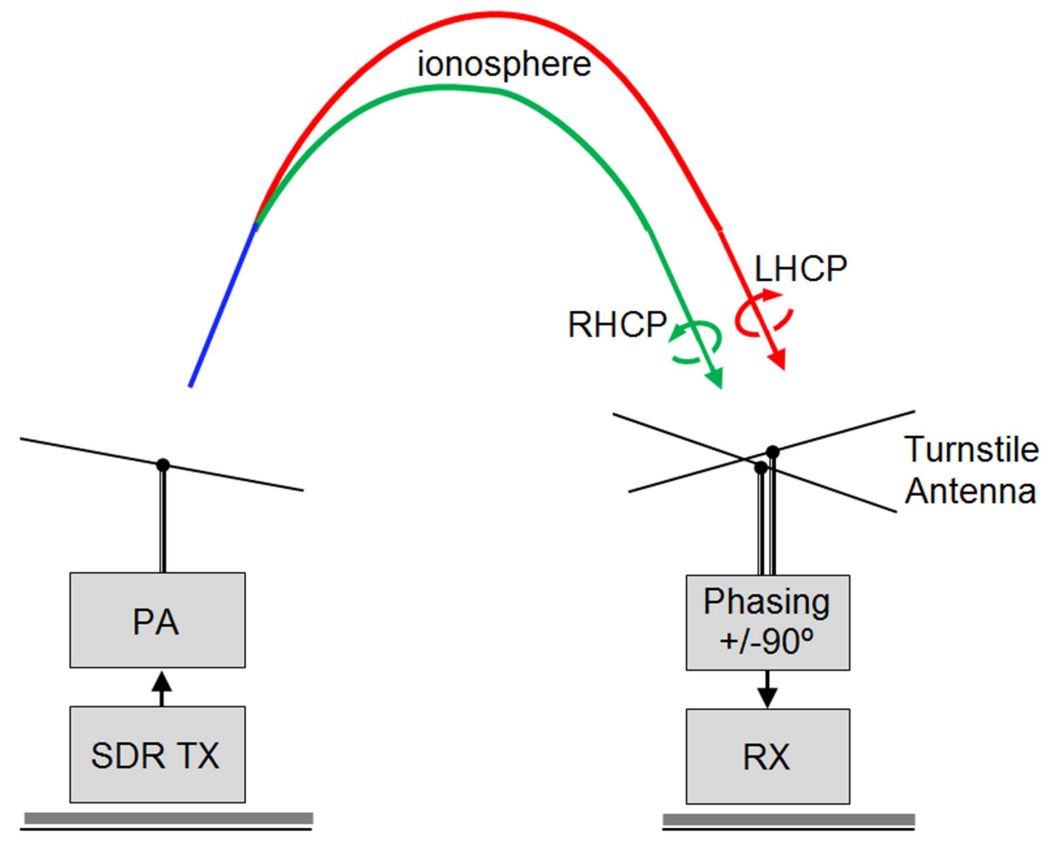

Figure 8.3 Measurement set-up of the NVIS characteristic diversity experiment. The signal of a linearly polarized transmitter is received on a dual circular polarization antenna at $110 \mathrm{~km}$ distance. SDR = Software Defined Radio.

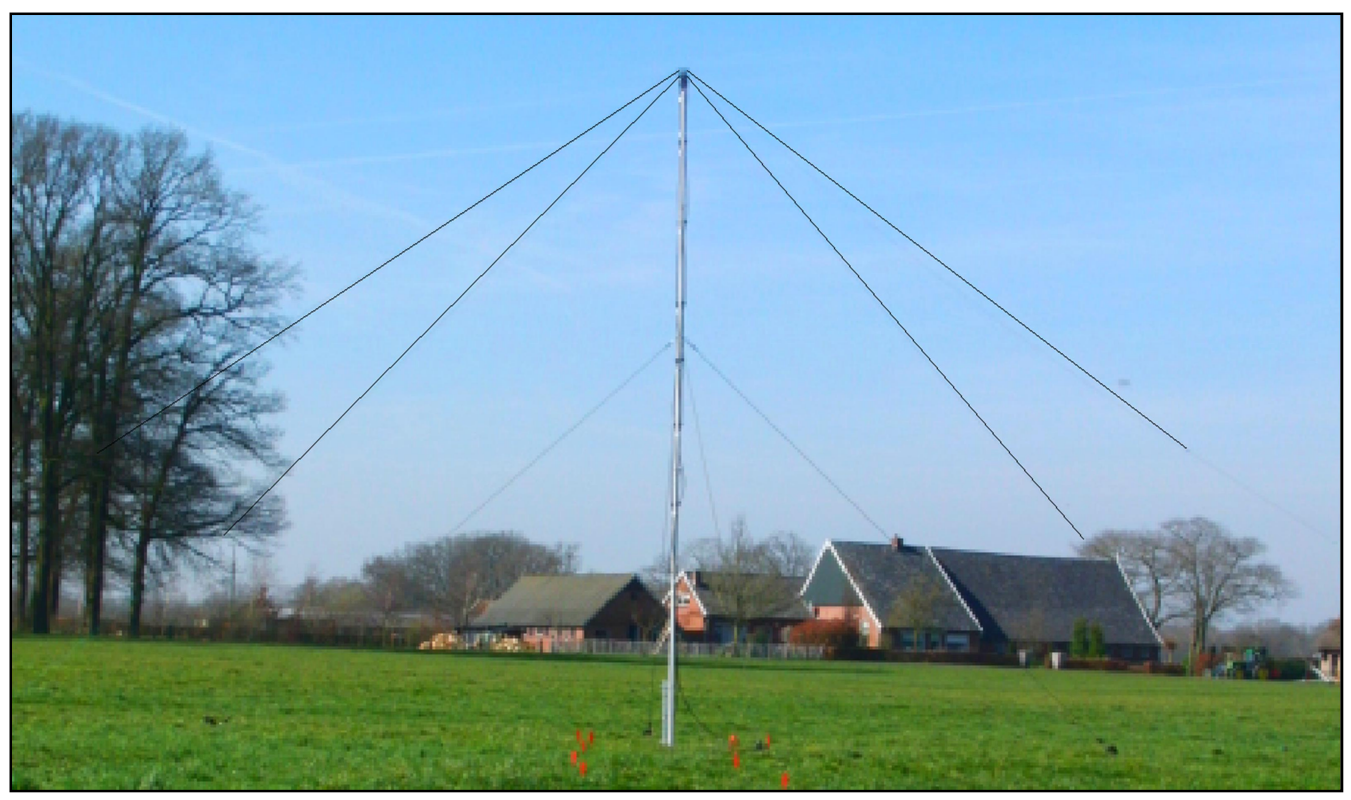

Figure 8.4 The turnstile antenna used in the experiments. Inverted Vee half-wave dipole elements are used. Apex height is 8.5 meters, end height is 2.5 meters. 

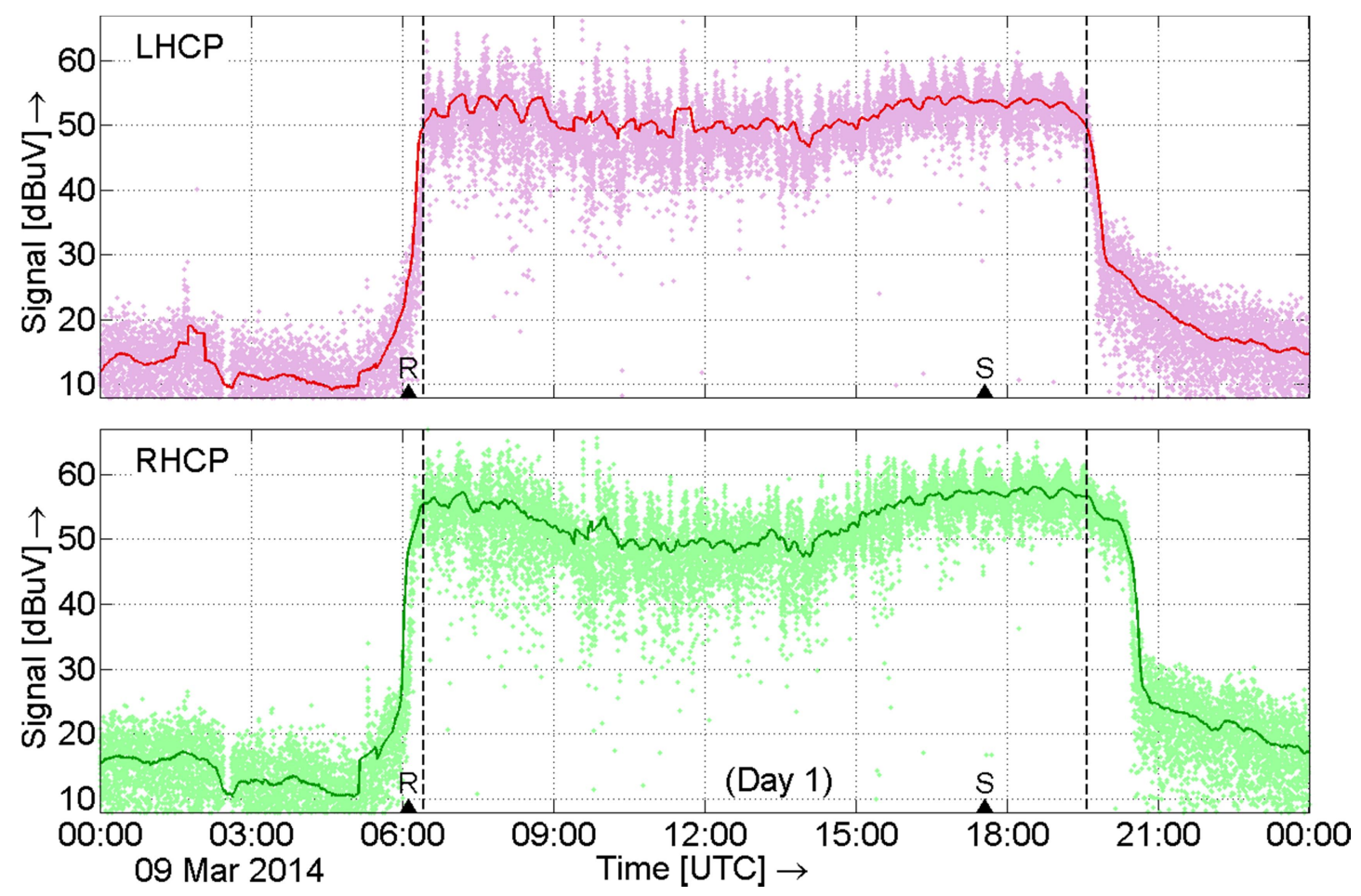

Figure 8.5 Measured power output of the RHCP (green) and the LHCP antenna (red) during the first measurement day (9 March 2014). A floating 20-minute average has been added as a solid line. Sunrise (R) at ground level is at 06:03 UTC, sunset (S) at 17:27 UTC.
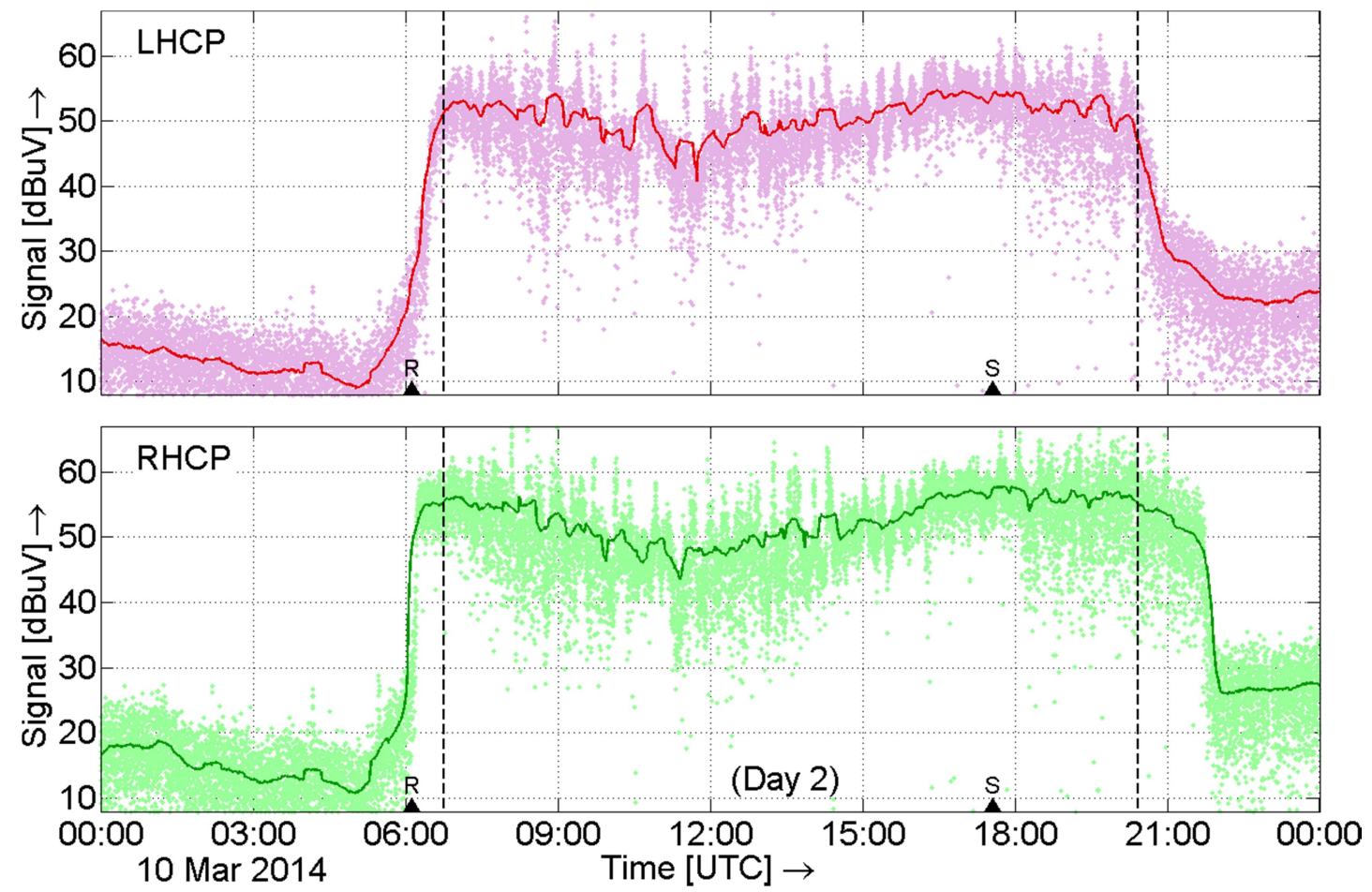

Figure 8.6 Measured signal strength on the RHCP (green) and the LHCP antenna (red) during the second measurement day (10 March 2014). A floating 20-minute average has been added as a solid line. Sunrise (R) at ground level is at 06:01 UTC, sunset (S) at 17:29 UTC. 
Sunrise at ground level is around 06:03 UTC, sunset is around 17:28 UTC. On both days NVIS propagation is present between approximately 06:00 to 21:00 UTC. The raw measurement samples show the short-term variation (fading) of the signal. A floating 20-minute average has been added as a solid line, to show the slower variation of the (mean) NVIS propagation.

\subsection{REDUCTION OF THE REQUIRED FADING MARGIN}

Zooming in on a portion of the measurements on 10 March 2014 from 12:05-12:55 UTC, Figure 8.7 shows the multipath fading in more detail, with minima that are $20-30 \mathrm{~dB}$ or more below the mean signal strength. Because of these minima, a large fading margin is needed to prevent drop-outs in the communication. Figure 8.7 also shows that the minima on the RHCP and the LHCP antenna do not coincide.

Therefore, if the output of each dual-polarization antenna is connected to a receiver, only one of the receivers will suffer signal loss during such a short fading minimum, while the other keeps receiving the signal at a much higher level. Consequently, if a selection mechanism is added to ensure that the receiver with the highest signal level is dynamically selected, a much lower fading margin is needed.

To estimate the reduction of the required fading margin when using this Characteristic Wave Diversity concept, the cumulative distribution of the measurements is calculated for each day. Only the intervals in which both characteristic waves can be received via NVIS propagation are used. On the first day that interval runs from 06:25 UTC to 19:35 UTC; on the second day it runs from 06:45 to 20:25 UTC. Figures 8.8 and 8.9 show the probability that the received signal URX is lower than the value UX for these two intervals.

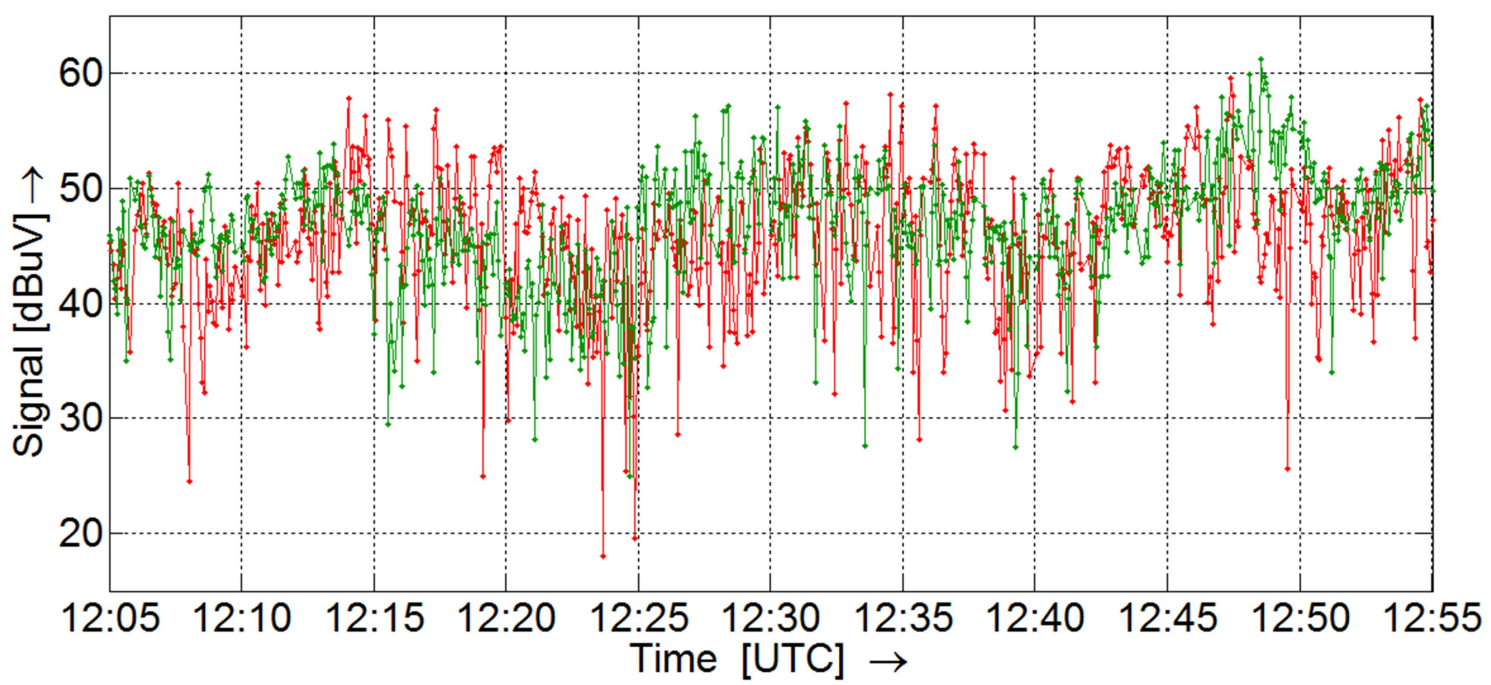

Figure 8.7 Detail from the measurements, showing that the deep fading minima on the RHCP (green) and the LHCP antenna (red) do not coincide. 
The green curve represents the RHCP signal, the red curve represents the LHCP signal and the blue curve shows the result of Characteristic Wave Diversity. The probability distributions of the LHCP and RHCP signals are slightly different, and vary from day to day.

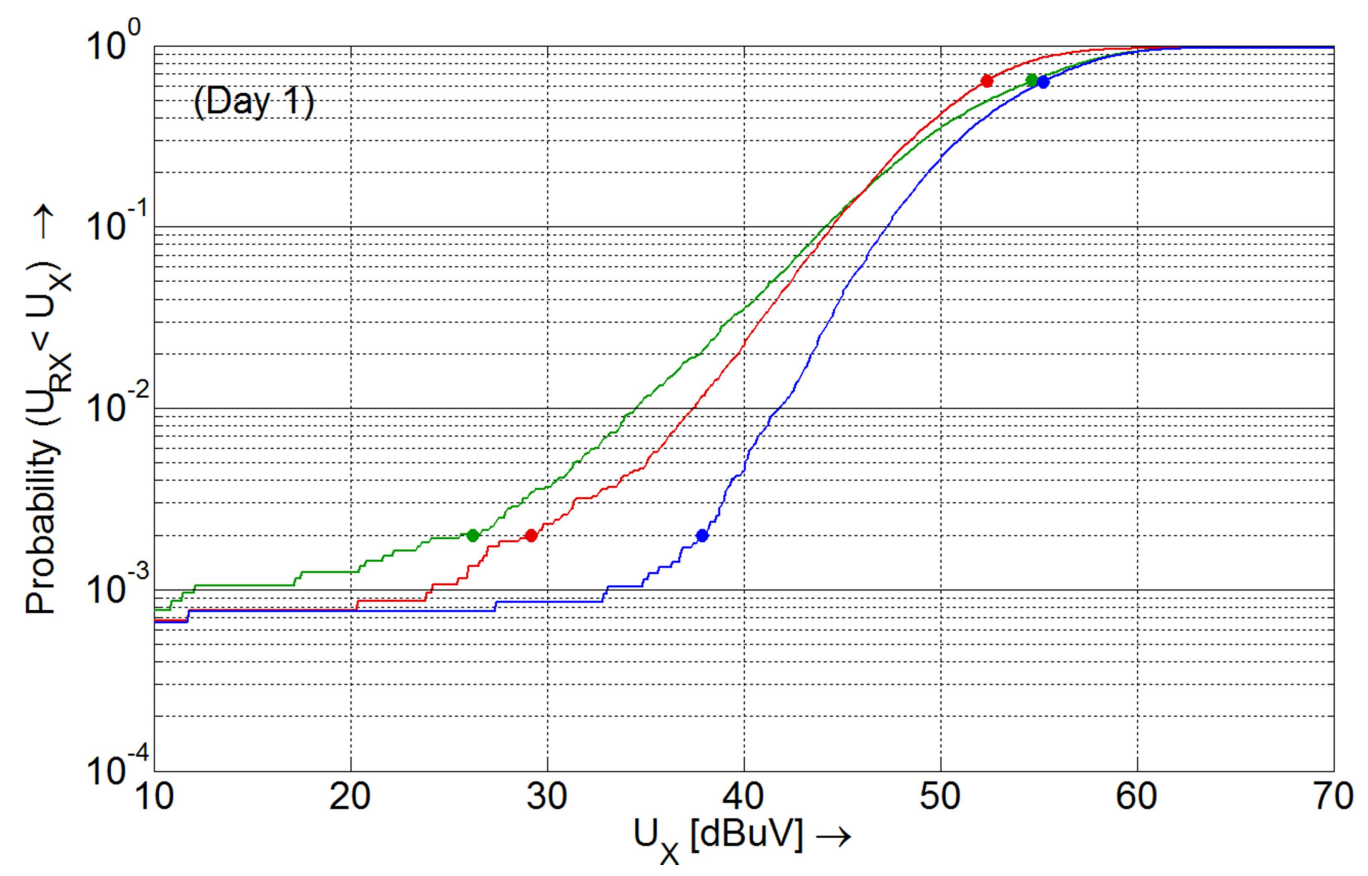

Figure 8.8 Cumulative distribution of the signal strength samples measured on 9 March 2014 (Day 1) on the RHCP antenna (green), on the LHCP antenna (red) and when using Characteristic Wave Diversity (blue). Dots are added to show the mean power and the $2.10^{-3}$ probability value.

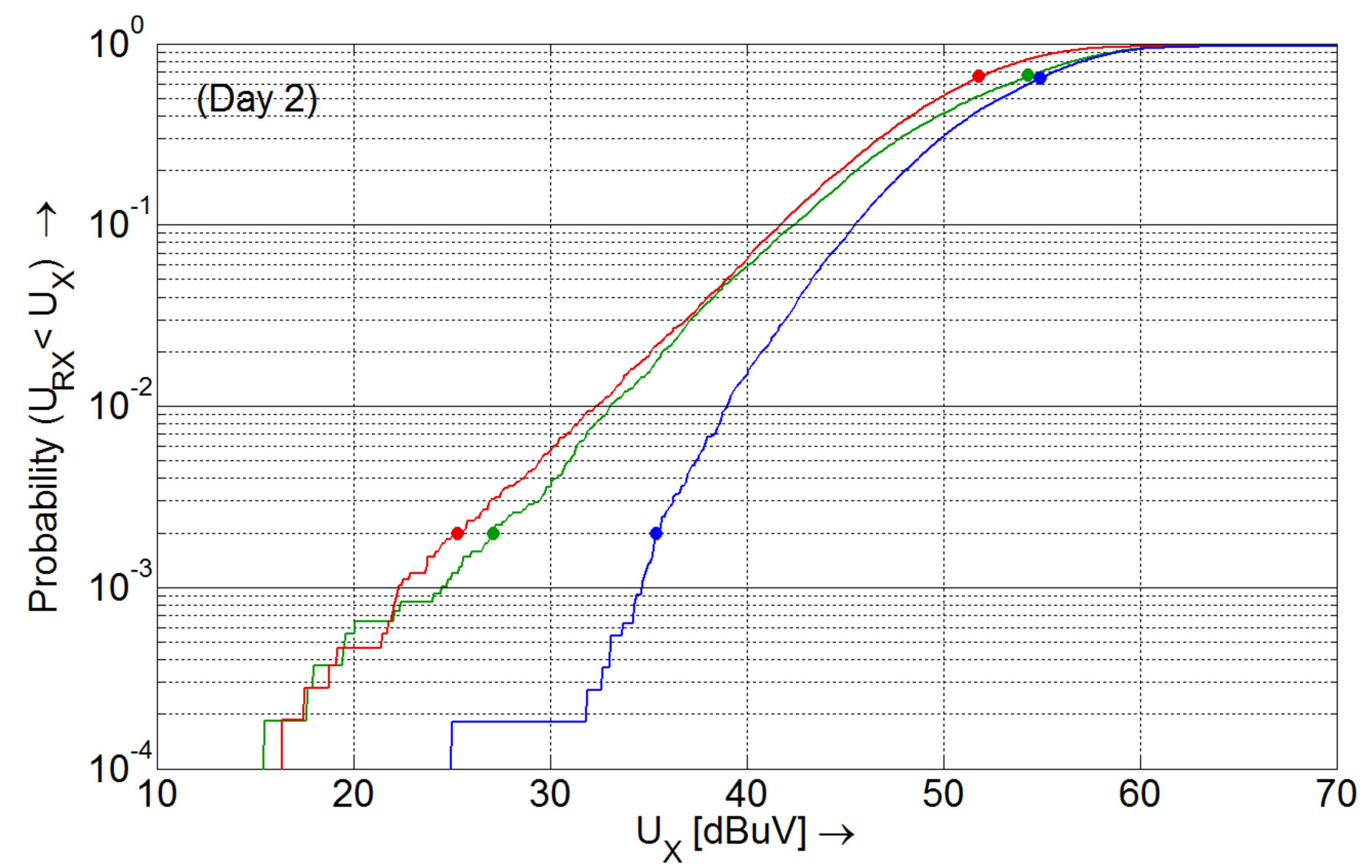

Figure 8.9 Cumulative distribution of the signal strength samples measured on 10 March 2014 (Day 2) on the RHCP antenna (green), on the LHCP antenna (red) and when using Characteristic Wave Diversity (blue). Dots are added to show the mean power and the $2.10^{-3}$ probability value. 
Table 8.1 Fading Margin for RHCP, LHCP and Diversity.

\begin{tabular}{|c|c|c|c|c|c|c|}
\hline \multirow{2}{*}{ Day } & \multicolumn{3}{|c|}{ RHCP } & \multicolumn{3}{|c|}{ LHCP } \\
\hline & $U_{R M S}$ & $U_{0.2 \%}$ & $F M^{a}$ & $U_{R M S}$ & $U_{0.2 \%}$ & $F M^{a}$ \\
\hline 1 & 54.7 & 26.2 & 28.5 & 52.4 & 29.2 & 28.4 \\
\hline \multirow[t]{2}{*}{2} & 54.3 & 27.1 & 27.2 & 51.8 & 25.3 & 29.5 \\
\hline & [dBuV] & {$[\mathrm{dBuV}]$} & [dB] & {$[\mathrm{dBuV}]$} & {$[\mathrm{dBuV}]$} & [dB] \\
\hline \multirow{2}{*}{ Day } & & & & \multicolumn{3}{|c|}{ Char. Wave Diversity } \\
\hline & & & & $\boldsymbol{U}_{R M S}$ & $U_{0.2 \%}$ & $F M^{a}$ \\
\hline \multirow{3}{*}{$\begin{array}{l}1 \\
2\end{array}$} & & & & 55.2 & 37.9 & 17.3 \\
\hline & & & & 54.9 & 35.4 & 19.5 \\
\hline & & & & {$[\mathrm{dBuV}]$} & {$[\mathrm{dBuV}]$} & {$[\mathrm{dB}]$} \\
\hline
\end{tabular}

For our purpose, we define the required fading margin as the ratio between the mean signal strength (URMS) over the entire interval and the signal strength below which the measured signal falls $0.2 \%$ of the time (U0.2\%). Both values are marked in the graphs with a dot on the curves. The corresponding values are summarized in Table I, as well as the required fading margins (FM) that are calculated from them.

The total number of measurements is slightly over 10.000 for each day. On the second day a larger number of deep fades (low signal levels) is seen. In Figure 8.8, due to the limited number of samples, the accuracy becomes low for probabilities below $2 * 10-4$. In Figure 8.9 this is the case for probabilities below $8 * 10-4$. Consistent values are obtained for both days for a probability of $0.2 \%$. According to [Davies, 1990, p. 237] the short-term ionospheric fading has approximately Rayleigh distribution, while a longer interval will show log-normal probability distribution. However, the Rayleigh distribution is still visible in the histogram of our measurement data, which holds the middle in between a lognormal and a Rayleigh distribution.

\subsection{DISCUSSION AND CONCLUSION}

From Table I we see that the required fading margin (FM) on a single RHCP or LHCP antenna is between 27.2 and $29.5 \mathrm{~dB}$, which is very large. With Characteristic Wave Diversity the required fading margin reduces to a value between 17.3 and $19.5 \mathrm{~dB}$. The improvement on day 1 is approximately $11.1 \mathrm{~dB}$, the improvement on day 2 is between 7.7 and $10.0 \mathrm{~dB}$. Implementation of this concept in an Emergency Communications Center would improve the communication with field workers that are limited to improvised antennas and low power transmitters. In adverse conditions the link can be maintained with 7.7 to $11.1 \mathrm{~dB}$ less transmitter power, which is a substantial improvement. No modification on the transmitter side of the link is required. 
The improvement given is relative to reception on a single circularly polarized NVIS-antenna. As was stated in Section 8.3, a single circularly polarized antenna is thought to be less sensitive to polarization fading than a linearly polarized antenna, which implies that the improvement of Characteristic Wave Diversity relative to reception on a linearly polarized antenna would be greater still. This was not investigated in this experiment, but will be subject to further research.

\subsection{FUTURE RESEARCH}

In these measurements alternating left hand and right hand circular polarization was obtained using a phasing network switched with coaxial relays, which limited the measurement speed to 2.5 seconds per sample. Consequently the number of measurements per time interval was insufficient to investigate lower probabilities $\left(<10^{-3}\right)$ or to investigate shorter time intervals and the variation of the fading margin over the day.

Also a direct comparison of the fading margin on a linearly polarized antenna, a single circularly polarized antenna and Characteristic Wave Diversity is desirable. For such a comparison, simultaneous sampling of the antennas would be preferred over alternating acquisition of samples as is used here, as this better represents the practical application. Additional experiments are planned for that purpose. Using a dual channel coherent receiver with a sampling speed meeting the Nyquist criterion, coherent samples can be obtained on two perpendicular dipoles. From these samples, orthogonal linear and orthogonal circular polarizations can be calculated in the digital domain, which can be used to compare the fading margins for identical NVIS propagation intervals. The associated measurement set-up is depicted in Figure 8.10.

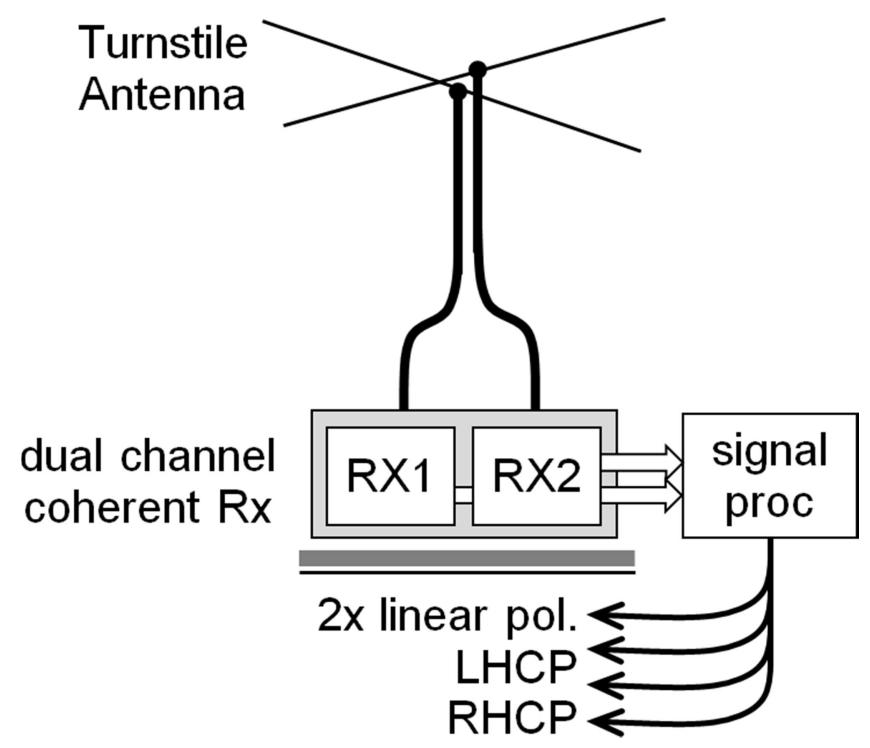

Figure 8.10 Experimental set-up for planned experiments, using a dual-channel coherent receiver to simultaneously monitor dual orthogonal linear and dual orthogonal circular polarizations. 
A more detailed analysis of the measured fading pattern such as in [ITU-R Rec. P.1407-5, 2013] could not be performed with present measurements due to the low sampling rate, but is intended in future experiments using higher sampling speeds. 


\section{Discussion and conclusions}


B. A. Witvliet

Interaction of Antenna and Propagation Mechanism 


\subsection{RÉSUMÉ}

In Chapter 2, an overview is given of the NVIS research field, encompassing NVIS history, antennas, propagation, channel characteristics, and modulation and coding for the NVIS channel. The annotations in that Chapter provide a comprehensive library of the NVIS research field. Research "white spots" are identified and research questions and subquestions concerning antennas and propagation have been formulated in Chapter 3. To answer these questions, a number of experiments have been designed and the results are presented in the subsequent Chapters. The text in Chapters 4 to 8 has been published in peer reviewed scientific publications.

In Chapter 4, the relationship between elevation angle and distance is shown to be dependent on frequency and sunspot number. It is also shown by measurement that the dominance of NVIS propagation mechanism over ground wave propagation starts at a short distance from the transmitter $(20 \mathrm{~km}$ at $7 \mathrm{MHz})$.

Also in Chapter 4, the adaptation of the NVIS antenna to the propagation mechanism is discussed. The transmit antenna must be optimized for maximum gain by concentrating all transmitted energy in the elevation angles that contribute to NVIS propagation. It is also shown that the presence of HF background noise (radio noise) requires a receive antenna that favors elevation angles used in NVIS while at the same time suppressing radio noise arriving from other angles. The results on reception, however, do not fully agree with the simulated values obtained when assuming uniform angular distribution of incoming radio noise. This triggered additional research into radio noise measurement antennas, presented in Chapter 5, showing the preference of a few popular antennas for a particular polarization and for specific directions.

New empirical results concerning specific aspects of the NVIS propagation mechanism - the characteristic wave propagation and the 'Happy Hour' propagation interval - are presented in Chapter 6 and 7. Measurements confirming its possible application in NVIS diversity and MIMO systems are presented in Chapter 8.

Three research questions and 15 subquestions were formulated in Chapter 3 . They cover the topics NVIS propagation, NVIS antennas and the interaction between the antennas and the propagation mechanism. These research questions will be discussed in the following Sections.

\subsection{DISCUSSION ON NVIS PROPAGATION}

Understanding the mechanism that underlies NVIS propagation is crucial for any attempt to improve the interface between the antenna and the propagation mechanism. Empirical data is very important in propagation research, and this is especially true when propagation in complex media such as the ionosphere is studied. The empirically obtained data produces a 
better insight into optimization goals and possible solutions. The first research question was formulated as:

Question 1: How does the NVIS propagation mechanism function, and what parameters of this mechanism are important for NVIS telecommunication system optimization?

The following subquestions were formulated:

Subquestion 1-1: Is it possible to realize a continuous coverage area of $400 \times 400 \mathrm{~km}$ using a single 100 Watt HF base station?

Subquestion 1-2: $\quad$ What frequencies should be used and what propagation mechanism is involved?

Subquestion 1-3: $\quad$ What is the role of ground wave propagation?

Subquestion 1-4: $\quad$ How much power is needed to realize coverage in the entire coverage area for at least $16 \mathrm{kbps}$ throughput in a $3 \mathrm{kHz}$ bandwidth?

Subquestion 1-5: What is the influence of multipath fading on the required link budget?

Subquestion 1-6: $\quad$ What is the relationship between the elevation angle of the transmitted waves and the distance covered? What other parameters are involved in this relationship?

Subquestion 1-7: $\quad$ What role does antenna polarization play in NVIS propagation?

\subsubsection{Subquestions on NVIS propagation}

The subquestions will be discussed first, with reference to the relevant Sections of this thesis. After that the main question is discussed.

Subquestion 1-1: Is it possible to realize a continuous coverage area of $400 \times 400 \mathrm{~km}$ using a single 100 Watt HF base station?

It was explained in Section 4.1 and 4.2 that, with proper frequency selection and a suitable antenna, a continuous coverage from the transmitter up to a $200 \mathrm{~km}$ radius can be realized. A single 100 Watt base station will provide 35-55 dB signal-to-noise ratio (SNR) in $3 \mathrm{kHz}$ bandwidth in the entire coverage area.

Subquestion 1-2: What frequencies should be used and what propagation mechanism is involved?

As was shown in paragraph 4.2.1 and illustrated in Figure 4.3, the operating frequency must be below the critical frequency of the reflecting ionospheric layer to avoid the creation of a skip zone, a circular zone around the transmitter in which there is no skywave coverage. The frequency selection depends on the time of the day, the season and the year in the solar 
cycle. For daytime propagation on mid-latitude locations, operating frequencies range from 3 to $10 \mathrm{MHz}$.

\section{Subquestion 1-3: What is the role of ground wave propagation?}

The role of the ground wave component when covering an area of $400 \times 400 \mathrm{~km}$ is small. As shown by measurements in paragraph 4.3.2 the dominance of NVIS (skywave) over groundwave propagation started at $20 \mathrm{~km}$ from the transmitter when a frequency of $7 \mathrm{MHz}$ was used. At $3.5 \mathrm{MHz}$ this was $40 \mathrm{~km}$. Up to this distance signals arriving via groundwave and skywave may interfere, causing additional multipath fading.

Subquestion 1-4: How much power is needed to realize coverage in the entire coverage area for at least $16 \mathrm{kbps}$ throughput in a $3 \mathrm{kHz}$ bandwidth?

For an answer to this question we may look the signal strength values measured during the experiments described in Chapters 4, 6 and 7.

In Section 4.5 an 850 Watt transmitter yielded approximately $50 \mathrm{~dB} \mu \mathrm{V}$ average signal strength in the morning, and $60 \mathrm{~dB} \mu \mathrm{V}$ in the afternoon. In Sections 6.3 and 6.4 an 800 Watt transmitter yielded $56 \mathrm{~dB} \mu \mathrm{V}$, and in Sections 7.3 and 7.4 a 300 Watts transmitter yielded between 45 and $58 \mathrm{~dB} \mathrm{~dB} \mu \mathrm{V}$. In each of these examples half-wave dipole antennas were used on both ends of the path. When the transmit power is normalized to $1 \mathrm{Watt}$, the average signal strength is between 20 and $33 \mathrm{~dB} \mu \mathrm{V}$.

All three measurements were performed in a rural area, and the measured radio noise levels were around $-20 \mathrm{~dB} \mu \mathrm{V}$ in $30 \mathrm{~Hz}$ bandwidth (Figure 4.22) and $-10 \mathrm{~dB} \mu \mathrm{V}$ in $100 \mathrm{~Hz}$ bandwidth (Figure 7.20). This corresponds with 0 to $5 \mathrm{~dB} \mu \mathrm{V}$ in $3 \mathrm{kHz}$ bandwidth.

So for a 1 Watt ( $0 \mathrm{dBW}$ ) transmitter the expected SNR would be between 15 and $33 \mathrm{~dB}$. As was discussed in Section 2.6.1, Hoult [2000] demonstrated data transfer speeds of up to 16 kbps in a $3 \mathrm{kHz}$ channel at an SNR > $27 \mathrm{~dB}$ using NVIS propagation. From the above values we see that this SNR will be realized with an RF output power between $-6 \mathrm{dBW}$ ( $0.25 \mathrm{Watt}$ ) and $12 \mathrm{dBW}$ (16 Watts). These values are calculated in the absence of interference and manmade noise.

Subquestion 1-5: What is the influence of multipath fading on the required link budget? As is shown in Sections 8.4 and 8.5 by measurement, a fading margin of 27 to $30 \mathrm{~dB}$ is required. This margin can be reduced by 8 to $11 \mathrm{~dB}$ by using Characteristic Wave Diversity. 
Subquestion 1-6: What is the relationship between the elevation angle of the transmitted waves and the distance covered? What other parameters are involved in this relationship?

The relationship between the elevation angle of the transmitted waves and the distance covered depends on the virtual reflection height in the ionosphere and whether or not diffraction occurs in lower layers that are penetrated. This is illustrated in Figure 4.7 in paragraph 4.3.1. The virtual reflection height and the amount of refraction in lower layers depend upon the electron density profile of the ionosphere and the frequency of the incoming wave. Consequently the relationship between elevation angle and distance is frequency dependent and changes slowly with the progress of the solar cycle. Figures 4.5 and 4.6 of paragraph 4.3.1 show this relationship in graphical form for a solar cycle minimum and a moderate solar cycle maximum. Typical elevation angles range from $70^{\circ}$ to $90^{\circ}$ for a coverage area with $150 \mathrm{~km}$ radius, i.e. approximately 300 × $300 \mathrm{~km}$ area.

\section{Subquestion 1-7: What role does antenna polarization play in NVIS propagation?}

As discussed in paragraph 6.1.1 and Sections 7.2 and 8.2, linearly polarized waves that are transmitted upwards and enter the ionosphere split into two circularly polarized waves of opposite sense. Only these circularly polarized 'characteristic waves' propagate in the ionosphere and both waves travel a different path and suffer different delays and attenuations. Leaving the ionosphere on their downward path, the characteristic waves interfere and produce a complex multipath fading profile, both in amplitude and in polarization. Experiments described in Sections 6.3 and 7.3 prove that the characteristic waves have little interaction in the ionosphere and can be received separately with more than $25 \mathrm{~dB}$ isolation. Using the separately received characteristic waves in a diversity system, the depth of the fading minima can be reduced by 8 to $11 \mathrm{~dB}$, and 8 to $11 \mathrm{~dB}$ less transmitter power is needed for the same quality NVIS radio link. This is demonstrated empirically in Sections 8.3 and 8.4 .

\subsubsection{Research question on NVIS propagation}

From the answers to the subquestions given above, an answer to our main research question on NVIS propagation can be formulated.

Question 1: How does the NVIS propagation mechanism function, and what parameters of this mechanism are important for NVIS telecommunication system optimization?

NVIS propagation is possible on frequencies between approximately 3 and $10 \mathrm{MHz}$. It involves upward and downward radio waves with steep elevation angles. These elevation angles depend on frequency, distance and the solar cycle, but typical elevation angles are from $70^{\circ}$ to $90^{\circ}$ for a coverage area with a $150 \mathrm{~km}$ radius (resulting in a $300 \times 300 \mathrm{~km}$ coverage area). NVIS is more power efficient than ground wave propagation for distances greater than $20 \mathrm{~km}$ on $7 \mathrm{MHz}$ and or $40 \mathrm{~km}$ on $3.5 \mathrm{MHz}$. 
In NVIS, upward radio waves are split into left hand circularly polarized (LHCP) and right hand circularly polarized (RHCP) components when entering the ionosphere, and downward RHCP and LHCP waves leave the ionosphere on their way to the receiver. The isolation of the waves traveling in the ionosphere exceeds $25 \mathrm{~dB}$. The ionospheric propagation channel suffers from multipath fading, which is partly caused by interference between the downgoing RHCP and LHCP waves. The fading minima are $27 \mathrm{~dB}$ to $30 \mathrm{~dB}$ below the average signal power, therefore an equivalent fading margin is required for link sustenance. Ambient electromagnetic noise (radio noise) is a limiting factor in the reception of NVIS signals. This noise arrives at the antenna from various spatial angles via a number of propagation mechanisms.

Considering the above - if we limit ourselves to antennas and propagation - elevation angles, polarization, fading and noise are the most important parameters to consider in NVIS telecommunication system optimization.

\subsection{DISCUSSION ON NVIS ANTENNAS}

The answers of research question 1 may be used as input to optimize NVIS transmit and receive antennas. While NVIS antenna optimizations have been studied before, the differentiation between transmit and receive properties has not received sufficient attention, and other aspects of matching the antenna to the propagation mechanism may have been missed.

Question 2: How can we optimize the NVIS antenna to (a) produce the strongest signal across the coverage area, and (b) to realize the greatest signal-to-noise ratio on reception of signals from that coverage area?

The following subquestions were formulated:

Subquestion 2-1: Which antenna parameters must be considered for optimization?

Subquestion 2-2: Is there a difference between transmit and receive antenna optimization?

Subquestion 2-3: $\quad$ What role does electromagnetic background noise (radio noise) play in reception?

Subquestion 2-4: How does the angular distribution of radio noise influence antenna optimization? 


\subsubsection{Subquestions concerning NVIS antennas}

Subquestion 2-1: Which antenna parameters must be considered for optimization?

Section 4.4 discusses antenna optimization for communication via NVIS propagation. The antenna performs best when all radiation is concentrated in the direction of the spatial angles used in NVIS propagation. To ensure the entire NVIS coverage area is served, NVIS Antenna Directivity is defined as the average directivity calculated over all NVIS angles. The theoretical maximum gain that can be achieved is $15.2 \mathrm{dBi}$, as is shown in paragraph 4.4.3.

Subquestion 2-2: Is there a difference between transmit and receive antenna optimization?

There is a distinct difference between transmit and receive antenna optimization. On transmission both the directivity and the radiation efficiency of the antenna is important. Therefore the antenna gain, being the product of antenna efficiency and directivity, is to be optimized for transmission. Antenna efficiency does not influence reception, unless the receiver sensitivity is very poor. A lower efficiency will reduce both the wanted signal and the ambient electromagnetic noise (radio noise), leaving the signal-to-noise ratio (SNR) unchanged. Therefore only the antenna directivity is to be optimized on reception. This distinction in antenna optimization for transmission and reception is demonstrated by calculation and with antenna simulations in paragraph 4.4 .2 and 4.4 .3 , and by experiment in paragraphs 4.5.3 and 4.5.4.

Subquestion 2-3: What role does electromagnetic background noise (radio noise) play in reception?

In the lower $\mathrm{HF}$ frequency range (3-10 $\mathrm{MHz}$ ) radio noise is dominant over the receiver noise, and an increase in radio noise will therefore decrease the ability to receive weak signals.

Predicted absolute levels of radio noise are important for radio system design and the graphs in ITU-R Recommendation P.372 [2013] are often referred to. The values in this recommendation are obtained using a Short Lossless Vertical Monopole, which has reduced sensitivity to skywave radio noise and responds to vertical polarization only, as shown in paragraph 5.3.1, making application to NVIS reception unreliable.

Subquestion 2-4: How does the angular distribution of radio noise influence antenna optimization?

The reception will improve when the amount of received radio noise is reduced through the use of an antenna that - by its radiation pattern - suppresses signals from the angular directions from which most radio noise arrives. Knowledge of these angular directions [Coleman, 2002] is therefore important for optimum antenna design. This is illustrated in 
paragraph 4.5.4, where results from a receive antenna optimization assuming uniform angular distribution are found to differ from empirically obtained values.

\subsubsection{Research question on NVIS antennas}

From the above, an answer to our main research question on NVIS antennas can be formulated.

Question 2: How can we optimize the NVIS antenna to (a) produce the strongest signal across the coverage area, and (b) to realize the greatest signal-to-noise ratio on reception of signals from that coverage area?

(a) The strongest signal in the coverage area can be realized by optimizing the transmit antenna for the greatest NVIS Antenna Gain, i.e. the greatest average antenna gain over the elevation angles that contribute to NVIS propagation to the coverage area.

(b) The greatest signal-to-noise-ratio can be achieved by optimizing the ratio of the average antenna gain over the elevation angles that contribute to NVIS propagation from the coverage area to the receive antenna, divided by the antenna gain over the elevation angles from which most radio noise is received. This optimization requires knowledge of the propagation of radio noise from its sources to the receiver and information on the expected angular distribution of the dominant radio noise sources.

\subsection{DISCUSSION ON THE INTERACTION OF ANTENNA AND PROPAGATION}

If interaction between the antenna and the propagation mechanism is negligible, the problem formulated in research question 2 simplifies to a simple antenna optimization. If this interaction is not negligible, a more holistic approach is needed, and the antenna and propagation mechanism must be regarded as a hybrid system.

Question 3: How important is the interaction between NVIS antenna and NVIS propagation mechanism?

The following subquestions were formulated:

Subquestion 3-1: In what ways do the transmit antenna properties change the propagation of the radio waves in the ionosphere?

Subquestion 3-2: In what ways must the receive antenna be adapted to the ionospheric radio wave propagation?

Subquestion 3-3: Must we consider the combination of antenna and propagation mechanism as a hybrid system with a mutual dependency?

Subquestion 3-4: How can knowledge of the NVIS antenna-propagation interaction be used to reduce the necessary transmit power or to increase the data throughput? 


\subsubsection{Subquestions concerning the interaction of antenna and propagation}

Subquestion 3-1: In what ways do the transmit antenna properties change the propagation of the radio waves in the ionosphere?

A linearly polarized transmit antenna will excite both characteristic waves in the ionosphere that will independently propagate in the ionosphere and cause multipath and polarization fading when recombining at the receive antenna, as was described in Section 8.2. When a single circularly polarized antenna is used, only one of the characteristic waves propagates in the ionosphere. As a consequence there is no polarization fading and the downward wave has circular polarization. When two orthogonal circularly polarized waves are transmitted, these waves will travel independently through the ionosphere. They can even be modulated independently and, if receive antennas with matching polarization are used, both data streams can be demodulated separately or in a MIMO arrangement. This arrangement, proposed in Section 7.6, could potentially double the data transfer on the same frequency.

The vertical radiation pattern of the transmit antenna will also influence the propagation in the ionosphere. As was discussed in Section 4.3, only high elevation angles contribute to NVIS propagation, and an NVIS transmit antenna that concentrates all transmit power into high elevation angles produces stronger signals in the coverage area. Power transmitted at lower angles will cause long distance propagation and hence unintended interference to other radio communication systems located further away. The vertical radiation pattern may also be designed to favor either E-layer (intermediate angle) or F-layer (high angle) reflection, preventing or reducing multipath propagation that would occur when both layers reflect the signal towards the intended destination.

Subquestion 3-2: In what ways must the receive antenna be adapted to the ionospheric radio wave propagation?

As with the transmit antenna, a receive antenna with a vertical radiation pattern favoring the higher elevation angles will reduce interference from other radio communication systems located further away. Additionally, the SNR can be improved when the antenna diagram suppresses angular directions from which skywave radio noise arrives, as was discussed in paragraph 4.4.3. Reduction of radio noise is not only important in urban areas, where man-made noise is high. Also in quiet rural areas, the remaining radio noise from natural sources, as depicted in Figure 5.2, defines the reception limit.

Furthermore, by adapting the polarization of the receive antenna to the properties of the characteristic waves propagating in the ionosphere, e.g. by using a receive antenna with dual circular polarization, significant fading reduction can be achieved. This has been examined in Section 8.3 , and an 8 to $11 \mathrm{~dB}$ reduction of the power necessary to maintain the radio communication link was demonstrated. 
Subquestion 3-3: Must we consider the combination of antenna and propagation mechanism as a hybrid system with a mutual dependency?

There is substantial interaction between the propagation channel and the antenna system used. The characteristics of the propagation channel are influenced by the transmit and the receive antenna properties, as was discussed above answering subquestions 3-1 and 3-2. Similarly, the transmit and receive antenna can only be optimized by considering the properties of the propagation channel, e.g. the polarization of the characteristic waves (Section 7.2), the elevation angles involved (Section 4.3) and the angular distribution and polarization of radio noise (Section 5.2). Optimal solutions for NVIS radio communication systems must therefore consider the combination of antenna and propagation mechanism as a hybrid system for optimization.

Subquestion 3-4: How can knowledge of the NVIS antenna-propagation interaction be used to reduce the necessary transmit power or to increase the data throughput?

Considering the isolation measured in Section 7.5, two orthogonal radio links can be created on the same frequency by exciting and receiving the two characteristic waves. This potentially doubles the channel capacity, but requires a two-channel transmitter and a twochannel receiver. The antenna system may remain simple and compact, following the designs described in paragraph 7.3.2.

Alternatively the transmit end of the link could remain unchanged - a single transmitter with a linearly polarized antenna - while the receive end is modified as shown in Section 8.6 to provide simultaneous reception of both characteristic waves. The output of this antennareceiver system can be used for Characteristic Wave Diversity, which is discussed in Sections 8.3 and 8.4, and yields an 8 to $11 \mathrm{~dB}$ gain improvement in required link margin, which can be used for transmit power reduction or increased throughput.

\subsubsection{Research question on the interaction of antenna and propagation}

From the above, an answer to our research question on the interaction of antenna and propagation can be formulated.

Question 3: How important is the interaction between NVIS antenna and NVIS propagation mechanism?

There is significant interaction between antenna parameters and parameters of the ionospheric channel. For optimization of the NVIS telecommunication system, it is therefore important to consider antenna and propagation mechanism as a hybrid system that must be optimized for optimal power transfer, but also for favorable channel characteristics for modulation and decoding systems. Considering the antenna and propagation interaction is 
especially important when increased data throughput is sought. Diversity and MIMO systems can be enhanced by adapting the antenna to the characteristic waves in the ionosphere.

\subsection{DISCUSSION ON COMMON MISUNDERSTANDINGS}

The following commonly accepted misunderstandings (myths) concerning HF propagation in general and NVIS in particular have been identified in Section 3.3:

Assumption 1: The NVIS antenna must be suspended at low heights above ground to give best radiation towards the zenith and hence best NVIS performance.

Assumption 2: The signal-to-noise ratio (SNR) of an NVIS signal received on a dipole antenna suspended close to the ground is much better than the SNR on a dipole that is suspended higher above ground.

Assumption 3: Vertical whips on cars may be poor NVIS performers, but if you tilt the whip by 45 degrees, performance is restored.

Assumption 4: Short HF radio links - up to and maybe even beyond $100 \mathrm{~km}$ distance depend on ground wave propagation and require a vertically polarized antenna.

Assumption 5: At night, on frequencies where the ionosphere does no longer support NVIS, signals arrive via ground wave.

Assumption 6: Waves propagating through the ionosphere have linear polarization when launched from linearly polarized antennas.

These assumptions will be discussed below, with reference to the Sections of the thesis in which relevant research is described.

Assumption 1: The NVIS antenna must be suspended at low heights above ground to give best radiation towards the zenith and hence best NVIS performance.

As was shown by simulation in Section 4.4.2, and by measurement in Section 4.5.3, a horizontal half-wave dipole antenna yields 11 to $12 \mathrm{~dB}$ lower antenna gain when suspended at low height $(0.02 \lambda)$ than an identical antenna suspended at the optimum height of $0.19 \lambda$. While this has been empirically verified for farmland soil only, the simulations predict comparable results for other soil types. Therefore, if we define 'low' as 'lower than $0.05 \lambda$ ', assumption 1 is incorrect.

Assumption 2: The signal-to-noise ratio (SNR) of an NVIS signal received on a dipole antenna suspended close to the ground is much better than the SNR on a dipole that is suspended higher above ground. 
Simulations in Section 4.4.3 assuming a uniform spatial distribution of radio noise show very little variation of predicted SNR with height, with an optimum antenna height of $0.09 \lambda$ for farmland. However, verification measurements in Section 4.5.4 show that the optimum antenna height is higher ( $0.16 \lambda$ for farmland) and that the optimum is more pronounced than was expected from simulations. Both simulations and measurement disprove the assumption that a low antenna height is preferable for NVIS reception.

Assumption 3: Vertical whips on cars may be poor NVIS performers, but if you tilt the whip by 45 degrees, performance is restored.

The antenna gain towards the zenith of a vertical whip antenna on a car is - $35 \mathrm{dBi}$ [Hagn, 1970]. This rises $-32 \mathrm{dBi}$ when the vertical is tilted forward over the car, and to $-17 \mathrm{dBi}$ when the whip is tilted away from the vehicle, which is impractical while driving. The latter value is still $23.5 \mathrm{~dB}$ lower than a half wave dipole antenna at $0.19 \lambda$ (Figure 4.16, farmland), a substantial difference. Even a horizontal dipole at 1 meter $(0.02 \lambda$ at $5 \mathrm{MHz})$ above farmland, a height far below the optimum, still outperforms the tilted whip on a car by $12 \mathrm{~dB}$. So this assumption is incorrect.

Assumption 4: Short HF radio links - up to and maybe even beyond $100 \mathrm{~km}$ distance - depend on ground wave propagation and require a vertically polarized antenna.

This assumption was negated by the measurements described in paragraph 4.3.2. On $7 \mathrm{MHz}$ the dominance of NVIS (skywave) over groundwave propagation started at $20 \mathrm{~km}$ from the transmitter, at $3.5 \mathrm{MHz}$ this was $40 \mathrm{~km}$.

Assumption 5: At night, on frequencies where the ionosphere does no longer support NVIS, signals arrive via ground wave.

This assumption could not be fully disproven with the experiments contained in this thesis, but observations in paragraph 7.5.4 strongly suggest that the nighttime propagation is via scattering in the ionosphere or ground scatter at a distance that can still be reached by ionospheric reflection after NVIS propagation has ceased. Further experiments that we completed recently confirm this. A publication is in preparation.

Assumption 6: Waves propagating through the ionosphere have linear polarization when launched from linearly polarized antennas.

The polarization of waves propagating through the ionosphere has been studied in Chapters 6 and 7. A linearly polarized upward wave is returned as two orthogonal circularly polarized waves with opposite rotation sense. Even their combined field arriving at the receive antenna is not linearly polarized, but rather elliptically polarized with rapidly changing axial ratio and rotation sense. This assumption is incorrect. 


\subsection{CONTRIBUTIONS}

In this thesis the following contributions are made:

NVIS propagation, elevation angles and ground wave

- The relationship between elevation angle and coverage distance is established in Section 4.3 as a function of frequency and for low and high sunspot numbers. This relationship is confirmed by measurement.

- The dominance of NVIS over groundwave propagation is shown by measurement to start at very short distances: $20 \mathrm{~km}$ at $7 \mathrm{MHz}$ and $40 \mathrm{~km}$ at $3.5 \mathrm{MHz}$.

\section{Daytime and nighttime propagation}

- Using measurements, NVIS propagation is shown to be very efficient: one 100 Watt transmitter will cover a $400 \times 400 \mathrm{~km}$ area with 35-55 dB SNR in a $3 \mathrm{kHz}$ bandwidth.

- Nighttime propagation is observed above the critical frequency of the ionosphere, showing signal fluctuation similar to scattering and unlike ground wave propagation. This was observed in all experiments, and described in paragraph 4.5.3, Section 6.4 and paragraph 7.5.4. Signal levels are approximately $50 \mathrm{~dB}$ lower than daytime NVIS propagation on $7 \mathrm{MHz}$.

\section{Difference in antenna performance on transmission and reception}

- In section 4.4, the distinction in requirements between transmit and receive antennas is shown, involving the antenna diagram and the antenna efficiency.

- Two novel figures-of-merit are introduced in paragraph 4.4.2 defining transmit and receive antenna performance at the spatial angles that contribute to NVIS: 'NVIS Antenna Gain' and 'NVIS Antenna Directivity'.

- The measurement results presented in paragraph 4.5 .4 suggest that during daytime NVIS propagation, the spatial distribution of radio noise is not uniform.

\section{Antenna optimization and verification}

- The relationship between NVIS antenna performance and antenna height-aboveground is studied for several soil types using antenna simulations in Section 4.4. Transmit and receive antennas are investigated separately. Height optima are found, which are verified by measurement in Section 4.5.

- A novel method for in-situ comparison of antenna performance in the presence of real NVIS propagation is introduced in paragraph 4.5.1. Both transmission and reception performance are measured simultaneously. This method produces accurate results in the presence of multipath fading. 


\section{Characteristic wave propagation}

- The importance of characteristic wave propagation in NVIS has been demonstrated by measurement in Chapter 6.

- It has been demonstrated by measurements described in Chapter 7, that the polarization of the downward characteristic waves is nearly perfectly circular and that high isolation exists ( $>25 \mathrm{~dB}$ ) between both characteristic waves.

- The existence of a special propagation interval ('Happy Hour') at the onset and end of the daily NVIS propagation interval, in which only the extraordinary wave propagates, is demonstrated by measurement in Chapter 6 and 7. This phenomenon was predicted, but not observed, by McNamara [2008].

Characteristic wave diversity and MIMO

- Separate reception of both characteristic waves on an antenna with a footprint of only $0.5 \times 0.5$ wavelength footprint with an isolation of $25 \mathrm{~dB}$ has been demonstrated in Chapter 7. This is a substantial improvement compared to antenna correlation between 0.2 and 0.9 reported by other investigators using different antenna systems with much larger footprints [Gunashekar, 2009].

- In Chapter 8, characteristic wave diversity is shown to produce 8 to $11 \mathrm{~dB}$ reduction of the required fading margin, thereby reducing the necessary transmit power by the same amount.

\section{Radio noise measurement antennas}

- In Section 5.2, an overview is given of the propagation mechanisms involved in the distribution of HF radio noise.

- A novel method for the comparison of HF radio noise measurement antennas, with visualization of the predisposition of the antenna to polarization and spatial angle of the incoming waves is introduced in Section 5.3.

- In Sections 5.3.1 to 5.3.3 this method is used to compare the performance of three basic radio noise measurement antennas.

- In Section 5.4 a synthetic unpolarized omni-directional antenna system, consisting of two antenna elements and a dual channel receiver, is proposed for improved HF radio noise measurements in the presence of skywave propagation.

\subsection{CONCLUSION}

In this thesis a number of contributions is made that contribute to a better understanding of NVIS propagation and the interaction of NVIS antennas and the propagation mechanism. It is shown that isolated optimization of individual elements of an NVIS telecommunication system will not yield the optimum performance that can be achieved when the interaction between the system components is considered in the design. 
An optimization of half-wave dipole antennas with respect to antenna suspension height has been performed, both for transmission and reception, and the advantage of adapting the polarization of the antenna to the characteristic waves traveling in the ionosphere is demonstrated.

Besides these results, several methods for assessment by simulation and empirical verification of the performance of the antenna-propagation hybrid have been introduced and demonstrated, with sufficient detail for reproduction. These methods can be adopted by other investigators to solve similar verification problems involving ionospheric propagation. 


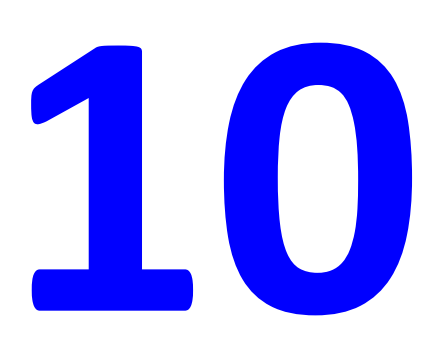

Topics for further research 
Interaction of Antenna and Propagation Mechanism 


\subsection{ONGOING RESEARCH}

At every journey's end, a new horizon calls. The research presented here contributed significantly to our insight in NVIS antennas and propagation. It also triggered new questions that we are eager to investigate. The experience gained with our previous experiments enables more advanced experiments, making these new horizons attainable. Planned research will be discussed in this section. Remaining research questions are discussed in Section 10.2.

\subsubsection{Path isolation experiments with dual circular polarization}

To prove that the characteristic wave isolation measured in Chapters 6 and 7 is present in the entire NVIS propagation interval and not only during the 'Happy Hour', an experiment was planned using an NVIS beacon transmitter transmitting alternatingly in LHCP and RHCP, while the receiver measures field strength alternatingly on an LHCP and an RHCP antenna. This experiment was successfully executed in December 2014 and showed that two channels were created through the ionosphere on the same frequency during the entire NVIS propagation interval with less than $-22 \mathrm{~dB}$ crosstalk. Remarkably, even when launching and receiving a single characteristic wave, multipath fading was observed. A publication is in preparation.

\subsubsection{Nighttime above-the-MUF propagation measurements}

During the experiments described in Chapters 4 and 6 , the beacon signal arrived with $>20 \mathrm{~dB}$ SNR at night, where no NVIS propagation was expected. The signal properties did not correspond with ground wave propagation. In parallel with the experiment described in paragraph 10.1.1, high speed high resolution spatial angle-of-arrival measurements were performed to investigate this phenomenon. A radio direction finder using the MUSIC algorithm [Schmidt, 1986], capable of discerning 8 incoming waves at the same time instant, was used to measure the spatial angle-of-arrival of the NVIS beacon 10 times per second.

These measurements showed that the beacon signal arrived via NVIS propagation during daytime, with all observed incoming waves arriving between $72^{\circ}$ and $80^{\circ}$ degrees elevation angle. At nighttime, when the Maximum Usable Frequency (MUF) had become too low to support NVIS, waves arriving at elevation angles above $60^{\circ}$ were no longer observed, indicating that a 'skip zone' was larger than $200 \mathrm{~km}$ had developed. The received signal, which was still $20 \mathrm{~dB}$ stronger than the background radio noise, arrived at elevation angles between $5^{\circ}$ and $45^{\circ}$, suggesting ionospheric propagation at low angles and backscatter at ground level, at distances between 1000 and $5000 \mathrm{~km}$. The measurements were repeated in a second experiment one month later with optimized transmitter parameters, to verify these initial findings and to measure both the spatial distribution of the incoming waves and of the (background) radio noise. Data analysis is in progress, to be followed by a publication. 


\subsubsection{Improved circular polarization measurement equipment}

In all circular polarization experiments in this thesis, the alternating circular polarization was obtained using phasing lines switched with coaxial relays, which limited the measurement speed to 2.5 seconds per sample and required precise tuning of the phasing lines with each frequency change. A new measurement set-up is planned using a dual channel coherent receiver [HPSDR] with a sampling speed meeting the Nyquist criterion, to obtain coherent samples on two perpendicular dipoles. From these samples, orthogonal linear and orthogonal circular polarizations can be calculated in the digital domain, which can be used to observe polarization with great time resolution or to realize very precise circular or elliptical polarization reception. Post-processing raw data allows simultaneous observations in linear and orthogonal circular or elliptical polarizations. The high measurement speed allows comparison of fading profiles on each of these antenna polarizations, even over shorter observation intervals (e.g. 10 minutes). The associated measurement set-up is depicted in Figure 10.1.

New beacon transmitters are realized using dual channel direct digital synthesis (DDS) to provide two synchronous outputs of which the phase difference and the amplitude is programmable and can be calibrated to compensate for phase errors in the amplifiers and low pass filters. The two transmitters outputs are connected to the dipoles of a Turnstile antenna, similar to the receive antenna.

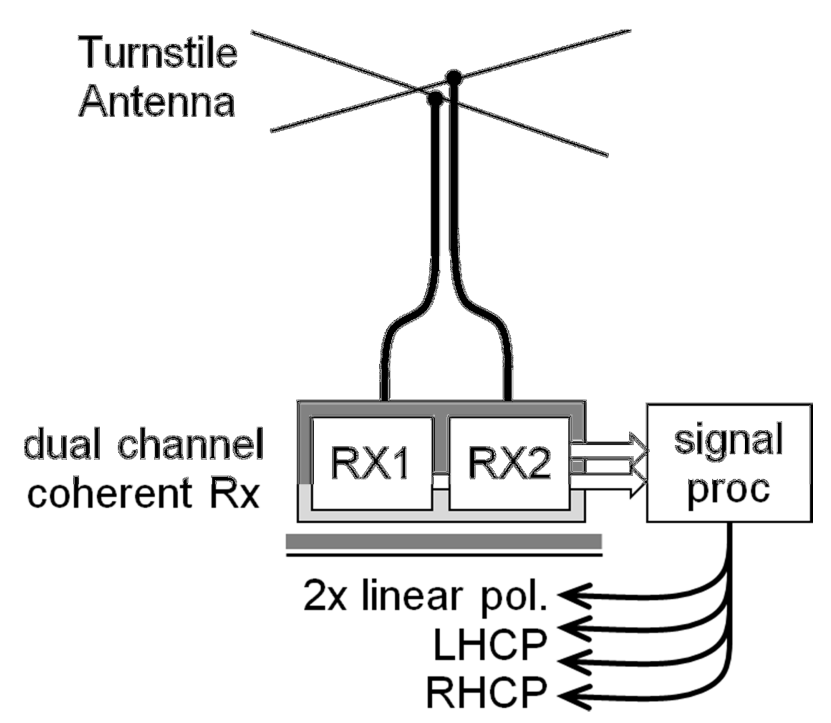

Figure 10.1 Experimental set-up for planned experiments, using a dual-channel coherent receiver to simultaneously monitor dual orthogonal linear and dual orthogonal circular polarizations. 


\subsubsection{Planned NVIS experiments in The Netherlands and Spain}

Using this new measurement set-up, an experiment is planned in The Netherlands (latitude $53^{\circ}$ North) and Spain (latitude $41^{\circ}$ North). Eight beacon transmitters will be placed at random azimuth and distance around the receiver and transmit alternatingly LHCP, RHCP and linear polarization. Goal of the experiment is to test the independence of the characteristic wave isolation of distance and azimuth and of geomagnetic dip angle. As the received polarization can be altered in post-processing, adaptation of the polarization to the actual propagation to achieve higher channel isolation can be studied. The measurements in Spain, for which sponsoring of the European Association on Antennas and Propagation (EurAAP) is obtained, will be performed in cooperation with LaSalle University Ramon Llull in Barcelona, Spain.

\subsection{NEW RESEARCH TOPICS}

The following research topics were identified that merit further research in the future:

\subsubsection{NVIS propagation}

More research is needed to identify the physical path structure that causes the characteristic multipath fading perceived in the NVIS channel, even when only one of the characteristic waves is excited, and only F2-layer propagation exists. This is necessary to understand the cause of this fading, which is necessary to devise measures to reduce this fading. Additional experiments on the nighttime above-the-MUF propagation over short-range paths are needed to clarify its nature.

\subsubsection{NVIS antennas}

Extension of the research on the influence of antenna height on NVIS antenna performance to other antenna types than the dipole antennas discussed in Sections 4.4 to 4.6 would be useful. NVIS Antenna Gain and NVIS Directivity simulations could be performed for a large number of antenna types, for several coverage area sizes, accompanied by their optimum dimensions and installation heights for several soil types. This data would provide an invaluable reference source for NVIS antenna selection.

Investigation into the use of arrays of active antennas could further improve the signal-tonoise ratio and at the same time reduce co-channel interference by the suppression of signals arriving at lower elevation angles in NVIS reception, as was indicated in paragraph 4.4.3. Experiments with adaptive polarization systems [Chu, 1971] to obtain the highest channel separation could increase the effectiveness of dual circular polarization systems regardless of the geomagnetic latitude and instantaneous propagation. 


\subsubsection{Radio noise}

More measurements that provide insight in the spatial distribution of ambient noise arriving via skywave, similar to those reported in [Posa, et al., 1972; Coleman, 2002], would provide valuable input to optimize this aspect of receive antennas. Similarly, measurements on the propagation of man-made noise from individual sources to the receiver, including skywave, would be helpful to establish a correct picture of the origin of its component and their spatial parameters.

More work is needed to realize radio noise measurement antennas that will produce true values regardless of polarization and spatial angle-of-arrival. If this could be combined with measurements of the spatial angle of arrival and the polarization of the radio noise, this would provide additional insight in radio noise that is now missing.

Adaptive filtering protocols to help discern radio noise from intended transmissions is needed to automate acquisition of the radio noise data that is needed for radio noise research that goes beyond the measurement of radio noise RMS values.

\subsubsection{NVIS channel simulation}

Recorded channel data from real NVIS transmissions under a large variety of propagation conditions would be invaluable to compare different protocols in realistic yet repeatable circumstances.

Studies into the conversion of recorded real-life propagation into a realistic channel simulator for F2-layer and E-layer NVIS propagation would be helpful to compare and improve modulation, coding and diversity protocols. These simulators should incorporate ordinary and extraordinary wave channels, both separately and in a combined fashion.

\subsubsection{Modulation systems for NVIS channels}

Modulation systems optimized for two orthogonal characteristic wave channels on the same frequency with minimum coding overhead have to be realized and tested.

Objective comparison of a large number of standardized HF communication protocols over identical - recorded - NVIS channels is desirable. Concerning HF radio communication protocols, two different lines of investigation are of equal interest: (a) protocols optimized to achieve the highest data rate in a $3 \mathrm{kHz}$ bandwidth, and (b) protocols that achieve highly reliable data transfer at low SNR's (below $6 \mathrm{~dB}$ ) in narrow bandwidths (e.g. $100 \mathrm{~Hz}$ ). The latter are invaluable for the transfer of lifesaving information when circumstances (antenna situation, battery power, propagation) are unfavorable. 


\section{References}

Adorian, P., and A. H. Dickinson (1952), "High Frequency Broadcast Transmission with Vertical Radiation," J. Br. IRE, pp. 111-116.

Ads, A., P. Bergadà, C. Vilella, J. Regué, J. Pijoan and R. Bardají (2012), "A Comprehensive Sounding of the lonospheric HF Radio Link from Antarctica to Spain," Radio Sci, 48, (1), pp. 1-12.

Ads, A. G. (2013), Soundings of the lonospheric HF Radio Link between Antarctica and Spain, PhD Thesis, Universitat Ramon Llull, Barcelona, Spain.

Appleton, E. V., and M. A. F. Bartlett (1925), "On Some Direct Evidence for Downward Atmospheric Reflection of Electric Rays," Proc. Royal Soc. London, 109, (752), pp. 621641.

Appleton, E. V., and G. Builder (1932), "Wireless Echoes of Short Delay," Proc. Phys. Soc., 44, (1), pp. 76-87.

Appleton, E. V., and G. Builder (1933), "The lonosphere as a Doubly-Refracting Medium," Proc. Phys. Soc., 45, (2), pp. 208-220.

Antoniou, S., L. Christofi, P. R. Green and G. F. Gott (2006), "High Rate Data Transmission in the Mid-Latitude NVIS HF Channel," IEE Proc. Comm., 153, (2), pp. 272-278.

Austin, B. A., and K. P. Murray (1988), "The Application of Characteristic-Mode Techniques to Vehicle-Mounted NVIS Antennas," IEEE Ant. Prop. Mag., 40, (1), pp. 7-30.

Austin, B. A., and K. P. Murray (1994), "Synthesis of Vehicular Antenna NVIS Radiation Patterns using the Method of Characteristic Modes," IEE Proc. Microw., Ant. Prop., 141, (4), pp. 151-154.

Austin, B. A. (2000), "Near Vertical Incidence Skywaves in World War II: An Historical Perspective," HF Radio Systems and Techniques, Guidford, UK, pp. 225-229.

Austin, B. A., and W. C. Liu (2002), "Assessment of Vehicle-Mounted Antennas for NVIS Applications," IEE Proc. Microw., Ant. Prop, 149, (3), pp. 147-152.

Austin, B. A. (2009), Optimum Antenna Height for Single-hop Oblique Incidence (NVIS) Propagation, http://www.ips.gov.au/IPSHosted/INAG/web-70/2009/optimum_anten na_height.pdf.

Ayeni, V., and G. Milward-Oliver (2005), Maitland+20: Fixing the Missing Link, Anima Centre, Bradford on Avon, UK.

Bandias, S., and S. R. Vemuri (2005), "Telecommunications Infrastructure Facilitating Sustainable Development of Rural and Remote Communities in Northern Australia", Telecommunications Policy, 29, 2, pp. 237-249. 
Barker, G. E., J. Taylor and G. H. Hagn (1971), Summary of Measurements and Modeling of the Radiation Patterns of Simple Field Antennas in Open (Level) Terrain, Mountains and Forests, Special Technical Report 45, U.S. Army Electronic Command, USA.

Barker, G. E. (1973), "Measurement of the Radiation Patterns of Full-Scale HF and VHF Antennas," Tr. Ant. Prop., 21, (4), pp. 538-544.

Beverage, H. H., and H. O. Peterson (1931), "Diversity Receiving System of R.C.A. Communications, Inc., for Radiotelegraphy", Proc. IRE, 19, (4), pp. 529-561.

Beverage, H. H. (1937), Frequency diversity, US Patent 2,067,432.

Bilitza, D., L. A. McKinnell, B. Reinisch and T. Fuller-Rowell (2011), "The International Reference Ionosphere (IRI) Today and in the Future," J. Geodesy, 85, (12), pp. 909-920.

Black, Q. R., J. F. Wood Jr. and W. M. Sherill (1995), "Mode Angles of Arrival in the 55- to 3500-km Range," Radio Sci, 30, (3), pp. 693-702.

Bodson, D. (1992), "When the Lines Go Down," IEEE Spectrum, 29, (3), pp. 40-44.

Breakall, J. K., J. S. Young, G. H. Hagn, R. W. Adler, D. L. Faust and D. H. Wemer (1994), "The Modeling and Measurement of HF Antenna Skywave Radiation Patterns in Irregular Terrain," IEEE Tr. Ant. Prop., 42, (7), pp. 936-945.

Brennan, D. G. (1961), "Probability Theory in Communication System Engineering," chapter in book Lectures on Communication System Theory, edited by E. Baghdady, McGrawHill, New York, USA.

Budden, K. G. (1985), The Propagation of Radio Waves, Cambridge University Press, UK.

Burgess, S. J., and N. E. Evans (1999), "Short-Haul Communications Using NVIS HF Radio", IEE Electr. Comm. Eng. J., 11, (2), pp. 95-104.

Burke, G. J., E. K. Miller, J. N. Brittingham, D. L. Lager, R. J. Lytle and J. T. Okada (1981), "Computer Modeling of Antennas Near the Ground," Electromagn., 1, (1), pp. 29-49.

Burke, G. J., E. K. Miller and A. I. Poggio (2004), "The Numerical Electromagnetics Code (NEC) - A Brief History," IEEE APS, Monterey, CA, USA.

Burke, G. J. (2011), Numerical Electromagnetics Code - NEC-4.2 Method of Moments, Part I: User's Manual, LLNL-SM-490875, Livermore, CA, USA.

Buys, P., S. Dasgupta and T. S. Thomas (2009), "Determinants of a Digital Divide in SubSaharan Africa: A Spatial Econometric Analysis of Cell Phone Coverage," World Development, 37, (9), pp. 1494-1505.

Cannon, P. S., M. J. Angling and N. G. Davies (2000), "DAMSON HF Channel Characterisation A Review," MILCOM, Los Angeles, CA, USA.

Cannon, P. S., M. J. Angling, J. A. T. Heaton and A. K. Shukla (2005), "The Effects of Space Weather on Radio Systems," in Effects of Space Weather on Technology Infrastructure, Springer Netherlands, pp. 185-201.

CCIR Report 65 (1957), Revision of Radio Noise Data, International Telecommunication Union, Geneva, Switzerland. 
CCIR Report 322-2 (1983), Characteristics and Applications of Atmospheric Radio Noise Data, International Telecommunication Union, Geneva, Switzerland.

CCIR Report 258-5 (1990), Man-Made Radio Noise, International Telecommunication Union, Geneva, Switzerland.

Chapman, S. (1939), "The Atmospheric Height Distribution of Band-Absorbed Solar Radiation," Proc. Phys. Soc., 51, 1, pp. 93-109.

Christofi, L. (2001), Measurement of HF NVIS Radio Channel Parameters with Application to the Design of Very High Rate Modems, PhD Thesis, University of Manchester, UK.

Chu, T.S. (1971), "Restoring the Orthogonality of Two Polarizations in Radio Communication Systems - I," Bell System Technical Journal, 50, 9, pp. 3063-3069.

Clarke, C. (1962), "Atmospheric Radio-Noise Studies based on Amplitude-Probability Measurements at Slough, England, during the International Geophysical Year," Proc. IEE B, 109, (47), pp. 393-404.

Coleman, C. J. (2002), "A Direction-Sensitive Model of Atmospheric Noise and its Application to the Analysis of HF Receiving Antennas," Radio Sci, 37, (3), pp. 3.1-3.10.

Comfort, L. K. (2006a), "Cities at Risk: Hurricane Katrina and the Drowning of New Orleans," Urban Affairs Review, 41, (4), pp. 501-516.

Comfort, L. K., and T. W. Haases (2006b), "Communication, Coherence and Collective Action: The Impact of Hurricane Katrina on Communications Infrastructure," Public Works Management \& Policy, 10, (4), pp. 328-343.

Cones, H. N., H. V. Cottony and J. M. Watts (1950), "A 600-Ohm Multiple-Wire Delta Antenna for lonosphere Studies," J. Res. NBS, 44, pp. 475-488.

Crichlow, W. Q., A. D. Spaulding, C. J. Roubique and R. T. Disney (1960), AmplitudeProbability Distributions for Atmospheric Radio Noise, NBS Monograph 23, Washington, USA.

Crichlow, W. Q., R. T. Disney and M. A. Jenkins (1966), Quarterly Radio Noise Data, June, July, August 1966, ESSA Techn. Rep. IER18-ITSA-18-31, ITSA, Boulder, Col., USA.

Cummings, N. P. (2005), "Design and Analysis of a Pattern Selectable Airborne HF Antenna," ACES, Honololu, Hawaii, pp. 499-502.

Davies, K. (1990), Ionospheric Radio, Peter Peregrinus Ltd., London, UK.

Davies, K., P. L. Cotterill, C. Ponsionen and V. Jodalen (2003), "On-Air Test and Evaluation of STANAG 4538," IRST, Bath, UK, pp. 13-18.

Davis, T. (2006), A Failure of Initiative: Final Report of the Select Bipartisan Committee to Investigate the Preparation for and Response to Hurricane Katrina, US Government Printing Office, Washington, DC, USA.

Dietrich, F. J., and R. K. Long (1969), "An Efficient Moderate-Size Vertical-Incidence Ionosonde Antenna for 2-20 MHz Polarization Studies," IEEE Tr. Ant. Prop., 17, (5), pp. 551-557. 
Digital Radio Mondiale (2009), DRM System Specification, ES 201980 V3.1.1, European Telecommunications Standards Institute, Sophia-Antipolis, France.

Dimension4, Thinkman software, http://www.thinkman.com/dimension4/default.htm.

Ellingson, S. (2005), "Antennas for the Next Generation of Low Frequency Radio Telescopes," IEEE Tr. Ant. Prop., 53, (8), pp. 2480-2489.

Essex, E. A. (1968), "Periodic Fading of Ionospheric Echoes," J. Atmosph. Terr. Phys., 30, (7), pp. 1441-1446.

Ewald, S., and R. Lindquist (2006), "The Katrina Chronicles 2," QST, 2006, (2), pp. 50-53.

Faraday, M. (1845), "On the Magnetization of Light and the Illumination of Magnetic Lines of Force," Philosophical Transactions.

Farmer, E. J. (1996), "NVIS Propagation at Low Solar Flux Indices," chapter in book Near Vertical Incidence Skywave Communication: Theory, Techniques and Validation, edited by D. M. Fiedler and E. J. Farmer, Worldradio Books, Sacramento, USA.

Fiedler, D. M., and E. J. Farmer (1996), Near Vertical Incidence Skywave Communications, Theory, Techniques and Validation, Sacramento, Worldradio Books.

Friis, H. T., and C. B. Feldman (1937), "A Multiple-Unit Steerable Antenna for Short-Wave Reception," Proc. IRE, 25, (7), pp. 841-917.

Furman, W. N., and E. Koski (2009), "Standarization of an Intermediate Duration HF Channel Variation Model," IRST, Edinburgh, UK, pp. 137-141.

Furman, W. N., and J. W. Nieto (2012), "Latest On-Air Testing of U.S. MIL-STD-188-110C Appendix D Wideband HF Data Waveforms," IRST, Edinburgh, UK, pp. 1-5.

Friis, H. T., and C. B. Feldman (1937), "A Multiple Unit Steerable Antenna for Short-Wave Reception", Proc. IRE, 25, (7), pp. 841-917.

Gerritsen, H. (2005), "What Happened in 1953? The Big Flood in the Netherlands in Retrospect," Phi. Tr. Royal Soc. A, 363, (1831), pp. 1271-1291.

Gibson, A. J., and P. A. Bradley (1991), "A New Formulation for Above-the-MUF Loss," IRST, Edinburgh, UK.

Gil, U., D. Guerra, I. Pena, D. de la Vega, P. Angueira, M. Vélez and G. Prieto (2012), "Analysis of the NVIS Channel Availability in the Medium Wave Band," BMSB, Seoul, Korea, pp. 1-6.

Gil, U., J. A. Garcia, J. L. Garcia, J. Sanchez, D. Guerra, I. Pena and D. de la Vega (2013), "Near Vertical Incidence Skywave Field Strength Time Variability Characterization in the Medium Wave Band," EuCAP, Gothenburg, Sweden, pp. 2615-2618.

Goodman, J. M. (2005), "Operational Communication Systems and Relationships to the Ionosphere and Space Weather," Advances in Space Research, 36, pp. 2241-2252.

Guerra, D., M. A. Coleto, L. Melgar, U. Gil, I. Pena, G. Prieto and I. Angulo (2013), "Measurement Based Near Vertical Incidence Skywave Channel Model in the Medium Wave Band," EuCAP, Gothenburg, Sweden, pp. 3403-3407. 
Gunashekar, S. D., E. M. Warrington, S. D. Gunashekar, S. Salous, S.M. Feeney, H. Zhang, N. Abbasi, L. Bertel, D. Lemur and M. Oger (2009), "Investigation into the Feasibility of MIMO Techniques within the HF Band - Preliminary Results", Radio Sci, 44, (1).

Hagn, G. H., and J. E. Van der Laan (1970), "Measured Relative Responses Toward the Zenith of Short-Whip Antennas on Vehicles at High Frequency," IEEE Tr. Veh. Techn., 19, (3), pp. 230-236.

Hagn, G. H. (1973), "On the Relative Response and Absolute Gain toward the Zenith of HF Field-Expedient Antennas - Measured with an Ionospheric Sounder," IEEE Tr. Ant. Prop., 21, (4), pp. 571-574.

Hathaway, D. H., Solar Cycle Prediction, NASA, MSFC, http://solarscience.msfc.nasa.gov/ predict.shtml.

Heising, R. A. (1940), "Radio Extension Links to the Telephone System," Bell System Technical Journal, 19, (4), pp. 611-646.

Hervás, M., J. L. Pijoan, R. Alsina-Pagès, M. Salvador and D. Altadill (2013), "Channel Sounding and Polarization Diversity for the NVIS Channel," Nordic HF, Fårö, Sweden.

Horner, F. (1953), "The Reception of Atmospherics at High Frequencies," J. Atm. Terr. Phys., 4, 3, pp. 129-140.

Hoult, N. S., J. R. Whiffen, M. H. Tooby and P. C. Arthur (2000), "16 kbps Modems for the HF NVIS Channel," HFRST, Guildford, UK, pp. 317-321.

HPSDR, High Performance Software Defined Radio, www.openhpsdr.org.

Hunsucker, R. D., and J. K. Hargreaves (2002), The High-Latitude lonosphere and its Effects on Radio Propagation, Cambridge University Press, London, UK.

ITU Handbook on Emergency Telecommunications (2005), International Telecommunications Union, Geneva, Switzerland.

ITU-R Rec. F.520-2 (1992), Use of High Frequency Ionospheric Simulators, International Telecommunications Union, Geneva, Switzerland.

ITU-R Rec. F.1487-0 (2000), Testing of HF Modems with Bandwidths of up to about $12 \mathrm{kHz}$ using Ionospheric Channel Simulators, International Telecommunications Union, Geneva, Switzerland.

ITU-R Report P.266-7 (1990), lonospheric Propagation and Noise Characteristics Pertinent to Terrestrial Radiocommunication Systems Design and Service Planning, International Telecommunications Union, Geneva, Switzerland.

ITU-R Rec. P.372-6 (1994), Radio Noise, International Telecommunication Union, Geneva, Switzerland.

ITU-R Rec. P.372-10 (2009), Radio Noise, International Telecommunication Union, Geneva, Switzerland.

ITU-R Rec. P.372-11 (2013), Radio Noise, International Telecommunication Union, Geneva, Switzerland. 
ITU-R Rec. P.434-6 (1995), Reference lonospheric Characteristics and Methods of Basic MUF, Operational MUF and Ray-Path Prediction, International Telecommunications Union, Geneva, Switzerland.

ITU-R Rec. P.1407-5 (2013), Multipath Propagation and Parameterization of its Characteristics, International Telecommunication Union, Geneva, Switzerland.

ITU-R Report P.2011-1 (1997), Propagation at Frequencies above the Basic MUF, International Telecommunications Union, Geneva, Switzerland.

ITU-R Report SM.2055 (2006), Radio Noise Measurements, International Telecommunication Union, Geneva, Switzerland.

Jansky, K. G. (1932), "Directional Studies of Atmospherics at High Frequencies," Proc. IRE, 20, (12), pp. 1920-1932.

Jasik, H. (1961), Antenna Engineering Handbook, McGraw-Hill, pp. 21-26.

Jenkins, R. W. (1995), Antenna Amplitude and Phase Pattern Measurements using an Aircraft-Towed Transmitter, CRC Report 95-003, Communications Research Centre, Ottawa, Canada.

Jodalen, V., B. Lundborg and B. Jacobsen (2000), "Channel Characteristics of HF NVIS Paths in Northern Scandinavia," HFRST, Guildford, UK, pp. 269-273.

Jodalen, V., T. Bergsvik, P. Cannon and P. C. Arthur (2001), "Performance of HF Modems on High-Latitude Paths Using Multiple Frequencies," Radio Sci, 36, (6), pp. 1687-1698.

Johnson, R. C. (1993), Antenna Engineering Handbook, McGraw-Hill, 3rd ed., New York, USA.

Johnson, E. E. (2006), "HF Radio Mesh Networks," MILCOM, Washington, DC, USA.

Johnson, E. E. (2007), "NVIS Communications during the Solar Minimum," MILCOM, Orlando, FLA, USA.

Jones, T. O. (2014), "Directional Array for Near-Vertical-Incidence Skywave (NVIS)," IEEE Ant. Prop. Mag., 56, (2), pp. 132-143.

Kaiser, M. L. (1993), "Time-Variable Magnetospheric Radio Emissions from Jupiter," J. Geophys. Res.: Planets, 98, (E10), pp. 18757-18765.

Kennelly, A. E. (1902), "On the elevation of electrically-conducting strata of the earth's atmosphere," Elec. World \& Eng., 39, (11), p. 473.

Knudsen, O. (1976), "The Faraday Effect and Physical Theory 1845-1873," Archive for History of Exact Sciences, 5, (3), pp. 235-281.

Kobayashi, M. (2014), "Experience of Infrastructure Damage Caused by the Great East Japan Earthquake and Countermeasures against Future Disasters," IEEE Comm. Mag., 52, (3), pp. 23-29.

Kraus, J. D. (1988), Antennas, 2nd Edition, McGraw-Hill, New York, USA.

Krause, M. (2013), Calibration of the LOFAR Antennas, Master Thesis, University of Nijmegen, The Netherlands. 
Kwasinski A., W. W. Weaver, P. T. Krein and P. L. Chapman (2006), Hurricane Katrina: Damage Assessment of Power Infrastructure for Distribution, Telecommunication, and Backup, Technical Report CEME-TR-06-05, University of Illinois, USA.

Kwasinski, A, W. W. Weaver, P. L. Chapman and P. T. Krein (2009), "Telecommunications power plant damage assessment for Hurricane Katrina-site survey and follow-up results," IEEE Systems Journal, 3, (3), pp. 277-287.

Kwasinski, A. (2011a), "Effects of Notable Natural Disasters from 2005 to 2011 on Telecommunications Infrastructure: Lessons from On-site Damage Assessments," INTELEC, Amsterdam, The Netherlands.

Kwasinski, A., and A. K. Tang (2011b), "Telecommunications Performance in the $M=9.0$ OffShore East Coast of Japan Earthquake and Tsunami," ISELLGE, Tokyo, Japan.

Lamy-Bergot, C., S. Herry, J. Y. Bernier and F. Ngo Bui Hun (2013), "On-Air Tests Results for HF XL Wideband Modem," Nordic HF, Fårö, Sweden.

Leferink, F., F. Silva, J. Catrysse, S. Batterman, V. Beauvois and A. Roc'h (2010), "Man-Made Noise in Our Living Environments," Radio Science Bulletin, 2010, (334), pp. 49-57.

Lefeuvre, F., and T. J. Tanzi (2014), "Radio Science's Contribution to Disaster Emergencies," Radio Science Bulletin, 348.

Le Masson, J, Y. M. Erhel and A. R. Mamane, "Simulated Performance of the STANAG 4538 HF Transmission Standard," IRST, York, UK.

Linden, L. F. (2004), "Winlink 2000 in the Jungle," QST, 2004 (11), pp. 42-45.

Lindquist, R. (2005), "The Katrina Chronicles," QST, 2005, (11), pp. 43-48.

Losada, I. (2009), DRM MW NVIS Nighttime Quality Assessment and Channel Analysis, PhD Thesis, University of the Basque Country, Bilbao, Spain.

Lossmann, E., M.-A. Meister and U. Madar (2011), "On HF Communication Link Parameter Estimation in the Baltic Region," APWC, Torino, Italy, pp. 812-814.

Lowell Digisonde International, www.digisonde.com.

Magdaleno, S., D. Altadill, M. Herraiz, E. Blanch and B. de la Morena (2011), "lonospheric Peak Height Behavior for Low, Middle and High Latitudes: A Potential Empirical Model for Quiet Conditions - Comparison with the IRI-2007 model," J. of Atm. and Solar-Terr. Phys., 73, (13), pp. 1810-1817.

Maitland, D. (1984), The Missing Link - Report of the International Commission for WorldWide Telecommunications Development, International Telecommunication Union, Geneva, Switzerland.

Martínez, A, V. Villarroel, J. Seoane and F. Del Pozo (2004), "Rural Telemedicine for Primary Healthcare in Developing Countries," IEEE Technology and Society Magazine," 23, 2, pp. 13-22.

Martyn, D. F., R. O. Cherry and A. L. Green (1935), "Long Distance Observations of Radio Waves of Medium Frequencies," Proc. Phys. Soc., 47, (2), pp. 340-351. 
Maunsell, H. I., and J. W. Stafford (1964), "Telstar Design and Construction and Early Results of the Scientific Space Experiments," IEEE Tr. on Comm. and Electr., 83, (70), pp. 27-37.

Maxwell, J. C. (1873), "A Treatise on Electricity and Magnetism, Part II," Clarendon Press, Oxford, UK.

McNamara, L. F. (1991), The Ionosphere: Communications, Surveillance and Direction Finding, Krieger Publishing Company, Malabar, FLA, USA.

McNamara, L. F., T. W. Bullett, E. Mishin and Y. M. Yampolski (2008), "Nighttime Above-theMUF HF Propagation on a Midlatitude Circuit," Radio Science, 43, 2, pp. 1-8.

McNicol, R. W. E. (1949), "The Fading of Radio Waves of Medium and High Frequencies," Proc. IEE III, 96, (44), pp. 517-524.

McSwain, N. E. (2010), "Disaster Response. Natural Disaster: Katrina," Surgery Today, 40, pp. 587-591.

Mervin, K. J., G. Radley, G. W. Gilman and R. J. Halsey (1955), "A Transatlantic Telephone Cable," Bell System Technical Journal, 102, (2), pp. 117-130.

Mikami, T., T. Shibayama and S. Esteban (2012), "Field Survey of the 2011 Tohuku Earthquake and Tsunami in Miyagi and Fukushima Prefectures," Coastal Eng. Journal, 54, (1), pp. 117-130.

Miller, R. (2006), Hurricane Katrina: Communications \& Infrastructure Impacts, Nat. Defense Univ., Fort McNair, DC, USA.

Milward-Oliver, G. (2005), Maitland+20: Fixing the Missing Link, Anima Centre, Bradford on Avon, UK.

Nakagami, M. (1960), The m-Distribution, a General Formula of Intensity of Rapid Fading, Statistical Methods in Radio Wave Propagation, Pergamon Press, Oxford, UK.

NATO (1989), Characteristics of 1200/2400/3600 Bits per Second Single Tone Modulators/ Demodulators for HF Radio Links, STANAG 4285, NATO Standardization Organization, Brussels, Belgium.

NATO (1999), Characteristics of a Robust, Non-Hopping, Serial Tone Modulator/Demodulator for Severely Degraded HF Radio Links, STANAG 4415, NATO Standardization Organization, Brussels, Belgium.

NATO (2009), Technical Standards for an Automatic Radio Control System (ARCS) for HF Communication Links, STANAG 4538, NATO Standardization Organization, Brussels, Belgium.

Ndao, P. M., D. Lemur, Y. Erhel and C. Brousseau (2009), "Capacity Estimation of MIMO lonospheric Channels," IRST, Edinburgh, UK.

Ndao, P. M., Y. Erhel, D. Lemur, M. Oger and J. Le Masson (2013), "Development and Test of a Trans-Horizon Communication System Based on a MIMO Architecture," Eurasip J. Wireless Comm. Netw., 2013, (1), pp. 1-13. 
Ossakow, S. L. (1981), "Spread-F Theories - A Review," J. Atm. Terr. Phys., 43, (5/6), pp. 437452.

Papoulis, A., and S. U. Pillai (2002), Probability, Random Variables and Stochastic Processes, 4th edition, McGraw-Hill, New York, USA.

Pawsey, J. L., L. L. McCready and F. F. Gardner (1951), "Ionospheric Thermal Radiation at Radio Frequencies," J. Atm. Terr. Phys., 1, (5-6), pp. 261-277.

Pérez-Álvarez, I., S. Zazo, M. Ghogho, J. López-Pérez, B. Pérez-Díaz and E. Jiménez-Yguacel (2012), "Experimental Results on Multicarrier MIMO HF Communications," IRST, York, UK.

Perkiomaki, J. (2003-2014), HF Propagation Prediction and lonospheric Communications Analysis, www.voacap.com.

Perlman, S. (1970), High Frequency SAFOC Digital Data Link Tests, R\&D Techn. Rep. ECOM3341, ECOM US Army Electronics Command, Ft. Monmouth, NJ, USA

Physikalisch-Technische Bundesanstalt, Braunschweig, Germany, http://www.ptb.de/cms/ en/fachabteilungen/abt4/fb-44/ag-442/dissemination-of-legal-time/dcf77.html.

Pijoan, J. L., D. Altadill, J. M. Torta, R. M. Alsina-Pagès, S. Marsal and D. Badia (2014), "Remote Geophysical Observatory in Antarctica with HF Data Transmission: A Review," Remote Sens., 6, pp. 7233-7259.

Polycarpou, A. C., and C. A. Balanis (2000), "Rotor Modulation of Helicopter Antenna Characteristics," APS, Salt Lake City, UT, USA.

Posa, L. M., D. J. Materazzi and C. Gerson (1972), "Azimuthal Variation of Measured HF Noise," IEEE Tr. EMC, 14, (1), pp. 21-31.

Potter, R. K. (1932), "High-Frequency Atmospheric Noise," Proc. IRE, 19, (10), pp. 1731-1765.

Proakis, J. G. (1995), Digital Communications, 3rd edition, McGraw-Hill, New York, USA.

Ratcliffe, J. A. (1962), The Magneo-Ionic Theory and its Application to the lonosphere, Cambridge University Press, London, UK.

Rawer, K. (1993), Wave propagation in the lonosphere, Kluwer Academic, Dordrecht, The Netherlands.

Ray, W. A., G. E. Barker and S. S. Martensen (1966), Full-Scale Pattern Measurements of Simple HF Field Antennas in a US Conifer Forest, Spec. Tech. Rep. 25, Stanford Research Inst., Menlo Park, CA, USA.

Reber, G. (1944), "Cosmic Static," Astrophysical Journal, 1944, (100), pp. 279-287.

Reilly, M. H. (1991), "Upgrades for the Efficient Three-Dimensional lonospheric Ray Tracing: Investigation of HF Near Vertical Incidence Sky Wave Effects," Radio Sci, 26, (4), pp. 971-980.

Reilly, M.H. (2000), "Ray Trace Calculation of Ionospheric Propagation at Lower Frequencies," Radio Sci, 41, (5). 
Richie, J. E., and T. Joda (2003), "HF Antennas for NVIS Applications Mounted to Helicopters with Tandem Main Rotor Blades," IEEE Tr. EMC, 45, (2), pp. 444-448.

Rohde \& Schwarz (2009), R\&S FSMR Measuring Receiver, Data Sheet Version 7.00, München, Germany.

Rollema, D. (2004), "Amateur Radio Emergency Network During 1953 Flood," Proc. IEEE, 94, (4), pp. 759-762.

Royal Observatory of Belgium, http://digisonde.oma.be/.

Rufenach, C. L., and G. H. Hagn (1966), Comparison of C-2 lonospheric Sounder Data with Frequency Predictions for Short-Range Communication with Man-Pack Transceivers in Thailand, Spec. Techn. Rep. 15, Stanford Research Institute, Menlo Park, CA, USA.

Runge, W. (1932), Polarization Diversity Reception, US Patent 1,892,221.

Rush, C. M., and J. Buchau (1977), "Determining the F-Region Critical Frequency from Satellite-Borne Noise Measurements," J. Atm. Terr. Phys., 39, pp. 277-286.

Ryle, M. (1948), "Some Observations of Solar Radiation on Wavelengths of 1.7 and 3.8 Metres," Conv. Sc. Radio, pp. 333-334.

Saakian, A. (2012), "CEM Optimization of the HF Antennas Installations Onboard the Aircraft," IEEE APS, Chicago, USA.

Sanz-González, J. L., S. Zazo-Bella, I. A. Perez-Alvarez and J. Lopez-Perez (2009), "Parameter Estimation Algorithms for Ionospheric Channels," IRST, Edinburgh, UK.

Schmidt, R. O. (1986), "Multiple Emitter Location and Signal Parameter Estimation," IEEE Tr. Ant. Prop., 34, (3), pp. 276-280.

SCS - Spezielle Communications Systeme GmbH \& Co. KG, Hanau, Germany, www.p4dragon. com/en/PACTOR-4.html.

Secan, J. A., and P. J. Wilkinson (1997), "Statistical Studies of an Effective Sunspot Number," Radio Sci, 32, (4), pp. 1717-1724.

Simons, J. M. M. (2006), "Effective Directivity for Shortwave Reception by DSP," QEX, 2006, (7).

Sims, B. (2007), "The Day After the Hurricane: Infrastructure, Order, and the New Orleans Police Department's Response to Hurricane Katrina," Social Studies of Science, 37, (1), pp.111-118.

Sklar, B. (1997), "Rayleigh Fading Channels in Mobile Digital Communication Systems Part II: Mitigation," IEEE Comm. Mag., 35, (9), pp. 148-155.

Smith, N. (1939), "The Relation of Radio Skywave Transmission to Ionosphere Measurements," Proc. IRE, 27, (5), pp. 332-347.

Solar Terrestrial Dispatch (2001), Internet Space Weather and Radio Propagation Forecasting Course, Stirling, Alberta, Canada.

Solar Terrestrial Dispatch (2010), Proplab-Pro version 3, Stirling, Alberta, Canada. 
Spaulding, A. D. and R. T. Disney (1974), Man-Made Radio Noise - Part I: Estimates for Business, Residential, and Rural Areas, OT Report 74-38, USGPO, Washington, DC, USA.

Stankov, S. M., J. C. Jodogne, I. Kutiev, K. Stegen and R. Warnant (2012), "Evaluation of Automatic Ionogram Scaling for use in Real-Time Ionospheric Density Profile Specification: Dourbes DGS-256/ARTIST-4 Performance," Annals of Geophysics, 55, (2), pp. 283-291.

Strangeways, H. J. (2006), "Estimation of Signal Correlation at Spaced Antennas for MultiModed lonospherically Reflected Signals and its Effect on the Capacity of SIMO and MIMO HF Links," IRST, London, UK, pp. 306-310.

Straw, R. D. (2005), "What's the Deal about NVIS?," QST, 2005, (12), pp. 38-43.

Tang, A., D. C. Ray, D. Ames, C. V. R. Murty, S. K. Jain, S. R. Dash, H. B. Kaushik, G. Mondal, G. Murugesh, G. Plant, J. McLaughlin, M. Yashinsky, M. Eskijian and R. Surrampallih (2004), "Lifeline Systems in the Andaman and Nicobar Islands (India) after the December 2004 Great Sumatra Earthquake and Indian Ocean Tsunami," Earthquake Spectra, 22, (S3), pp. 581-606.

Taylor, J. H., Weak Signal Propagation Reporter, http://physics.princeton.edu/pulsar/K1JT/ wspr.html.

Thomas, H. A. (1950), "A Subjective Method of Measuring Radio Noise," Proc. IEE III, 97, (49), pp. 329-334.

Tooby, M. H., P. C. Arthur and P. L. Cotterill (1999), "Investigations into the Channel Characteristics of HF NVIS Links and the Implications for High Data Rate Transfer," IEE Coll. on Freq. Sel. and Mgt. Techn. for HF Comm., London, UK.

Tooby, M. H., P. C. Arthur, P. L. Cotterill, N. S. Hoult and J. R. Whiffen (2000), "An Assessment on the Propagation Characteristics of the NVIS Channel," HFRST, pp. 263-267.

US Department of Defense (2011), Interoperability and Performance Standards for Data Modems, MIL-STD-188-110C, Department of Defense Standardization Program, Philadelphia, PA, USA.

Van Maanen, E. (2006), "Practical Radio Noise Measurements," Int. Symp. on EMC, Wroclaw, Poland, pp. 427-432.

Van Veen, B. D., and K. M. Buckley (1988), "Beamforming: A Versatile Approach to Spatial Filtering," IEEE Acoustics, Speech and Signal Proc. Mag., 5, (2), pp. 4-24.

Vilella, C., D. Miralles and J. Pijoan (2008), "An Antarctica-to-Spain HF lonospheric Radio Link: Sounding Results," Radio Sci, 43, (4).

Vlasic, R., and D. Sumic (2008), "An Optimized Shipboard HF Loop Antenna for NVIS Link," ELMAR, Zadar, Croatia.

Voors, A. (2012), NEC-based Antenna Modeler and Optimizer, 4Nec2 version 5.8.12, http://www.qsl.net/4nec2/. 
Wagner, L. S., and J. A. Goldstein (1995), Channel Spread Parameters for the High-Latitude, Near-Vertical-Incidence-Skywave HF Channel: Correlation with Geomagnetic Activity, NRL/FR/5550-95-9772, Naval Research Lab., Washington, DC, USA.

Walden, M. C. (2009), "The Extraordinary Wave Mode: Neglected in Current Practical Literature for HF NVIS Communications," IRST, Edinburgh, UK.

Walden, M. C. (2012), "Comparison of Propagation Predictions and Measurements for MidLatitude HF Near-Vertical Incidence Sky Wave links at 5 MHz," Radio Sci, 47, (4).

Wallace, M. A. (1992), "HF Radio in Southwest Asia," IEEE Comm. Mag., 30, (1), pp. 58-61.

Warrington, E. M., C. A. Jackson and B. Lundborg (2000), "Directional Diversity of HF Signals Received over High Latitude Paths, and the Possibility of Improved Data Throughput by Means of Spatial Filtering," IEE Proc. Microw. Ant. Prop., 147, (6), pp. 487-494.

Warrington, E. M., A. J. Stoker and D. R. Siddle (2006), "Measurement and Modeling of HF Channel Directional Spread Characteristics for Northerly Paths," Radio Sci, 41, (2).

Watson Watt, R. A., and E. V. Appleton (1938), "On the Nature of Atmospherics - Part I," Proc. R. Soc. A, 103, pp. 84-102.

Watterson, C. C., J. R. Juroshek and W. D. Bensema (1970), "Experimental Confirmation of an HF Channel Model," IEEE Tr. Comm. Techn., 18, (6), pp.792-803.

Webber, W. (1962), "The Production of Free Electrons in the Ionospheric D Layer by Solar and Galactic Cosmic Rays and the Resultant Absorption of Radio Waves," J. Geophys. Res., 67, (13), pp. 5091-5106.

Wheeler, J. L. (1966), "Transmission Loss for Ionospheric Propagation above the Standard MUF," in Radio Sci, 1, 11, pp. 1303-1308.

Wheelon, A. D. (1952),"Refractive Corrections to Scatter Propagation," J. Geophys. Res., 62, (3), pp. 343-349.

Wilkinson, P. J. (2004), "Ionospheric Variability and the International Reference lonosphere", Adv. Space Res., 34, (9), pp. 1853-1859.

Witte, A. (2008), A Broadband Antenna for lonospheric Sounding, MSc project, Royal Institute of Technology, Stockholm, Sweden.

Witvliet, B. A., E. van Maanen, A. J. Westenberg and G. Visser (2005), "Elevation Angle Measurements for NVIS Propagation," RadCom, 81, (6), pp. 76-79.

Witvliet, B. A., E. van Maanen, G. J. Petersen, A. J. Westenberg, M. J. Bentum, C. H. Slump and R. Schiphorst (2014), "The Importance of Circular Polarization for Diversity Reception and MIMO in NVIS Propagation," EUCAP, The Hague, The Netherlands.

Witvliet, B. A., E. van Maanen, G. J. Petersen, A. J. Westenberg, M. J. Bentum, C. H. Slump and R. Schiphorst (2015a), "Near Vertical Incidence Skywave Propagation: Elevation Angles and Optimum Antenna Height for Horizontal Dipole Antennas," IEEE Ant. Prop. Mag., 57, (1), pp. 129-146. 
Witvliet, B. A., E. Van Maanen, G. J. Petersen, A. J. Westenberg, M. J. Bentum, C. H. Slump and R. Schiphorst (2015b), "Characteristic Wave Diversity in Near Vertical Incidence Skywave Propagation," EUCAP, Lisbon, Portugal.

Witvliet, B. A., E. Van Maanen, G. J. Petersen, A. J. Westenberg, M. J. Bentum, C. H. Slump and R. Schiphorst (2015c), "Measuring the Isolation of the Circularly Polarized Characteristic Waves in NVIS Propagation," IEEE Ant. Prop. Mag., 57, (3), pp. 120-130, 145.

Zolesi, B., and L. R. Cander (2014), lonospheric Prediction and Forecasting, Springer, Berlin, Germany. 
- 170 - 


\section{Abbreviations}

\begin{tabular}{|c|c|}
\hline ALE & Automatic Link Establishment \\
\hline AM & Amplitude Modulation \\
\hline ARQ & Automatic Repeat Query \\
\hline AUT & Antenna Under Test \\
\hline CCIR & Comité Consultatif International pour la Radio \\
\hline $\mathrm{CP}$ & Circular Polarization \\
\hline DAMSON & Doppler And Multipath SOunding Network \\
\hline DANL & Displayed Average Noise Level \\
\hline DDS & Direct Digital Synthesis \\
\hline Dimension4 & Time synchronization software using internet time servers \\
\hline DRM & Digital Radio Mondiale \\
\hline ECC & Emergency Communications Center \\
\hline EurAAP & European Association on Antennas and Propagation \\
\hline D-layer & ionospheric layer at approx. $60-90 \mathrm{~km}$ altitude \\
\hline $\mathrm{dBi}$ & antenna gain relative to an isotropic radiator in $\mathrm{dB}$ \\
\hline dBW & power in $\mathrm{dB}$ relative to $1 \mathrm{Watt}$ \\
\hline DCF77 & German time signal transmitter at $77.5 \mathrm{kHz}$ \\
\hline DF & Discrimination Factor \\
\hline E-layer & ionospheric layer at approx. 90-150 km altitude (Kennelly-Heaviside layer) \\
\hline EMC & ElectroMagnetic Compatibility \\
\hline Es & sporadic E-layer patches of high ionization \\
\hline F-layer & ionospheric layer at approx. 150-500 km altitude (Appleton-Barnett layer) \\
\hline FM & (required) Fading Margin \\
\hline foE & critical frequency of the E-layer of the ionosphere, for the ordinary wave \\
\hline foF2 & critical frequency of the F2-layer of the ionosphere, for the ordinary wave \\
\hline fxF2 & critical frequency of the F2-layer of the ionosphere, for the extraordinary wave \\
\hline $\mathrm{fv}$ & equivalent vertical frequency of the ionosphere \\
\hline
\end{tabular}




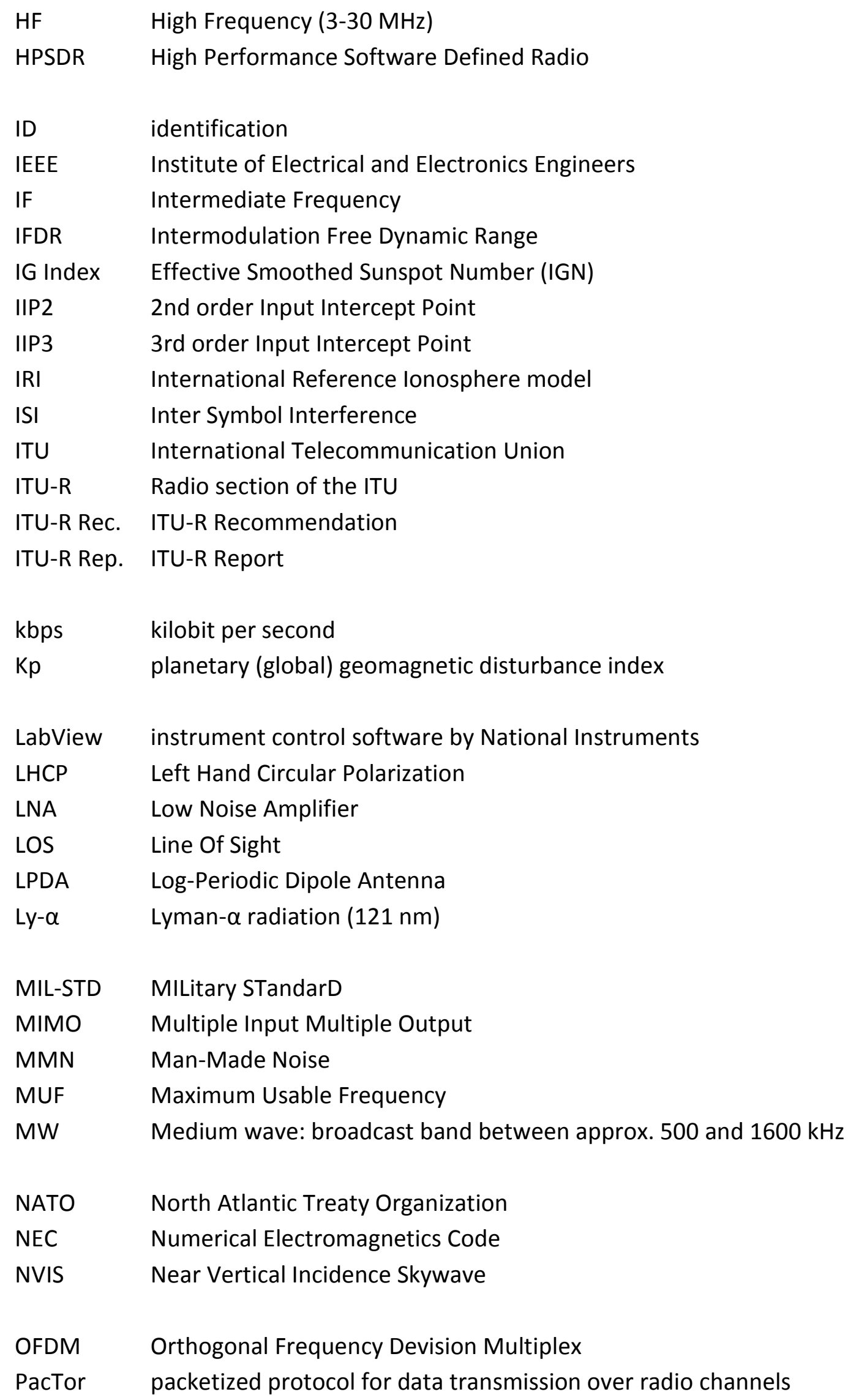


RDF Radio Direction Finder

RHCP Right Hand Circular Polarization

RF Radio Frequency

RMS Root Mean Square

Rx receiver

SDR Software Defined Radio

SISO Single Input Single Output

SLVM Short Lossless Vertical Monopole

SNR Signal-to-Noise Ratio

SSN Smoothed Sunspot Number

STANAG NATO STANdardization AGreement

TDL Tapped Delay Line

Tx transmitter

UHF Ultra High Frequency (300 MHz - $3 \mathrm{GHz}$ )

UK United Kingdom

UN United Nations

USA United States of America

UTC Coordinated Universal Time

UV Ultra Violet radiation $(10-420 \mathrm{~nm})$

VHF Very High Frequency $(30-300 \mathrm{MHz})$

VOACAP propagation prediction program, created by the Voice of America (VOA) HF broadcasting organization

WSPR Weak Signal Propagation Reporter transmission protocol

X-ray Röntgen radiation (1 pm - $10 \mathrm{~nm}$ )

4 -FSK 4 Frequency Shift Keying

4Nec2 NEC-based antenna simulation software 
- 174 - 


\section{Acknowledgment}

The author would like to thank all those who supported this promotion research:

Rolanda van der Helm, my girlfriend and soulmate, who made the disappointments light, the time pressure bearable, and shared the successes. She understood my enthusiasm and drive, accepted the extreme time scarcity caused by the promotion research and put up with me when I was tired and irritable, always there when I needed her. Thank you, Rolanda.

Mr. Peter Spijkerman, general director of Radiocommunications Agency Netherlands, for supporting my part-time promotion research. Also for the use of the professional measurement equipment of the Radiocommunications Agency, indispensible for precise measurements and equipment calibration.

Dr. Mark Bentum, prof. Kees Slump and dr. Roel Schiphorst, my Promotors, who tried to teach me academic writing and thinking, and at least partially succeeded. For reviewing all my drafts, revised and final publications, always coming up with very good improvements even under unpleasant deadlines. I admire your enthusiasm, professionalism and patience. Thanks for all the discussions and support.

Erik van Maanen, George Petersen and Albert Westenberg, three colleagues and radio hobbyists. Without their enthusiastic and enduring help none of the NVIS experiments would have been possible. I would call to ask: "I have an interesting experiment that no one has done before, but it requires a lot of preparation, followed by high precision field measurements that last several days and nights. Would you like to participate?"

Which translates to: "It will be cold, difficult, you will get no sleep and I expect very high performance of you. No payment." On which they would reply: "Yes, of course!" I know they enjoyed the adventure, and so did I.

Dr. Helmut Leonhard, who's discussions blended ideas, visions and technical reality, and sparked the wish to explore farther horizons. His contact with the University also laid the basis for this promotion research. Criticism of my colleagues that I start to sound like Helmut is a compliment. But it doesn't make sense: I don't have that accent.

Prof. Linda Doyle of Trinity College, University of Dublin, for making me understand that promotion research is not aiming at the Nobel prize, as part-time PhD students often seem to think. Switching back to second gear made PhD research fun again, and avoided a burnout. Thanks Linda, that one long talk in Dublin proved very valuable to me! 
Hans Houdijk, Ronald Agema, John Derksen and Ben van Duijvenvoorde, in their role of formal contacts of the Radio Agency in the promotion research. Each of them was very interested in my research, guarded my well-being and enjoyed my successes. Very much appreciated!

Dr. Sjoert Fleurke of Radiocommunications Agency Netherlands, for practical advice and encouragement, especially at the start, when the academic processes were still strange and new.

Bill Liles of Reston, Virginia, USA, who contacted me at regular intervals with new NVIS articles to read, with encouraging reactions to my work, good discussions and information about other groups doing related research. Thanks Bill!

Goos Visser of Radiocommunications Agency Netherlands for assistance with accurate phase delay measurements and technical discussions.

Dr. Rosa Ma Alsina-Pagès of LaSalle University Ramon Lllull, Barcelona, for the excellent cooperation while writing the NVIS Overview article. Hope to work together on more research in the future!

Mr. and Mrs. Overbeek for the use of their property in Ambt Delden for the circular polarization measurements. They define the word 'hospitality'.

The Royal Netherlands Army and the personnel of Kamp Holterhoek in Eibergen, The Netherlands, for the use of their property and their practical assistance during the antenna height experiments. I would like to especially thank Henk Kloosterman and Norbert IJzereef, for their assistance in obtaining high speed direction finding data during two of our NVIS experiments.

Jens Mielich of the Leibniz Institute of Atmospheric Physics Kühlungsborn (Juliusruh ionosonde) for providing verified ionosonde data.

And so many others that did encourage and support me, in so many different ways. Sorry for not all mentioning you by name, but please know that your encouragement and help has been noted and very much appreciated. Thank you! 


\section{Publications of the author}

\section{Journal publications}

Near Vertical Incidence Skywave: Elevation Angles and Optimum Antenna Height for Horizontal Dipole Antennas, B. A. Witvliet, E. van Maanen, G. J. Petersen, A. J. Westenberg, M. J. Bentum, C. H. Slump, R. Schiphorst, IEEE Antennas and Propagation Magazine, Vol. 57, No. 1, February 2015.

Measuring the Isolation of Circularly Polarized Characteristic Waves in NVIS Propagation, B. A. Witvliet, E. van Maanen, G. J. Petersen, A. J. Westenberg, M. J. Bentum, C. H. Slump, R. Schiphorst, IEEE Antennas and Propagation Magazine, Vol. 57, No. 3, June 2015, pp. 120-130, 145.

\section{Conference publications}

Airborne Evaluation/Verification of Antenna Patterns of Broadcasting Stations, B. A. Witvliet, International Symposium on Electro-Magnetic Compatibility, Wroclaw, Poland, June 2006.

Mixed-Path Trans-Horizon UHF Measurements for P.1546 Propagation Model Verification, B. A. Witvliet, P. W. Wijninga, E. van Maanen, B. Smith, M. J. Bentum, R. Schiphorst, C. H. Slump, Antennas and Propagation in Wireless Communications, Torino, Italy, October 2011.

Medium Usage Model for the Design of Dynamic Spectrum Management in ISM Bands, B. A. Witvliet, M. J. Bentum, R. Schiphorst, C. H. Slump, International Conference on Communications, Ottawa, Canada, June 2012.

Dynamic Spectrum Management: Social Behavior for Radio Systems, B. A. Witvliet, M. J. Bentum, R. Schiphorst, C. H. Slump, CTIT Symposium The Innovation Highway, Enschede, The Netherlands, June 2012.

The Importance of Circular Polarization for Diversity Reception and MIMO in NVIS Propagation, B. A. Witvliet, E. van Maanen, G. J. Petersen, A. J. Westenberg, M. J. Bentum, C. H. Slump, R. Schiphorst, European Conference on Antennas and Propagation, The Hague, The Netherlands, April 2014.

Novel Method to Measure the Gain of UHF Directional Antennas using Distance Scan, B. A. Witvliet, E. van Maanen, M. J. Bentum, C. H. Slump, R. Schiphorst, European Conference on Antennas and Propagation, The Hague, The Netherlands, April 2014. 
The Importance of Circular Polarization for Diversity Reception and MIMO in NVIS Propagation, B. A. Witvliet, E. van Maanen, G. J. Petersen, A. J. Westenberg, M. J. Bentum, C. H. Slump, R. Schiphorst, Netherlands Antenna Research Framework, Enschede, The Netherlands, September 2014 [Best Poster award].

Near Vertical Incidence Skywave (NVIS) Antenna and Propagation Research in The Netherlands, B. A. Witvliet, E. van Maanen, G. J. Petersen, A. J. Westenberg, M. J. Bentum, C. H. Slump, R. Schiphorst, URSI Benelux Forum, Louvain, Belgium, November 2014 [Best Poster award].

Characteristic Wave Diversity in Near Vertical Incidence Skywave Propagation, B. A. Witvliet, E. van Maanen, G. J. Petersen, A. J. Westenberg, M. J. Bentum, C. H. Slump, R. Schiphorst, European Conference on Antennas and Propagation, Lissabon, Portugal, April 2015.

A Novel Method for the Evaluation of Polarization and Hemisphere Coverage of HF Radio Noise Measurement Antennas, B. A. Witvliet, E. van Maanen, M. J. Bentum, C. H. Slump, R. Schiphorst, IEEE International Symposium on Electromagnetic Compatibility, Dresden, Germany, August 2015. 


\section{Author Biography}

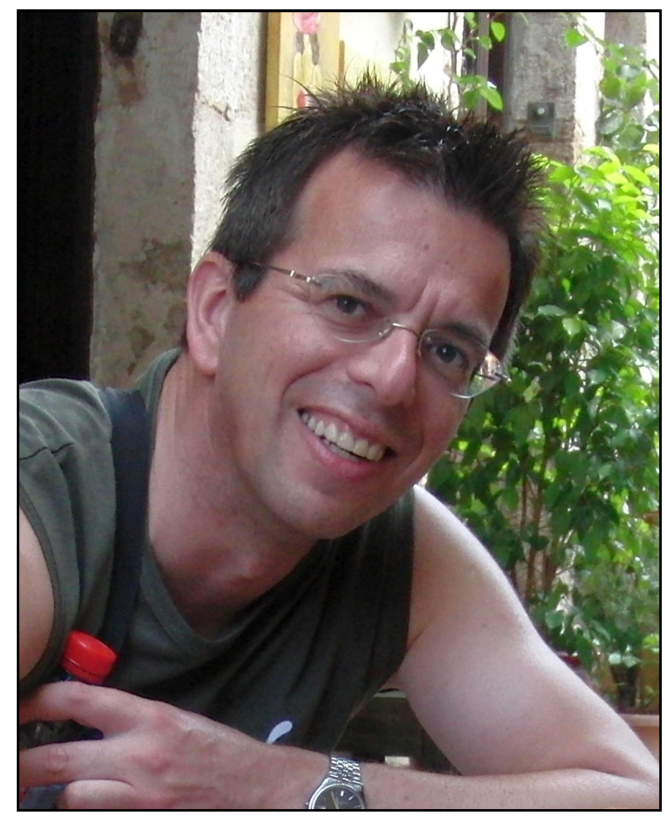

Ben A. Witvliet was born in Biak, Netherlands New Guinea in 1961. He received his BSc in Electronics and Telecommunications in 1988 in Enschede, The Netherlands.

He has working experience in electrical and electronic maintenance in Israel, in international telecommunication network management in The Netherlands, as Chief Engineer of the high power shortwave radio station of Radio Netherlands World Service in Madagascar and as manager of a team of technical specialists for TV, FM and AM broadcast transmitter operator NOZEMA in The Netherlands.

Since 1997 he works for Radiocommunications Agency Netherlands, currently as Technical Advisor. Research conducted for the Radiocommunications Agency includes long term long distance UHF propagation measurements, the design and realization of a helicopter-based system to measure antenna diagram and effective isotropically radiated power of FM broadcast stations, electromagnetic HF background noise measurement and UHF antenna gain measurements.

Since 2011 he combines his work with a part-time PhD research position in the Telecommunication Engineering group of the University of Twente, The Netherlands. His PhD research topic is Near Vertical Incidence Skywave antenna and propagation interaction and its application to emergency communications. He is a Senior Member of the IEEE and a member of European Association on Antennas and Propagation (EurAAP).

A more detailed and regularly updated author profile can be found on:

http://www.researchgate.net/profile/Ben_Witvliet/info

https://nl.linkedin.com/pub/ben-witvliet/7/97/536 


\section{- 180 -}


Cover photo: Flooded New Orleans following Hurricane Katrina, 11 September 2005.

Photo courtesy of National Oceanic and Atmospheric Administration (NOAA).

Commander Mark Moran, of the NOAA Aviation Weather Center, and Lt. Phil Eastman and Lt. Dave Demers, of the NOAA Aircraft Operations Center, all commissioned officers of the NOAA Corps, flew more than 100 hours surveying Katrina's devastation. Eastman piloted NOAA's Bell 212 Twin Huey Helicopter from August 31 to September 19. All three men took dozens of aerial photos from an altitude of several feet to 500 feet.

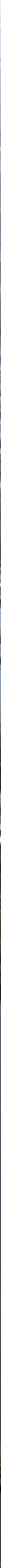

